\title{
ESTUDIO AERODINÁMICO EXPERIMENTAL EN FLUJO TURBULENTO DE BAJO REYNOLDS SOBRE ALAS CON MOVIMIENTO DE CABECEO
}

\author{
Tesis de Doctorado \\ SANTIAGO ALGOZINO
}

Presentada ante la Facultad de Ingeniería de la

Universidad Nacional de La Plata

como requisito para la obtención del grado académico de

\section{DOCTOR EN INGENIERÍA}

Dirección de Tesis:

Director: Dr. Ing. Delnero, Juan Sebastián

Co-Director: Dr. Ing. Marañón Di Leo, Julio

Jurado de Tesis:

Dr. Ing. Elaskar, Sergio Amado

Dr. Ing. Sacco, Carlos

Ing. García, Jorge Osvaldo

La Plata, 10 de octubre 2019 



\section{Resumen}

Desde los principios de la historia de la aviación, el hombre intenta imitar a las aves para poder volar. En las últimas décadas, con el diseño y avances en micro vehículos aéreos, producto de sus bajas velocidades de desplazamiento, se investiga la aplicación de sistemas de alas batientes. En este marco, se vienen desarrollando distintas investigaciones para poder comprender la aerodinámica de estos sistemas, dividiendo los movimientos en tres movimientos principales (aleteo, cabeceo y deslizamiento). En particular, el movimiento de cabeceo es de gran estudio por su relación con el fenómeno de pérdida dinámica, el cual está presente principalmente en palas de helicópteros y de aerogeneradores, el cual produce un retraso en la entrada en pérdida, y un incremento en la fuerza de sustentación generada, respecto a un incremento cuasi-estático en el ángulo de ataque. Si bien este efecto es conocido, es muy escaza la información respecto al efecto de la turbulencia en estas condiciones no estacionarias. Bajo estas condiciones se plantearon ensayos experimentales en túnel de viento de dos modelos alares (placa plana y perfil SD8020), para la medición de carga aerodinámica y el campo de presiones en la estela del modelo alar.

En primera instancia se realizaron mediciones con anemometría de hilo caliente para la determinación de las características de la corriente libre en el túnel de viento, y mediante la aplicación de una grilla turbuladora se modificaron las características turbulentas, generando un aumento en la intensidad de turbulencia y un decrecimiento de la escala integral espacial.

Del análisis de resultados, se pudieron encontrar diferencias en la carga aerodinámica desarrollada por cada modelo alar en función de las características de la corriente libre, tanto para condiciones estáticas como con movimiento de cabeceo a distintas frecuencias reducidas entre 0,015 y 0,06 . La influencia de la turbulencia en las cargas aerodinámicas es altamente dependiente de la geometría del modelo ensayado, principalmente de su borde de ataque y borde de fuga, en concordancia con resultados de otros autores para condiciones de flujo laminar en condiciones estáticas. La influencia de la turbulencia en las cargas aerodinámicas muestra un comportamiento similar en condiciones estáticas como dinámicas, generando modificaciones del 10\% o más en el coeficiente de sustentación.

A su vez, se realizaron mediciones en la estela con rack de presiones, para los casos estudiados. Los resultados muestran un efecto de la turbulencia en las estructuras vorticosas desprendidas por el modelo alar en condiciones dinámicas. Específicamente, el aumento de la intensidad de turbulencia produce una estela más homogénea, con estructuras coherentes de menor intensidad energética, y nuevamente, estos efectos son dependientes de la geometría del perfil utilizado. 
«Eppur si vola» ${ }^{1}$

“Le Vol des Insectes”, Magnar, 1934.

${ }^{1}$ Frase que hace referencia a que las leyes de la aerodinámica clásica no pueden explicar el vuelo de los insectos. 


\section{Agradecimientos}

Este trabajo de tesis ha sido posible gracias a la ayuda de muchas personas a lo largo de los distintos años de trabajo, las cuales me brindaron tiempo, dedicación $\mathrm{y}$, sobre todo, apoyo emocional. Es por ello que se merecen una mención especial.

En primera instancia, agradecer a mis directores, por su continuo apoyo y confianza, para mejorar día a día, e ir sorteando los distintos obstáculos que se fueron presentando en cada medición, ensayo y horas de análisis de datos. Su continuo apoyo y confianza fueron indispensables para poder llegar a cumplir con el objetivo planteado. Gracias Seba y Julio, por tanto conocimiento compartido y darme todas las herramientas para realizar los estudios.

A todos mis compañeros del UIDET-LaCLyFA, quienes estuvieron codo a codo ayudando en los ensayos, dando ese aliento para mejorar los ensayos y hasta dedicando horas colaborando en cada medición y puesta a punto. Muy especialmente, a Leo y Javier, que gracias a sus trabajos muchos ensayos fueron posibles, fueron indispensables en los ensayos. A Mariano, por todas esas charlas de oficina, de discusiones sobre distintos resultados y tantas horas compartidas que fortalecían los resultados obtenidos. A Nacho, por su continuo interés y ayudas con los detalles mecánicos y de movimiento. Infinitas gracias a todos ustedes.

A mi familia, especialmente a Bel, quienes estuvieron dando todo ese apoyo emocional necesario cuando las cosas no funcionaban, dando ese aliento necesario, esa palmada en la espalda o ese abrazo muchas veces necesario. Sin todo ese sostén, no sé si hubiera llegado hasta donde se llegó.

A la Facultad de Ingeniería, mi segunda casa durante tantos años, por darme todas las herramientas necesarias, y brindar todo el conocimiento por 12 años.

Al CONICET, por brindarme el apoyo económico necesario para realizar los estudios.

$\mathrm{Y}$ a todos los que directa, o indirectamente, han colaborado para poder realizar esta tesis doctoral.

Eternamente agradecido a todos ustedes. 


\section{Índice}

RESUMEN

LISTA DE SÍMBOLOS Y ABREVIATURAS …......................................................

CAPÍTULO 1: ANTECEDENTES.............................................................................1

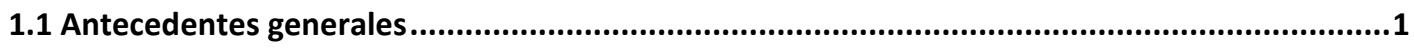

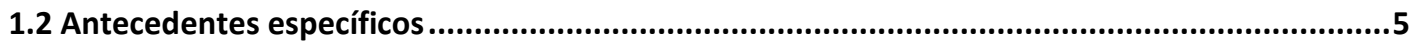

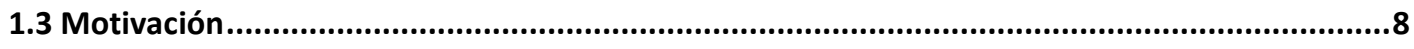

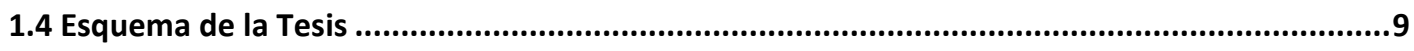

CAPÍTULO 2: METODOLOGÍA DE ENSAYO ………………................................11

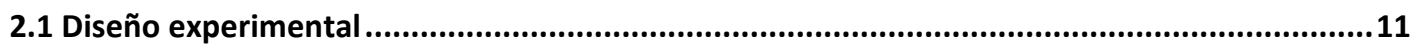

2.2 Caracterización de la corriente libre......................................................................................13

2.3 Mediciones de fuerza .........................................................................................................15

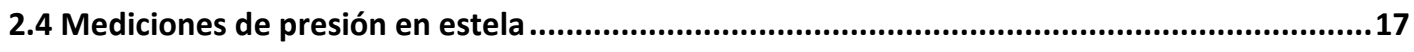

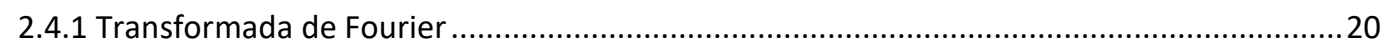

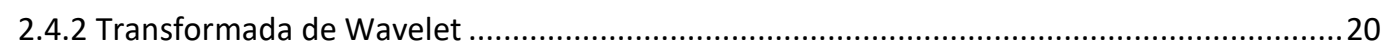

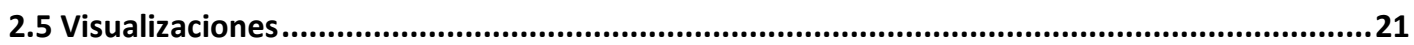

CAPÍTULO 3: RESULTADOS Y ANÁLISIS........................................................ 22

3.1 Caracterización del Túnel de Viento ..................................................................................22

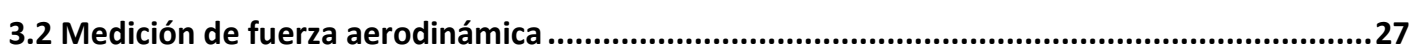

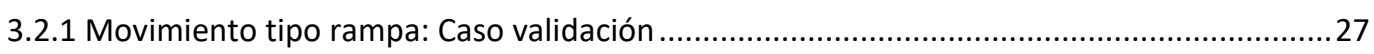

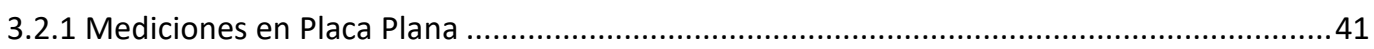

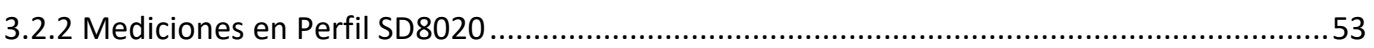

3.3 Presiones en la estela del modelo alar ..............................................................................62

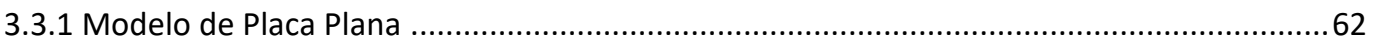

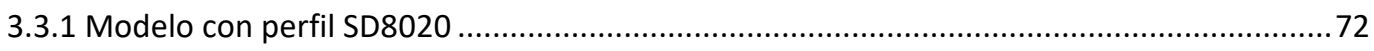

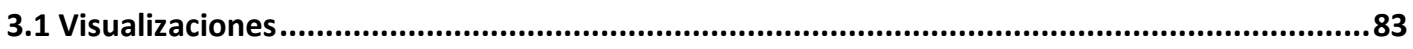

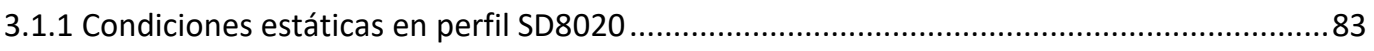

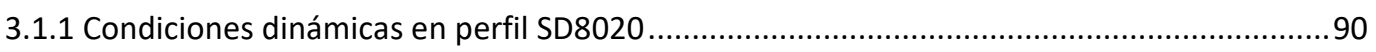

CAPÍTULO 4: CONCLUSIONES Y DISCUSIÓN ................................................... 93 


\section{Índice de Tablas}

Tabla 3.1. Ángulo medio del vector velocidad en la sección de prueba para las distintas configuraciones

Tabla 3.2 Coeficientes aerodinámicos para ángulo de ataque $45^{\circ}$ con mínima turbulencia..............43

Tabla 3.3. Coeficientes aerodinámicos para ángulo de ataque $45^{\circ}$ con intensidad de turbulencia de $1 \%$.

Tabla 3.4. Valores de sustentación para Reynolds 23.000, estático y dinámico para placa plana....53

Tabla 3.5. Cociente entre histéresis $\left(\Delta C_{1}\right)$ y el coeficiente máximo de sustentación. ........................53

Tabla 3.6. Coeficiente de sustentación máximo en función del Reynolds y la intensidad de turbulencia.

Tabla 3.7. Valores de sustentación para Reynolds 23.000, estático y dinámico para perfil SD8020. 62

Tabla 3.8. Cociente entre histéresis $\left(\Delta C_{l}\right)$ y el coeficiente máximo de sustentación. .62

\section{Índice de Figuras}

Figura 1.1. DelFly I, II y micro. Imagen extraída de Marino, 2013.

Figura 1.2. MAV desarrollado por la Universidad de Tamkang. Imagen extraída de Lin et al. 2010...1

Figura 1.3. Descripción de los ángulos de movimiento. Imagen extraída de Shy et al. 2010................3

Figura 1.4. Fenómeno de aplauso y separación. Imagen extraída de Shy et al. 2010 ..........................3

Figura 2.1. Pórtico y túnel de viento en la izquierda. Detalle del mecanismo de movimiento en la derecha

Figura 2.2. Acoples con balanza aerodinámica........................................................................... 11

Figura 2.3. Evolución del ángulo de ataque para distintas iteraciones, en función del tiempo adimensionalizado con el periodo del movimiento, verificando la repetitividad del movimiento .............12

Figura 2.4. Modelos Alares utilizados. En la izquierda placa plana, en la derecha perfil SD8020 .....13

Figura 2.5. Instrumental utilizado en la izquierda. Montaje en la sección de prueba del sensor anemométrico. 14

Figura 2.6. Grilla generadora de turbulencia localizada luego de la contracción del túnel de viento.

Figura 2.7. Montaje en túnel de viento del ala con su sistema de rotación.

Figura 2.8.Evolución del ángulo de ataque en el tiempo adimensionalizado con el periodo, en el

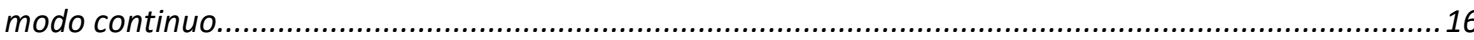

Figura 2.9. Señal sin procesamiento de filtrado, y filtrada para eliminar vibraciones mecánicas. ....16

Figura 2.10. Elemento equivalente montado en el túnel de ensayo ...............................................17

Figura 2.11. Rack de presiones ubicado en la sección del túnel de viento ......................................... 18

Figura 2.12. Estaciones de medición de estela.................................................................................. 18

Figura 2.13. Esquema con medidas del rack de presiones. Las medidas no mostradas corresponden a una medida idéntica a la inferior más cercana. Todas las dimensiones son en milímetros. ..................19

Figura 2.14. Esquema del montaje del arreglo óptico para generación de haz de luz.......................21

Figura 2.15. Demostración de visualización de flujo mediante humo y plano de luz láser ................21

Figura 3.1. Velocidad longitudinal media para cada grilla de turbulencia adimensionalizada con la velocidad de referencia. "Grilla 0 " indica sin grilla, "Grilla 15" indica grilla con apertura de $15 \mathrm{~mm}$........22

Figura 3.2. Velocidad media vertical para cada grilla en las tres velocidades utilizadas...................23 
Figura 3.3. Escala integral espacial en la componente longitudinal. Usando criterio 1/e en la auto correlación.

Figura 3.4. Escala espacial integral en la componente vertical de la velocidad. Usando criterio $1 / e$ en la auto correlación.

Figura 3.5. Intensidad de Turbulencia de la componente de velocidad longitudinal. ........................ 25

Figura 3.6. Intensidad de turbulencia de la componente de velocidad vertical. ..................................25

Figura 3.7. Espectro de energía de la componente longitudinal para las tres velocidades de referencia......

Figura 3.8. Espectro de energía de la componente vertical de la velocidad para las tres velocidades de referencia.

Figura 3.9. Distintas frecuencias reducidas analizadas para la condición de Reynolds 23000 con movimiento tipo rampa hasta $45^{\circ}$ de ángulo de ataque.

Figura 3.10. Coeficiente de sustentación teórico para tres valores diferentes de frecuencia reducida al mismo número de Reynolds (23000).

Figura 3.11. Comparación entre cinemática realizada por el ala y la enviada ..................................31

Figura 3.12. Respuesta de los filtros digitales implementados ..................................................... 31

Figura 3.13. Media experimental (azul), estimación teórica (negro punteado) y valor cuasi estático

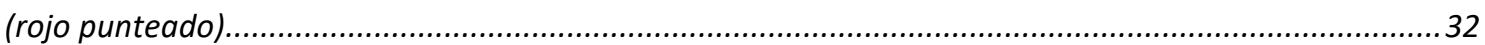

Figura 3.14. Tipos de movimientos ensayados en la referencia Ol y Babinsky 2016 ........................33

Figura 3.15. Resultados expuestos por M. Ol y H. Babinsky 2016 para un movimiento puro de cabeceo

Figura 3.16. Efecto del punto de pívot. Referencia Ol y Babinsky 2016. ........................................34

Figura 3.17. Comparación de resultados dinámicos con referencia bibliográfica. Izquierda movimiento que dura 6 tiempos convectivos (s/c) (Ol y Babinsky 2016), derecha movimiento que dura 16 tiempos convectivos.

Figura 3.18. Media de los casos dinámicos a distintos números de Reynolds y distintas frecuencias reducidas.

Figura 3.19. Comparación de resultados entre distintas turbulencias para distintos números de Reynolds y frecuencias reducidas.

Figura 3.20. Visualización con humo para distintos ángulos de ataque en estacionario. .................40

Figura 3.21. Secuencia temporal para ángulo de ataque de $10^{\circ}$................................................4 41

Figura 3.22. Secuencia de imágenes del caso dinámico con frecuencia reducida de 0.025 para un Re de 23000. La secuencia comienza en la esquina superior izquierda y evoluciona temporalmente con un paso temporal de 0,033 segundos siguiendo una disposición vertical.

Figura 3.23. Curva $C L$ y Cd versus ángulo de ataque para distintos Reynolds con mínima intensidad de turbulencia.

Figura 3.24. Eficiencia aerodinámica en función del ángulo de ataque y del número de Reynolds. Mínima intensidad de turbulencia

Figura 3.25. Pendiente de la curva de sustentación en función del ángulo de ataque. Mínima intensidad de turbulencia.

Figura 3.26. Coeficiente de sustentación y Resistencia para distintos movimientos y ángulos de ataque. Grandlund et al. 2011. Re: 20000. Flujo Laminar.

Figura 3.27. Cl y CD para distintos perfiles. Re: 10000. McArthur, 2016. Flujo Laminar................... 45

Figura 3.28. Coeficientes aerodinámicos para distintos números de Reynolds con alta turbulencia. 46

Figura 3.29. Eficiencia aerodinámica para los distintos números de Reynolds con alta turbulencia. 46

Figura 3.30. Polar de coeficientes aerodinámicos para distintos Reynolds con alta turbulencia. .....47

Figura 3.31.Comparación de coeficientes aerodinámicos para distintas turbulencias. Las barras indican 1 STD 48

Figura 3.32. Evolución de la carga aerodinámica en el tiempo adimensional ..................................49 
Figura 3.33. Coeficiente de sustentación en función del ángulo de ataque para distintas frecuencias reducidas y número de Reynolds. ...................................................................................................50

Figura 3.34. Evolución de las cargas aerodinámicas en función del tiempo adimensional...............51

Figura 3.35. Cargas aerodinámicas en función del ángulo de ataque ............................................51

Figura 3.36. Comparación de las cargas aerodinámicas para distintos niveles de intensidad de

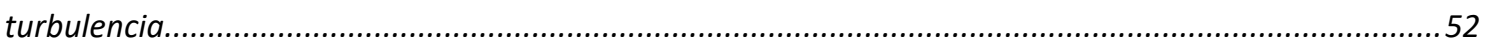

Figura 3.37. Distribución de presiones en perfil SD8020 para Reynolds $23000, \alpha=5^{\circ}$ e I $=0.5 \%$......55

Figura 3.38. Distribución de presiones en perfil SD8020 para Reynolds $23000, \alpha=5^{\circ}$ e $I=1 \%$.........55

Figura 3.39. Pendiente de la curva sustentación $\alpha$ para cada ángulo de ataque.............................55

Figura 3.40. Comparación de influencia del Reynolds para cada turbulencia incidente...................56

Figura 3.41. Comparación de resultados experimentales con simulaciones en XFoil, para los tres números de Reynolds ensayados y las dos condiciones de turbulencia incidente.

Figura 3.42. Comparación del coeficiente de sustentación a distintas intensidades de turbulencia para los tres números de Reynolds analizados.

Figura 3.43. Cargas en función del ángulo de ataque para las distintas iteraciones realizadas. Caso de mínima turbulencia, Reynolds 23000 y máxima frecuencia reducida. ..............................................58

Figura 3.44. Comparación de las cargas en tiempo adimensional para los 11 ciclos en una iteración particular. Reynolds 23000 y $k=0,06$.

Figura 3.45. Comparación de las distintas frecuencias reducidas en tiempo adimensional para ambos niveles de turbulencia. $\operatorname{Re}=23000$

Figura 3.46. Comparación entre distintas frecuencias reducidas en A para ambas intensidades de turbulencia.

Figura 3.47. Comparación entre cargas en caso dinámica y estático versus $\alpha$ para ambas intensidades de turbulencia. Re: 23000.

Figura 3.48. Comparación entre cargas en caso dinámica y estático versus $\alpha$ para ambas intensidades de turbulencia. Re: 57000.

Figura 3.49. Coeficiente de Presiones en función de la distancia al eje de rotación. Ángulo de ataque fijo en $3^{\circ}$ y velocidad de corriente libre de $8 \mathrm{~m} / \mathrm{s}$. Las barras representan un desvío estándar.

Figura 3.50. Correlación cruzada entre el sensor localizado a la altura del eje de rotación y los demás sensores, para el caso de Reynolds 23.000, $k=0,015$ y ambas intensidades de turbulencia. Estación 0,5C.

Figura 3.51. Correlación cruzada entre el sensor localizado a la altura del eje de rotación y los demás sensores, para el caso de Reynolds 23.000, $k=0,03$ y ambas intensidades de turbulencia. Estación $0,5 \mathrm{C}$. .

Figura 3.52. Correlación cruzada entre el sensor localizado a la altura del eje de rotación y los demás sensores, para el caso de Reynolds 23.000, $k=0,06$ y ambas intensidades de turbulencia. Estación $0,5 \mathrm{C}$.

Figura 3.53. Correlación cruzada entre el sensor localizado a la altura del eje de rotación y los demás sensores, para el caso de Reynolds 46.000, $k=0,015$ y ambas intensidades de turbulencia. Estación 0,5C.

Figura 3.54. Correlación cruzada entre el sensor localizado a la altura del eje de rotación y los demás sensores, para el caso de Reynolds 46.000, $k=0,03$ y ambas intensidades de turbulencia. Estación $0,5 \mathrm{C}$. .

Figura 3.55. Correlación cruzada entre el sensor localizado a la altura del eje de rotación y los demás sensores, para el caso de Reynolds 57.000, $k=0,015$ y ambas intensidades de turbulencia. Estación 0,5C.

Figura 3.56. Correlación cruzada entre el sensor localizado a la altura del eje de rotación y los demás sensores, para el caso de Reynolds 23.000, $k=0,015$ y ambas intensidades de turbulencia. Estación 1C. 
Figura 3.57. Correlación cruzada entre el sensor localizado a la altura del eje de rotación y los demás sensores, para el caso de Reynolds 23.000, $k=0,03$ y ambas intensidades de turbulencia. Estación $1 C$.

Figura 3.58. Correlación cruzada entre el sensor localizado a la altura del eje de rotación y los demás sensores, para el caso de Reynolds $23.000, k=0,06$ y ambas intensidades de turbulencia. Estación $1 C$.

Figura 3.59. Correlación cruzada entre el sensor localizado a la altura del eje de rotación y los demás sensores, para el caso de Reynolds 46.000, $k=0,015$ y ambas intensidades de turbulencia. Estación 1C.

Figura 3.60. Correlación cruzada entre el sensor localizado a la altura del eje de rotación y los demás sensores, para el caso de Reynolds 46.000, $k=0,03$ y ambas intensidades de turbulencia. Estación 1C.

Figura 3.61. Correlación cruzada entre el sensor localizado a la altura del eje de rotación y los demás sensores, para el caso de Reynolds 57.000, $k=0,015$ y ambas intensidades de turbulencia. Estación 1C.

Figura 3.62. Correlación cruzada entre el sensor localizado a la altura del eje de rotación de la estación 0,5C y los sensores de la estación 1C, para el caso de Reynolds $23.000, k=0,015$ y ambas intensidades de turbulencia.

Figura 3.63. Correlación cruzada entre el sensor localizado a la altura del eje de rotación de la estación 0,5C y los sensores de la estación 1C, para el caso de Reynolds 23.000, $k=0,03$ y ambas intensidades de turbulencia.

Figura 3.64. Correlación cruzada entre el sensor localizado a la altura del eje de rotación de la estación 0,5C y los sensores de la estación 1C, para el caso de Reynolds $23.000, k=0,06$ y ambas intensidades de turbulencia.

Figura 3.65. Correlación cruzada entre el sensor localizado a la altura del eje de rotación de la estación 0,5C y los sensores de la estación 1C, para el caso de Reynolds $46.000, k=0,015$ y ambas intensidades de turbulencia.

Figura 3.66. Correlación cruzada entre el sensor localizado a la altura del eje de rotación de la estación 0,5C y los sensores de la estación 1C, para el caso de Reynolds $46.000, k=0,03$ y ambas intensidades de turbulencia.

Figura 3.67. Correlación cruzada entre el sensor localizado a la altura del eje de rotación de la estación 0,5C y los sensores de la estación 1C, para el caso de Reynolds $57.000, k=0,015$ y ambas intensidades de turbulencia.

Figura 3.68. Análisis de la aparición temporal de los vórtices para la condición de Reynolds 23.000 con mínima turbulencia en la estación 0,5C y $k=0,015$.

Figura 3.69. Mapa de Wavelets y Densidad de potencia. Reynolds $23.000, k=0,015$ para ubicación media cuerda aguas abajo del borde de fuga y ambas intensidades de turbulencia.

Figura 3.70. Mapa de Wavelets y Densidad de potencia. Reynolds 23.000, $k=0,03$ para ubicación media cuerda aguas abajo del borde de fuga y ambas intensidades de turbulencia.

Figura 3.71. Mapa de Wavelets y Densidad de potencia. Reynolds 23.000, $k=0,06$ para ubicación media cuerda aguas abajo del borde de fuga y ambas intensidades de turbulencia.

Figura 3.72. Mapa de Wavelets y Densidad de potencia. Reynolds 23.000, $k=0,015$ para ubicación una cuerda aguas abajo del borde de fuga y ambas intensidades de turbulencia.....

Figura 3.73. Mapa de Wavelets y Densidad de potencia. Reynolds 23.000, $k=0,03$ para ubicación una cuerda aguas abajo del borde de fuga y ambas intensidades de turbulencia.....

Figura 3.74. Mapa de Wavelets y Densidad de potencia. Reynolds 23.000, $k=0,06$ para ubicación una cuerda aguas abajo del borde de fuga y ambas intensidades de turbulencia....................................78

Figura 3.75. Mapa de Wavelets de distintos sensores, para el caso de Reynolds $23.000, k=0,06$, mínima turbulencia y el rack alejado media cuerda de distancia. 
Figura 3.76. Mapa de Wavelets de distintos sensores, para el caso de Reynolds $23.000, k=0,06$, máxima intensidad de turbulencia y el rack alejado media cuerda de distancia.

Figura 3.77. Correlación cruzada entre tomas de una estación, respecto al sensor localizado en el eje de rotación para distintas frecuencias reducidas e intensidad de turbulencia. Re: 23000, estación a media cuerda aguas abajo.

Figura 3.78. Correlación cruzada entre tomas de una estación, respecto al sensor localizado en el eje de rotación para distintas frecuencias reducidas e intensidad de turbulencia. Re: 23000, estación a una cuerda aguas abajo.

Figura 3.79. Correlación cruzada entre la toma ubicada en el eje de rotación de la estación 0,5C con todas las tomas de presión de la estación 1C. Reynolds 23000 y distintas frecuencias reducidas e intensidades de turbulencia.

Figura 3.80. Mapa de Wavelets y Densidad de potencia. Reynolds 23.000, $k=0,015$ para ubicación media cuerda aguas abajo del borde de fuga y ambas intensidades de turbulencia. Modelo Alar: Perfil SD8020

Figura 3.81. Mapa de Wavelets y Densidad de potencia. Reynolds 23.000, $k=0,03$ para ubicación media cuerda aguas abajo del borde de fuga y ambas intensidades de turbulencia. Modelo Alar: Perfil SD8020

Figura 3.82. Mapa de Wavelets y Densidad de potencia. Reynolds 23.000, $k=0,06$ para ubicación media cuerda aguas abajo del borde de fuga y ambas intensidades de turbulencia. Modelo Alar: Perfil SD8020

Figura 3.83. Mapa de Wavelets y Densidad de potencia. Reynolds $46.000, k=0,015$ para ubicación media cuerda aguas abajo del borde de fuga y ambas intensidades de turbulencia. Modelo Alar: Perfil SD8020

Figura 3.84. Mapa de Wavelets y Densidad de potencia. Reynolds $46.000, k=0,03$ para ubicación media cuerda aguas abajo del borde de fuga y ambas intensidades de turbulencia. Modelo Alar: Perfil SD8020

Figura 3.85. Mapa de Wavelets y Densidad de potencia. Reynolds 57.000, $k=0,015$ para ubicación media cuerda aguas abajo del borde de fuga y ambas intensidades de turbulencia. Modelo Alar: Perfil SD8020

Figura 3.86. Visualización con humo para condición estática del ángulo de ataque........................91

Figura 3.87. Secuencia de imágenes para caso dinámico con frecuencia reducida $k=0.025$...........92 


\section{Lista de Símbolos y Abreviaturas}

$\alpha$ : ángulo de ataque, en ${ }^{\circ}$

$v$ : Viscosidad cinemática del fluido, $1,74 \times 10^{-5}$

c: cuerda del modelo, en m

Cl: Coeficiente de sustentación, adimensionalizado con superficie alar y presión dinámica.

Cd: Coeficiente de resistencia

CP: Coeficiente de Presiones

f: frecuencia de oscilación, en $\mathrm{Hz}$

$\mathrm{k}$ : frecuencia reducida, $\pi f c /$ Uref

$\mathrm{K}$ : frecuencia reducida para caso no periódico, $\frac{\dot{\theta} c}{2 U r e f}$

LEV: Vórtice de borde de ataque (Leading Edge vortex en inglés).

MUAV o MAV: micro vehículo aéreo no tripulado, por sus siglas en inglés.

Re: Número de Reynolds, $\frac{U_{\infty} c}{v}$

S: Superficie de referencia, cuerda * envergadura

t/c: espesor adimensionalizado con la cuerda del ala.

TEV: vórtice de borde de fuga, (Trailing Edge vortex en inglés).

UAV: vehículo aéreo no tripulado, por sus siglas en inglés.

Uref o $U_{\infty}$ : Velocidad de la corriente libre, en $\mathrm{m} / \mathrm{s}$ 


\section{CAPÍTULO 1: Antecedentes}

\subsection{Antecedentes generales}

En la última década ha sido de gran interés el desarrollo en diseños de alas batientes ("flapping wings"), dado el continuo desarrollo de los micro vehículos aéreos (MAV - micro aerial vehicles - micro vehículo aéreos), ya que dichas aeronaves obtienen todas las ventajas de sistemas de vuelo mediante aleteo respecto a los sistemas convencionales de generación de sustentación de ala fija o las rotatorias de propulsión (hélices) (Muller, 2001). Un ejemplo de ello es la posibilidad de generar sustentación sin la necesidad de avanzar. En el aleteo, la generación de sustentación y empuje se producen de forma simultánea (Jadhav, 2007), y las inestabilidades aerodinámicas propias de este tipo de vuelos permiten una alta maniobrabilidad en ambientes desfavorables, necesitándose altos valores de eficiencia en empuje y propulsión.

Los MAV, desarrollados o en desarrollo, hoy en día provienen de la observación del vuelo de las aves en la naturaleza Brown, 1948 y Shyy et al., 1999 revelando que los pájaros no solo usan el clásico movimiento combinado de cabeceo y alabeo (o flapping) para el aleteo, sino que también incorporan un movimiento de deslizamiento (gliding) en dirección de la velocidad de la corriente libre incidente. Al incorporar esta mecánica para la generación de sustentación y empuje, se logra un gran potencial para que el vehículo pueda alcanzar zonas inhóspitas o de acceso complicado para el monitoreo ambiental o seguridad. Algunos ejemplos de estos desarrollos pueden ser los vehículos Delfly 1 ( $50 \mathrm{~cm}$ envergadura, $21 \mathrm{~g})$, Delfly 2 ( $28 \mathrm{~cm}$ envergadura, $16.07 \mathrm{~g}$ ) and Delfly micro (10 cm envergadura, $3.07 \mathrm{~g}$ ), desarrollado por TU Delft, los cuales cuentan con un sistema de video. Otro ejemplo puede ser el desarrollo realizado por la Universidad de Tamkang (Taiwán), el cual es el primer vehículo aéreo con menos de 10 gramos de peso en realizar un vuelo completamente autónomo.

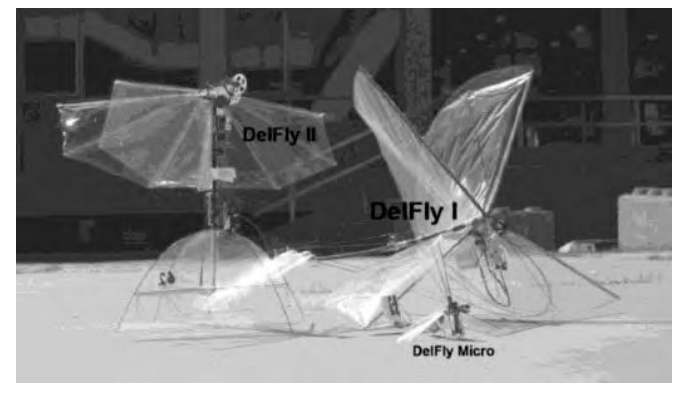

Figura 1.1. DelFly I, II y micro. Imagen extraída de Marino, 2013.

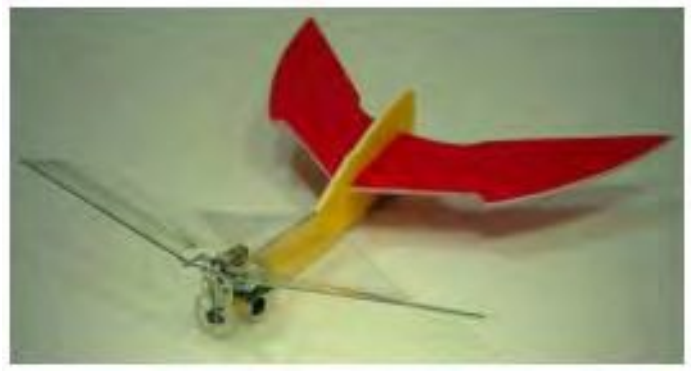

Figura 1.2. MAV desarrollado por la Universidad de Tamkang. Imagen extraída de Lin et al. 2010

La gran variedad en forma y cinemática que presentan las distintas aves e insectos en sus vuelos, generan un amplio espectro de estudio, sumado a las inestabilidades dinámicas propias de la aerodinámica, concluyen en un estudio muy complejo del tema. Es por esto que en general se realizan estudios primeramente de 
movimientos puros (flapping, cabeceo y gliding) para luego analizar los acoplamientos de los distintos movimientos según los ejes. Estos movimientos además generan efectos acoplados que influenciarán en distintas maneras según el desfasaje que tengan entre sí. Además, ya se conoce que la aerodinámica desarrollada durante el movimiento del ala está altamente relacionada con algunas magnitudes adimensionales de la fluidodinámica, la cinemática del ala y otros parámetros adimensionales normalmente utilizados. En los estudios de alas con movimiento, los más relevantes son:

i. Número de Reynolds (Re): Dicho número representa el cociente entre las fuerzas inerciales y las fuerzas viscosas. Su fórmula convencional es:

$$
R e=\frac{L_{r e f} U_{r e f}}{v}
$$

ii. Número de Strouhal (St): Esta relación describe la influencia relativa de la velocidad incidente $\left(\mathrm{U}_{\infty}\right)$ con respecto a la velocidad de rotación. Este número adimensional caracteriza la dinámica de los vórtices generador en la estela por el vuelo en flapping en avance.

$$
S t=\frac{f L_{r e f}}{U_{\infty}}
$$

iii. Frecuencia reducida (k): Este número representa de mejor modo las inestabilidades asociadas a las alas con batimiento que el número de Strouhal, por comparación de la distribución de las longitudes de onda espaciales a lo largo de la longitud de la cuerda. Está definido por la velocidad angular ( $2 \pi f)$, la cuerda media y una velocidad de referencia de la siguiente forma:

$$
k=\frac{\pi f c_{m}}{U_{r e f}}
$$

Se ha demostrado en distintos artículos científicos (Ellington 1997; Shyy et al. 2010) que la cinemática del vuelo de las aves está compuesta por el movimiento del cuerpo y de las alas. Si se considera al cuerpo como un elemento rígido, y las alas vinculadas a este, se puede describir el movimiento de las mismas mediante tres ángulos que relacionan el plano del ala con el de batido $(\phi)$ como se puede ver en la Figura 1.3:

i. El ángulo de batido $(\phi)$ que describe la posición del ala dentro del plano de batido.

ii. El ángulo de rotación del ala respecto a la envergadura propia descripto por el ángulo de ataque $(\alpha)$.

iii. El ángulo de rotación del ala perpendicular al plano de batido dado por la elevación o ángulo de desviación $(\theta)$.

Para poder estudiar el fenómeno de flapping, es necesario comprender la dinámica de los vórtices, la interacción vórtice-ala y además la interacción fluido-estructura que se torna sumamente importante cuando se consideran estructuras flexibles.

Shyy et al. 2010 compilaron de toda la literatura disponible hasta dicha fecha, la descripción de los fenómenos fluidodinámicos que forman parte del mecanismo de flapping, y concluyó que los más importantes son: 


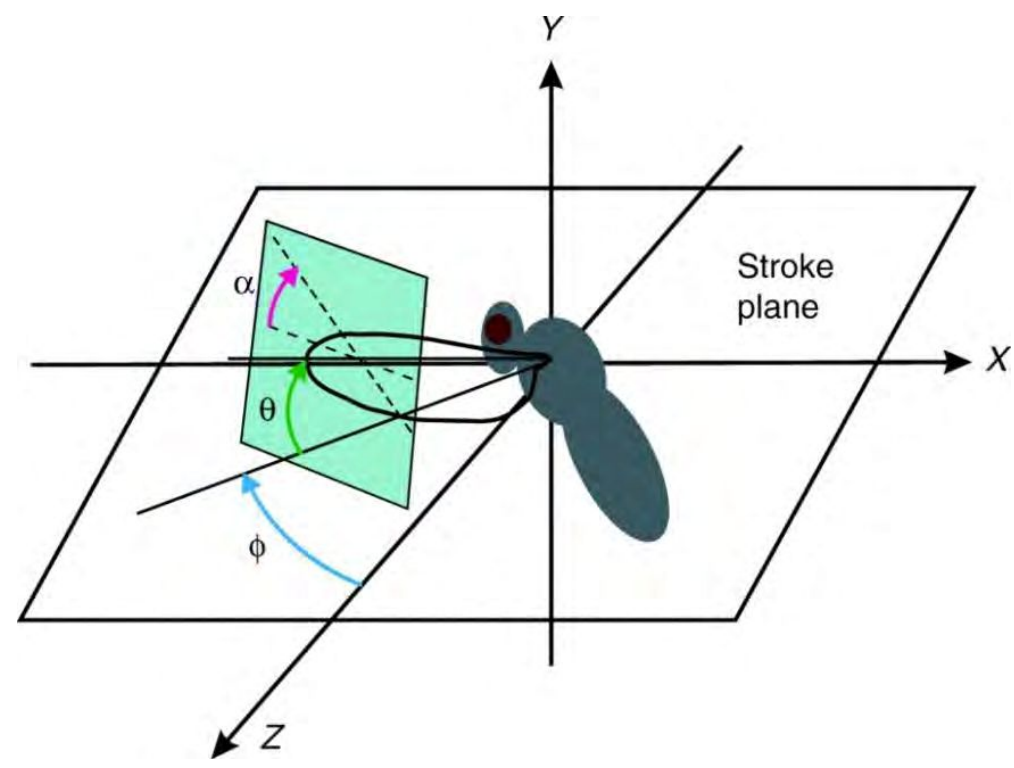

Figura 1.3. Descripción de los ángulos de movimiento. Imagen extraída de Shy et al. 2010

- Aplauso y Separación:

El primer mecanismo de generación de sustentación en régimen no estacionario para explicar cómo los insectos vuelan, fue el movimiento de aplauso y separación de una chalsidoidea (especie de avispa). Como no pudo explicar la generación extra de sustentación mediante la teoría de vuelo estacionario, él observó que la chalsidoidea junta sus dos alas una al lado de la otra y luego las separa sobre la línea horizontal de contacto para llenar de aire esa separación. Durante el periodo de separación el aire alrededor de cada ala adquiere circulación en la dirección correcta, de forma tal de generar sustentación extra. Un esquema de este procedimiento se muestra en la Figura 1.4. Luego, otros investigadores continuaron con la línea propuesta por Weis-Fogh, 1973 y realizaron estudios mediante simulaciones numéricas de este mecanismo y también mediciones mediante PIV (Particle Image Velocimetry) en mecanismos robóticos a escala que simulaban un movimiento similar al de este insecto, y ambos justificando el aumento en la sustentación debido al movimiento planteado en bajo número de Reynolds.

A

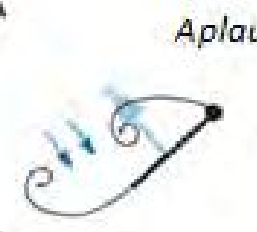

B

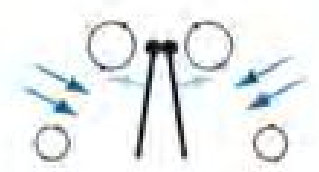

C

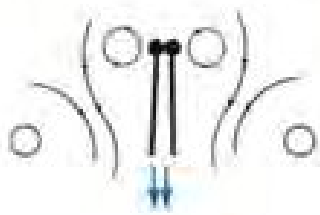

D

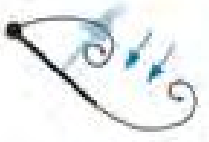

E

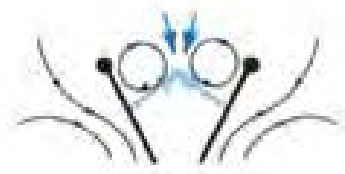

$\mathrm{F}$

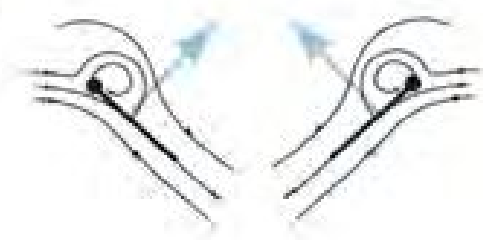

Figura 1.4. Fenómeno de aplauso y separación. Imagen extraída de Shy et al. 2010 


\section{“Antecedentes"}

- Rápida rotación de cabeceo:

Al finalizar cada aleteo, tanto en sentido ascendente como al finalizar el descendente, las alas pueden sufrir una rápida rotación de cabeceo, la cual incrementa la generación de fuerza aerodinámica. La diferencia entre el ángulo de cabeceo y el de traslación puede ser utilizada como parámetro control de la sustentación generada. Es decir, podemos tener rotación en avance (cabeceo antes de que termine la corrida ascendente, o descendente), o en atraso (cabeceo después de que termina la corrida).

- Captura de estela:

El mecanismo de captura de estela es también observado durante la interacción ala-estela. Cuando el ala cambia de sentido su traslación se encuentra con la estela generada por el batido previo, por lo que la velocidad efectiva del fluido aumenta, aumentando el pico de fuerza aerodinámica generada. Distintos investigadores examinaron este efecto de modelos simplificados de la mosca de la fruta usando robots dinámicamente escalados a un Reynolds de 1.0-2.0 x10². Ellos compararon la fuerza medida con la aproximación cuasi-estática y así obtener la influencia de la estela. Los resultados demuestran que la fuerza por la captura de estela representa un fenómeno completamente dinámico dependiente de cambios temporales en la distribución y magnitud de la vorticidad durante batimiento inverso.

- Entrada en pérdida retrasada del vórtice de borde de ataque (LEV):

Ellington y su grupo sugirieron que la entrada en pérdida retardada del vórtice de borde de ataque (LEV) puede promover la asociación de la generación de sustentación con el batimiento del ala. El LEV crea una zona de baja presión sobre el ala y por ende un aumento de la sustentación. Cuando el ala se va moviendo, el flujo se va separando desde el borde de ataque y de fuga, así como también en la punta del ala, y forma largos y organizados vórtices, conocidos como vórtice de borde de ataque (LEV), vórtice de borde de fuga (TEV) y vórtice de punta de ala (TiV), por sus siglas en inglés respectivamente. En alas batientes, la presencia de LEVs es esencial para retrasar la entrada en pérdida y para el aumento de producción de la fuerza aerodinámica durante la traslación del ala. Numerosos trabajos se han realizado, y se continúan investigando, para la comprensión de la estabilidad del LEV y sus efectos en las fuerzas aerodinámicas.

- Vórtice de punta de ala (TiV):

Está demostrado que los vórtices de punta de ala (TiVs) asociado a alas fijas reducen la sustentación e inducen resistencia. Pero, en flujos no estacionarios, los TiVs pueden influenciar la fuerza total que experimenta el ala en tres formas: (i) creando una zona de baja presión cerca de la punta del ala, (ii) mediante una interacción entre LEV y TiV, y (iii) creando una estructura en la raíz por un movimiento descendente y radial de los vórtices de raíz y los TiV. Para el movimiento de batimiento en vuelo estacionario, dependiendo de la cinemática específica, los TiV podrían promover o producir un pequeño impacto en la aerodinámica de un ala en flapping de baja relación de aspecto.

Dados estos fenómenos fluido dinámicos, es importante medir las capacidades de generación de sustentación y empuje que tiene el sistema, para poder analizar las ventajas o desventajas que produce cada uno. Es por ello que, mediante la utilización de balanzas aerodinámicas, para los ensayos experimentales, se miden principalmente estas dos fuerzas, obteniéndose los dos coeficientes adimensionales tradicionales:

- Coeficiente de sustentación $(\mathrm{Cl})$ : Este coeficiente adimensional nos determina la capacidad de generar sustentación que posee el sistema, tomando como referencia la velocidad y densidad de la corriente libre incidente $\left(\mathrm{U}_{\infty}, \mathrm{y} \rho\right.$ respectivamente), y el área de referencia del ala (normalmente, el área proyectada) para 


\section{"Estudio aerodinámico experimental en flujo turbulento de bajo Reynolds sobre alas con movimiento de cabeceo"}

poder comparar dicho coeficiente con los de otros sistemas. 1 es la fuerza de sustentación generada, dato a medir.

$$
C_{l}=\frac{2 l}{\rho U_{\infty}^{2} S}
$$

- Coeficiente de Empuje $\left(\mathrm{C}_{\mathrm{t}}\right)$ : En este caso, el aleteo genera de manera simultánea una fuerza de sustentación y otra de empuje (para contrarrestar la resistencia y poder avanzar), por lo cual es de interés medir dicha cantidad a partir de la medición del empuje (t), y ello se realiza con la siguiente fórmula:

$$
C_{t}=\frac{2 t}{\rho U_{\infty}^{2} S}
$$

\subsection{Antecedentes específicos}

Si bien el flujo desarrollado por las aves, dada la cinemática y los mecanismos de generación de fuerzas anteriormente citados, es altamente tridimensional, para su simplicidad se suelen estudiar por separado cada movimiento de rotación. Uno de los más estudiados es el movimiento de cabeceo, por su relación con el fenómeno de pérdida dinámica que ocurre en palas de rotores de helicópteros o en aerogeneradores. Este fenómeno involucra cambios bruscos en el ángulo de ataque (orientación de la velocidad incidente respecto al eje de la cuerda del perfil o ala) lo que produce desprendimientos y fenómenos no estacionarios sobre el flujo del perfil generando cambios en la distribución de presiones. Uno de los principales efectos que produce este fenómeno es el retraso de la entrada en pérdida, así como también un incremento en el coeficiente de sustentación y de momento de cabeceo (McCroskey 1982, Carr 1988). El problema principal radica en que, para todos estos estudios previos, el número de Reynolds, dada su aplicación a helicópteros, es dos órdenes de magnitud mayor a los aplicados a micro vehículos aéreos. Si el análisis se extiende a oscilaciones periódicas, se ha demostrado que al incrementar la frecuencia reducida se puede modificar el patrón de vórtices en la estela, generando empuje en vez de resistencia. A su vez, dicho movimiento es el principal generador de vórtices de borde de ataque y de fuga, los cuales generan un incremento en la sustentación total en el ciclo de oscilación, como fue detallado en el movimiento rápido de cabeceo mencionado en la sección anterior.

Uno de los principales efectos aerodinámicos que se producen en perfiles oscilatorios, es el retardo entre la respuesta aerodinámica y el movimiento, bien conocido para casos de dos grados de libertad, de cabeceo $(\alpha)$ y hundimiento (h). Uno de los modelos generales más simples desarrollados para poner evidencia este efecto es el modelo de Theodorsen (Leishman 2000), donde para movimientos armónicos de cabeceo y hundimiento, el coeficiente de sustentación en función del tiempo " $\mathrm{t}$ " toma la siguiente forma:

$$
\begin{aligned}
C_{l}(t)=2 \pi \alpha_{0}+ & \frac{\pi c}{2}\left(\frac{\dot{\alpha}}{U_{\infty}}+\frac{\ddot{h}}{U_{\infty}^{2}}-\frac{c\left(X_{p}-1\right) \ddot{\alpha}}{2 U_{\infty}^{2}}\right) \\
& +2 \pi\left(\frac{\dot{h}}{U_{\infty}}+\alpha-\alpha_{0}+\frac{c\left(1.5-2 X_{p}\right) \dot{\alpha}}{2 U_{\infty}}\right) C(k)
\end{aligned}
$$

Donde el primer término representa el término de fuerza estático, el segundo término es conocido como "masa aparente" o "masa agregada" o fuerza no circulatoria, debido a los efectos de aceleración. El tercer término modeliza los efectos circulatorios del perfil, donde $\mathrm{C}(\mathrm{k})$ es la Función compleja de Theodorsen con módulo menor a 1, la cual representa la atenuación en la amplitud de sustentación y retardo en la respuesta de la sustentación. Este modelo asume una estela plana, donde se cumple la condición de Kutta en el borde de fuga (el punto de remanso posterior coincide con el borde de fuga), por lo que excluye en su análisis la calle de vórtices que se forman, la convección de grandes cantidades de flujo separado, los vórtices desprendidos desde 


\section{“Antecedentes"}

borde de ataque y fuga. El punto interesante desde el punto de vista ingenieril, es su simplicidad para modelizar el fenómeno, por lo que si las amplitudes no son muy elevadas, puede ser utilizado con cierta confianza en los resultados obtenidos; $\mathrm{Ol}$ et al. 2010 realizó comparaciones de los esfuerzos medidos de forma experimental para un perfil SD7003 con una frecuencia reducida $\mathrm{k}=0,25 \mathrm{y}$ Reynolds 60.000 , encontrando muy buenas predicciones realizadas por la expresión de Theodorsen, aunque las condiciones del flujo eran sumamente no estacionarias y con grandes desprendimientos, por lo que las hipótesis que aplica el método analítico no se cumplían en el ensayo.

En los últimos años, un estudio de esfuerzos mundiales ha definido ciertos movimientos canónicos para el estudio del fenómeno de cabeceo, hundimiento y deslizamiento, producto del creciente interés en el tema (AVT202, 2016). En dicho estudio, se realizaron mediciones experimentales, simulaciones numéricas y se propuso una técnica analítica para la determinación de la carga aerodinámica ante este movimiento, llegando a un buen acuerdo entre las distintas instalaciones utilizadas, que comprenden túneles de viento, túneles de agua, tanques de agua, en lo que respecta a facilidades experimentales. Dicho análisis sirve como puntapié inicial para la comprensión del fenómeno fluido dinámico del movimiento de perfiles, pensando en la aplicación de micro vehículos aéreos, ante condiciones de flujo incidente laminar.

En AVT202, 2016, se analizaron diferentes cinemáticas (de traslación y rotación), que comprenden hundimiento y cabeceo. En todos los casos, los movimientos de cabeceo generaron mayores valores de sustentación que en los casos de hundimiento, producto del efecto de aceleración del ángulo de cabeceo. En este caso, los movimientos analizados son siempre del tipo rampa ascendente, con lo cual se analizó la respuesta luego de la finalización del movimiento, observándose que en este periodo estacionario del movimiento se produce una caída abrupta del coeficiente de sustentación seguido de un proceso de relajación a la condición estacionaria. Para los casos de aceleraciones elevadas, el pico en el valor de sustentación ocurre, aproximadamente, cuando la aceleración es finalizada. Para los movimientos más lentos, el valor del pico se encuentra a tiempos más tempranos, antes de que el movimiento sea finalizado. En este trabajo, el valor final del ángulo de ataque es de $45^{\circ}$, con lo cual es una zona de grandes ángulos de ataque, y el ángulo final es mucho mayor al ángulo de pérdida estática. En estos movimientos más lentos, el valor del pico de sustentación está relacionado con el tiempo de formación, saturación y desprendimiento del vórtice de borde de ataque, mientras que, para los casos de rápida rotación, el vórtice de borde de ataque sigue creciendo y acumulando vorticidad durante el movimiento. Además, en muchos de los ensayos han aparecidos segundos picos de sustentación, que están asociados a vórtices secundarios de borde de ataque, pero que no han podido determinar las variables principales que producen dichas formaciones, más bien fueron asociadas a la relación de aspecto de los modelos ensayados, así como las particularidades de la instalación de cada ensayo. Un punto importante hallado en dicho estudio es la relación entre la coherencia de los vórtices de borde de ataque con la frecuencia reducida del movimiento, siendo más coherentes a medida que se aumenta la frecuencia. A su vez, lograron determinar que la velocidad de desplazamiento del vórtice de borde de ataque corresponde a un valor entre un tercio y la mitad de la velocidad de la corriente libre, mientras que el vórtice desprendido por el borde de fuga se desplaza a una velocidad muy cercana a la de la corriente libre. Si bien los resultados obtenidos a partir de simulaciones numéricas y de mediciones mediante técnicas de PIV no fueron muy concluyentes, debido a la dispersión de los resultados hallados, la tendencia del movimiento de estos vórtices parece seguir un patrón bien marcado, muy similar al determinado por Wagner en 1925. Especialmente para los casos de velocidad de rotación "lenta", los vórtices desprendidos son más difíciles de identificar, y se ha determinado que sus características son más tridimensionales que cuando se incrementa la velocidad de rotación. Además, se ha podido identificar, que para el caso del movimiento de cabeceo, los términos de masa aparente y efecto Magnus son más importantes que en el caso de movimientos en el sentido de la corriente libre, donde la historia de las fuerzas está más relacionada con la separación entre los vórtices de borde de ataque y de fuga. Otro punto interesante determinado en este trabajo es la poca dependencia de la historia de la fuerza de sustentación con el número de Reynolds, excepto 


\section{"Estudio aerodinámico experimental en flujo turbulento de bajo Reynolds sobre alas con movimiento de cabeceo"}

para casos de numero de Reynolds menores a 100 donde la formación de vórtices no se produce. Además, uno de los grupos participantes en el trabajo realizó comparaciones entre dos modelos, uno con borde de ataque romo, o ángulo agudo con respecto a una placa plana con borde de ataque redondeado, encontrando que la fuerza de sustentación no es muy dependiente de la geometría del borde de ataque. Este resultado parece poco intuitivo en función de lo conocido de aerodinámica estacionaria para condiciones estacionarias en bajos números de Reynolds, donde la geometría del borde de ataque, especialmente su radio, determina la formación y forma de una burbuja de recirculación en la cara a succión del perfil, modificando la fuerza de sustentación generada por el perfil. Ver Conceptos de Aerodinámica General.

Si bien hay muchos estudios sobre la fuerza y el campo de velocidades de un ala o perfil con distintos patrones de movimiento, es muy escasa la información sobre el efecto de la turbulencia en estas condiciones. Para condiciones estacionarias, se pueden encontrar distintos estudios que fueron realizados en perfiles y diferentes condiciones turbulentas; algunos modificaron independientemente la intensidad y la escala de esta turbulencia. Por ejemplo, Kindelspire, 1988 estudió el efecto de la turbulencia en la corriente libre en un perfil en Reynolds 500.000 con intensidades de turbulencia hasta el $4 \%$ y escalas que varían desde 5\% a $18 \%$ del tamaño de la cuerda del perfil. Él detectó que la zona de transición de la capa límite laminar a turbulenta comienza de forma temprana al incrementar los niveles de intensidad de turbulencia, además, las escalas con tamaño similar al espesor de capa límite genera un incremento en este espesor de capa límite medio del perfil mientras que para escalas mayores, no encontró diferencias. Yap et al. 2001, experimentó con dos perfiles simétricos a niveles de turbulencia de $2.4 \%$ y $5.4 \%$ para Reynolds entre 120.000 y 190.000 . Sus resultados muestran que el incremento de la intensidad de turbulencia retrasa la entrada en pérdida aumentando los coeficientes de sustentación y resistencia. Cao en 2010 investigó la performance aerodinámica de perfiles no simétricos a Reynolds similares a los propuestos en este trabajo, entre 55.000 y 100.000 , modificando de forma independiente las escalas y la intensidad de turbulencia. Los resultados muestran que la entrada en pérdida del perfil nuevamente es retrasada al incrementar la intensidad de turbulencia desde $4 \%$ a $9 \%$, sin embargo, al aumentar las escalas de la turbulencia, la entrada en pérdida se adelanta. Además, encontró que para que la capa límite turbulenta sea modificada, las escalas turbulentas deben ser del orden de la capa límite. Al aumentar los niveles de turbulencia, ha encontrado incrementos en el coeficiente máximo de sustentación, para valores mayores a los de pérdida de flujo laminar. Finalmente, Cao determinó que a medida que aumentan los niveles de intensidad de turbulencia, el efecto de la escala empieza a ser poco determinante en las cargas aerodinámicas, independientemente del tamaño de estas.

Al realizar una revisión de los efectos de la turbulencia en condiciones dinámicas, no son muchos los trabajos que se pueden hallar. Algunos antecedentes, principalmente aplicados a palas de aerogeneradores han sido publicados. Ghandi, en 2017, al realizar la comparación de los resultados con y sin turbulencia mediante simulaciones numéricas directa, generando la turbulencia colocando un cilindro aguas arriba con Strouhal 0,216, encontró que la tendencia general de los coeficientes es similar para la misma frecuencia reducida $(0,16$ y 0,25$)$, observando un retraso en la entrada en pérdida al aumentar la turbulencia. A su vez, la pendiente de la curva sustentación versus tiempo es menor mientras se incrementa el ángulo de ataque, siendo el pico de sustentación del caso sin perturbación ligeramente mayor que en el caso con el cilindro aguas arriba. Al analizar la curva de resistencia, esta tiene una tendencia similar para el caso sin perturbación, pero cuando está perturbada la magnitud es menor en todo el ciclo. Los resultados obtenidos muestran efectos importantes de la estela turbulenta manifestándose en grandes inestabilidades de los coeficientes aerodinámicos, así como también en los retrasos de la entrada en pérdida relacionados con el inicio de la formación y desprendimiento del vórtice de borde de ataque, también conocido como vórtice de pérdida dinámica. Por otro lado, Kim 2016 al analizar el efecto de la turbulencia incidente, indica que la turbulencia incidente suprime la burbuja de separación que aparece en un perfil en condiciones estáticas y flujo laminar, lo cual está producido por la gran cantidad de momento que posee el flujo turbulento, retrasando la ocurrencia del gradiente inverso de presiones en la cara de 
succión del perfil (extradós). Sus resultados muestran que no hay diferencias en las fuerzas y momentos producto de la turbulencia, ni en los ángulos de máxima sustentación, resistencia o mínimo momento. Las magnitudes de máxima resistencia y mínimo momento de cabeceo se disminuyen levemente con el incremento de la turbulencia. El punto más importante de diferencia en la influencia de la turbulencia en la dinámica ocurre durante el decrecimiento del ángulo de ataque, donde la sustentación se incrementa evidentemente cuando se incrementa la intensidad de turbulencia. El incremento promedio respecto al caso laminar es de 0,2 en el $\mathrm{Cl}$. Otro efecto evidente es que el re-pegado del flujo ocurre mucho antes que en el caso laminar. Al observar las visualizaciones, la turbulencia rompe la burbuja de separación, por lo que la región de flujo separado tiende a decrecer y el re-pegado ocurre antes. Esto lleva a un incremento de la sustentación durante el descenso del ángulo de ataque. Amendolese, en 2004, ensayó en túnel de agua un perfil NACA 634412 en niveles de turbulencia de $1 \%, 4,5 \%$ y 7,5\% midiendo presiones en la superficie del modelo. Encontró que al incrementar la turbulencia, tanto el sobre pico porcentual (respecto al valor de $\mathrm{Cl}$ en estático) del ensayo dinámico como el retraso temporal de la entrada en pérdida disminuyen al aumentar la intensidad de turbulencia. Es importante denotar que al normalizar el pico de $\mathrm{Cl}$, relativo a la amplitud de oscilación, esto da un valor constante, con lo cual indica que el sobre pico es proporcional a la amplitud de oscilación. Además, Chen en 1999 generó un entorno perturbado para generar una turbulencia de intensidad del 6\%. Con dichos ensayos, encontró que, para todas las frecuencias reducidas, se incrementó en magnitud el pico de succión en la presión cercana al borde de ataque por más de un 50\%, comparando entre el caso sin perturbación con los casos perturbados. Además, el pico se encuentra para valores de ángulo de ataque mayores. Para valores mayores de frecuencia reducida, la influencia impuesta por las perturbaciones en la corriente libre en la sustentación y en el momento, fueron menos significantes que para valores menores de frecuencia reducida. A su vez, los resultados en las mediciones de presión indican que el comportamiento del flujo sobre el perfil cuando hay una perturbación aguas arriba es como la del mismo perfil a mayores valores de número de Reynolds, tendiendo a promover la transición temprana de las capas de corte no estacionarias separándose del borde de ataque, por lo tanto, reduciendo la zona de separación.

Previamente, se realizaron estudios en el UIDET-LaCLyFA por Aramburu Orihuela et al.2016 sobre el efecto de la escala integral de turbulencia, con intensidad del $2 \%$, sobre un perfil aerodinámico Wortmann FX 63-137. Las escalas integrales utilizadas en dicho trabajo fueron del 12 y $22 \%$ de la cuerda del perfil, encontrando efectos de la misma en el coeficiente de sustentación para Reynolds entre 100.000 y 200.000. Los resultados muestran un incremento entre el 8 y $17 \%$ en el coeficiente de máxima sustentación, dependiendo del número de Reynolds, sin ser lineal la relación entre el aumento del coeficiente con el aumento del número de Reynolds, producto de la influencia de la escala turbulenta. A su vez, se identificó un retraso en la entrada en pérdida del perfil, al incrementar la escala turbulenta para los números de Reynolds de 150.000 y 200.000, pero no así en el caso de Reynolds 100.000. Además, incrementos en la pendiente de sustentación han sido hallados, especialmente en los Reynolds de 100.000 y 200.000.

\subsection{Motivación}

Un gran problema de la información que se puede encontrar en la bibliografía es que los micro-vehículos aéreos, por su tamaño, están pensados para volar en entornos urbanos, o a bajas alturas, es decir, dentro de la capa límite atmosférica. Es conocido, que dentro de dicha capa, además de los cambios de temperatura, los vientos poseen una alta componente turbulenta, lo cual modifica las condiciones de vuelo de estos vehículos. Por estudios anteriores, se conoce que la turbulencia incidente modifica las características aerodinámicas de los perfiles, en condiciones estacionarias, pero es muy escasa la literatura respecto a la influencia ante condiciones no estacionarias. Es allí donde este trabajo realiza un aporte a la comunidad científica e intenta ampliar el conocimiento en el área, pensando en la determinación de esfuerzos de sustentación. 


\section{“Estudio aerodinámico experimental en flujo turbulento de bajo Reynolds sobre alas con movimiento de cabeceo"}

El objetivo principal del estudio de esta tesis, es el análisis de la influencia de la turbulencia incidente sobre un ala con movimiento de cabeceo puro. La necesidad de conocer dicho efecto radica en la falta de información en la bibliografía sobre el efecto de esta ante condiciones no estacionarias. Es por esto que, tras caracterizar dos flujos turbulentos, logrado mediante grillas pasivas, se realizarán comparaciones de las cargas y visualizaciones del campo de flujo. Los estudios se realizarán sobre dos alas de alargamiento 4, que se extienden de pared a pared del túnel de viento para obtener una condición bidimensional (perfil aerodinámico). Se utilizaron dos perfiles: placa plana de 3\% de espesor con bordes agudos y un perfil simétrico de bajo número de Reynolds SD8020, construida en fibra de vidrio. Se estudiarán frecuencias reducidas entre 0,015 y 0,06 , para una oscilación entre $0^{\circ}$ y $15^{\circ}$. Este ángulo es limitado para poder tener una relación de bloqueo en el túnel de viento (TV4 del UIDET-LaCLyFA) que no sea mayor al 10\%. A su vez, este ángulo máximo es un ángulo mayor al de pérdida estática, tanto para una placa plana como el perfil elegido, con lo cual se busca maximizar los efectos no estacionarios.

\subsection{Esquema de la Tesis}

Las secciones de este trabajo de investigación se detallan a continuación:

Capítulo 1: En el primer capítulo fueron mencionados los antecedentes generales y específicos del tema, así como los objetivos propuestos en este trabajo.

Capítulo 2: Metodología de Ensayo: El capítulo se divide en 5 subsecciones, comenzando por una descripción general de las instalaciones utilizadas, así como la metodología empleada en los ensayos. A continuación, se define el procedimiento utilizado para la caracterización del flujo libre y las condiciones de turbulencias a utilizar. En la sección 2.3 se detalla la configuración e instrumental utilizado para la medición de la fuerza aerodinámica, así como un detalle del método de tarado dinámico empleado. A continuación, se muestra el instrumental y procedimiento utilizado en el análisis de la distribución de presiones en la estela, así como un detalle de los métodos estadísticos empleados y para finalizar, se detalla el esquema del arreglo utilizado para las visualizaciones de flujo.

Capítulo 3: El tercer capítulo de este trabajo está concentrado en los resultados obtenidos para los diferentes ensayos. En primera instancia se muestran y analizan los resultados obtenidos en la caracterización del flujo, utilizado posteriormente en los ensayos. En sección 3.2, se determinan la fuerza de sustentación para los dos modelos ensayados. Dentro de esta sección, encontraremos tres subsecciones: la primera, un caso de validación, utilizando el modelo de placa plana y un movimiento de cabeceo tipo rampa ascendente desde el ángulo de sustentación nula hasta un ángulo máximo de $45^{\circ}$. Se utilizó este movimiento para poder validar la metodología de tarado dinámico dada la cantidad de resultados disponibles con este movimiento. Luego se realizó una comparación entre las dos configuraciones de flujo turbulento para analizar la influencia de esta bajo estas condiciones. A continuación, en la subsección 3.2.2 se muestran y analizan los resultados para la placa plana en condiciones estáticas y dinámica oscilatoria continua. A continuación, se muestran y analizan los resultados obtenidos bajo las mismas condiciones (estática y dinámica oscilatoria continua) para un modelo alar con un perfil Selig Donovan 8020, completando así todos los resultados de medición de fuerza. En la sección 3.3 se analizan los resultados obtenidos con la medición de la estela para ambos modelos ensayados, utilizando distintos procesamientos estadísticos. Finalmente, el capítulo finaliza mostrando y analizando los resultados obtenidos mediante visualización de flujo para el modelo con el perfil aerodinámico SD8020.

Para finalizar, en el Capítulo 4 se detallan las conclusiones y discusiones sobre los análisis realizados y la línea de investigación en micro/mini vehículos aéreos. 
Luego de las conclusiones se encuentran cinco anexos, para facilitar la lectura de este trabajo, y dar soporte a los resultados expuestos. Los mismos comienzan con definiciones de conceptos generales de aerodinámica utilizados a lo largo del desarrollo de la tesis, para consulta del lector en caso de que fuera necesario, para continuar con la metodología de corrección de cargas por ensayo en túnel de viento utilizada. Después de estos dos anexos, se detalla la validación del método de tarado dinámico utilizado seguido de un análisis de repetitividad de los ensayos realizados. Para finalizar, se presenta la validación del sistema de medición de presiones en la estela diseñado. 


\section{"Estudio aerodinámico experimental en flujo turbulento de bajo Reynolds sobre alas con movimiento de cabeceo"}

\section{CAPÍTULO 2: Metodología de ensayo}

En este capítulo se detallará la metodología planteada para realizar los ensayos, así como también el detalle de los procedimientos y validaciones realizadas durante los mismos.

\subsection{Diseño experimental}

Todos los ensayos fueron realizados en el Túnel de Viento número 4 de la UIDET-LaCLyFA, perteneciente a la Universidad Nacional de La Plata. Dicho túnel de viento es de circuito abierto con una contracción en la entrada de 9:1. La sección de prueba es cuadrada, con 0,4 m de lado y su velocidad máxima de operación es de $30 \mathrm{~m} / \mathrm{s}$. Para poder realizar los montajes evitando las vibraciones de las paredes del túnel, se diseñó un pórtico exterior, que permitió alojar el modelo alar de forma horizontal a la mitad de la altura de la sección de prueba, donde a priori se conocía que el perfil de velocidades de la corriente libre tiene una distribución uniforme. Dicho pórtico fue empotrado al piso en uno de sus extremos, y vinculado mediante bulones en su otro lateral, para poder permitir el ajuste lateral del modelo dentro del túnel. A su vez, en la altura coincidente con la mitad de la altura del túnel de viento, se colocó un alojamiento de rodamiento, que permitiese, mediante unos acoples de aluminio, rotar libremente en su eje longitudinal el modelo alar, ver Figura 2.1. Sobre el alojamiento mencionado, ingresa el acople de aluminio diseñado para poder realizar la rotación de la balanza aerodinámica, solidariamente vinculada con el modelo alar. El eje de rotación del acople se puede ver sobre la parte izquierda de la Figura 2.2, mientras que en el lado derecho se puede realizar la vinculación con el modelo alar. De esta forma, el sistema de acoples con balanza, quedan fuera del túnel de viento, sin generar interferencia aerodinámica, y el modelo alar queda emplazado de pared a pared del túnel de viento para obtener una configuración bidimensional del flujo.

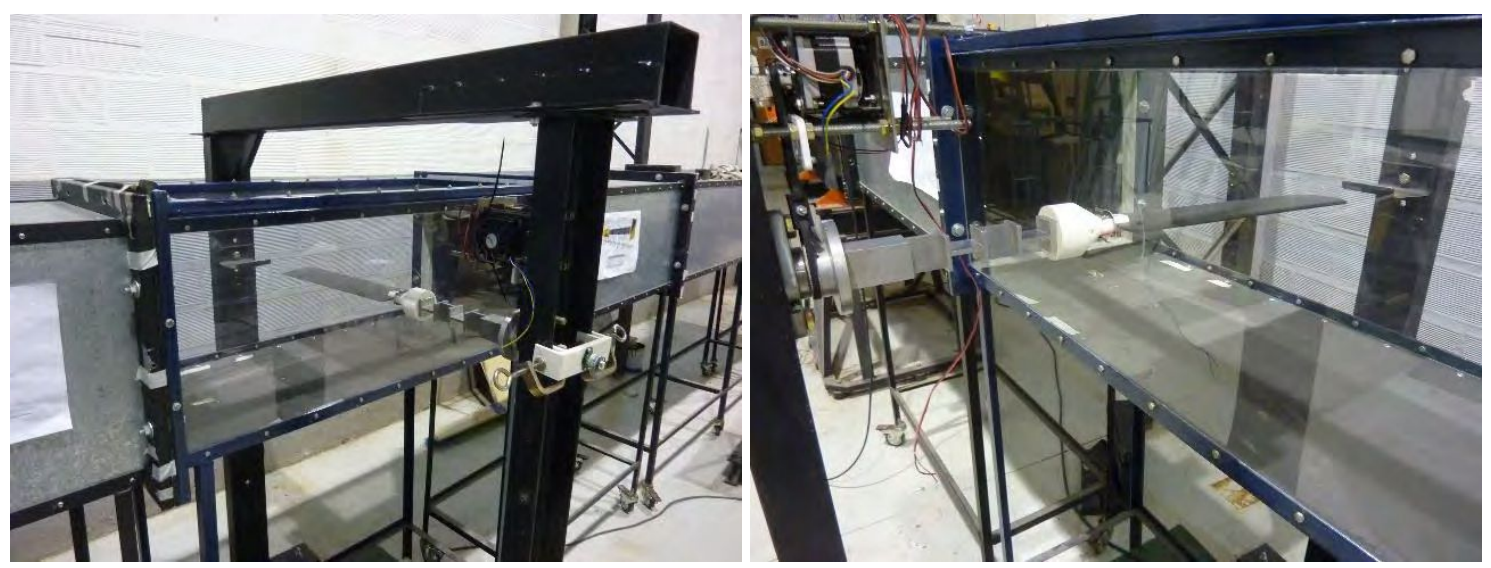

Figura 2.1. Pórtico y túnel de viento en la izquierda. Detalle del mecanismo de movimiento en la derecha.

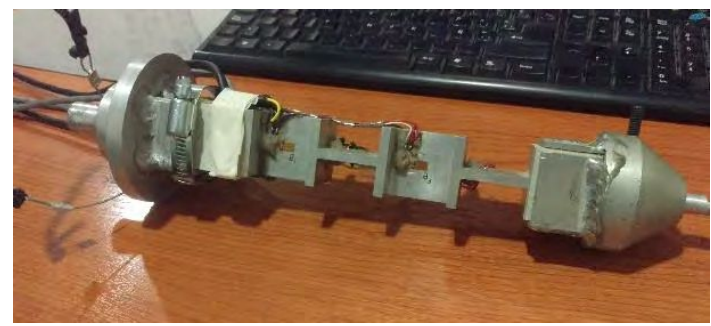

Figura 2.2. Acoples con balanza aerodinámica. 
Por otro lado, en el mismo lateral del pórtico donde se encuentra el alojamiento, se colocó un motor paso a paso NEMA 23, que mediante un sistema de poleas y correas sincrónicas (GT2), permiten realizar la rotación del conjunto Ala-Balanza (Ver Figura 2.1). Este sistema de rotación es accionado mediante un driver específico, el cual, mediante un código desarrollado en Labview, se puede accionar el sistema realizando los movimientos deseados. Dicho movimiento, es relevado mediante un sensor angular tipo Hall (RB-Dfr-726) y el software, mediante un control a lazo cerrado, realiza el movimiento del motor, si es que es necesario. Para la adquisición de dicho sensor angular se utiliza un dispositivo National Instruments NI-USB6009. Un punto importante por verificar del mecanismo es la respuesta en velocidad máxima que es capaz, limitado por el torque y velocidad de respuesta del motor, así como también con la velocidad de cálculo de la computadora que acciona el código de control. Para ello se realizaron distintos ensayos aumentando la velocidad de rotación obteniéndose que la máxima frecuencia realizable es de $0,8 \mathrm{~Hz}$, producto que si se utiliza una frecuencia de movimiento mayor el error entre el ángulo comandado y el ángulo real del ala es mayor a $1^{\circ}$, así como también desfasajes temporales considerables, lo cual generaran distorsiones en el movimiento.

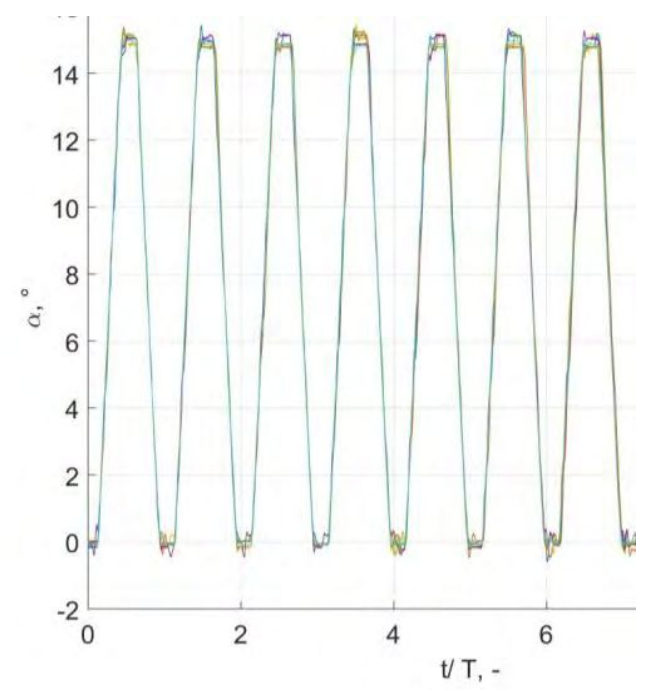

Figura 2.3. Evolución del ángulo de ataque para distintas iteraciones, en función del tiempo adimensionalizado con el periodo del movimiento, verificando la repetitividad del movimiento

Se realizaron ensayos en túnel de viento de una placa plana con borde romo que cuenta con una cuerda de $10 \mathrm{~cm}$ y una envergadura de $40 \mathrm{~cm}$ para que la misma se encuentre desarrollada de pared a pared del túnel y tener una configuración bidimensional en el flujo. El montaje comprende al agarre del ala en uno de sus extremos, siendo una configuración empotrada libre el sistema "ala-balanza", con la posibilidad de rotar por su eje central, el cual corresponde con la mitad de la cuerda del ala. El ala fue construida con una placa de aluminio de $3 \mathrm{~mm}$ de espesor, lo que corresponde con una relación espesor/cuerda del 3\%. Tanto al borde de ataque como el borde de fuga, no se le realizó ningún tratamiento para redondear sus bordes, por lo que la geometría es la de un borde recto. La balanza aerodinámica de dos componentes utilizada fue construida en el laboratorio en acero inoxidable y permite realizar las mediciones de la fuerza normal y la fuerza axial a la cuerda, según el montaje implementado en el ensayo. A su vez, se colocó un acelerómetro tri-axial (ADXL-335) y un giróscopo (SNENCOR) en el extremo del ala, sobre el eje de rotación, para poder medir el ángulo de ataque y la velocidad de giro. También, se acopló un sensor hall para poder medir el ángulo de rotación en los casos de la presencia de aceleraciones angulares (ensayos dinámicos), donde el acelerómetro no es capaz de realizar una medición precisa.

Todos los ensayos fueron realizados para dos modelos alares, que poseen la misma cuerda $(10 \mathrm{~cm})$ y la misma envergadura $(40 \mathrm{~cm})$. La envergadura fue elegida de forma tal que ocupase el ancho completo de la sección del túnel de viento, para evitar los efectos de punta de ala y tener así una condición bidimensional del 


\section{"Estudio aerodinámico experimental en flujo turbulento de bajo Reynolds sobre alas con movimiento de cabeceo"}

flujo incidente. Se dejó un huelgo de $1 \mathrm{~mm}$ entre los extremos del ala y las paredes del túnel para asegurar que no se produzcan roces entre estos lo cual generarían errores en las mediciones de fuerza. El primer modelo ensayado es una placa plana de $3 \mathrm{~mm}$ de espesor $(3 \% \mathrm{t} / \mathrm{c})$, construida en aluminio, con bordes romos. El segundo modelo, fue construido en fibra de vidrio, utilizando un perfil simétrico SD8020, el cual tiene un espesor máximo del $10 \%$ de la cuerda, y su distribución de espesores está optimizada para bajos números de Reynolds. El principal objetivo del análisis de estos dos modelos alares es estudiar el efecto del borde de ataque y de la distribución de espesores. En la figura siguiente se muestran dos fotografias de los modelos alares donde se visualizan las diferencias en la geometría. Si bien todos los modelos construidos tienen la posibilidad de modificar su punto de rotación en la dirección de la cuerda, para este trabajo el punto de rotación ha sido fijado en la mitad de la cuerda, dado que esta ubicación minimiza los efectos de masa aparente, y ya es conocido que el cambio en el punto de rotación solo genera un efecto de retraso en la formación del LEV, pero no así en las cargas máximas.
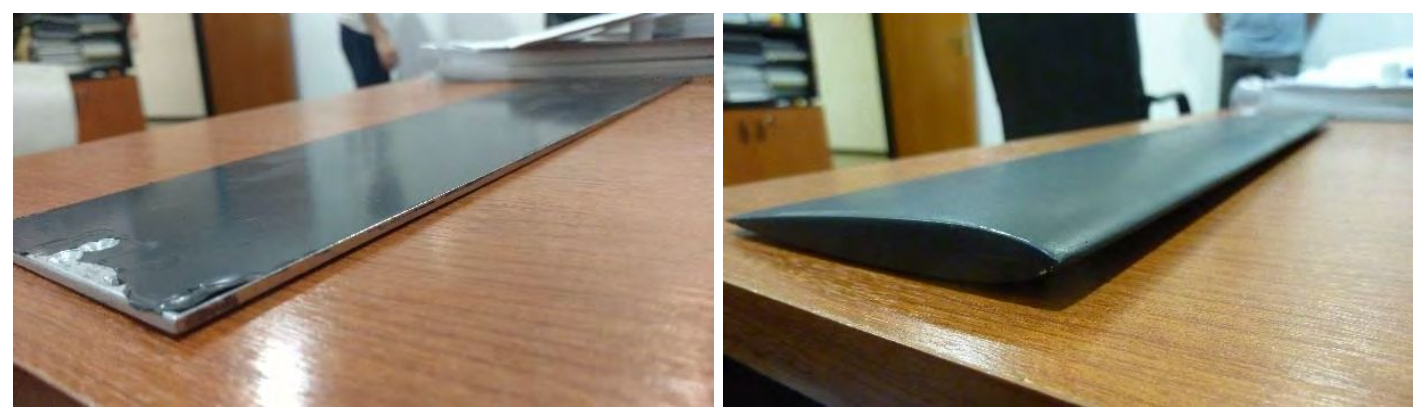

Figura 2.4. Modelos Alares utilizados. En la izquierda placa plana, en la derecha perfil SD8020

\subsection{Caracterización de la corriente libre}

Mediante el uso de anemometría de hilo caliente, se realizaron mediciones del perfil de velocidades en la sección de prueba del túnel de viento utilizado. Dichas mediciones se realizaron en dos condiciones: con flujo "limpio" y con una grilla que genera turbulencia adicional. La condición de flujo limpio se considera a la del túnel de viento sin ningún elemento extra, es decir, la condición del flujo dentro del túnel sin ninguna perturbación. Para aumentar la intensidad de turbulencia, y modificar las escalas, se agregó justo después de la contracción de entrada una grilla plástica que posee cuadrados de $15 \mathrm{~mm}$ de lado aproximadamente, y un espesor de 3 a 4 mm. Ver Figura 2.6

Para las mediciones de velocidad se utilizó la técnica de anemometría de hilo caliente, con el sistema CTA Streamline de Dantec Dynamics, utilizando un sensor doble "fiber film" 55R51. La adquisición se realizó a 600 $\mathrm{Hz}$, utilizando un filtro pasa-bajo a $300 \mathrm{~Hz}$, obteniendo 16 muestras por cada velocidad y punto ensayado. Se realizó un barrido en altura comenzando en $50 \mathrm{~mm}$ respecto a la pared inferior del túnel hasta una altura de $350 \mathrm{~mm}$, todas las mediciones en la posición central respecto al plano transversal (se recuerda que la altura máxima de la sección de prueba es de $400 \mathrm{~mm}$ ). Estas distancias representan, medidos desde la altura donde se emplazó el modelo alar, una distancia de una cuerda y media hacia arriba y abajo. 

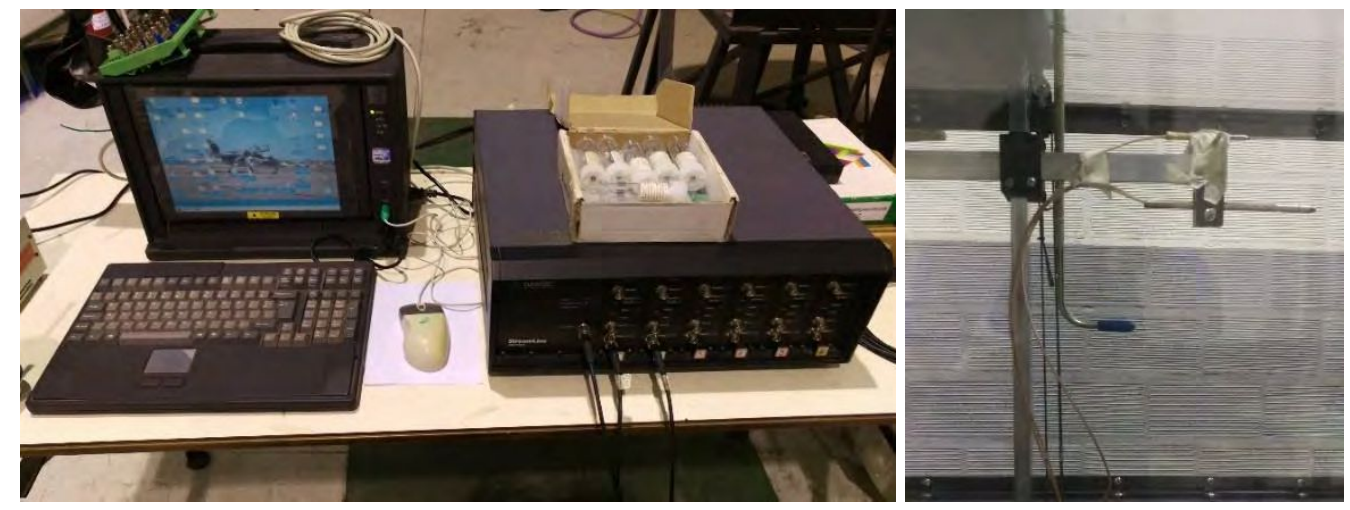

Figura 2.5. Instrumental utilizado en la izquierda. Montaje en la sección de prueba del sensor anemométrico.

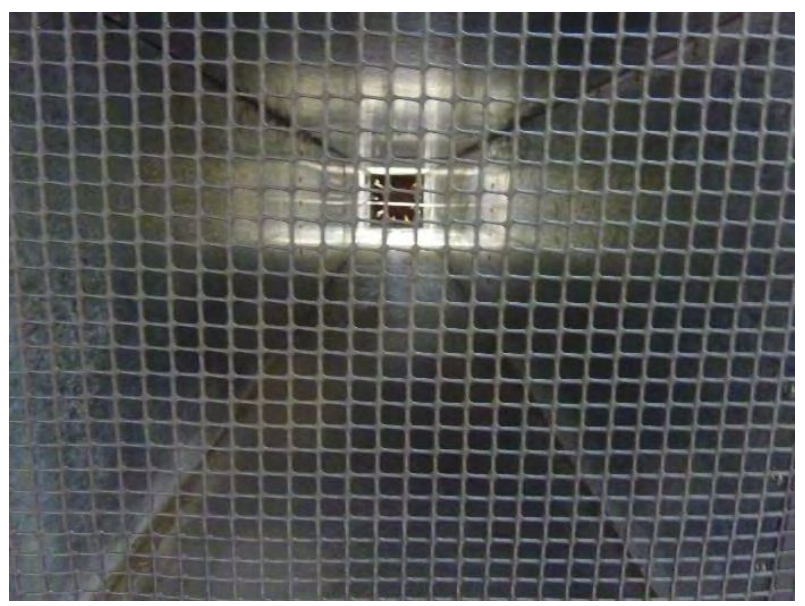

Figura 2.6. Grilla generadora de turbulencia localizada luego de la contracción del túnel de viento.

Con las mediciones realizadas, se obtuvieron los perfiles de velocidad media, en su componente longitudinal "u", y en su componente vertical "v". A su vez, se calculó la intensidad de turbulencia en cada componente y las escalas integrales espaciales y temporales de la turbulencia para cada componente. Es de especial interés que el perfil de velocidades tenga un frente plano en la ubicación donde se emplazará el modelo. La intensidad de turbulencia fue calculada con su expresión clásica, en función de la media cuadrática de la fluctuación, aunque considerando tanto para la componente " $\mathrm{u}$ " y " $\mathrm{v}$ " el valor medio del vector velocidad como valor de referencia. Ver fórmulas a continuación para el detalle.

$$
\begin{aligned}
& I_{u}=\frac{u_{r m s}^{\prime}}{\sqrt{\bar{u}^{2}+\bar{v}^{2}}} \\
& I_{v}=\frac{v_{r m s}^{\prime}}{\sqrt{\bar{u}^{2}+\bar{v}^{2}}}
\end{aligned}
$$

Para la definición de las escalas integrales de la turbulencia, se utilizó el valor temporal para el cual el coeficiente de auto correlación toma el valor de 1/e, el cuál refiere a un tamaño promedio de los vórtices que se encuentran en el flujo (Handbook of Experimental Fluid Mechanics, Springer). Mediante la teoría de flujo congelado (Taylor, 1938), dicho valor temporal de la auto correlación, junto con la velocidad media del flujo en ese punto, se puede estimar el tamaño medio de los vórtices. Por último, pero por ello no menos importante, se calcularon los espectros de potencia utilizando la transformada de Fourier, para poder comparar las energías de la velocidad en frecuencias. 


\section{"Estudio aerodinámico experimental en flujo turbulento de bajo Reynolds sobre alas con movimiento de cabeceo"}

\subsection{Mediciones de fuerza}

Primeramente, se realizaron ensayos estáticos realizando un barrido de ángulo de ataque iniciando desde - $2^{\circ}$ hasta $45^{\circ}$ para el modelo de placa plana, y para el modelo con perfil aerodinámico se realizó hasta $21^{\circ}$ de ángulo máximo. Estos ensayos se realizaron para distintas velocidades de corriente libre, siendo estas 4, 8 y $10 \mathrm{~m} / \mathrm{s}$ correspondiendo respectivamente con un número de Reynolds basado en la cuerda de 23.000, $46.000 \mathrm{y}$ 57.000 aproximadamente.

Luego, se realizaron ensayos de la placa plana con movimiento de cabeceo puro (rotación respecto a un eje paralelo al borde de ataque). Para ello, se realizaron ensayos con un movimiento del tipo rampa, de $0{ }^{\circ}$ a $45^{\circ}$ de ángulo de ataque, con una función suavizante para minimizar la aceleración de rotación, la cual involucra cargas inerciales que se intentan minimizar para facilitar las mediciones. Para ello se utilizó la función desarrollada por Eldredge, 2016 y ensayada por distintos laboratorios, lo cual sirve de comparación y validación de los ensayos obtenidos. Este tipo de movimiento se puede considerar un movimiento canónico en función de la cantidad de laboratorios que lo han ensayado, generando así una gran base de datos para poder comparar resultados.

Para finalizar, se repitieron los mismos ensayos con un nivel de turbulencia distinto y mayor al realizado inicialmente para poder comparar y analizar la influencia de esta en las cargas aerodinámicas estáticas y dinámicas.

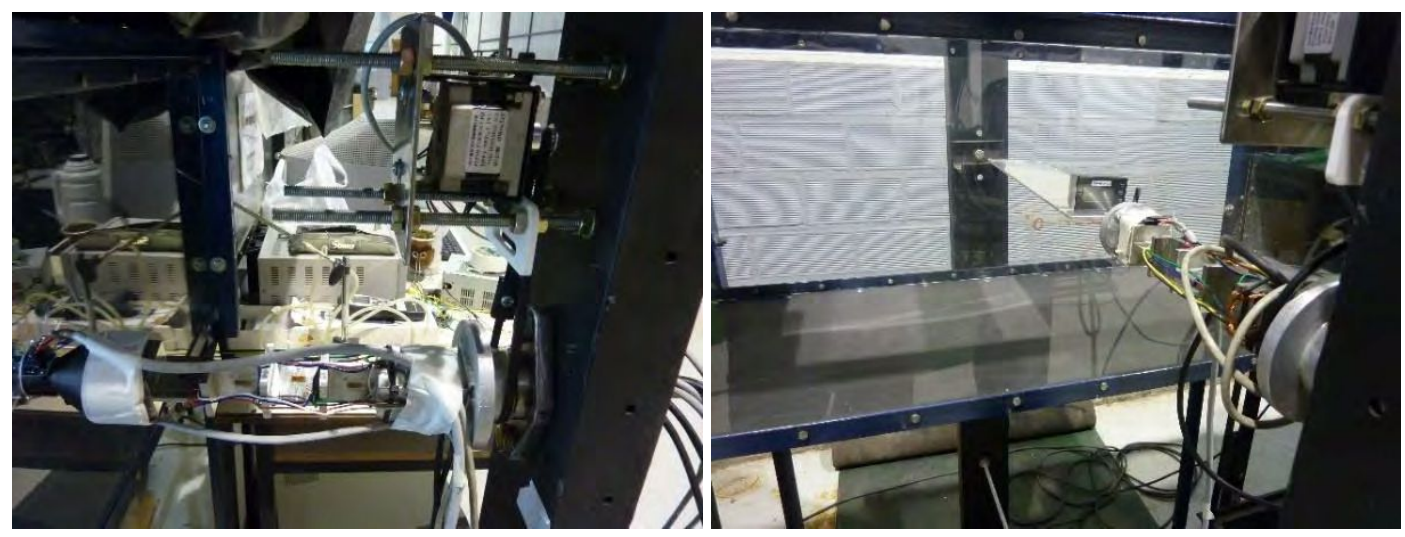

Figura 2.7. Montaje en túnel de viento del ala con su sistema de rotación.

A su vez, para poder analizar un modo más cercano al desarrollado por las aves en sus vuelos, se analizó un movimiento continuo de tipo rampa ida y vuelta. Utilizando la misma metodología empleada para el movimiento del tipo rampa, se completó el ciclo con una rampa descendente y se realizó el ensayo para un movimiento continuo a distintas frecuencias reducidas, limitando el ángulo máximo alcanzado a un valor de $15^{\circ}$ para este movimiento. Este ángulo final fue elegido en función de dos parámetros: que el ángulo final sea mayor al ángulo de pérdida estática para maximizar los efectos no estacionarios, y para tener un bloqueo menor al $10 \%$. Mayor detalle sobre la determinación del bloqueo y las correcciones implementadas se pueden encontrar en el Corrección por mediciones en túnel de viento. La misma función desarrollada por Eldredge, 2016 fue empleada para suavizar la aceleración desarrollada por el ala al realizar su movimiento de rotación. Continuando con una placa plana, se realizaron mediciones de las cargas aerodinámicas, empleando el sistema de tarado dinámico mediante elemento equivalente y se compararon los resultados obtenidos ante las mismas configuraciones de turbulencia desarrolladas en el ensayo de caracterización de la corriente libre.

En este caso, la frecuencia reducida fue calculada mediante la expresión ( 1.3 ), lo cual está relacionado con el número de Strouhal calculado mediante la expresión ( 1.2 ). Con las capacidades del mecanismo 
generador del movimiento, se analizaron tres frecuencias reducidas: 0,015, 0,03 y 0,06 las cuales representan número de Strouhal de 0,0047, 0,009 y 0,019, respectivamente. Para las velocidades de corriente libre de $8 \mathrm{~m} / \mathrm{s}$ solo se analizan las dos frecuencias reducidas menores, mientras que para la máxima velocidad se estudió con la mínima frecuencia reducida.

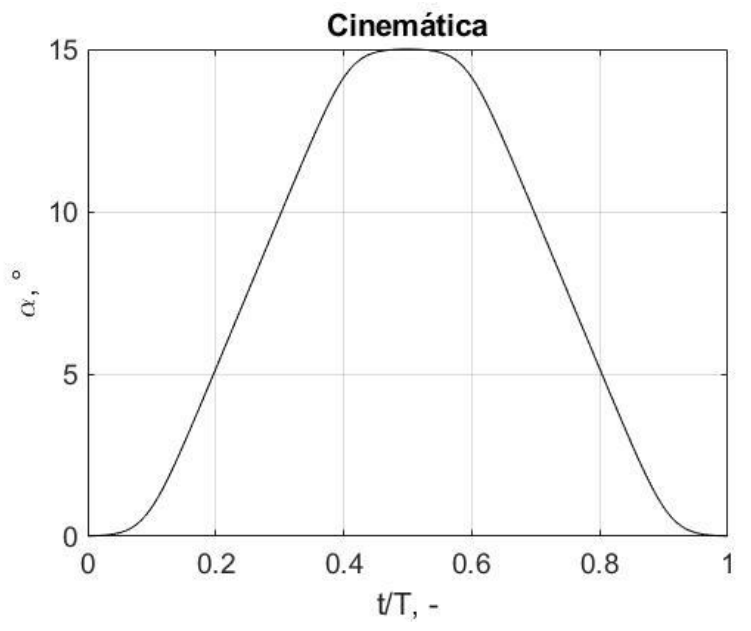

Figura 2.8.Evolución del ángulo de ataque en el tiempo adimensionalizado con el periodo, en el modo continuo.

Se realizaron mediciones con balanza aerodinámica del ala cambiando su ángulo de ataque siguiendo el ángulo como se muestra en la Figura 2.8 para los tres números de Reynolds especificados con anterioridad, y las frecuencias reducidas seleccionadas. Al igual que para el ensayo con movimiento tipo rampa, se empleó un filtro digital Chebyshev del tipo II para eliminar las vibraciones mecánicas de las mediciones de cargas. En la Figura 2.9 se muestra un ejemplo de cómo es la señal adquirida, y el resultado tras aplicarle el filtro digital. En la misma figura se puede observar la secuencia utilizada para las mediciones. En primera instancia, se deja durante un segundo un ángulo de ataque fijo igual a $0^{\circ}$, y luego se repiten 11 ciclos de rampa ida y vuelta, para luego promediar el valor de los ciclos, eliminando el último ciclo. A su vez, este procedimiento se repitió 5 veces para cada condición, con lo cual se obtienen las mediciones de 50 ciclos, los cuales fueron promediados para obtener los valores medios correspondientes.

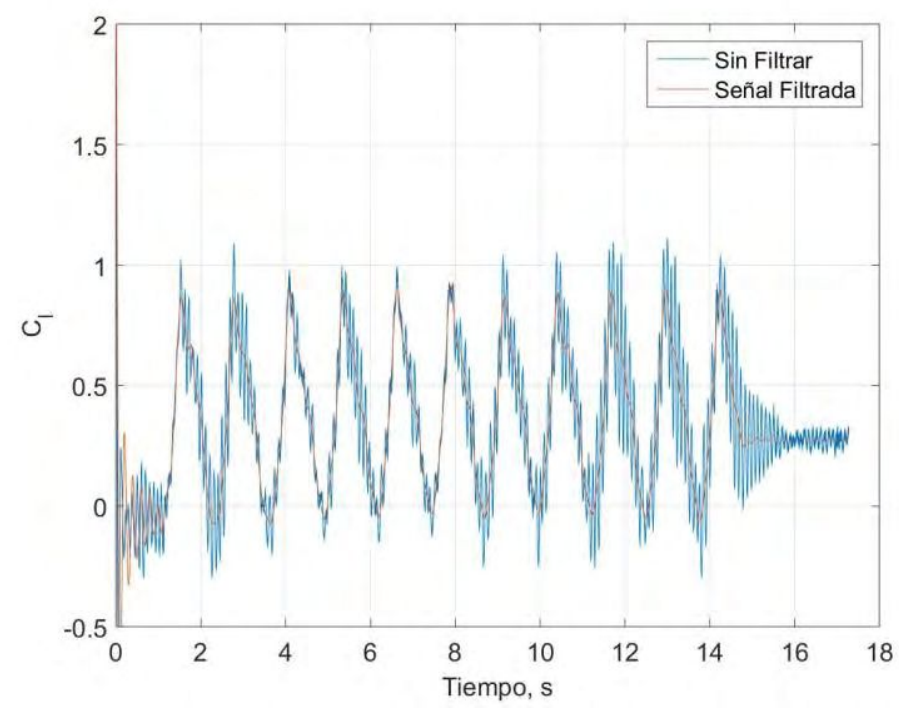

Figura 2.9. Señal sin procesamiento de filtrado, y filtrada para eliminar vibraciones mecánicas. 


\section{"Estudio aerodinámico experimental en flujo turbulento de bajo Reynolds sobre alas con movimiento de cabeceo"}

En las mediciones experimentales de la fuerza aerodinámica, es necesario primero medir las fuerzas gravitacionales e inerciales que genera el movimiento propio del ala, ya que estas pueden ser del mismo orden, incluso mayores, que las fuerzas aerodinámicas de interés. Para las mediciones en ángulo de ataque estático, se consideró el peso del modelo alar directamente como fuerza de tarado, obtenido previamente para un ensayo sin velocidad de viento, pero esta metodología no es adecuada cuando el modelo está rotando con cierta velocidad, y aceleración, con lo cual se debió generar una metodología para medir los esfuerzos inerciales en la condición dinámica. Algunos investigadores han realizado estimaciones mediante modelos del tipo CAD para estimar las inercias del ala, y luego, conociendo el movimiento que están estudiando, calculan las fuerzas inerciales producidas sobre la balanza y así restarlas a la medición. Otros, sin embargo, han determinado que las fuerzas inerciales y gravitacionales como un error sistemático en la medición, por lo tanto, determinaron estos valores de forma analítica y mediante métodos computacionales (Isaac et al. 2006). Otro método utilizado, es el de generar un elemento equivalente, de igual masa e inercia que el ala a ensayar, pero que minimice la fuerza aerodinámica generada, con lo cual, realizando mediciones con dicho elemento se pueden obtener las fuerzas inerciales durante cada momento del movimiento y luego realizar la resta respecto a las fuerzas medidas con el ala. Para este trabajo se analizaron diferentes metodologías, para poder cuantificar los efectos inerciales. Finalmente, se decidió construir un elemento equivalente de igual masa, inercia y ubicación del centro de gravedad que cada modelo alar, pero que minimice la fuerza aerodinámica generada al moverse con la misma cinemática que el ala. Mayor detalle de las mediciones y determinación del modo de tarado dinámico se puede encontrar en el Validación del método de tarado dinámico. Básicamente, este modelo es un cilindro con un brazo transversal y contrapesos, que permiten localizarse según las necesidades de cada modelo alar, dado que la placa plana y el modelo en fibra de vidrio con perfil aerodinámico no poseen exactamente la misma masa, inercia y centro de gravedad. Al ser un cilindro, la fuerza aerodinámica desarrollada es despreciable, sobre todo dado que el ensayo fue realizado sin viento incidente, para minimizar dicho efecto y solo poder medir los esfuerzos inerciales.

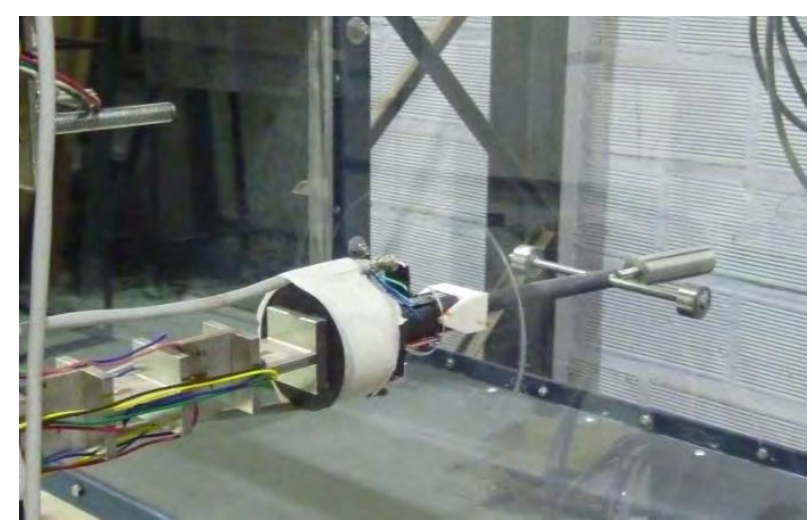

Figura 2.10. Elemento equivalente montado en el túnel de ensayo

\subsection{Mediciones de presión en estela}

Además de las mediciones de fuerza aerodinámica, se realizaron mediciones mediante un rack de presiones diseñado para tal fin, para la caracterización del campo de flujo en la estela del perfil. A continuación, se detallarán las características constructivas del rack de presiones diseñado para la medición de la estela en el Túnel de Viento TV4 de la UIDET-LaCLyFA.

Para el diseño del rack se consultó bibliografía referente a las mediciones de estela de perfiles, (Barlow et al. 1990, Handbook of Experimental Fluid Mechanics y NACA TN 2520). En ellos, se especifican las buenas prácticas a tener en cuenta a la hora de diseñar este tipo de instrumentos, así como la implementación de esta 
técnica de medición para la determinación de la resistencia aerodinámica a partir de la integración de la cantidad de movimiento, procesamiento que excede el alcance de este trabajo, aunque se empleó para la validación del instrumento, consultar el Validación de mediciones mediante Rack de Presiones.

Para el diseño del rack de presiones, se decidió implementar la construcción mediante impresión 3D (impresión mediante el modelado de deposición fundida de plástico), de una extrusión de un perfil simétrico NACA de $18 \%$ de espesor. Sin embargo, no se utilizó el perfil completo, sino que se consideró únicamente desde borde de ataque hasta el punto de máximo espesor (30\% de la cuerda aproximadamente). A dicho soporte, se le definieron los 31 puntos de medición de presión total para medir en forma simultánea. El espaciado entre tomas de presión no es uniforme, de forma tal de concentrar la mayor cantidad de sensores en la cercanía al eje de rotación del ala. De esta forma se logra un mayor detalle donde se espera tener mayor gradiente de velocidades, en función de los resultados publicados por los autores de referencia citados anteriormente. A su vez, la toma de presión, fabricada con tubo de bronce-latón de $1 / 16^{\prime \prime}$ de diámetro, se separa del soporte por $4,5 \mathrm{~cm}$, lo que equivale aproximadamente a cuatro veces el largo del soporte de las tomas de presión, para mitigar los efectos de interferencia.

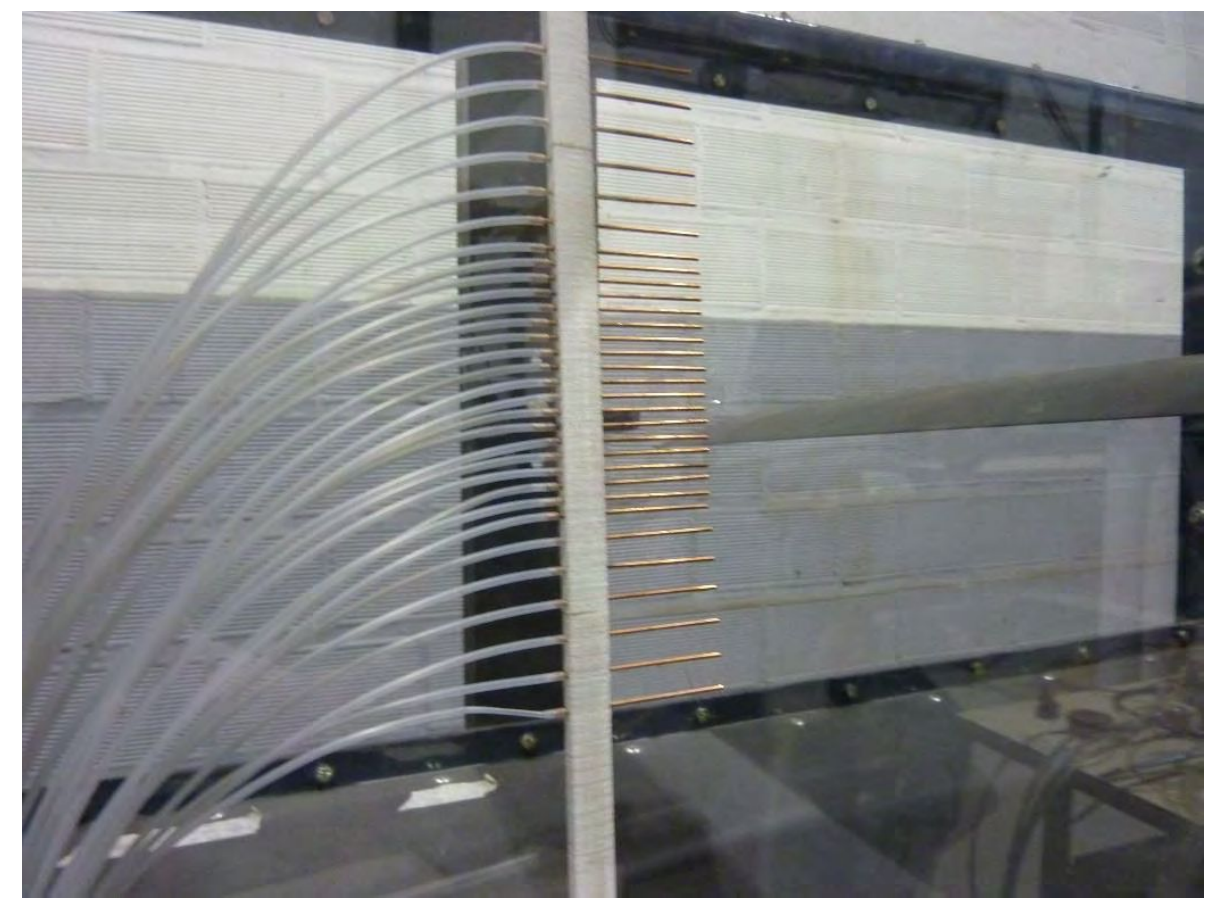

Figura 2.11. Rack de presiones ubicado en la sección del túnel de viento

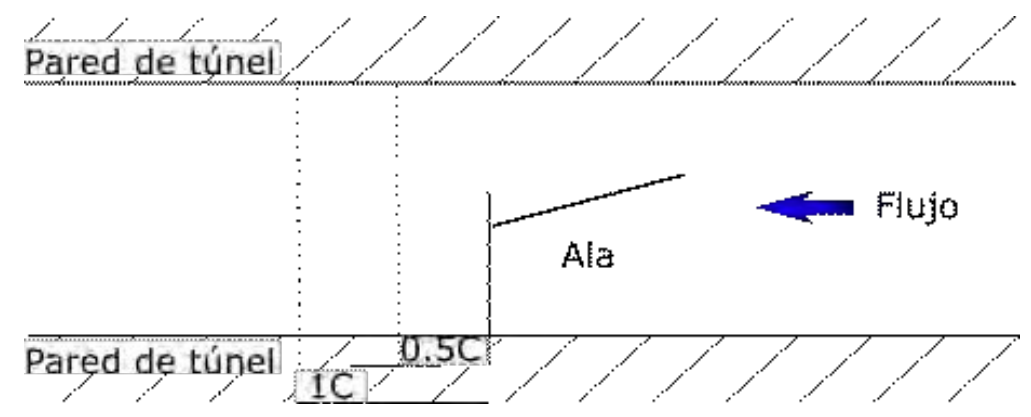

Figura 2.12. Estaciones de medición de estela. 

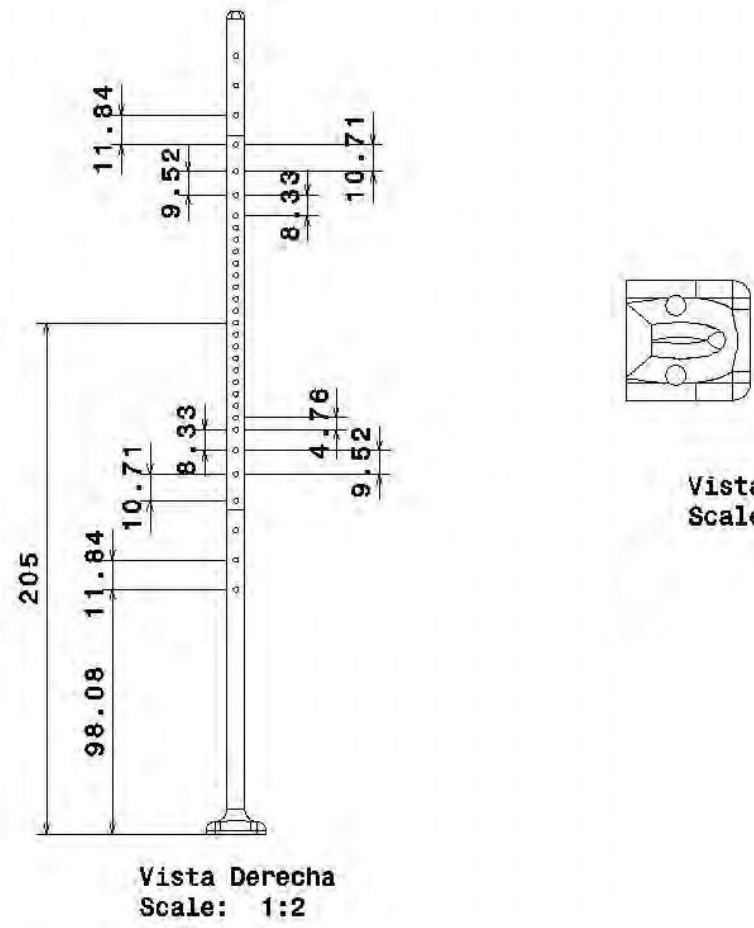

Vista Superior Scale: $1: 1$

Figura 2.13. Esquema con medidas del rack de presiones. Las medidas no mostradas corresponden a una medida idéntica a la inferior más cercana. Todas las dimensiones son en milímetros.

Dicho rack fue colocado a dos distancias medidas desde el borde de fuga del perfil con el ala en $0^{\circ}$ de incidencia, equivalentes a media y una cuerda del perfil, para realizar las mediciones correspondientes en la estela del modelo, ver esquema en la Figura 2.12. Se realizaron mediciones en dos estaciones aguas abajo para poder realizar correlaciones cruzadas entre estas y así tener un análisis temporal del transporte de vórtices en la estela del fluido. Para evitar interferencia en las mediciones de presión, se decidió no realizar las mediciones en simultáneo, sino que se realizó en mediciones diferentes, pero sincronizando la adquisición de las presiones con el movimiento del mecanismo de movimiento. De esta forma, es posible asegurar que las adquisiciones están sincronizadas temporalmente, relacionadas al movimiento del ala, y pueden ser comparadas y correlacionadas entre sí. Las adquisiciones fueron realizadas con dos escáneres de presión Scanivalve DSA 3217, a su frecuencia máxima de adquisición de $500 \mathrm{~Hz}$, mediante un software desarrollado en Labview para tal fin. El sincronismo de la señal de gatillo de los escáneres de presión fue realizado mediante una llave electrónica.

\section{Correlación cruzada}

Una forma estadística de determinar la similitud entre dos señales, para poder encontrar eventos relevantes que ocurren en dos sensores, es la utilización de la correlación cruzada, también conocida como covarianza. Esta función es dependiente del tiempo relativo de las señales, y comúnmente es expresado como un coeficiente adimensional, donde se normaliza el valor de la correlación con el valor para retardo cero de la auto correlación (correlación de la misma señal consigo misma). Un caso particular de la correlación cruzada es la correlación de la misma señal consigo misma, denominada auto correlación, muy utilizada en el campo de la fluidodinámica para poder determinar escalas turbulentas. En particular, para este trabajo, las correlaciones cruzadas y auto correlaciones serán utilizadas para poder comparar las mediciones de presión entre dos sensores, así poder determinar la similitud entre estas señales, y con el retardo, poder estimar el tiempo de pasaje de vórtices.

La expresión matemática que determina la correlación cruzada entre dos señales f y g es:

$$
(f * g)(\tau)=\int f^{*}(t) g(t+\tau) d t
$$


Siendo $\tau$ el retardo en la señal. Para el caso de la normalización, se utiliza el valor obtenido de aplicar la ecuación anterior para $\mathrm{g}=\mathrm{f}$ y retardo $(\tau)=0$. El considerar que la función $\mathrm{g}=\mathrm{f}$, nos pone en el punto de estar evaluado la auto correlación.

\subsubsection{Transformada de Fourier}

La transformada de Fourier, es un procedimiento matemático para transformar una señal del espacio tiempo al espacio de frecuencias y su operación inversa. Para el caso de señales discretas, el método comúnmente empleado es el de la Transformada Rápida de Fourier, el cuál es un algoritmo optimizado para realizar el cálculo de la transformada de Fourier en una señal discreta. Principalmente, en el procesamiento de señales en ingeniería o física, es empleada para la determinación de eventos periódicos, así como el análisis de la energía de dicha señal en una componente específica de frecuencia. La determinación de la transformada rápida de Fourier se realiza mediante el comando "FFT" del software Matlab, el cual determina la señal Y (k) en el dominio de las frecuencias de una función X mediante la siguiente ecuación:

$$
Y(k)=\sum_{j=1}^{n} X(j) W_{n}^{(j-1)(k-1)}
$$

Donde

Es una de las "n" raíces de la unidad.

$$
W_{n}=e^{-\frac{2 \pi i}{n}}
$$

\subsubsection{Transformada de Wavelet}

Otra herramienta ampliamente utilizada para el análisis de señales no estacionarias es el de la técnica de la transformada continua con Wavelet (CWT), la cual permite analizar en el espacio tiempo-frecuencia una señal, mediante la traslación y dilatación de una "onda madre". En este caso, se realizó el análisis utilizando la segunda derivada de la función de Gauss, la cual nos permite localizar vórtices de distintas escalas en las señales, dada la forma que posee la misma (Farge 1992, Farge \& Schneider 2006). Una de las ventajas de la utilización de este método es que permite localizar en frecuencias y también en el tiempo de la señal, aunque perdiendo precisión en la determinación del tiempo y frecuencia.

Específicamente en flujos turbulentos, Farge, 1992 propuso un método basado en análisis en Wavelets para extraer estructuras coherentes para flujos turbulentos, tanto en 2D como en 3D. Este método principalmente se focaliza en la vorticidad más que en la velocidad.

En líneas generales, el método consiste en utilizar una onda de forma conocida (wavelet), que se puede localizar en espacio y longitud de onda, y compararla a distintos tiempos y amplificaciones con respecto a la señal a analizar. En comparación con Fourier, el procedimiento es muy similar, salvo que Fourier utiliza funciones senos y cosenos, que están perfectamente ubicadas en el espacio de frecuencias (longitud de onda), pero no en el espacio temporal. Para más información sobre la aplicación del método en identificación de flujos turbulentos se puede consultar Farge y Schneider 2016, o Schneider y Vasilyev 2010. 


\section{"Estudio aerodinámico experimental en flujo turbulento de bajo Reynolds sobre alas con movimiento de cabeceo"}

\section{$2.5 \underline{\text { Visualizaciones }}$}

Mediante la implementación de un arreglo de lentes ópticas, se generó a partir de un haz de luz de color rojo o verde, un plano de luz con el fin de iluminar la sección central del perfil, de forma tal de observar el comportamiento bidimensional del flujo, ver Figura 2.14. Se realizaron visualizaciones generando una nube de humo que abarcaba la sección por completo, mediante el uso de una máquina de humo construida en el laboratorio para tal fin. El plano iluminado tiene la finalidad de solo visualizar la sección deseada, omitiendo las secciones no iluminadas. De esta forma, se logró capturar el comportamiento del fluido en torno al modelo alar para distintos ángulos de ataque, además del comportamiento cualitativo del flujo al realizar el movimiento de cabeceo. Se tomaron fotografías de alta calidad y videos en alta definición a una velocidad de 60 cuadros por segundo, utilizando una cámara fotográfica LUMIX - Panasonic - DMC-ZS3. A partir de los videos se extrajeron los distintos cuadros de la filmación para poder analizar el movimiento del flujo cuadro a cuadro, realizando un post-procesamiento de las imágenes mediante el uso del software Matlab, con su librería para tal fin. En la Figura 2.15 se puede observar, en una imagen sin editar, los principales componentes de la técnica de visualización a modo introductorio de la técnica utilizada.

Todas las imágenes obtenidas para la visualización mediante técnica de humo, fueron realizadas a una velocidad de corriente libre de $4 \mathrm{~m} / \mathrm{s}$ lo que corresponde con un número de Reynolds de 23.000 en función de la cuerda del ala. No se realizaron visualizaciones a mayor velocidad porque con la velocidad de filmación disponible, y la potencia del láser, no era posible realizaron visualizaciones lo suficientemente claras para un análisis correcto de las imágenes.

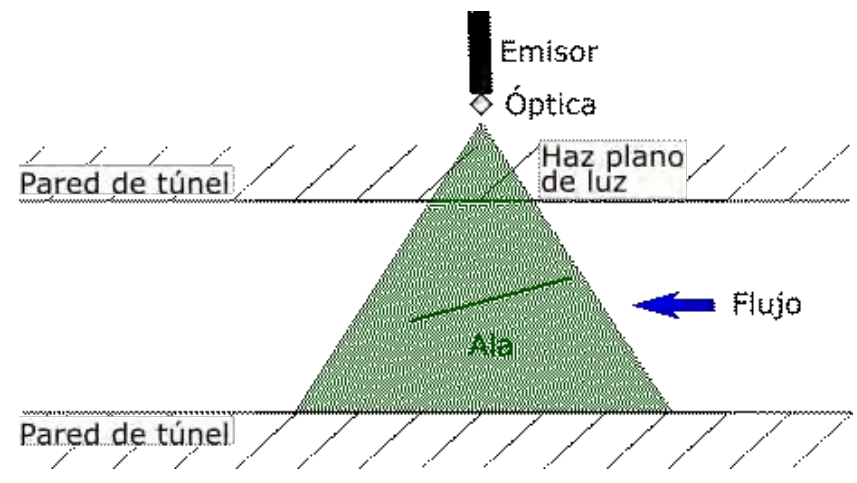

Figura 2.14. Esquema del montaje del arreglo óptico para generación de haz de luz.

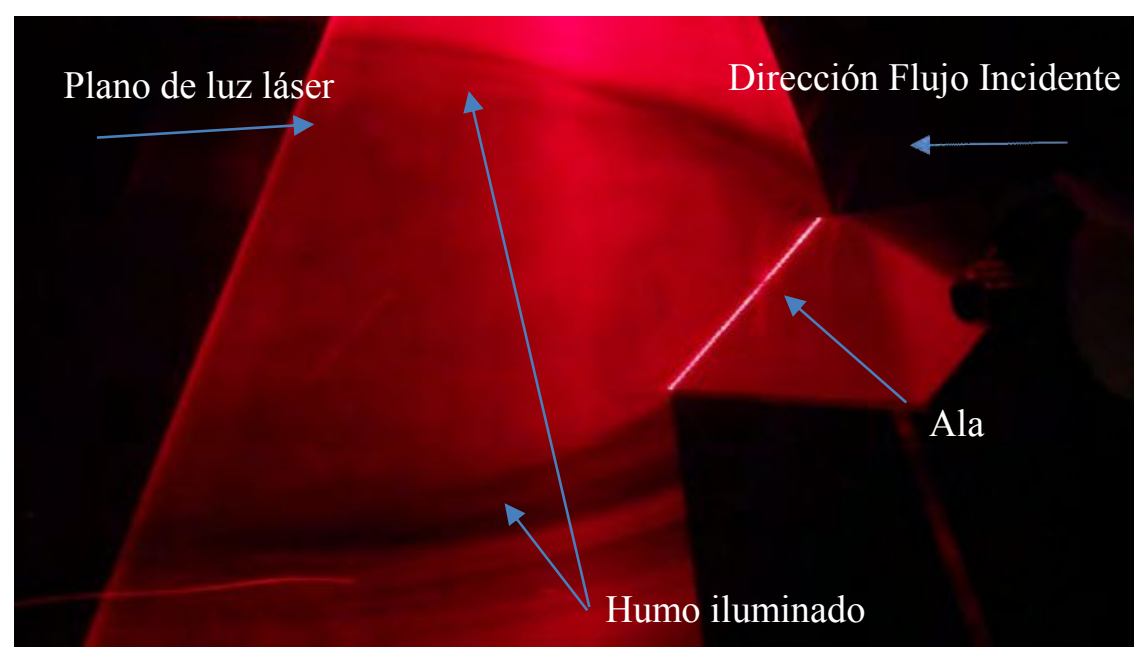

Figura 2.15. Demostración de visualización de flujo mediante humo y plano de luz láser 


\section{CAPÍTULO 3: Resultados y Análisis}

\subsection{Caracterización del Túnel de Viento}

Para comenzar con el análisis de la caracterización del flujo incidente en el túnel de viento, se procedió a graficar el perfil de velocidades medias en sus dos componentes (dado que la componente lateral no ha sido estudiada). En el perfil de velocidades longitudinal ("u") se puede observar un perfil plano de velocidades a partir de los $70 \mathrm{~mm}$ hasta los $330 \mathrm{~mm}$ de altura de la sección del túnel para ambas velocidades. Las velocidades representadas se encuentran adimensionalizadas con la velocidad de referencia, ver Figura 3.1. En ambos gráficos se visualizan los resultados hallados para dos configuraciones diferentes, una configuración libre (grilla 0 ), y la configuración con una grilla generadora de turbulencia de $15 \mathrm{~mm}$ de apertura (grilla 15). Al observar el perfil de velocidades en la componente vertical ("v"), presenta valores menores al $2 \%$ de la componente longitudinal en la zona central de la sección de prueba, lo que representa un valor en ángulo de ataque próximo a $0,5^{\circ}$, donde se calcula dicho ángulo como el atan $(\bar{v} / \bar{u})$, ver Figura 3.2. Mayor detalle sobre el análisis del ángulo de ataque se podrá encontrar a continuación de las figuras de los perfiles de velocidad.

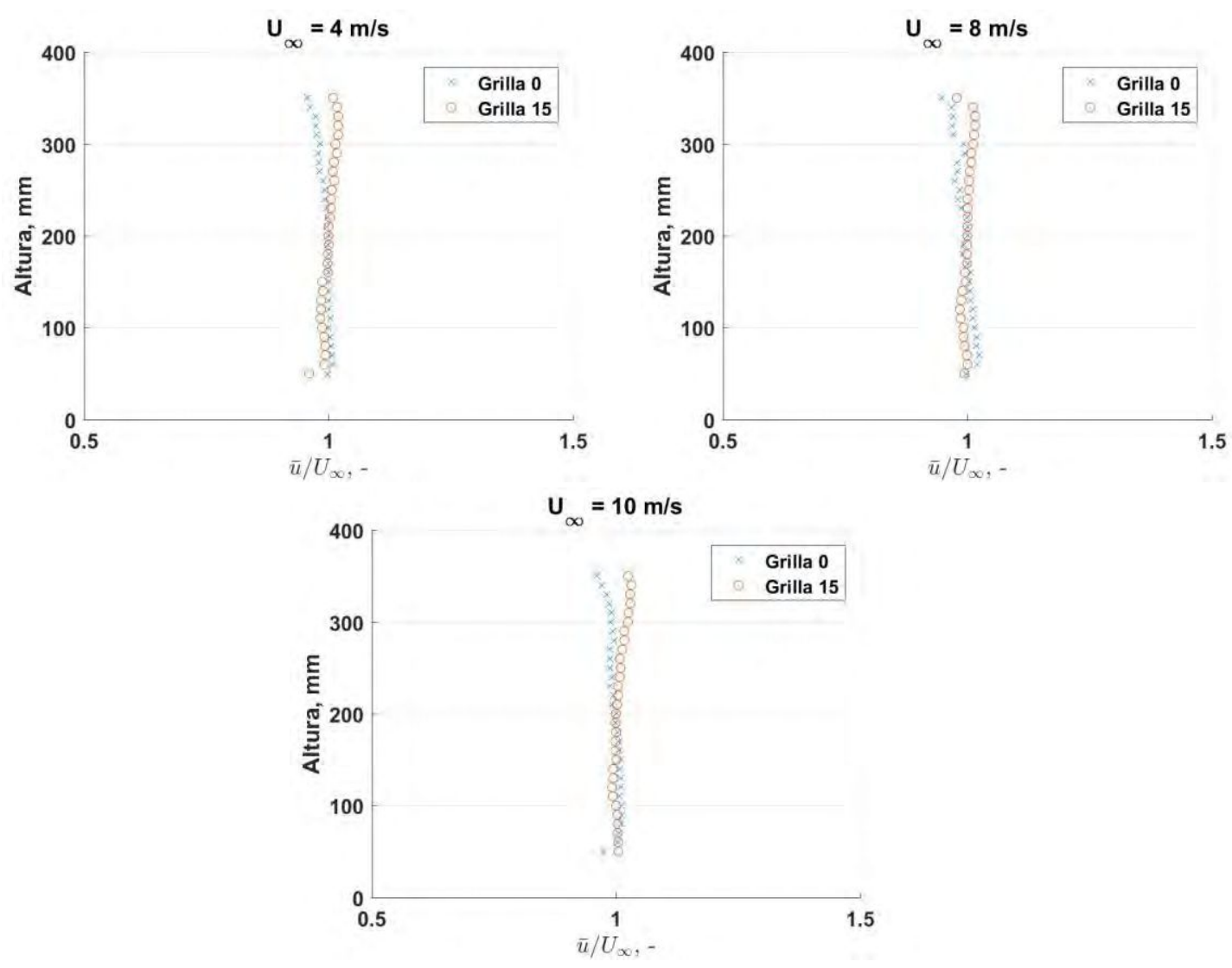

Figura 3.1. Velocidad longitudinal media para cada grilla de turbulencia adimensionalizada con la velocidad de referencia. “Grilla 0" indica sin grilla, “Grilla 15” indica grilla con apertura de 15 mm. 

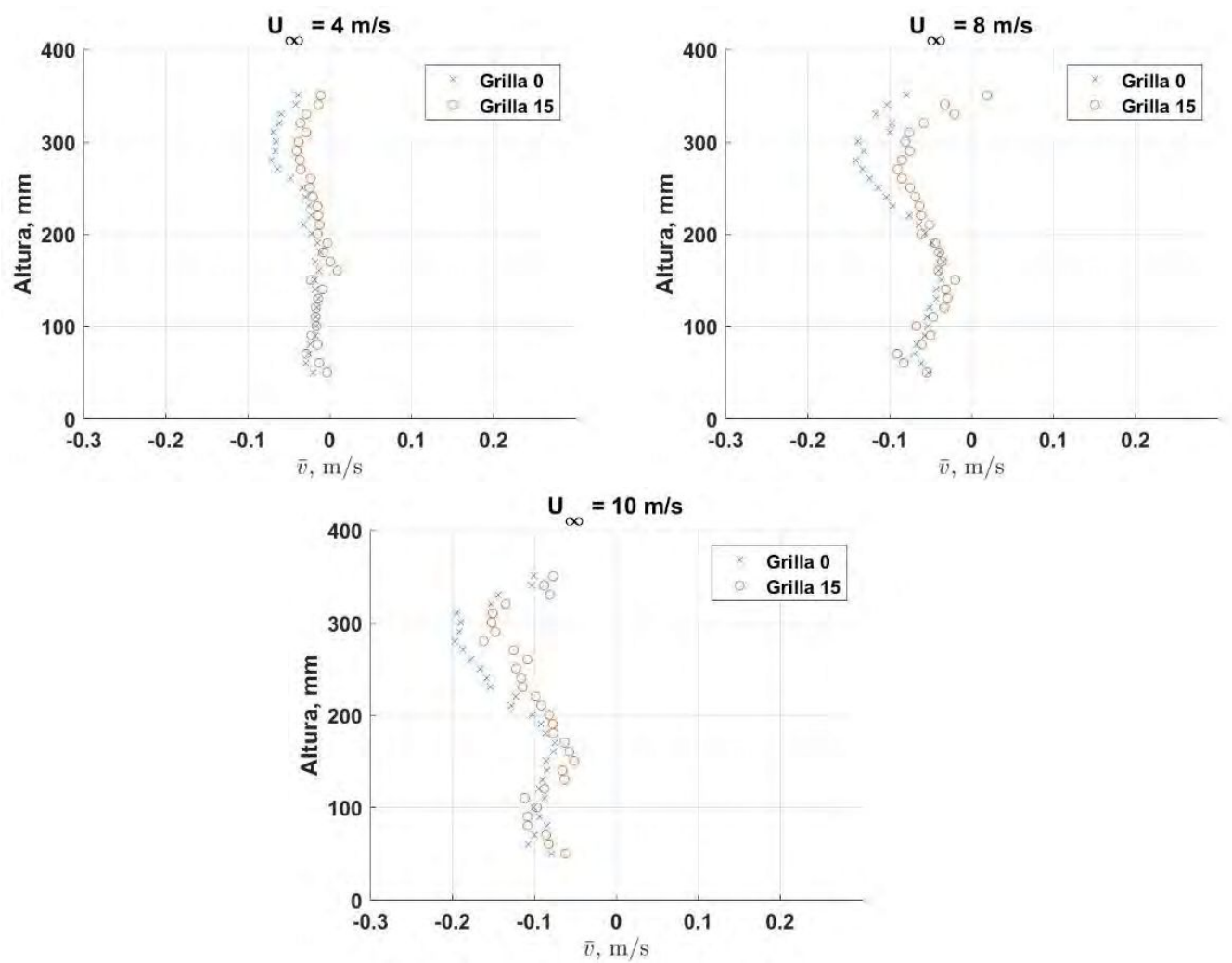

Figura 3.2. Velocidad media vertical para cada grilla en las tres velocidades utilizadas.

Si se analiza en detalle la componente angular de la velocidad, midiendo este ángulo como el arco tangente de la relación entre la velocidad vertical y la velocidad longitudinal $\left(\operatorname{atan}\left(\frac{\bar{v}}{\bar{u}}\right)\right)$, se puede encontrar que en la zona central de la sección de prueba del túnel, este ángulo varía entre $-0,2^{\circ}$ y $-0,65^{\circ}$ aproximadamente, para todos los casos analizados. Es de importancia conocer este ángulo dado que el ángulo de ataque, por definición, es el ángulo entre la cuerda del modelo con respecto a la velocidad, por ende, es necesario conocer la orientación del vector velocidad. Luego, en el desarrollo de esta tesis, cuando nos referenciemos al ángulo de ataque, será respecto a este vector velocidad, obtenido en estas mediciones, el cual depende de la configuración (con o sin grilla) y de la velocidad de prueba. En la Tabla 3.1 se puede ver un resumen del valor del ángulo de ataque medio, tomando la media como el promedio del ángulo de inclinación del vector velocidad en grados, una cuerda hacia arriba y una cuerda hacia abajo del centro de la sección de prueba, donde se emplazará el modelo.

Tabla 3.1. Ángulo medio del vector velocidad en la sección de prueba para las distintas configuraciones

\begin{tabular}{l|cc} 
Velocidad & Sin grilla & Con grilla \\
\hline $4 \mathrm{~m} / \mathrm{s}$ & $-0,36^{\circ}$ & $-0,21^{\circ}$ \\
$8 \mathrm{~m} / \mathrm{s}$ & $-0,52^{\circ}$ & $-0,35^{\circ}$ \\
$10 \mathrm{~m} / \mathrm{s}$ & $-0,72^{\circ}$ & $-0,52^{\circ}$
\end{tabular}

Luego, se analizaron las escalas espaciales integrales características del flujo. Para ello, se calcularon los valores de auto correlación de cada señal y utilizando el valor temporal para el cual la auto correlación toma el valor de 1/e y la velocidad de la corriente libre, se determinó la escala espacial (implementación de la técnica de flujo congelado). Con dicha metodología se graficaron los resultados, hallando que para el caso de flujo libre ("Grilla 0"), los valores en la escala longitudinal son dispersos en toda la sección, sin hallar un patrón definido, mientras que para la condición con grilla turbuladora ("Grilla 15") las escalas presentan un valor de $25 \mathrm{~mm}$ aproximadamente (25\% de la cuerda del perfil). Ver Figura 3.3. Al analizar la escala en el sentido vertical, ocurre algo similar, aunque la escala para el caso limpio se encuentra más definida, en torno a un valor de 
$30 \mathrm{~mm}$, mientras que la implementación de la grilla disminuye la escala turbulenta a un valor de $15 \mathrm{~mm}$, como se puede observar en la Figura 3.4. Este comportamiento se encuentra en todas las velocidades ensayadas. En las figuras se muestran solo los casos de mínima velocidad y máxima, 4 y $10 \mathrm{~m} / \mathrm{s}$ respectivamente, pero en 8 $\mathrm{m} / \mathrm{s}$ se encontraron los mismos resultados.
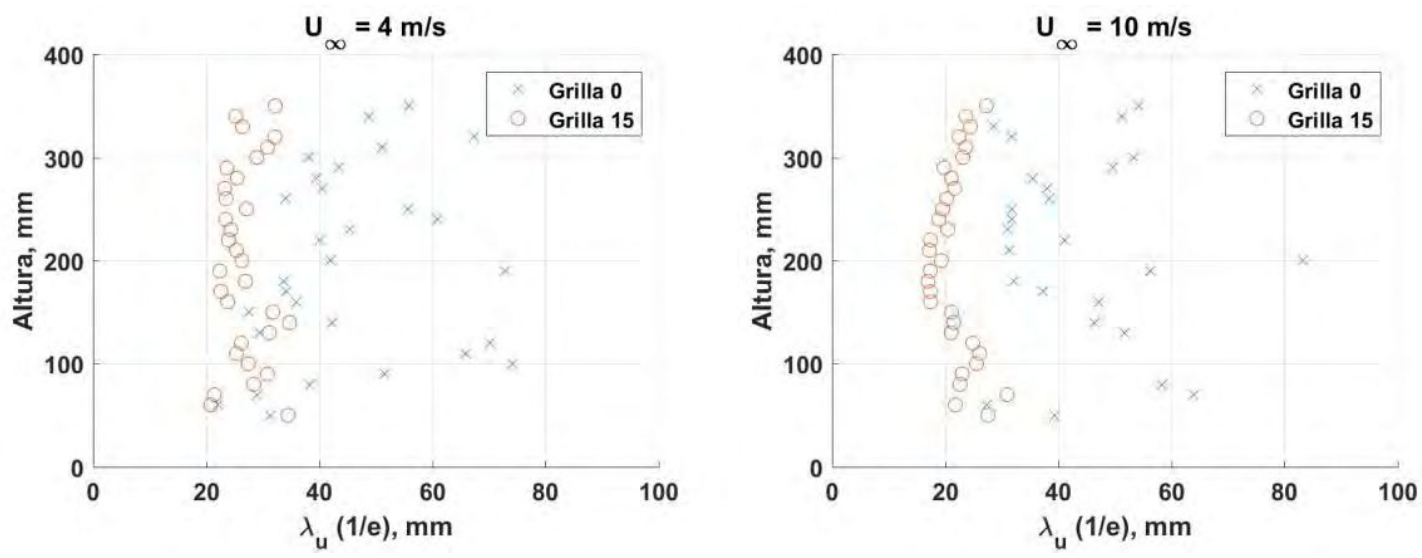

Figura 3.3. Escala integral espacial en la componente longitudinal. Usando criterio 1/e en la auto correlación.
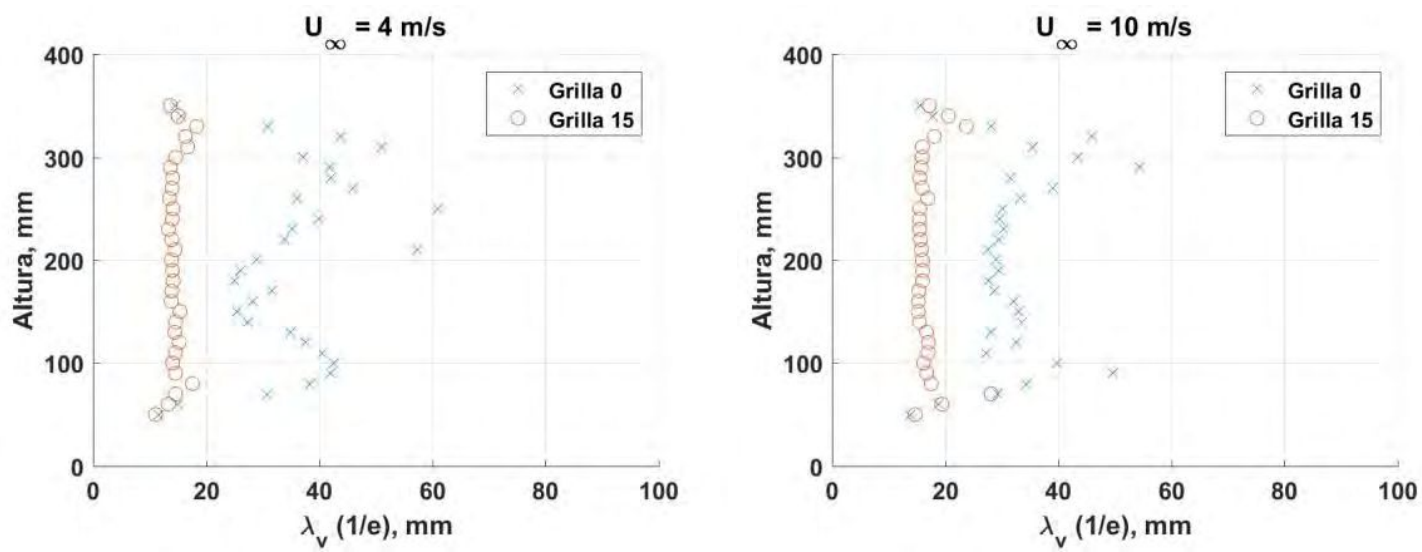

Figura 3.4. Escala espacial integral en la componente vertical de la velocidad. Usando criterio 1/e en la auto correlación.

Para completar el análisis de los niveles de turbulencia que se poseen en ambas condiciones, se calculó la intensidad de turbulencia, la cual nos brinda una magnitud que representa que desviación respecto de la media presenta la velocidad. En la componente longitudinal, ver Figura 3.5, se puede apreciar como la presencia de la grilla no genera un cambio importante en la intensidad de turbulencia, más allá de homogenizar esta característica. En cambio, para la componente vertical, ver Figura 3.6, la intensidad de turbulencia se duplica al implementar el sistema de grilla utilizado de $0,3 \%$ a $0,6 \%$ aproximadamente.

Por último, pero por eso no menos importante, se analizaron los valores del espectro de potencia de las señales de velocidad para la posición central de la zona de pruebas (lugar donde fueron emplazados los modelos, correspondiente con el eje de rotación). La Figura 3.7 muestra la energía normalizada para las tres velocidades de corriente libre, y se pueden encontrar algunas diferencias entre ellas. En un círculo gris, se muestra como para el cambio de grillas, en la velocidad de $4 \mathrm{~m} / \mathrm{s}$ se pueden encontrar diferencias en los niveles de energía presentes en los valores cercanos a $100 \mathrm{~Hz}$, mientras que para la velocidad de 8 y $10 \mathrm{~m} / \mathrm{s}$ dichas diferencias no son tan notorias. Una situación similar se puede observar para los espectros de potencia de la componente vertical de la velocidad, aunque en esta componente el aumento de energía se encuentra presente en las tres velocidades ensayadas. En el análisis a $4 \mathrm{~m} / \mathrm{s}$ se encuentran diferencias energéticas al modificar la turbulencia 
a partir de $10 \mathrm{~Hz}$, mientras que a 8 y $10 \mathrm{~m} / \mathrm{s}$ dichas diferencias se pueden apreciar recién para frecuencias mayores a $30 \mathrm{~Hz}$ aproximadamente.
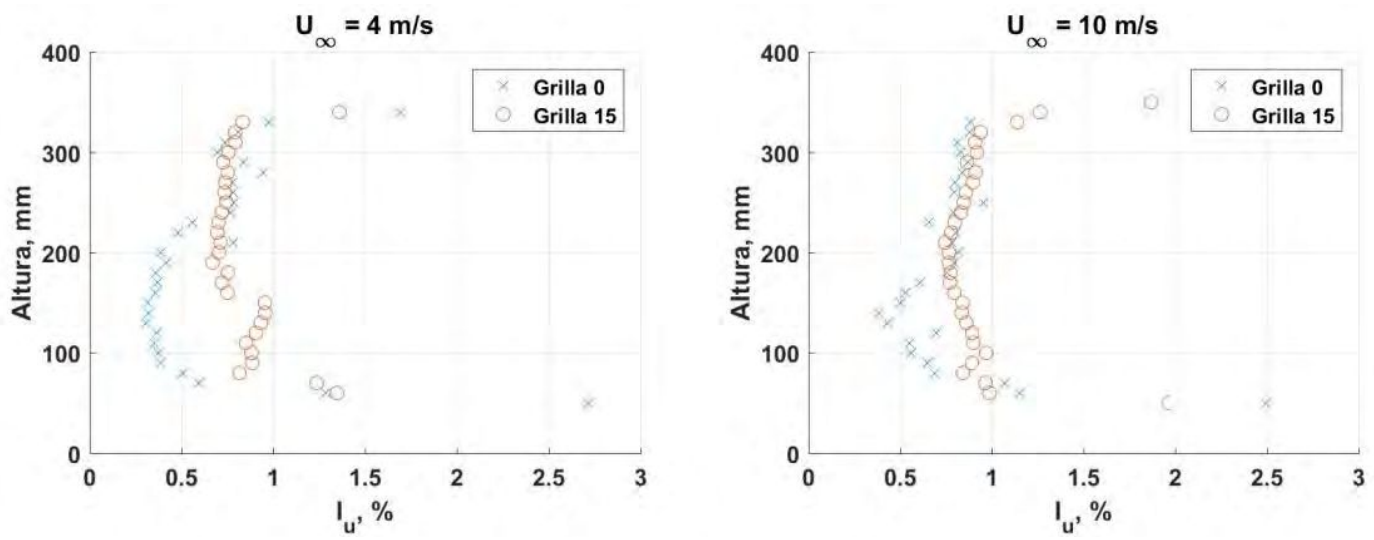

Figura 3.5. Intensidad de Turbulencia de la componente de velocidad longitudinal.
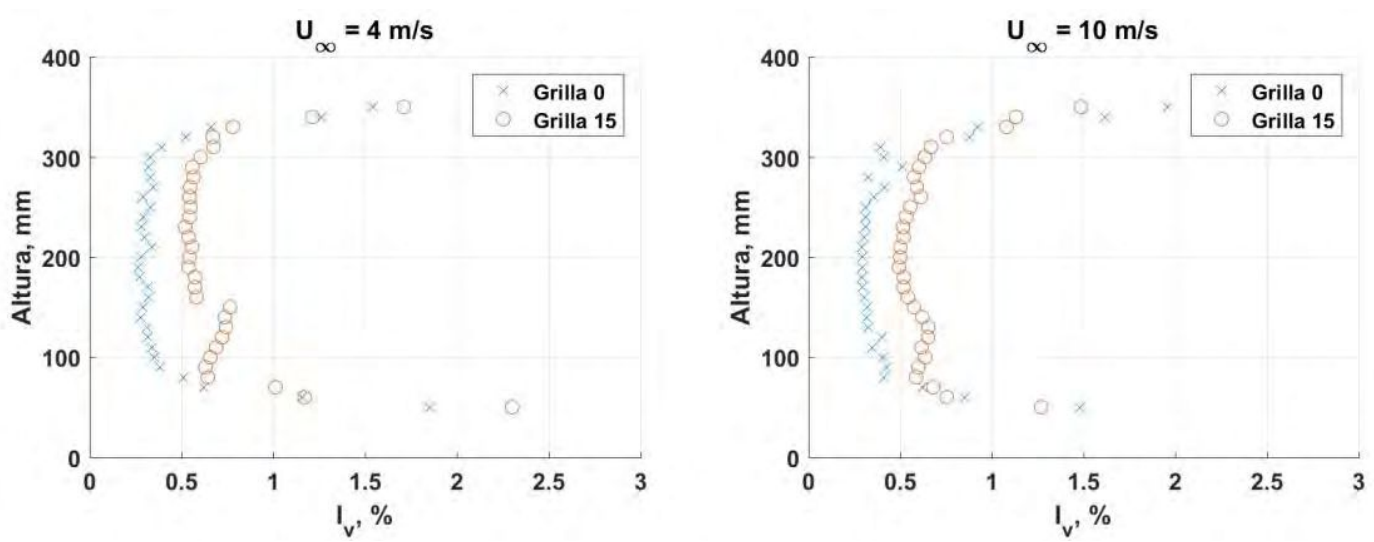

Figura 3.6. Intensidad de turbulencia de la componente de velocidad vertical. 

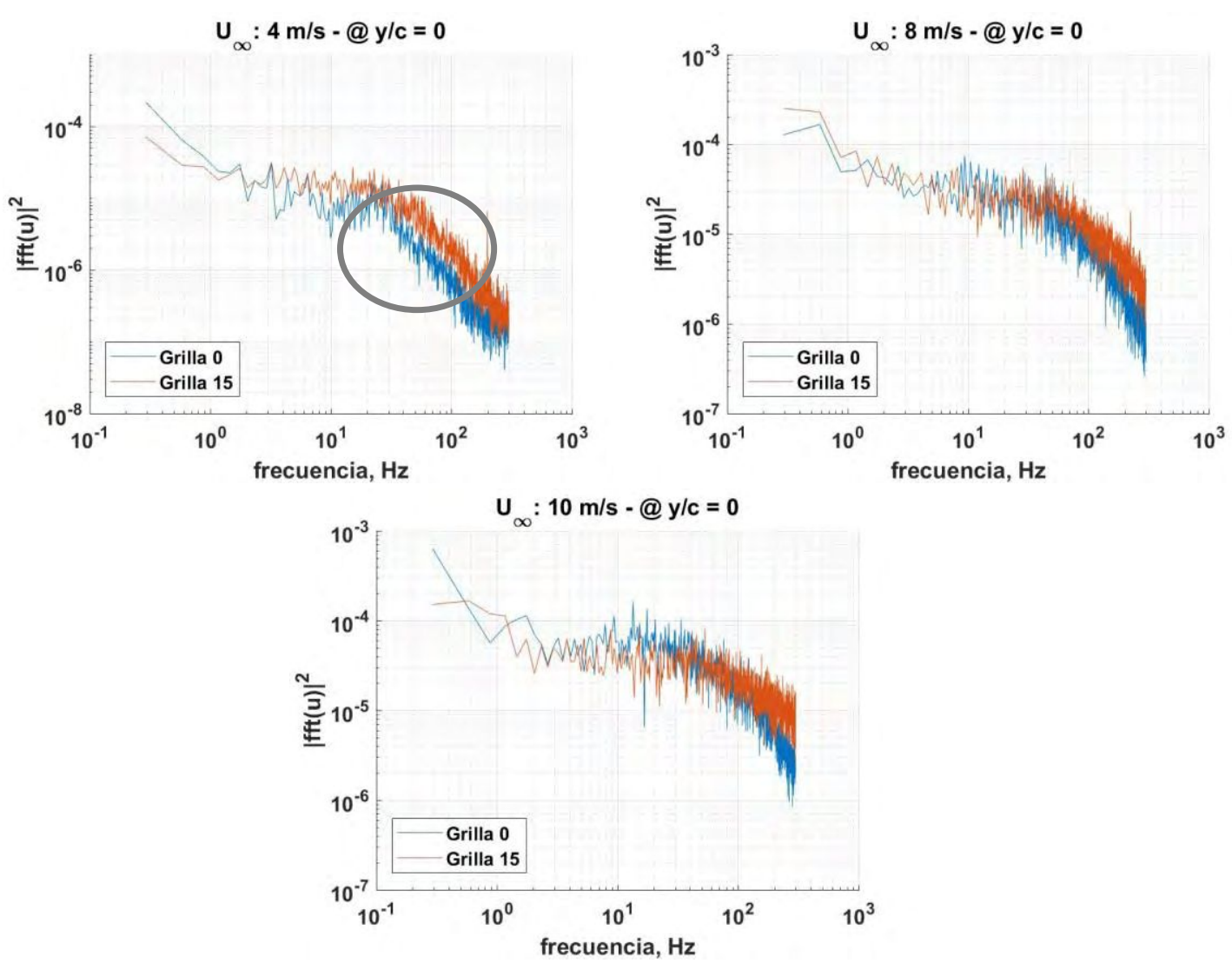

Figura 3.7. Espectro de energía de la componente longitudinal para las tres velocidades de referencia.
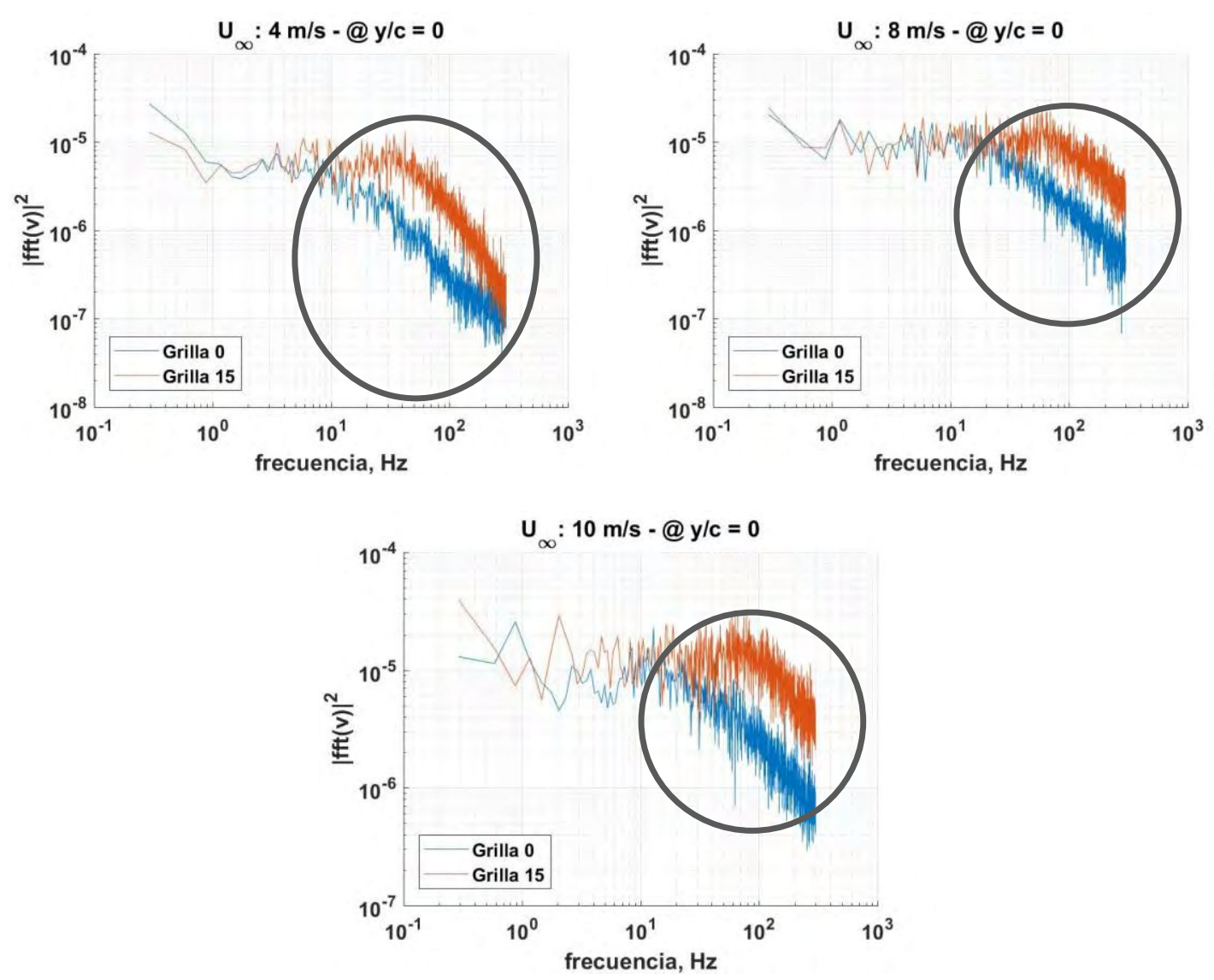

Figura 3.8. Espectro de energía de la componente vertical de la velocidad para las tres velocidades de referencia. 


\section{"Estudio aerodinámico experimental en flujo turbulento de bajo Reynolds sobre alas con movimiento de cabeceo"}

\subsection{Medición de fuerza aerodinámica}

\subsubsection{Movimiento tipo rampa: Caso validación}

Inicialmente, para poder analizar la metodología de ensayo, y utilizar un caso conocido en la bibliografía, se decidió realizar un estudio sobre un movimiento tipo rampa, similar al realizado en el trabajo AVT-202, y poder comparar los resultados hallados. Para ellos se realizaron los estudios sobre este movimiento, en velocidades de rotación similares, aunque un poco menores, y se comparan los resultados. A su vez, se realizó el análisis de influencia de la intensidad de turbulencia.

\subsubsection{Características del movimiento}

Para poder buscar efectos aerodinámicos no estacionarios, donde haya interacción de vórtices con distintos campos fluido dinámicos (turbulencias incidentes), se generó un movimiento de cabeceo puro (variación del ángulo de ataque) a distintas velocidades de rotación. Con la combinación de velocidad de rotación, cuerda y velocidad de la corriente libre se obtuvieron las siguientes frecuencias reducidas, que para movimientos transitorios se puede definir como:

$$
K=\frac{\dot{\Theta} c}{2 U_{\infty}}
$$

Siendo $U_{\infty}$ la velocidad de la corriente libre, c la cuerda del ala y $\dot{\theta}$ la velocidad de rotación. Utilizando las mismas velocidades de corriente libre previamente definidas $(4,8$ y $10 \mathrm{~m} / \mathrm{s})$ y teniendo en cuenta las limitaciones del sistema electromecánico, se pudieron representar frecuencias reducidas de 0,$025 ; 0,01$ y 0,005 para la velocidad de $4 \mathrm{~m} / \mathrm{s}$, mientras que para 8 y $10 \mathrm{~m} / \mathrm{s}$ se lograron las dos frecuencias reducidas menores. Para poder reducir la aceleración en los cambios de pendiente del movimiento rampa (comienzo y finalización del movimiento), se utilizó la expresión desarrollada por Eldredge, 2016 la cual suaviza dichos cambios, minimizando los picos de aceleración, lo que simplifica la medición de las fuerzas, dado que, si se minimiza la aceleración, se minimiza también los esfuerzos inerciales. A continuación, se grafican la evolución del ángulo de cabeceo $(\theta)$ utilizado en el caso de corriente incidente de $4 \mathrm{~m} / \mathrm{s}$.

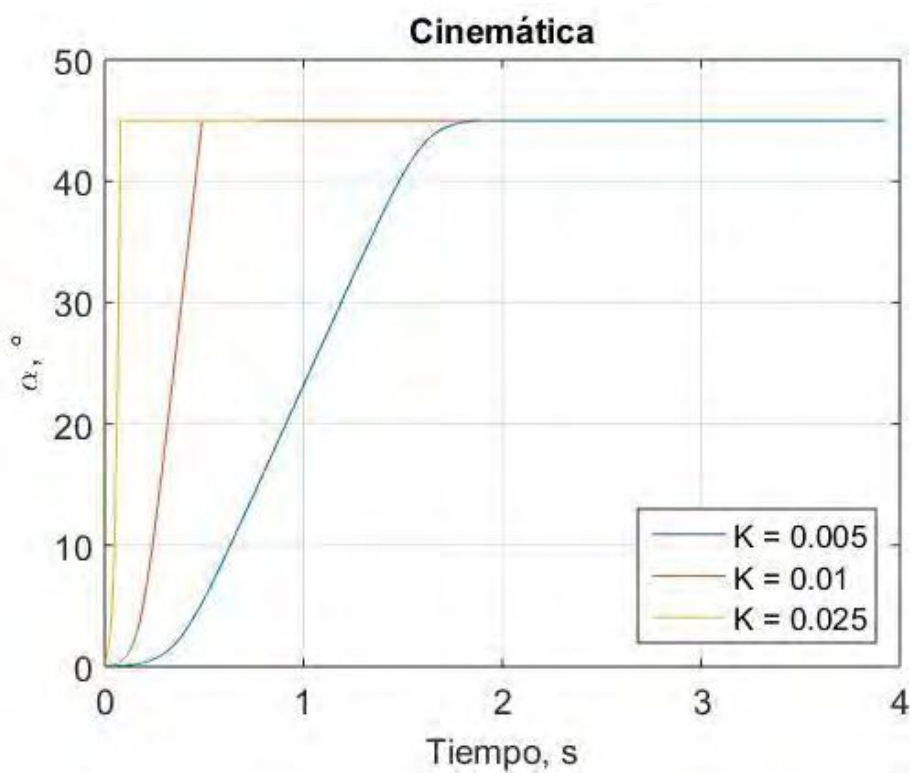

Figura 3.9. Distintas frecuencias reducidas analizadas para la condición de Reynolds 23000 con movimiento tipo rampa hasta $45^{\circ}$ de ángulo de ataque. 


\subsubsection{Estimaciones teóricas}

Antes de la realización del ensayo, se realizaron aproximaciones del coeficiente de sustentación que se debería medir para los distintos ensayos propuestos. Para realizar dicha aproximación se utilizó el modelo simplificado presentado por Babinsky et al. 2016 el cual simplifica la aerodinámica del fenómeno en estudio en cuatro contribuciones principales. Los resultados de la aplicación de dicho método se pueden observar en la Figura 3.10 .

Para los cálculos con el modelo empleado, se asumió que la velocidad relativa de desplazamiento entre el vórtice de borde de ataque (LEV) respecto al desprendido en borde de fuga (TEV) es de 0,2 veces la velocidad de la corriente libre. Dicho valor es un poco menor al encontrado experimentalmente con técnicas de PIV (un tercio a mitad de la velocidad de la corriente libre) para casos de rotación con velocidades mayores. El valor utilizado de 0,2 fue elegido para que el coeficiente luego de la sección transitoria coincida con el valor del coeficiente de sustentación hallado en los ensayos estáticos.

De los valores teóricos obtenidos, se puede observar como el caso de frecuencia reducida 0,005 no genera un aumento de sustentación apreciable durante el movimiento del ala, con lo cual deberían coincidir con los valores estáticos del ala (movimiento cuasi-estático). En la frecuencia 0,01 ya se empiezan a apreciar efectos dinámicos o no-estacionarios generando un incremento de la sustentación sobre todo cerca del momento en que el ala está llegando a su momento de reposo. Al considerar el caso de 0,025 , ya los efectos dinámicos son muy considerables, obteniendo variaciones a lo largo de todo el trayecto de rotación del ala.

Con los tres escenarios elegidos, se pueden observar comportamientos totalmente diferentes para analizar si la turbulencia realiza modificaciones en los patrones de flujo para casos cuasi-estáticos, y completamente dinámicos. 

de cabeceo"

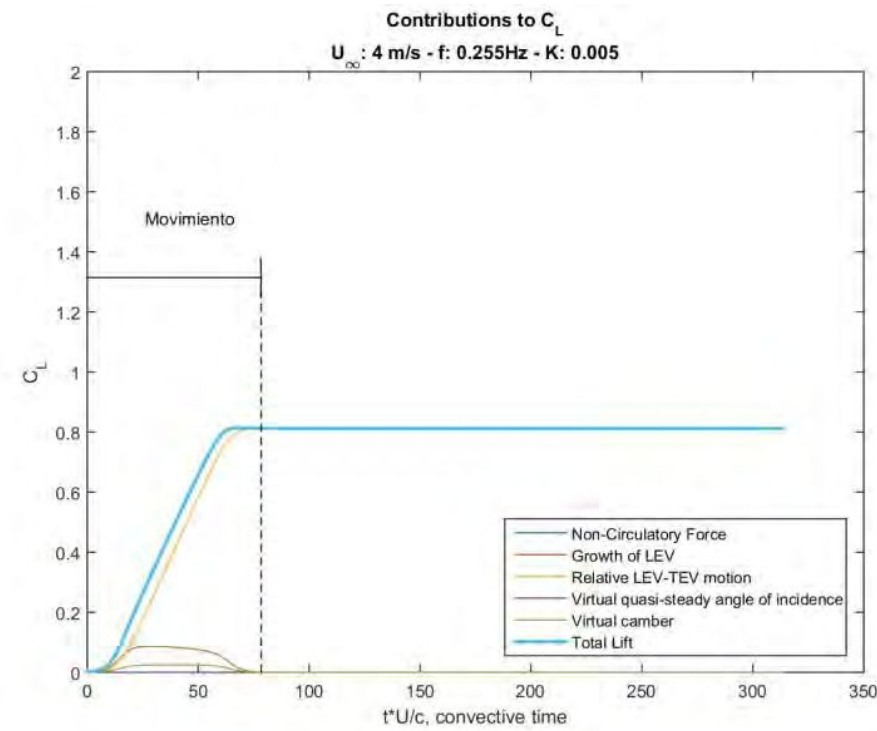

Contributions to $\mathrm{C}$

$U_{\infty}: 4 \mathrm{~m} / \mathrm{s} \cdot \mathrm{f}: 0.51 \mathrm{~Hz} \cdot \mathrm{K}: 0.01$

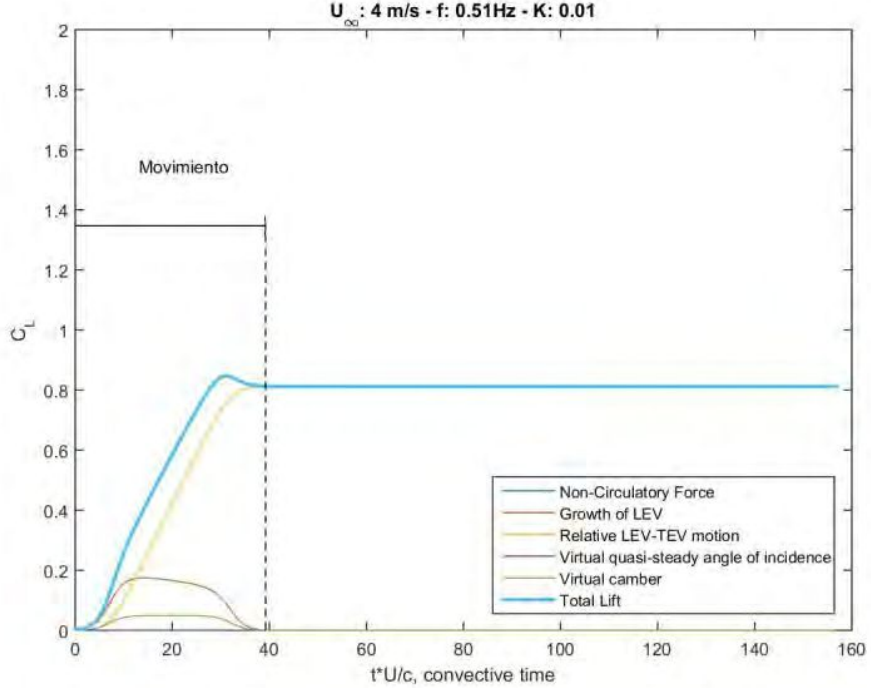

Contributions to $C_{1}$

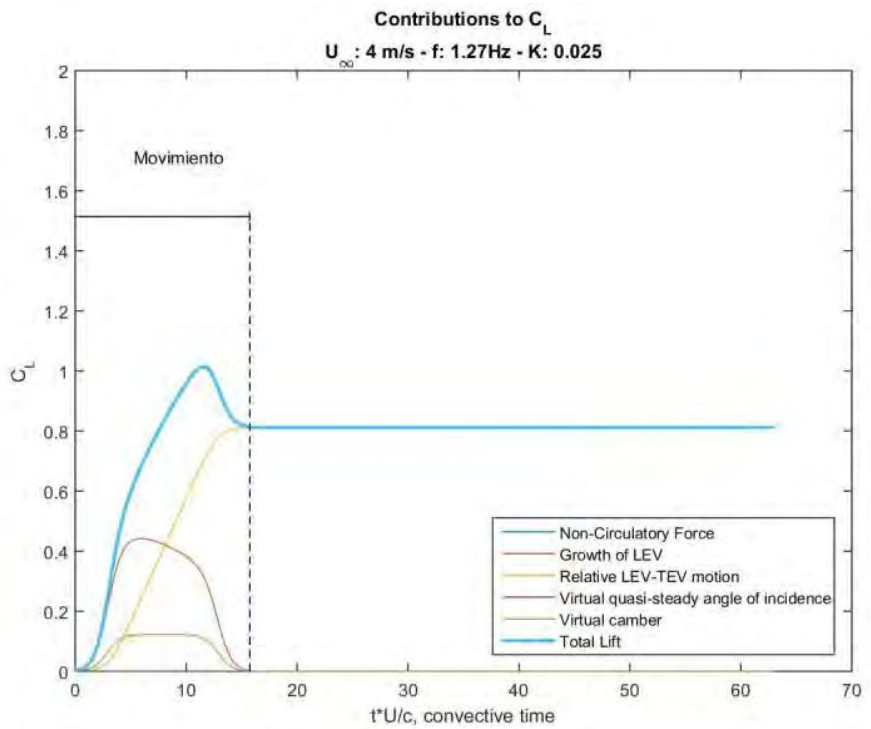

Figura 3.10. Coeficiente de sustentación teórico para tres valores diferentes de frecuencia reducida al mismo número de Reynolds (23000). 


\section{“Resultados y Análisis”}

\subsubsection{Cargas Aerodinámicas}

\subsection{Cargas a mínima intensidad de turbulencia}

Se realizaron distintas repeticiones del ala rotando con distintas velocidades de corriente libre para analizar la evolución de la fuerza aerodinámica en los casos dinámicos a distintos números de Reynolds.

Al comparar la cinemática del ala, se puede ver que en todos los casos hay muy buena repetitividad en los movimientos. La cinemática enviada como comando es replicada por el ala, sin haber demoras y sin errores considerables. Ver Figura 3.11. A su vez, si comparamos los resultados de la medición realizada con el sensor angular y el acelerómetro, se puede ver como el acelerómetro es errático debido a las aceleraciones generadas en la rotación del ala. Para poder considerar dicha medición correcta hay que corregir dicha señal por la aceleración generada con el movimiento. Pero, se puede utilizar el acelerómetro para medir en condiciones estáticas, mientras que el sensor angular, sirve para mediciones dinámicas.

Otro punto por destacar es la comparación entre las distintas repeticiones, el movimiento realizado es siempre el mismo, lo cual es bueno para poder lograr una buena repetitividad en los ensayos, así como encontrar tendencias que se repitan y no sean un caso particular. Dicho análisis puede ser observado en el Análisis de repetitividad de cargas aerodinámicas.

Dados estos resultados, lo importante para esta tesis es la medición de la fuerza aerodinámica, eliminando la componente inercial en la medición. Como se puede observar en el Validación del método de tarado dinámico, el elemento equivalente es el mejor método para caracterizar las fuerzas inerciales producto de la rotación de un elemento.

En primera instancia, como se observa que las mediciones realizadas tienen una componente de alta frecuencia, estilo ruido, primero se le pasó un filtro digital pasa bajo de forma Chebyshev tipo II con frecuencia de pasa banda hasta $20 \mathrm{~Hz}$ y una frecuencia de corte en $25 \mathrm{~Hz}$ con una atenuación de $20 \mathrm{~dB}$. Con eso pudimos eliminar el ruido en las señales y quedarnos con la parte más importante, aunque todavía queda la oscilación de $10 \mathrm{~Hz}$ producto de las vibraciones del modelo. Luego se aplicó en la señal otro filtro Chebyshev Tipo II, pero con frecuencia de pasa banda $9 \mathrm{~Hz}$ y frecuencia de corte en $11 \mathrm{~Hz}$ con atenuación de $40 \mathrm{~dB}$, para eliminar las vibraciones mecánicas. Las respuestas en magnitud de ambos filtros se pueden ver en la Figura 3.12.

Además, se generaron gráficas que comparan los resultados experimentales obtenidos, con los resultados teóricos planteados en la sección anterior de este capítulo, así como también respecto a los valores de la fuerza cuasi-estática. Dichos resultados se pueden analizar en la Figura 3.13. Como se puede observar en las mismas, para los casos dinámicos, si bien el gran salto de fuerza (coeficiente) se produce al finalizar el movimiento planteado y aparece un decaimiento que tiende al valor de la fuerza en condiciones estáticas, el coeficiente siempre tiene un valor mayor, del orden del $10 \%$

\subsection{Comparación de resultados dinámicos con otros autores}

Como forma de validación de los resultados hallados, se compararon los resultados con los expuestos en Ol y Babinsky, 2016. En dicho trabajo se muestran los gráficos de los resultados ante una rotación lenta (6 tiempos convectivos de duración del movimiento, $\mathrm{K}=0,065)$ para un caso de traslación y otro rotacional. En nuestros ensayos, el movimiento más rápido $(\mathrm{K}=0,025)$ representa una rotación en 16 tiempos convectivos. Se define tiempo convectivo al tiempo adimensionalizado con la cuerda del modelo ensayado y la velocidad de la corriente libre, representando el valor de un tiempo convectivo el tiempo en el que tarda una partícula de fluido en recorrer una distancia equivalente a una cuerda. 

de cabeceo"

$\mathrm{U}: 4 \mathrm{~m} / \mathrm{s}-\mathrm{K}: 0.025$
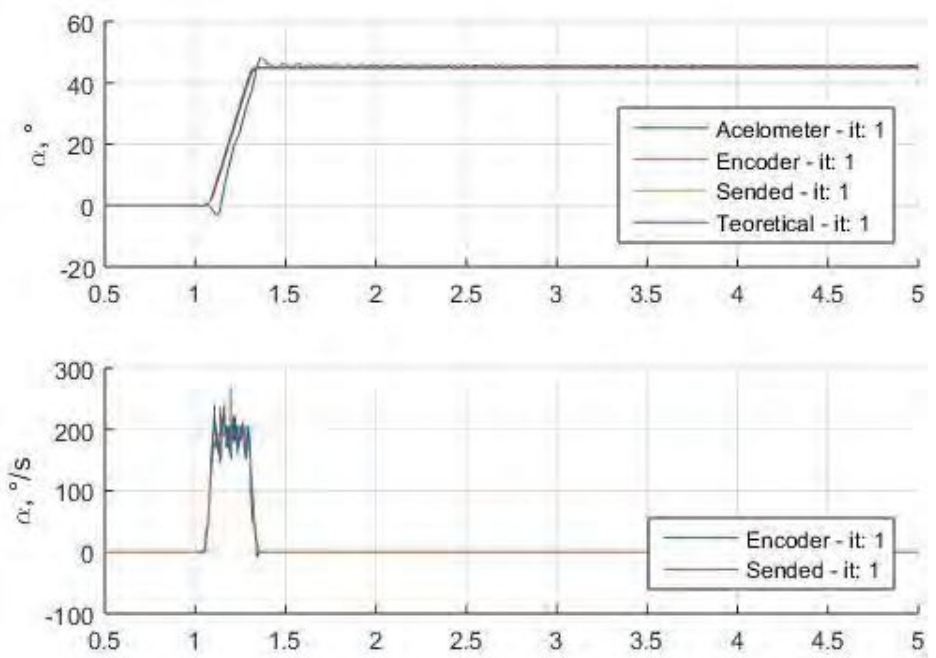

$\mathrm{U}: 10 \mathrm{~m} / \mathrm{s}-\mathrm{K}: 0.01$
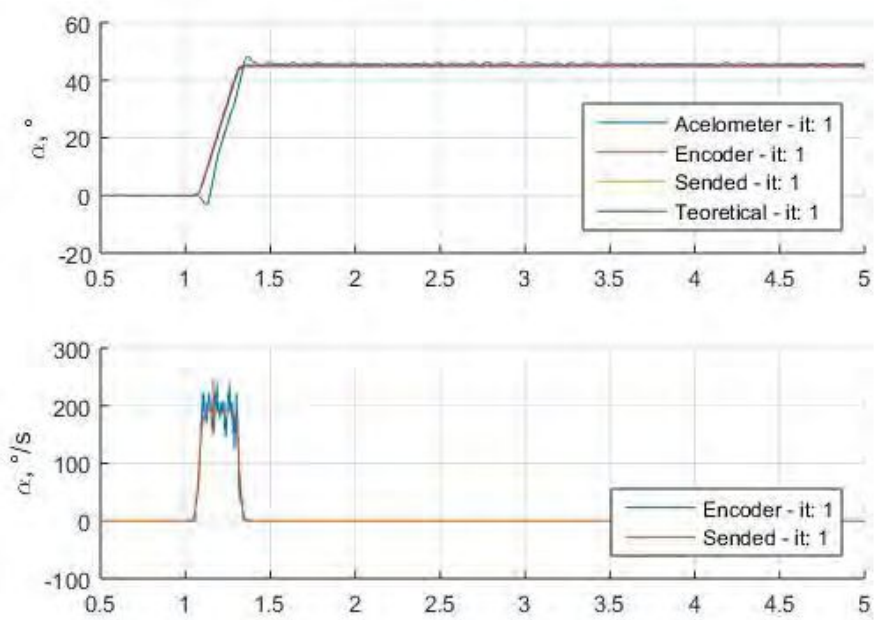

Figura 3.11. Comparación entre cinemática realizada por el ala y la enviada

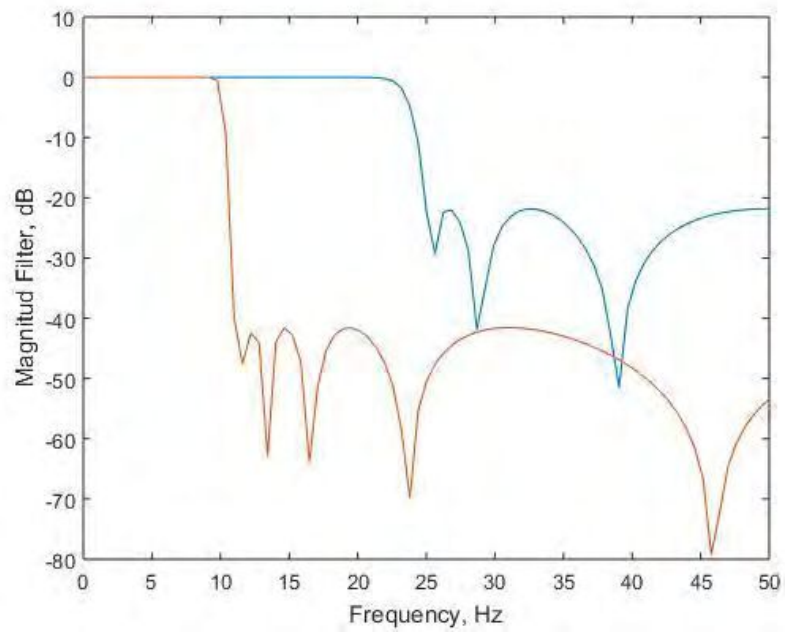

Figura 3.12. Respuesta de los filtros digitales implementados 


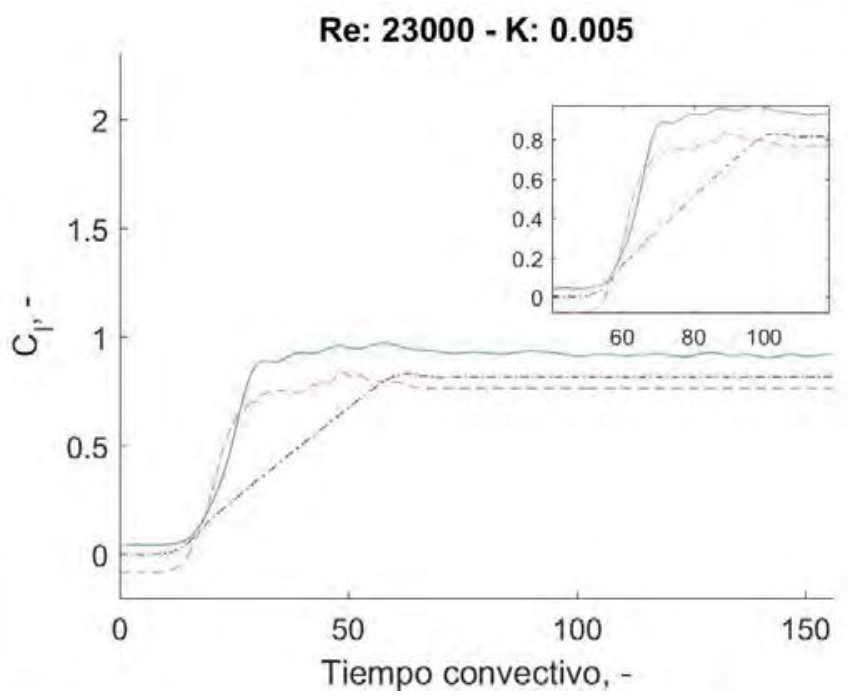

Re: 23000 - K: 0.01

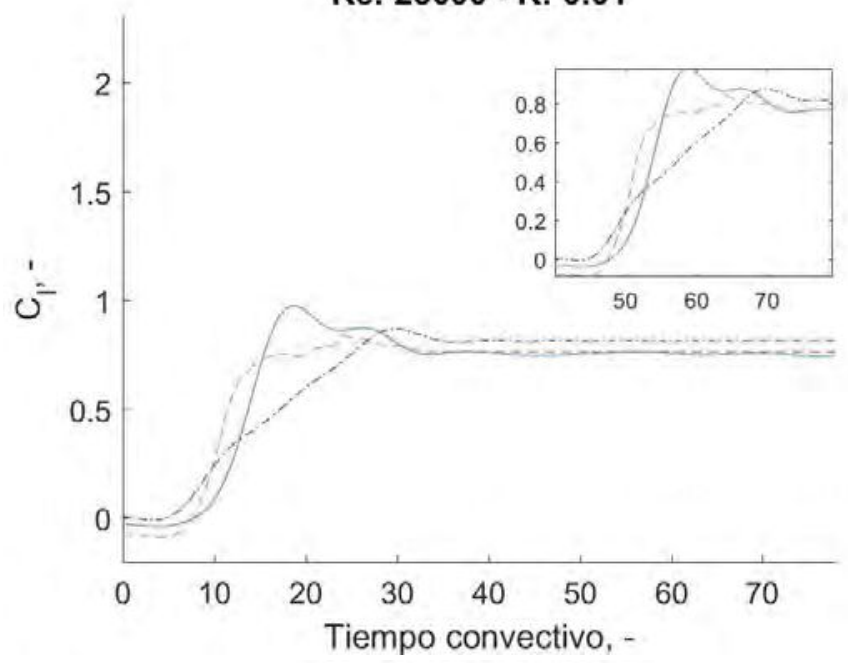

Re: 23000 - K: 0.025

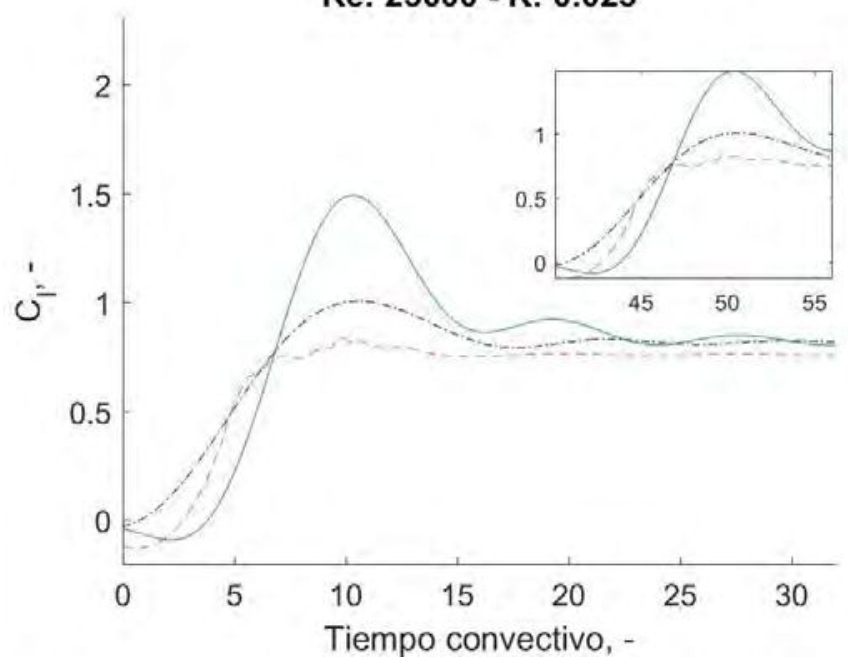

Figura 3.13. Media experimental (azul), estimación teórica (negro punteado) y valor cuasi estático (rojo punteado)

Respecto a los tipos de movimiento que ensayaron, en dicho trabajo los resume con la siguiente figura: 


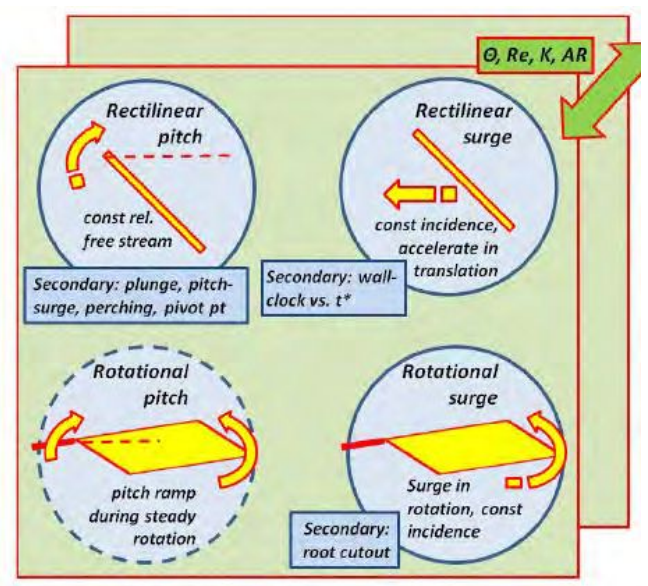

Figura 3.14. Tipos de movimientos ensayados en la referencia Ol y Babinsky 2016

Por lo tanto, comparado con el movimiento empleado en nuestro trabajo, nos debemos comparar con el movimiento "Rectilinear Pitch". Al ver los resultados expuestos para el coeficiente de sustentación en su caso "lento" se puede observar que el valor máximo de $C_{L}$ hallado es de un valor entre 1,2 y 1,5. Dicho pico se encuentra unos instantes previos a la finalización del movimiento y luego comienza un decrecimiento del coeficiente de sustentación que tiende al valor en estacionario. Cabe destacar que el resultado expuesto en la Figura 3.15 es para rotaciones respecto al borde de ataque, mientras que en nuestros ensayos las rotaciones son en torno a la mitad de la cuerda.

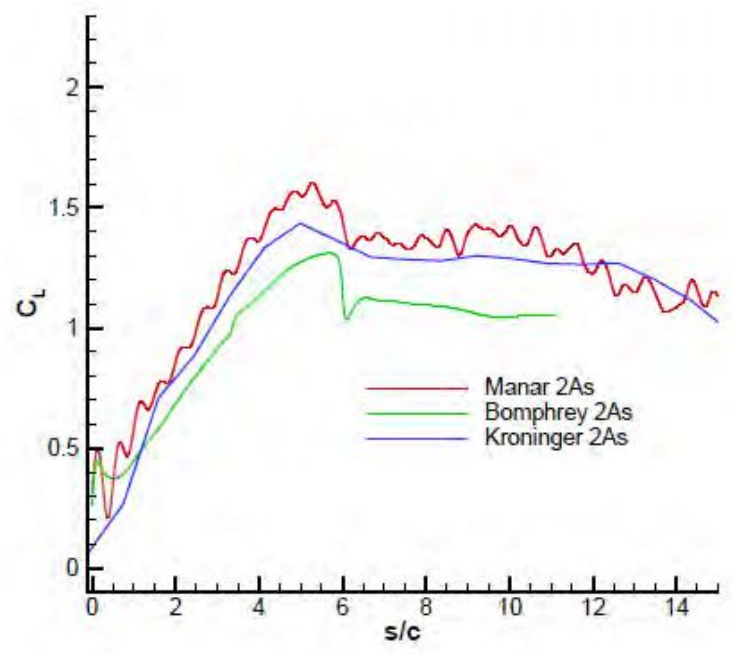

Figura 3.15. Resultados expuestos por M. Ol y H. Babinsky 2016 para un movimiento puro de cabeceo.

$\mathrm{Al}$ analizar el efecto del punto de pívot, se puede observar la Figura 3.16, donde demuestra los efectos de retardo que se producen en la sustentación, así como un decrecimiento en el pico de sustentación máximo cuando se retrasa el punto de rotación del ala. 

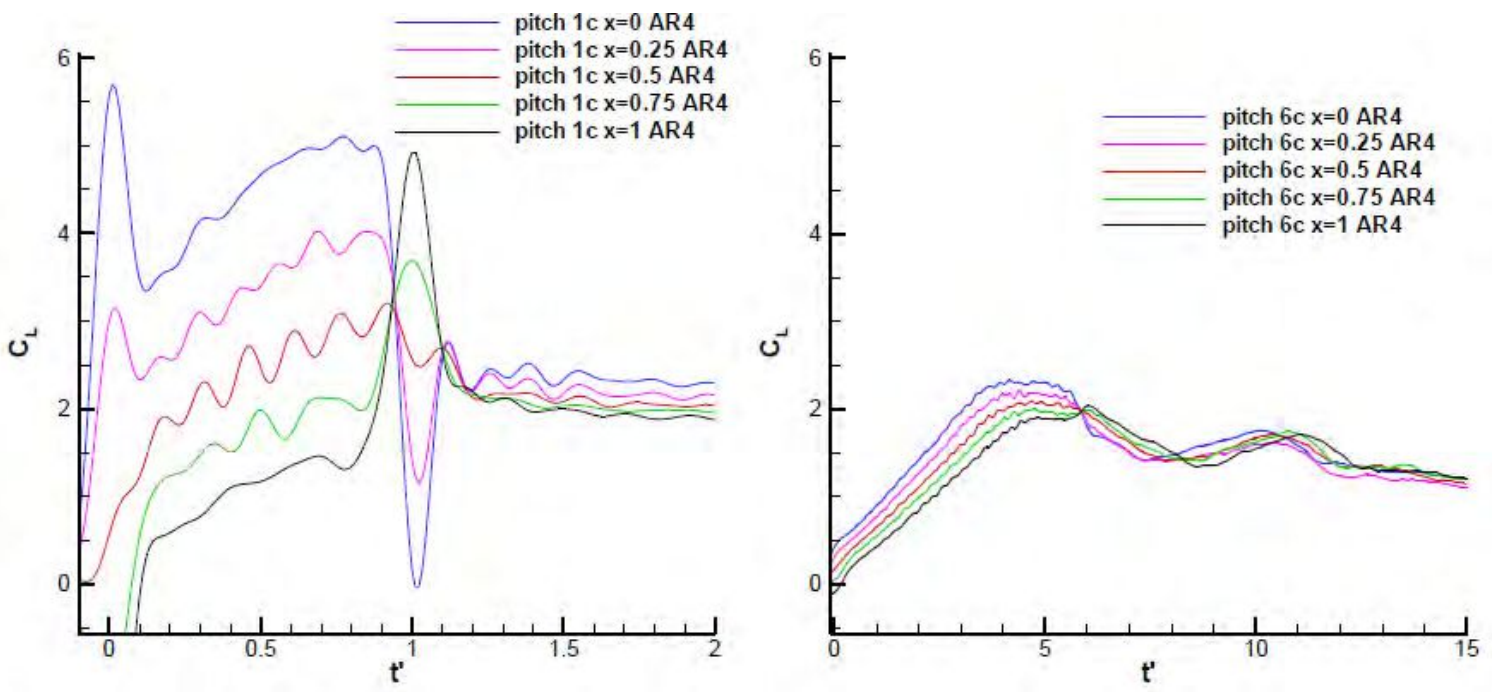

Figura 3.16. Efecto del punto de pívot. Referencia Ol y Babinsky 2016.

Todos los resultados extraídos del trabajo, mostrados con anterioridad, corresponden a diferentes números de Reynolds dado que son comparaciones de diferentes autores y distintos túneles de viento y/o túneles y tanques de agua.

Si comparamos los resultados de la referencia, con distinta frecuencia reducida pero también considerado un movimiento lento, podemos observar que en líneas generales el resultado es similar. Si bien, además, poseemos un nivel de turbulencia distintos (laminar en la referencia, respecto a $\sim 0,5 \%$ de intensidad de turbulencia), las magnitudes del pico son similares, y la duración parece similar (doble de tiempos convectivos del movimiento de rotación para llegar al valor estacionario). En la Figura 3.17 se pueden ver lado a lado los resultados de la referencia declarada, junto con el caso de mayor velocidad de rotación generado en este trabajo.
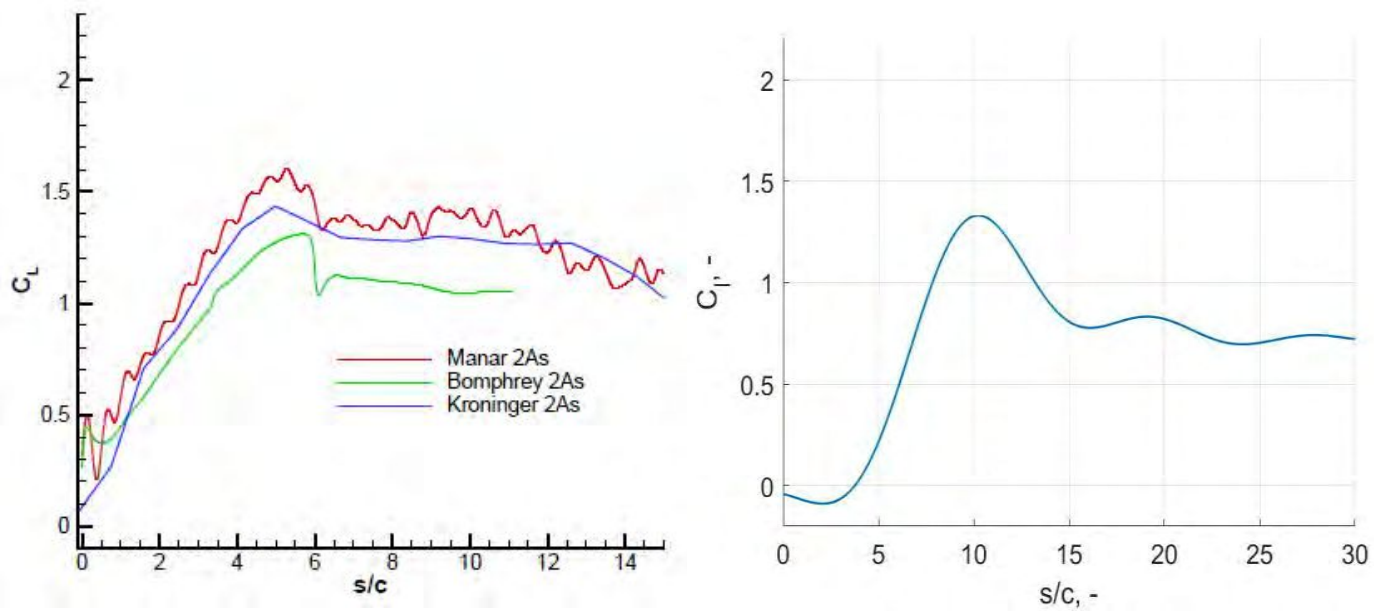

Figura 3.17. Comparación de resultados dinámicos con referencia bibliográfica. Izquierda movimiento que dura 6 tiempos convectivos (s/c) (Ol y Babinsky 2016), derecha movimiento que dura 16 tiempos convectivos.

\subsection{Ensayos con máxima turbulencia (I=1\%)}

Luego de haber analizado los resultados encontrados en los ensayos estáticos y dinámicos, se realizaron los ensayos dinámicos con las mismas frecuencias reducidas y mismos ángulos de ataque final de movimiento, pero con la turbulencia mayor, para poder comparar los resultados respecto a los ensayos con mínima turbulencia. El método de tarado es nuevamente el método del elemento equivalente dado que es el método que 
elimina todos los esfuerzos inerciales de la medición. Al igual que con la mínima turbulencia, se repitió 10 veces cada ensayo. Los resultados mostrados en este caso ya corresponden a los resultados luego de haber sido filtrados con los mismos filtros digitales especificados anteriormente.
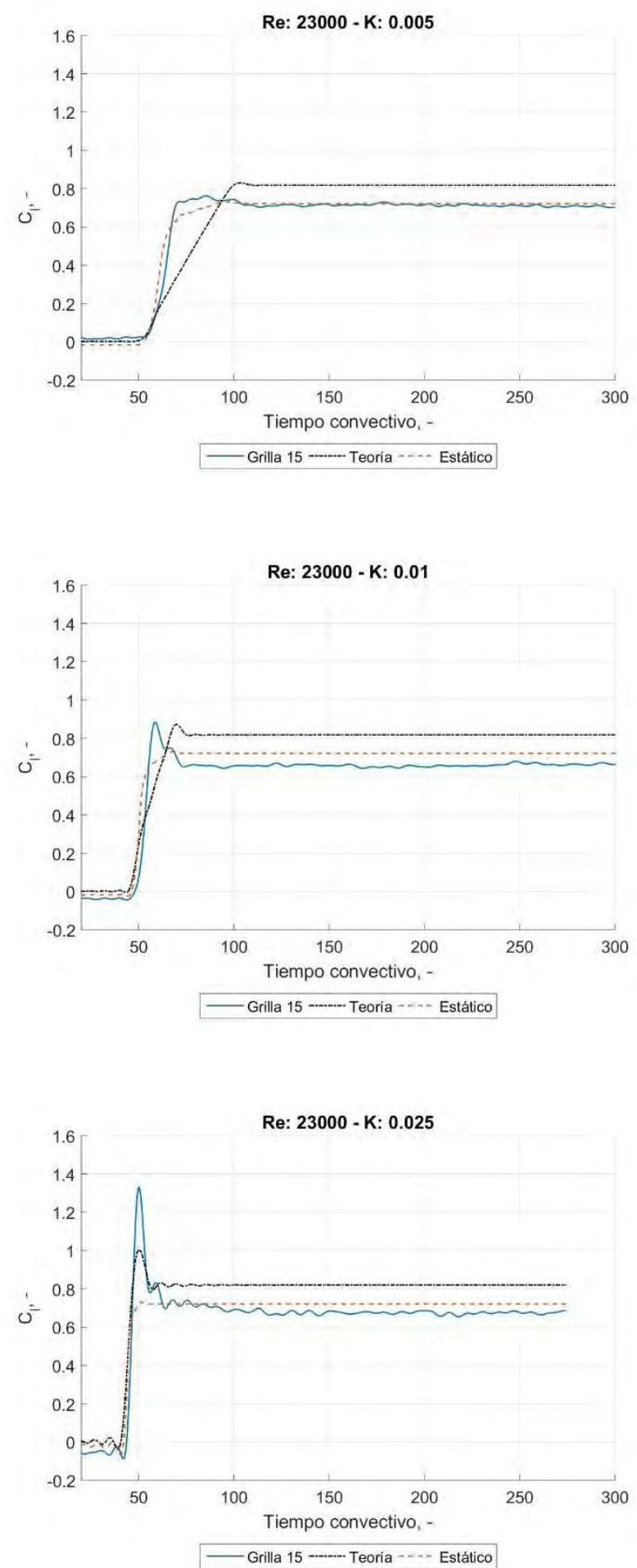

Figura 3.18. Media de los casos dinámicos a distintos números de Reynolds y distintas frecuencias reducidas. 


\subsection{Comparación del efecto de la turbulencia}

A continuación, se muestra la comparación de los resultados obtenidos con los ensayos dinámicos para los distintos niveles de turbulencia. A su vez se grafican los resultados teóricos esperados según el método implementado, como también una interpolación de los resultados estáticos (con mínima turbulencia) en función del ángulo de ataque que posee el ala instante a instante (método "cuasi estático").

En los resultados hallados, ver Figura 3.19, se puede observar un comportamiento similar al encontrado para el caso estático. Es decir, la turbulencia toma un rol importante en las cargas aerodinámicas generadas por la placa plana para bajos números de Reynolds, pero no cuando el Reynolds aumenta. Para el Reynolds de 23000, se pueden observar diferencias importantes entre el valor máximo encontrado en el coeficiente de sustentación para cada turbulencia ensayada (diferencias en torno al 13\% para la máxima frecuencia reducida ensayada). Sin embargo, al aumentar el número de Reynolds, estas diferencias se mitigan. 
"Estudio aerodinámico experimental en flujo turbulento de bajo Reynolds sobre alas con movimiento de cabeceo"
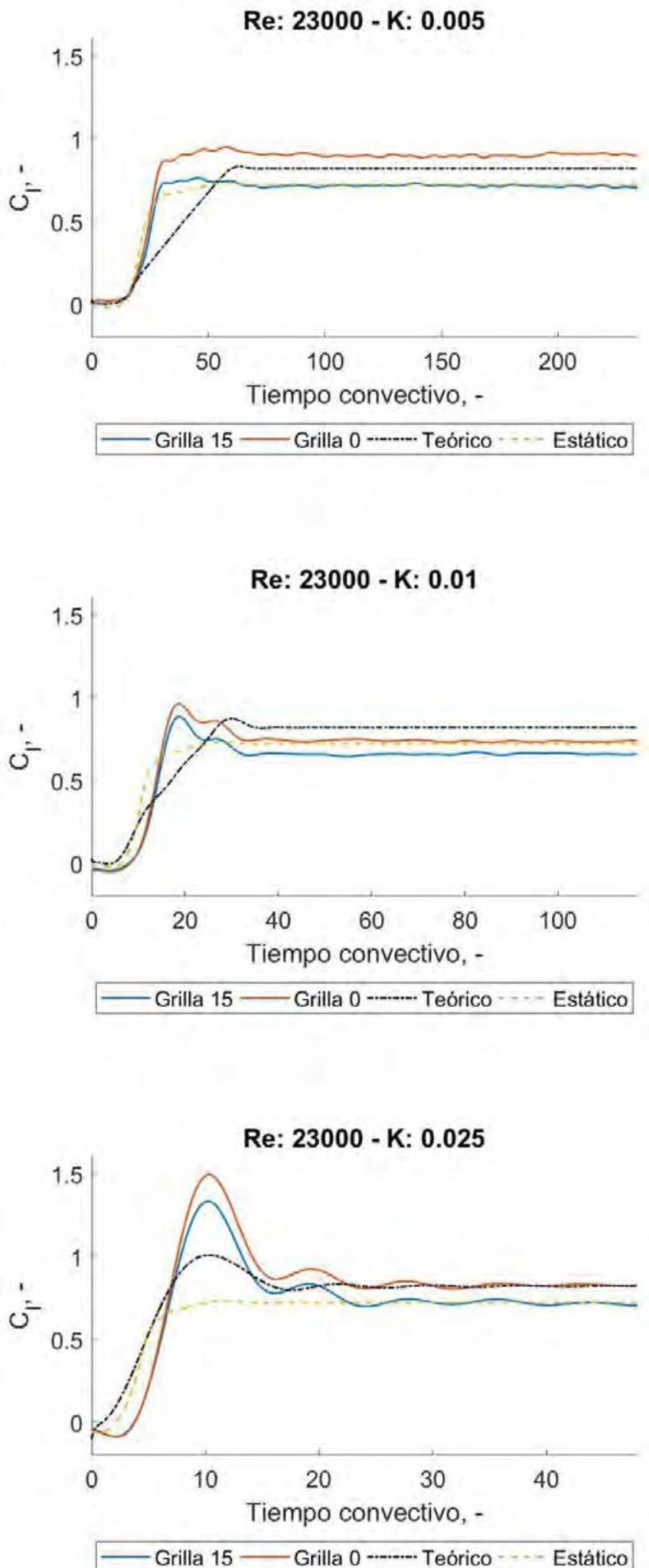

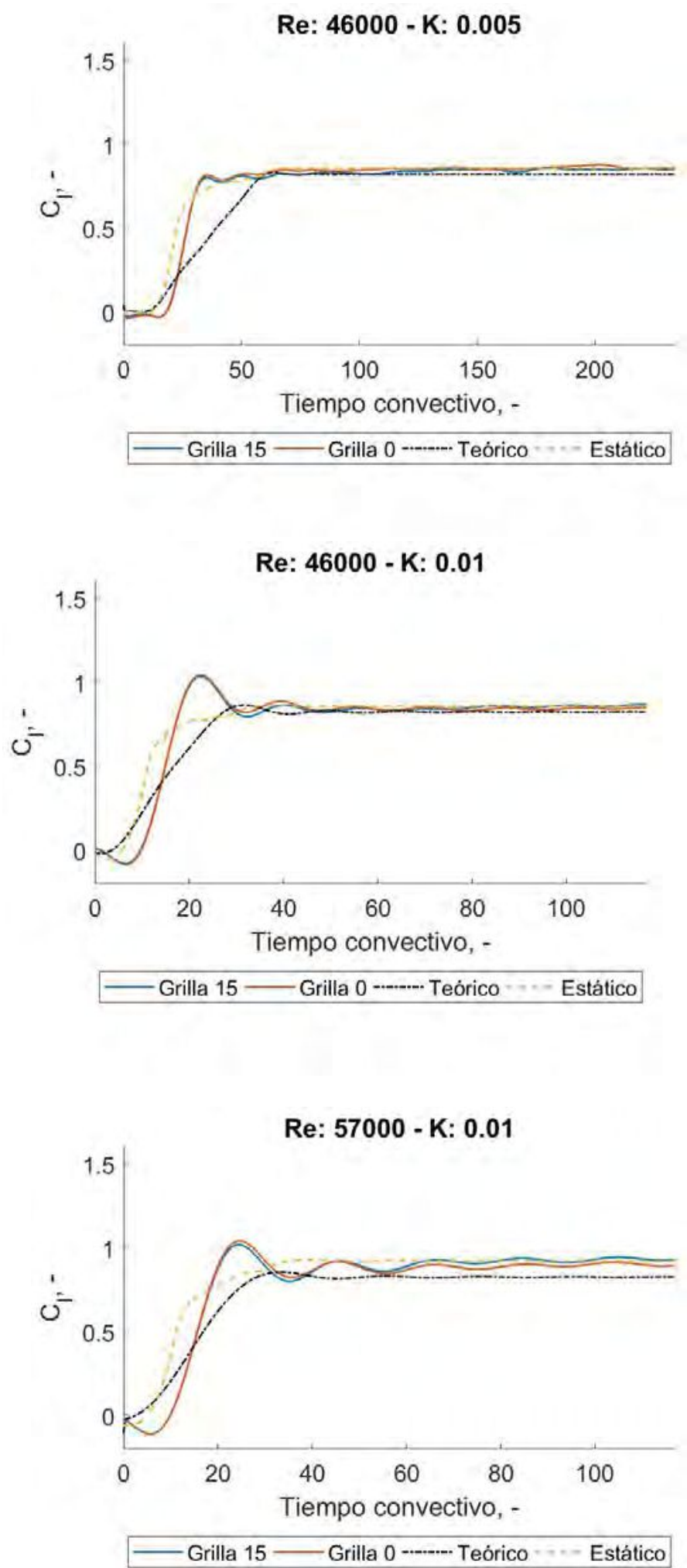

Figura 3.19. Comparación de resultados entre distintas turbulencias para distintos números de Reynolds y frecuencias reducidas. 


\section{"Estudio aerodinámico experimental en flujo turbulento de bajo Reynolds sobre alas con movimiento de cabeceo"}

\subsubsection{Visualizaciones}

\subsection{Condiciones Estáticas}

En primera instancia, se mostrarán los resultados obtenidos para ángulos de ataque fijos, o conocido como condición estacionaria. Allí se puede visualizar cuál es el comportamiento del flujo si no se modifica la condición de ángulo de ataque. Si bien, para grandes ángulos de ataque, en donde el perfil (placa plana), dado por el gradiente adverso de presiones, genera el desprendimiento de la capa límite, produce efectos no estacionarios sobre el flujo (desprendimientos vorticosos) se considera una condición estacionaria dado que el ala se encuentra fija en una posición.

En la Figura 3.20 se muestra una secuencia de imágenes con su configuración de flujo para distintos ángulos de ataque. En estas imágenes, se han localizado con círculos azules las zonas más importantes a destacar del flujo. Al ver que es lo que realiza el flujo de aire con el ala en $0^{\circ}$ de ángulo de ataque, se observa que hay flujo pegado a la superficie del ala todo el tiempo, como era de esperar para una placa plana sin ángulo de ataque. Cuando incrementamos el ángulo hasta cuatro grados, se puede ver una desviación del flujo desde el borde de ataque, pero todavía flujo pegado a toda la superficie, al incrementar el ángulo hasta $10^{\circ}$, se observa que el comportamiento medio del flujo es el de estar pegado hasta un porcentaje de la cuerda, y luego completamente separado. El comportamiento dinámico de lo que está aconteciendo en este ángulo de ataque se detallará en el siguiente párrafo. Al observar el comportamiento del flujo a un valor aún mayor, $15^{\circ}$, se observa flujo completamente despegado desde el borde de ataque, generado una estela muy turbulenta de un espesor del orden de la superficie proyectada en la vertical del ala. Si seguimos aumentando el ángulo de ataque, se puede observar como comienzan a aparecer desprendimientos vorticosos tanto desde el borde de ataque como el del borde de fuga, generando una fluctuación muy importante en el campo de flujo. Estos resultados se condicen con lo encontrado en la curva de sustentación en función del ángulo de ataque que se puede observar en la sección "3.2.1.1 Ensayos estáticos".

Anteriormente se mencionó que para el valor de $10^{\circ}$ de ángulo de ataque, se había observado un comportamiento dinámico sobre la superficie del ala. En la Figura 3.21 se puede observar como para un instante inicial, la capa límite se encuentra separada de la superficie superior del ala, mientras que, al ver los instantes temporales seguidos, debido al desprendimiento de un vórtice, se genera un pegado de la capa límite, pero por un tiempo muy limitado. Este fenómeno se repite periódicamente, aunque no siempre con la misma separación temporal. Si observamos lo que pasa con las cargas aerodinámicas, coincide con el cambio en la pendiente de sustentación, es decir, con el comienzo de la pérdida del ala, con lo cual es esperable que aparezcan este tipo de inestabilidades. 
“Resultados y Análisis”

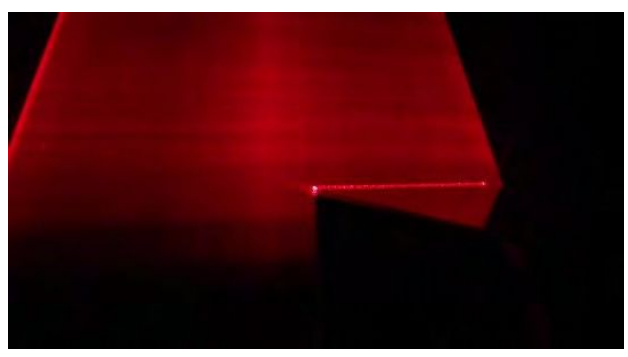

$4^{\circ}$

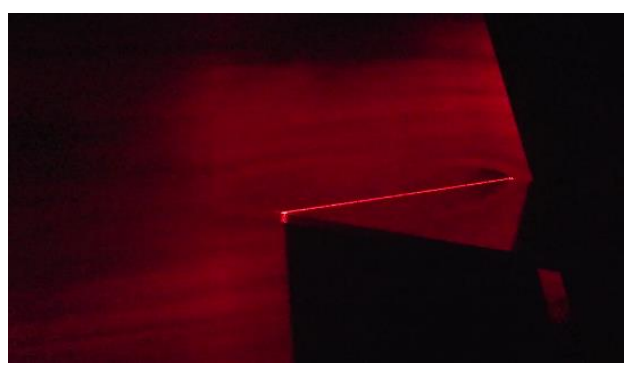

$10^{\circ}$

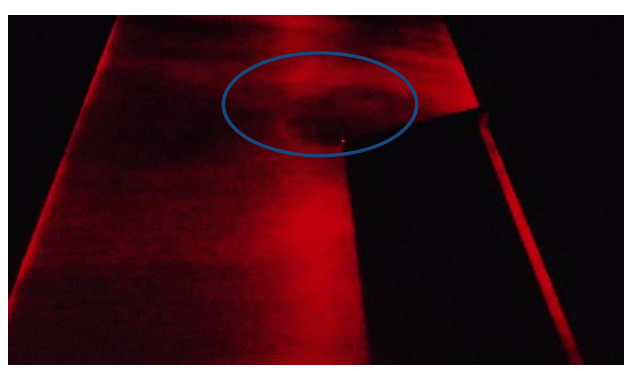

$15^{\circ}$

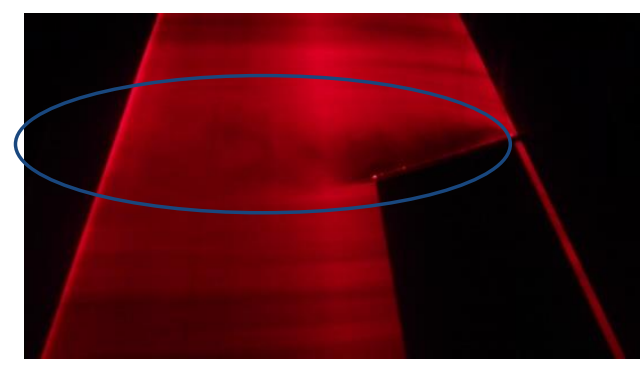

$30^{\circ}$

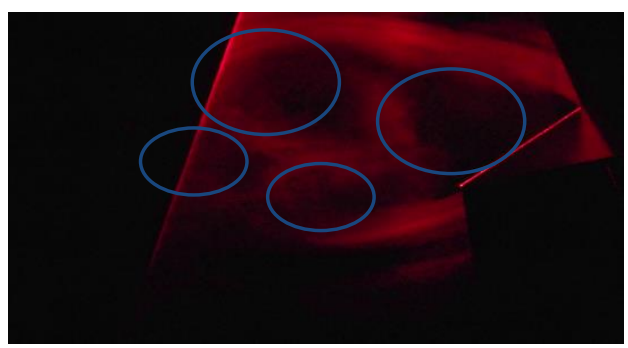

$45^{\circ}$

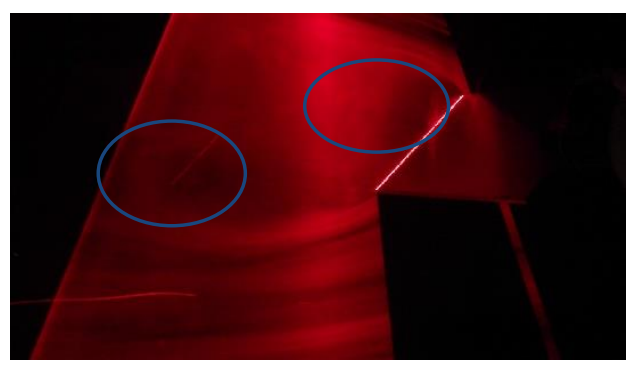

Figura 3.20. Visualización con humo para distintos ángulos de ataque en estacionario. 

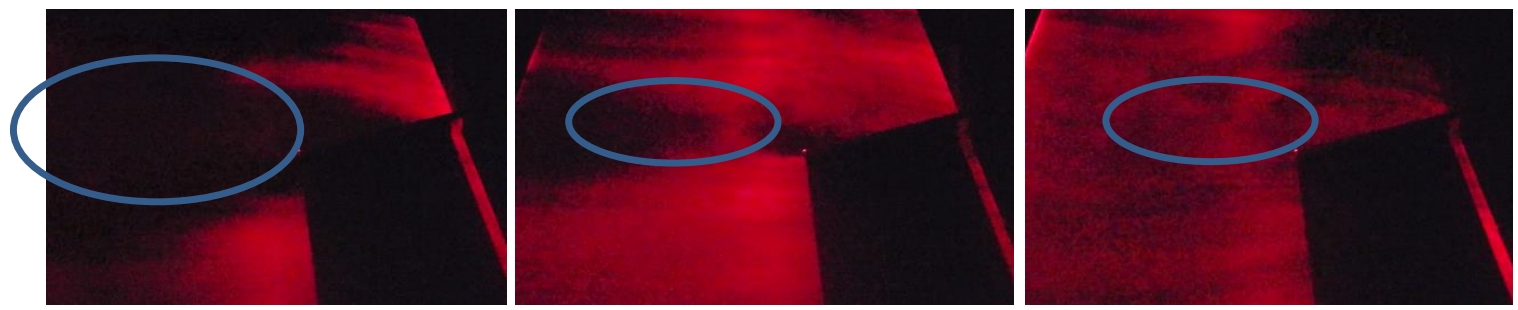

Figura 3.21. Secuencia temporal para ángulo de ataque de $10^{\circ}$.

\subsection{Condiciones dinámicas tipo Rampa}

En la siguiente secuencia de imágenes se visualiza el caso dinámico de frecuencia reducida $(K) 0,025$, caso de rotación más rápida para el Reynolds 23.000. El tiempo entre imágenes de $1 / 30$ segundos $(0,0333 \mathrm{~s})$, lo que equivale a un tiempo convectivo (tiempo adimensionalizado con la cuerda y la velocidad de la corriente libre) de 2,46 . Esto quiere decir que entre imagen e imagen, una partícula si se mueve a la velocidad de la corriente libre, recorre 2,46 cuerdas de distancia. En la secuencia de imágenes, la foto de la esquina superior izquierda corresponde al instante temporal 0 , perfil con $0^{\circ}$ de ángulo de ataque, y la secuencia va creciendo primero en sentido descendente y luego lateralmente, es decir, la foto de la segunda fila columna izquierda es la foto del segundo instante luego del instante inicial. Al ver la segunda columna (salvo la primera imagen) y la tercera columna, estamos visualizando con el ala fija en cuarenta y cinco grados de ángulo de ataque, para analizar el fin del efecto transitorio y el estacionario posterior. En la secuencia se puede observar como para un ángulo de ataque de $18^{\circ}$ el flujo comienza a formar un vórtice, que se mantiene adherido a la superficie del ala hasta los $45^{\circ}$, donde se desprende y comienza a viajar aguas abajo. Luego en el instante de tiempo adimensional 14,7, se puede observar la formación de un vórtice de borde de fuga para luego generarse una estela turbulenta, también conocida como capa de corte.

\subsubsection{Mediciones en Placa Plana}

Como fue mencionado anteriormente, el objetivo principal de este trabajo es la determinación de los esfuerzos en dos modelos de ala, uno correspondiente a una placa plana de $3 \%$ de espesor y un perfil aerodinámico de bajo número de Reynolds. En esta sección encontraremos los resultados obtenidos para la placa plana con borde de ataque y de fuga romos, sin ningún tratamiento de suavizado. Dentro de esta sección se dividirán los resultados obtenidos en forma estática, con respecto a los ensayos dinámicos, comenzando con el análisis de los resultados encontrados para condiciones estáticas. Dentro de cada una de las secciones, se mostrarán los resultados para cada condición de turbulencia y luego al final la comparación de resultados para evidenciar el efecto de esta. 

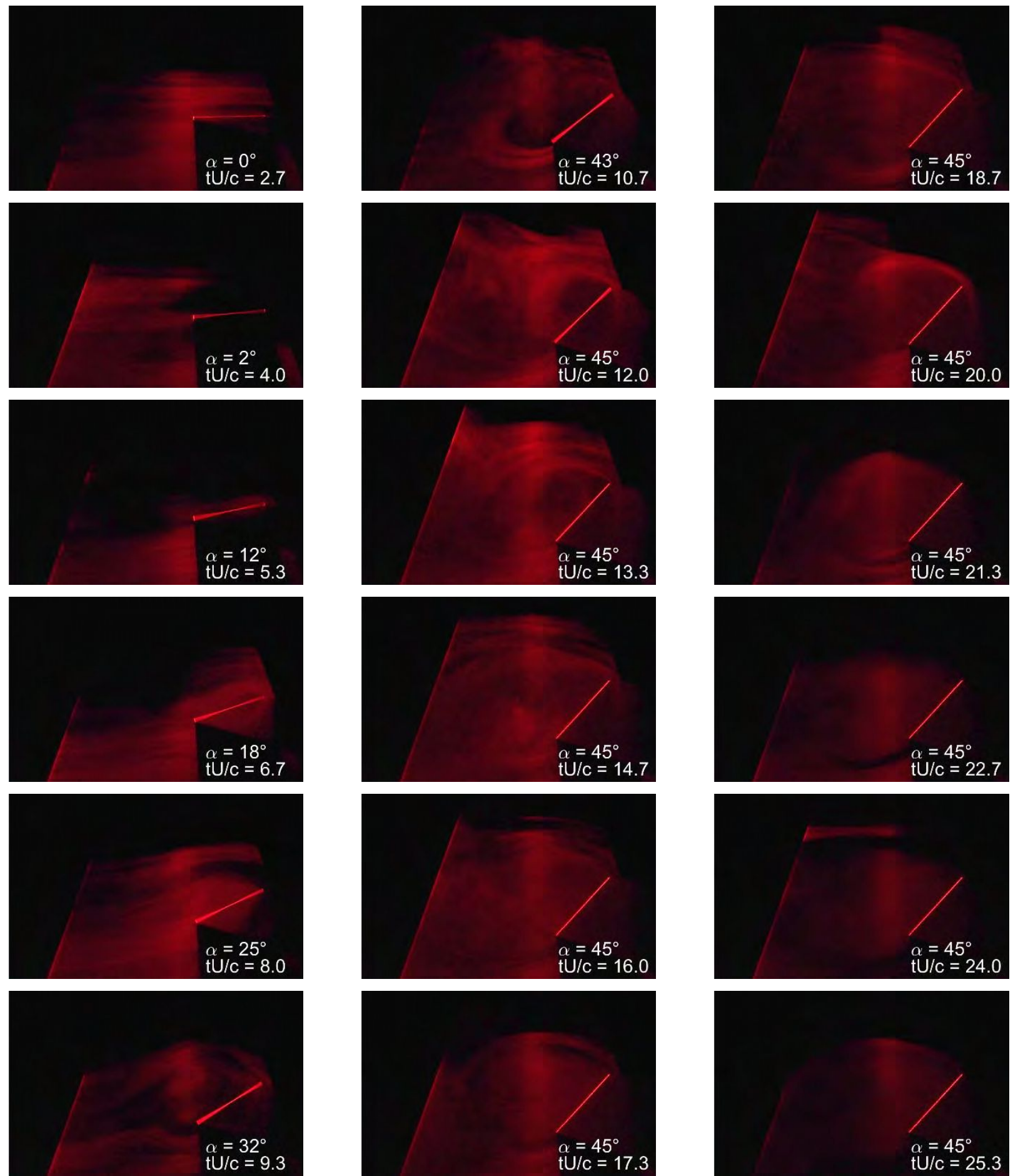

Figura 3.22. Secuencia de imágenes del caso dinámico con frecuencia reducida de $\mathbf{0 . 0 2 5}$ para un Re de 23000 . La secuencia comienza en la esquina superior izquierda y evoluciona temporalmente con un paso temporal de 0,033 segundos siguiendo una disposición vertical.

\subsubsection{Ensayos estáticos}

\subsection{Ensayos con mínima intensidad de turbulencia}

Se realizaron las mediciones a los distintos números de Reynolds especificados anteriormente (23, 46 y 57 mil), repitiendo tres veces cada medición. A continuación, se muestran los resultados medios obtenidos de 


\section{"Estudio aerodinámico experimental en flujo turbulento de bajo Reynolds sobre alas con movimiento de cabeceo"}

dichos ensayos, así como sus barras de desvío estándar. Además, se muestra la evolución de la eficiencia aerodinámica $(1 / \mathrm{d})$ en función del número de Reynolds y del ángulo de ataque. En la Figura 3.23 se puede observar los valores de coeficiente de sustentación y resistencia en función del ángulo de ataque, para los distintos números de Reynolds ensayados. Al analizar el valor del coeficiente de sustentación, se presenta una zona de incremento lineal entre el coeficiente de sustentación con el ángulo de ataque, hasta que el flujo comienza a desprenderse, para un valor menor a $10^{\circ}$, aunque sigue incrementando el mismo a costas de un gran incremento de la resistencia, como es esperado. También se observa una mayor resistencia al aumentar el número de Reynolds para altos valores de ángulo de ataque. A su vez, se observa una similitud entre el valor de resistencia y sustentación para el caso de $45^{\circ}$ de ángulo de ataque. Esto se condice con lo esperado, dado que la componente normal a la placa plana es la que genera la mayor componente de fuerza mientras que la axial es siempre una componente muy baja. Además, si comparamos con la literatura relacionada, los resultados obtenidos son muy similares a los de otros autores (Figura 3.26 y Figura 3.27 - ver "flat plate"). Si observamos la curva de eficiencia vs. ángulo de ataque (Figura 3.24), podemos encontrar bajos valores de eficiencia, comúnmente hallados en números bajos de Reynolds debido a las grandes resistencias viscosas dominantes. Si vemos la pendiente de la curva $\mathrm{Cl}$ vs $\alpha$ (Figura 3.25), podemos observar que la misma tiene un valor de $1.4 \pi$ $\sim 1.5 \pi$, hasta el valor de $6^{\circ}$ de ángulo de ataque, donde dicho valor cae bruscamente por presencia de desprendimientos de capa límite desde el borde de ataque, como se puede ver mediante las visualizaciones realizadas con humo y condice con el comportamiento del coeficiente de sustentación. Este valor de pendiente es menor al valor teórico de la pendiente de una placa plana delgada $(2 \pi)$, pero se condice dado que el valor teórico es para flujo potencial, donde se desprecian los efectos viscosos, y las condiciones de ensayo en este trabajo, producto de los bajos números de Reynolds, no condicen con dicha hipótesis, lo que produce una disminución en la pendiente de sustentación. A su vez, podemos destacar el valor del coeficiente de sustentación y resistencia para un ángulo de $45^{\circ}$, el cual nos servirá para comparar con los resultados dinámicos, dichos valores se pueden ver en la Tabla 3.2.

Tabla 3.2 Coeficientes aerodinámicos para ángulo de ataque $45^{\circ}$ con mínima turbulencia.

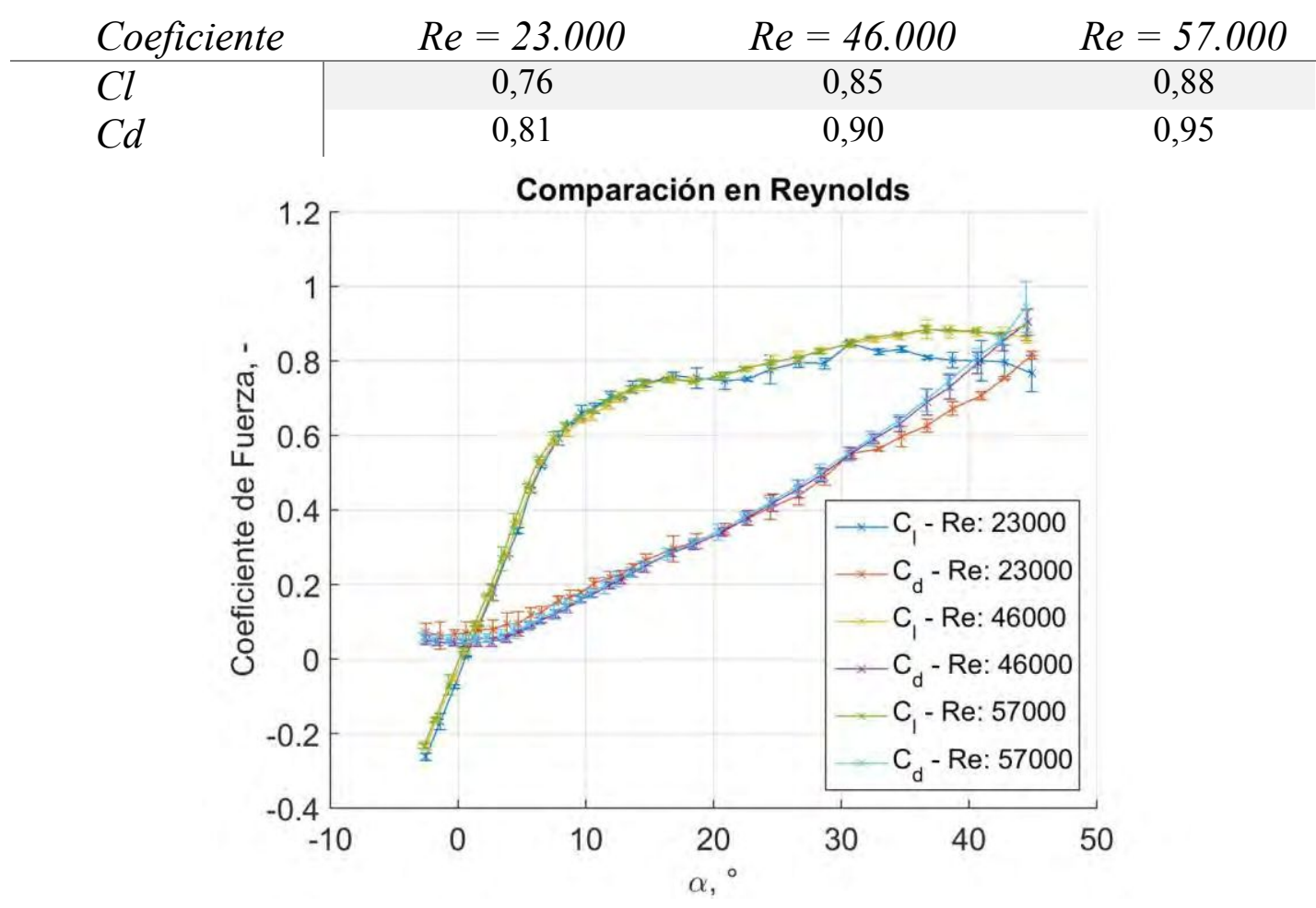

Figura 3.23. Curva CL y Cd versus ángulo de ataque para distintos Reynolds con mínima intensidad de turbulencia. 


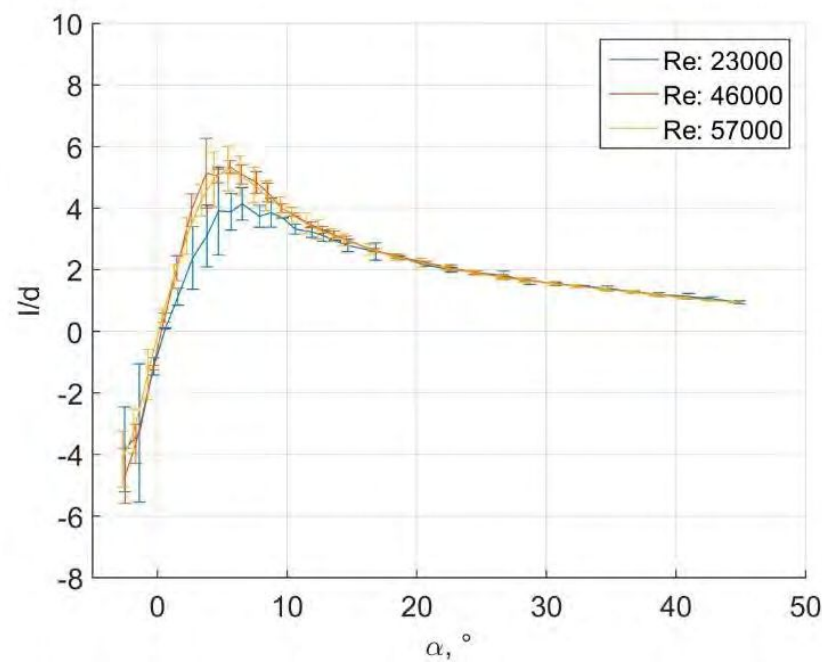

Figura 3.24. Eficiencia aerodinámica en función del ángulo de ataque y del número de Reynolds. Mínima intensidad de turbulencia

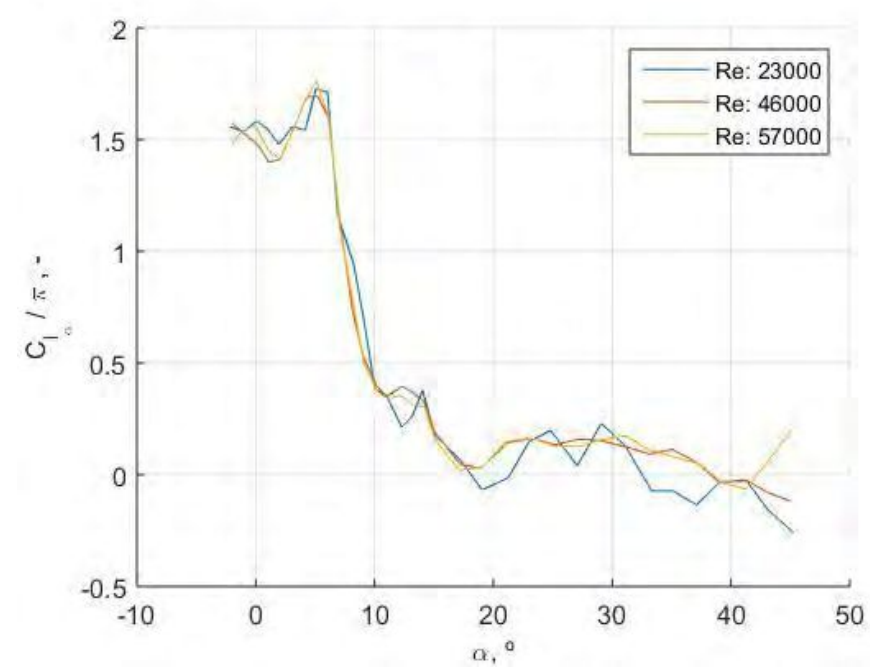

Figura 3.25. Pendiente de la curva de sustentación en función del ángulo de ataque. Mínima intensidad de turbulencia
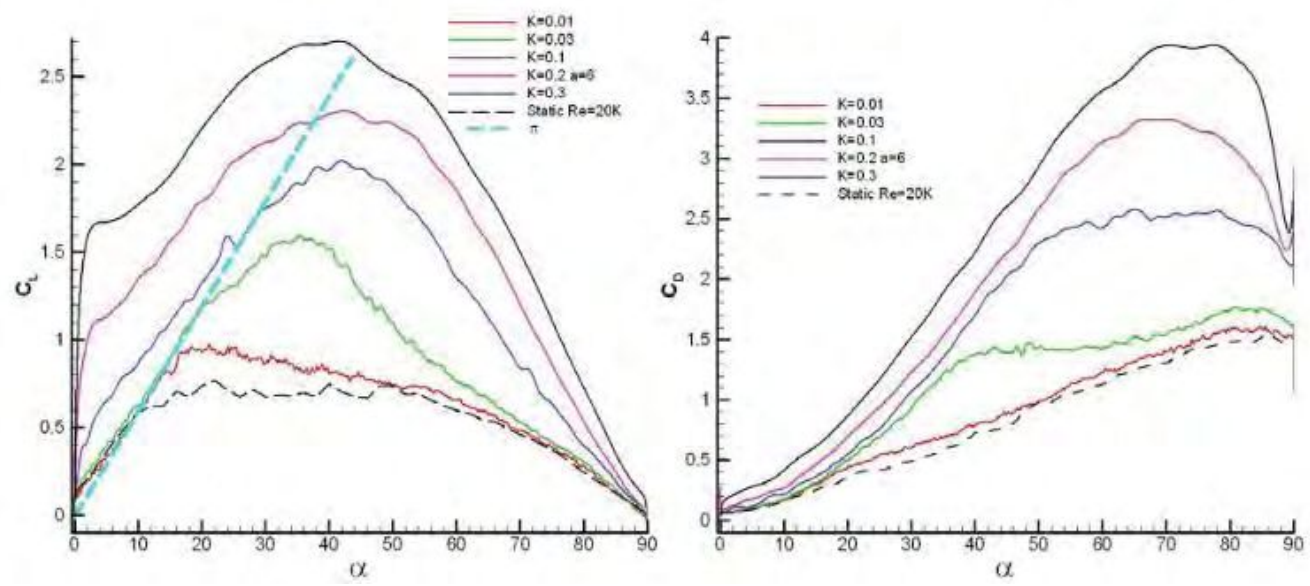

Figura 3.26. Coeficiente de sustentación y Resistencia para distintos movimientos y ángulos de ataque. Grandlund et al. 2011. Re: 20000. Flujo Laminar. 


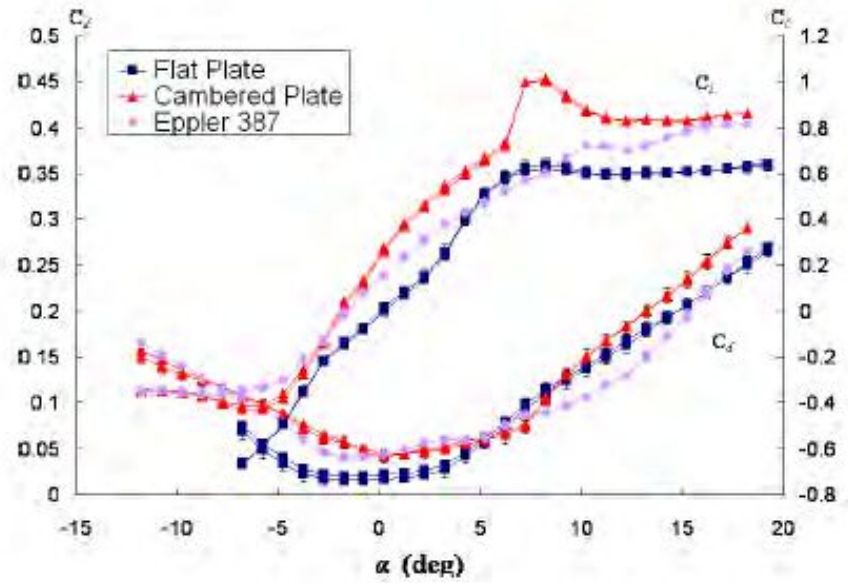

(a) With Endplates - 2D airfoil

Figura 3.27. Cl y CD para distintos perfiles. Re: 10000. McArthur, 2016. Flujo Laminar.

\subsection{Ensayos con máxima intensidad de turbulencia $(I=1 \%)$}

De igual manera que con los ensayos estáticos de mínima turbulencia, se repitieron los ensayos utilizando la grilla generadora de turbulencia que modifica el flujo incidente, aumentando la intensidad de turbulencia hasta aproximadamente el valor del $1 \% \mathrm{y}$ acotando las escalas turbulentas al valor de aproximadamente $15 \mathrm{~mm}$.

De esa manera, se obtuvieron las gráficas de los coeficientes aerodinámicos en condiciones estáticas y se compararon los resultados con los resultados obtenidos para una turbulencia menor.

Al ver los resultados con la nueva configuración turbulenta, se pueden encontrar diferencias respecto a la condición de flujo "laminar". Dado que se pueden observar diferencias al comparar entre los distintos números de Reynolds, que antes, estas diferencias no eran importantes. A su vez, se observa un comportamiento muy diferenciado entre el número de Reynolds de 23000 y los números de Reynolds más altos, donde se observa un coeficiente de resistencia mucho mayor, que para la condición de baja intensidad de turbulencia no se apreciaba. Un resumen con estos resultados se encuentra en la Tabla 3.3.

Tabla 3.3. Coeficientes aerodinámicos para ángulo de ataque $45^{\circ}$ con intensidad de turbulencia de $1 \%$.

\begin{tabular}{|c|c|c|c|}
\hline Coeficiente & $\begin{array}{c}R e= \\
23.000\end{array}$ & $\begin{array}{c}R e= \\
46.000\end{array}$ & $\begin{array}{c}R e= \\
57.000\end{array}$ \\
\hline $\mathrm{Cl}$ & 0,72 & 0.85 & 0.91 \\
\hline Diferencia porcentual respecto "laminar" & $-5 \%$ & $0 \%$ & $3 \%$ \\
\hline$C d$ & 1,00 & 1,03 & 1.03 \\
\hline Diferencia porcentual respecto "laminar" & $23 \%$ & $14 \%$ & $9 \%$ \\
\hline
\end{tabular}




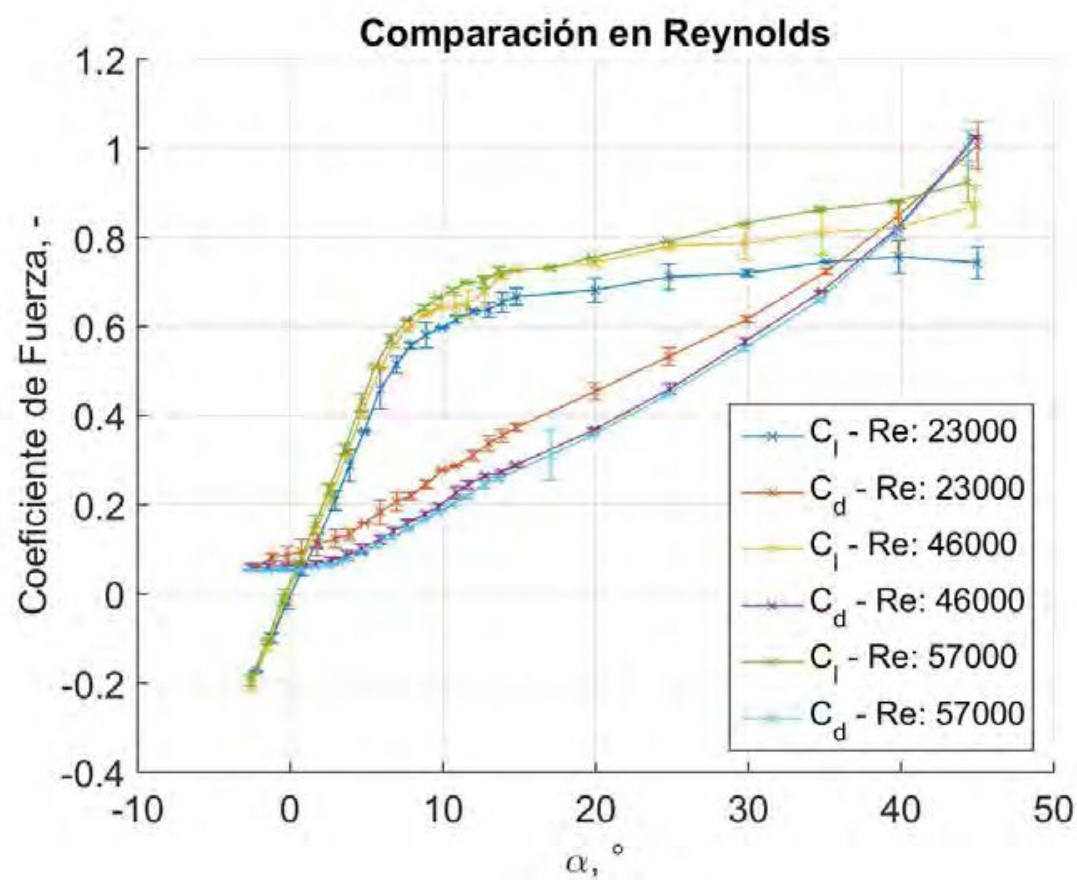

Figura 3.28. Coeficientes aerodinámicos para distintos números de Reynolds con alta turbulencia.

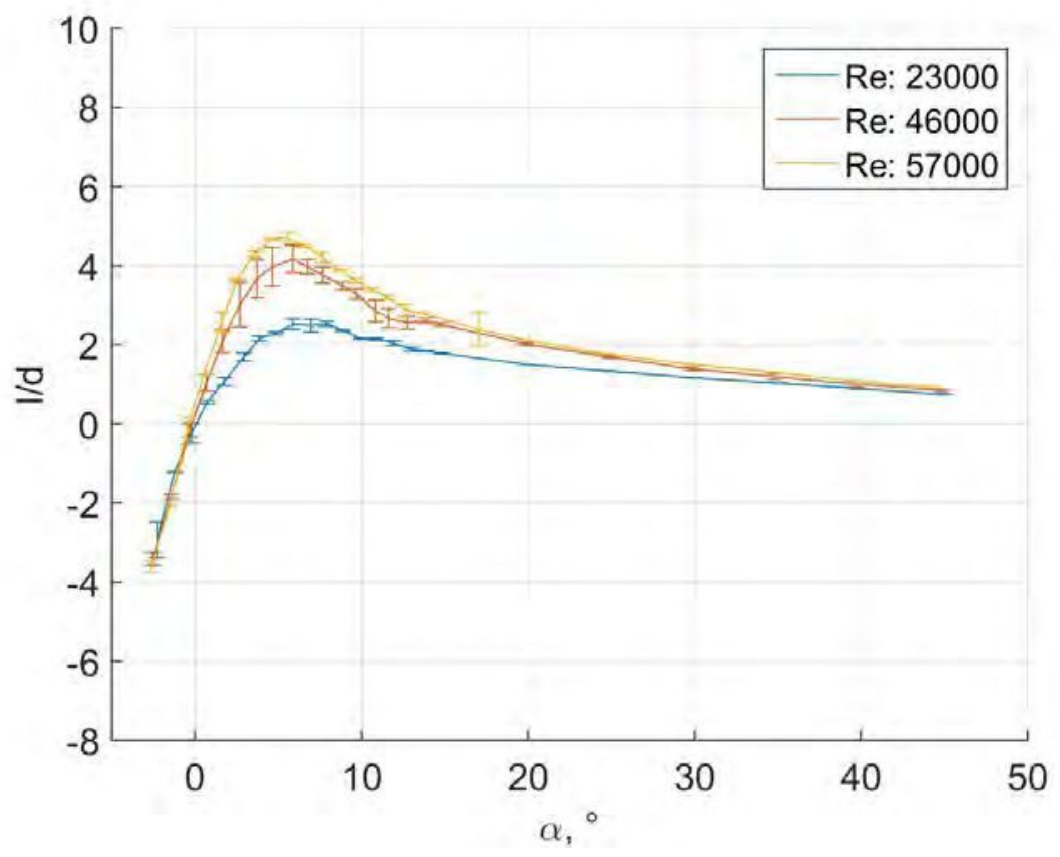

Figura 3.29. Eficiencia aerodinámica para los distintos números de Reynolds con alta turbulencia. 


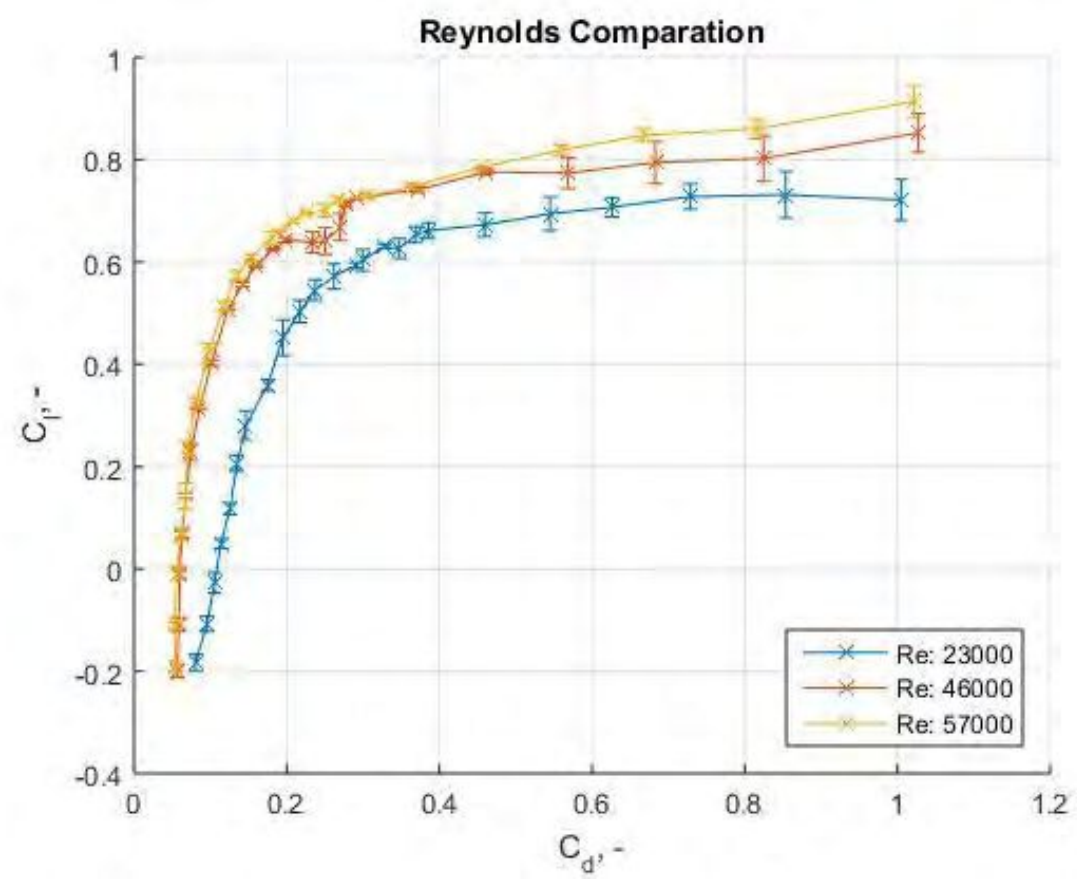

Figura 3.30. Polar de coeficientes aerodinámicos para distintos Reynolds con alta turbulencia.

\subsection{Comparación Estática entre diferentes turbulencias}

A su vez, se realizaron comparaciones de estos resultados con los obtenidos anteriormente para poder analizar la influencia de la turbulencia en las cargas aerodinámicas.

De los resultados obtenidos (Figura 3.31), se puede observar como a medida que se aumenta el número de Reynolds, los efectos de la turbulencia empiezan a mitigarse. Para el número de Re más bajo ensayado, es apreciable el efecto de la turbulencia para todos los ángulos de ataque en el coeficiente de resistencia, pero sobre el coeficiente de sustentación, sólo se encuentran diferencias cuando la placa plana empieza a mostrar desprendimientos (ángulo de ataque mayor a $8^{\circ}$ ), siendo menor el coeficiente de sustentación cuando se aumenta la intensidad de la turbulencia. Este resultado contradice los resultados expuestos por otros autores mencionados en los antecedentes de este trabajo, donde sus resultados demostraban que, al aumentar la intensidad de turbulencia, el coeficiente de sustentación máximo se incrementa. La razón principal que explica haber encontrado un resultado opuesto es que la placa plana al tener un borde de ataque romo, entra en pérdida de forma muy brusca, desprendiéndose por completo la capa límite, mientras que en perfiles aerodinámicos, se puede energizar la capa límite en la zona cercana a la entrada en pérdida, aumentando el ángulo de ataque de entrada en pérdida. Para los números de Reynolds de 46000 y 57000 no se aprecian efectos significativos en los resultados encontrados. Hay un ligero incremento del coeficiente de resistencia en ambos casos, pero no es una diferencia muy abrupta. 
Re: 23000
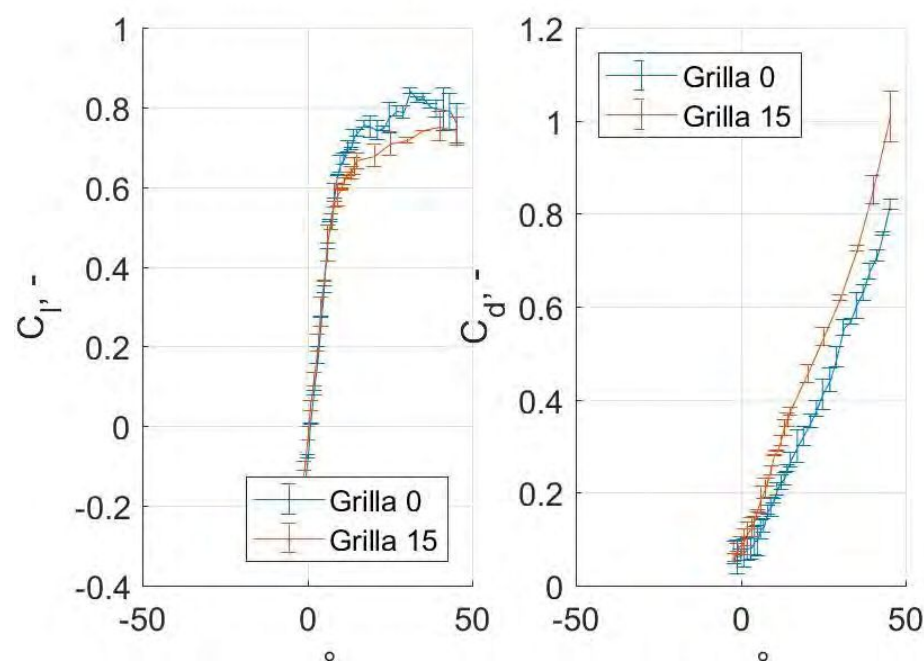

Re: 46000
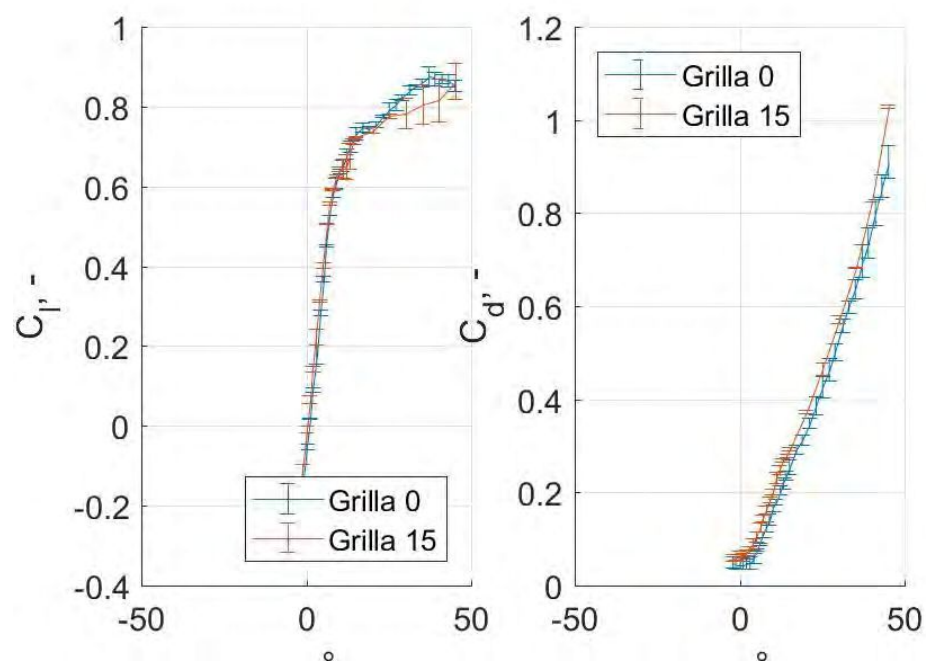

Re: 57000
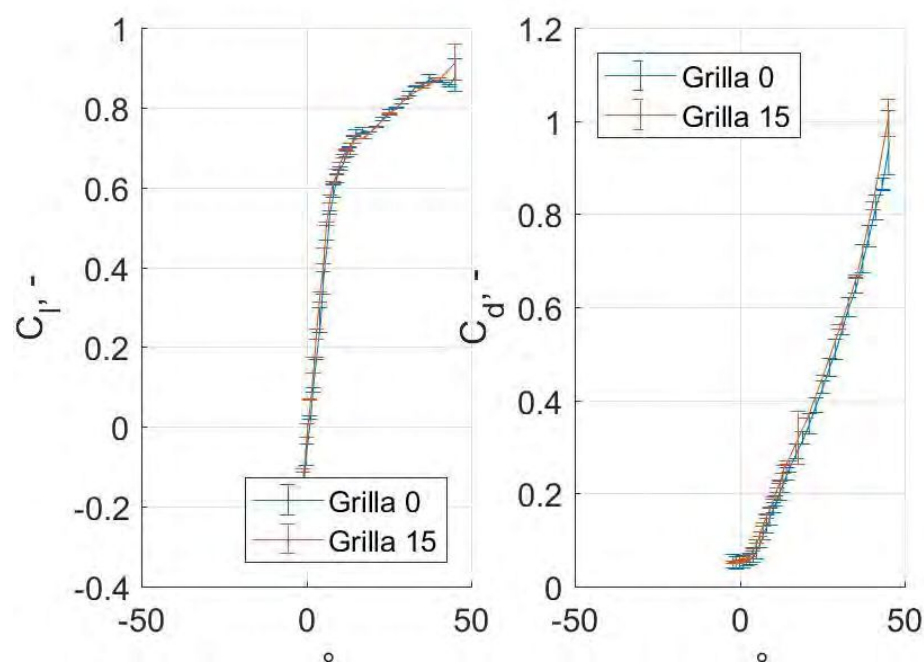

Figura 3.31.Comparación de coeficientes aerodinámicos para distintas turbulencias. Las barras indican $1 \mathrm{STD}$ 


\section{"Estudio aerodinámico experimental en flujo turbulento de bajo Reynolds sobre alas con movimiento de cabeceo"}

\subsubsection{Ensayos dinámicos: Movimiento continuo}

\subsection{Ensayos con mínima intensidad de turbulencia}

En esta sección se muestran los resultados del procesamiento de las mediciones de cargas realizadas sobre la placa plana con movimiento de cabeceo continuo. En las siguientes figuras se muestran los resultados medios de los distintos casos analizados, en función del tiempo, adimensionalizado con el periodo del movimiento (Figura 3.32), y en función del ángulo de ataque (Figura 3.33), comparando dichos resultados con los encontrados para la condición de ángulo de ataque estático.

Para los gráficos en función del tiempo adimensional, Figura 3.32, hay que tener presente que a partir de 0,1 el ángulo incrementa, hasta 0,4 , donde comienza la zona de ángulo de ataque constante en su valor máximo $\left(15^{\circ}\right)$; en 0,6 el movimiento ya se encuentra en su régimen de velocidad de cambio de ángulo de ataque constante, decreciendo, hasta 0,9 donde comienza el proceso de desaceleración para llegar a los $0^{\circ}$ de ángulo de ataque hasta el valor de 1 en el tiempo adimensional.

Para el caso de las cargas en función del ángulo de ataque, Figura 3.33, simplemente se obtiene la media de los valores de carga para cada ángulo de ataque en el ciclo ascendente y descendente, con lo cual se puede observar la aparición del efecto de histéresis en la curva, el cual se intensifica a medida que se aumenta la frecuencia reducida. Además, se puede observar el retraso en la entrada en pérdida en la placa, producto de los efectos dinámicos.
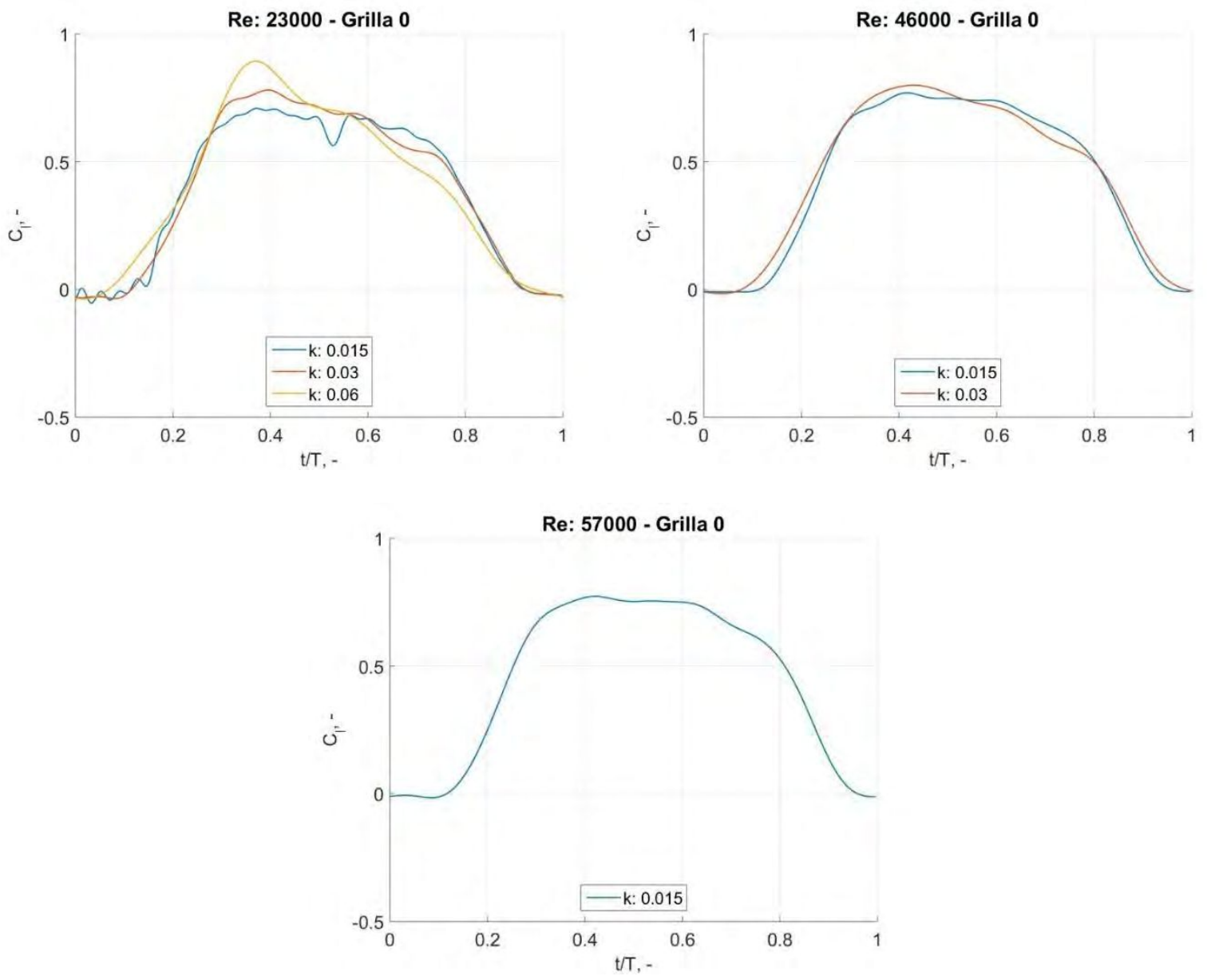

Figura 3.32. Evolución de la carga aerodinámica en el tiempo adimensional 

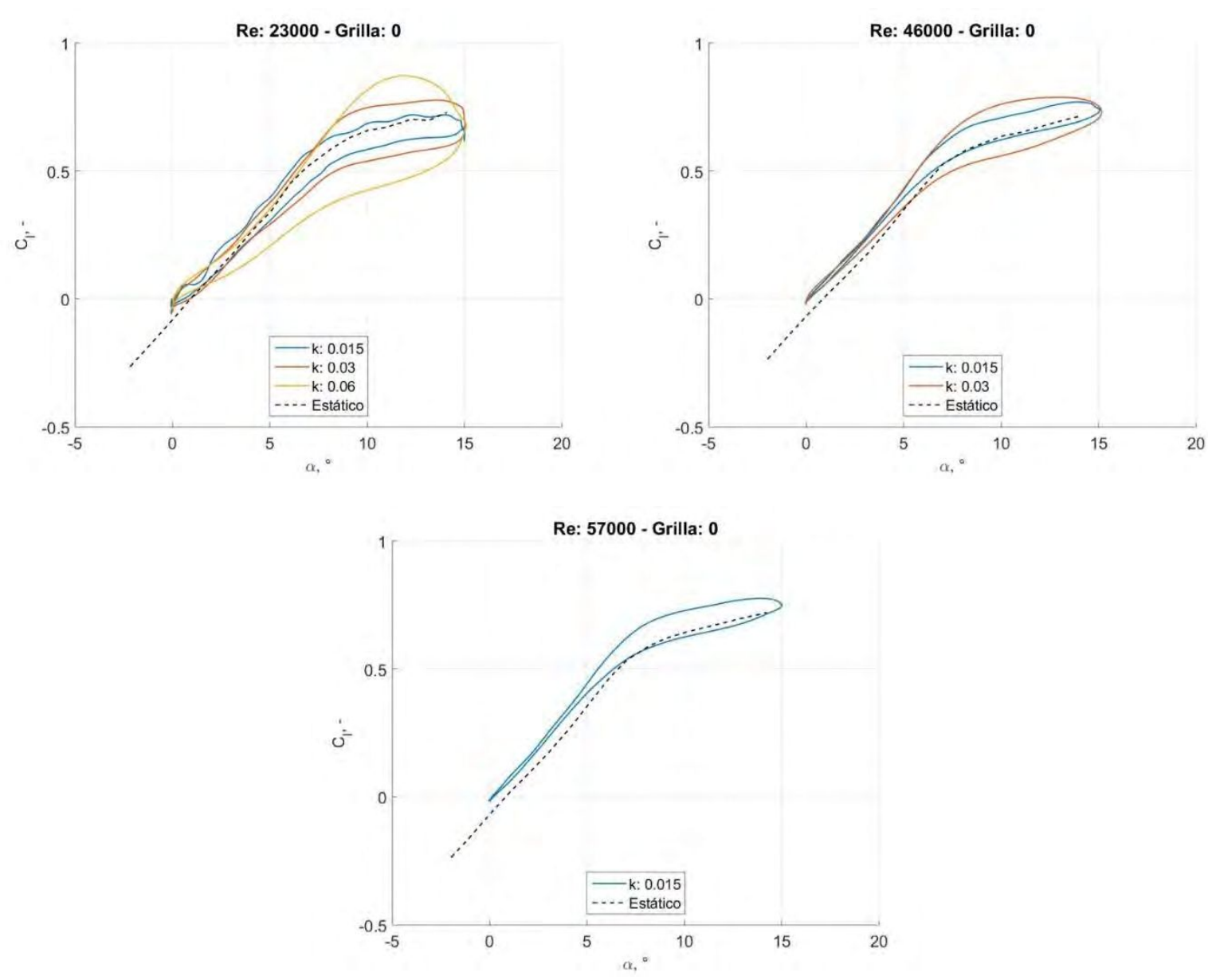

Figura 3.33. Coeficiente de sustentación en función del ángulo de ataque para distintas frecuencias reducidas y número de Reynolds.

\subsection{Ensayos con máxima intensidad de turbulencia (I=1\%)}

Se repitieron los ensayos para la configuración de máxima intensidad de turbulencia, utilizando el mismo método de tarado dinámico y el mismo filtro digital para eliminar las componentes fluctuantes producto de las vibraciones mecánicas. En las siguientes figuras se muestran de forma análoga al caso de mínima intensidad de turbulencia los resultados en función del tiempo adimensional y del ángulo de ataque. Luego, se compararán los resultados entre las distintas intensidades turbulentas para evidenciar el efecto o no de la turbulencia en el fenómeno estudiado.

En la Figura 3.34 se puede observar como al incrementar la frecuencia reducida, nuevamente se produce un incremento en el coeficiente de sustentación máximo, pero en la carrera descendente $(t / T>0,5)$ el fenómeno es el contrario, resultando un valor de sustentación menor con el aumento de frecuencia reducida. Si observamos el comportamiento de la carga aerodinámica en función del ángulo de ataque, ver Figura 3.35, se puede apreciar el retraso de la entrada en pérdida (comportamiento lineal del $\mathrm{Cl}$ con $\alpha$ ) con el incremento de la frecuencia reducida. A su vez, se puede apreciar el incremento en la histéresis en la carga, al igual que con la mínima intensidad de turbulencia. 

de cabeceo"
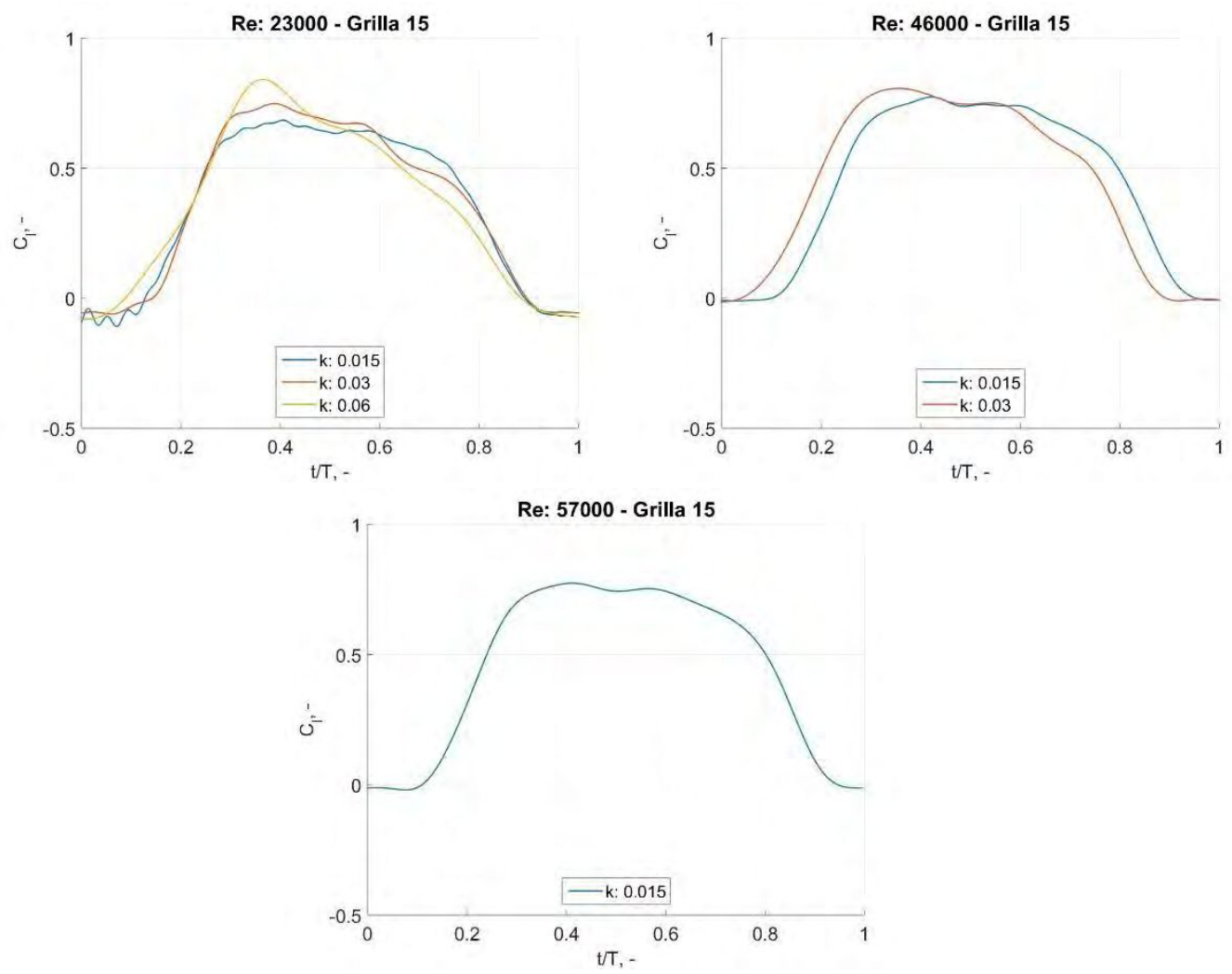

Figura 3.34. Evolución de las cargas aerodinámicas en función del tiempo adimensional.
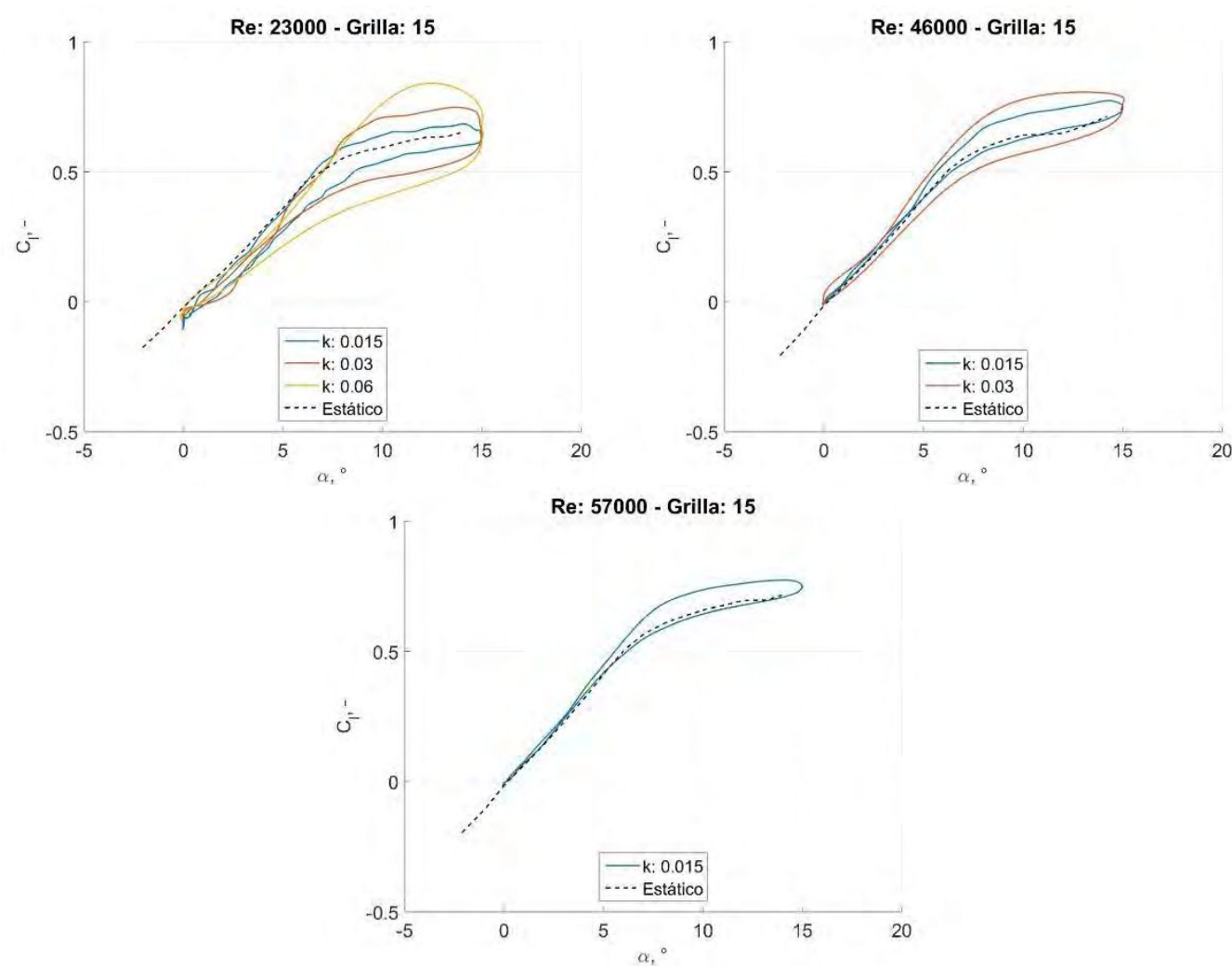

Figura 3.35. Cargas aerodinámicas en función del ángulo de ataque. 


\subsection{Comparación del efecto de la turbulencia}

Cómo es de gran interés para este trabajo obtener de forma explícita la influencia de la turbulencia sobre el fenómeno de movimiento continuo, a continuación, se pone en manifiesto en las siguientes figuras la influencia de la turbulencia incidente en las cargas aerodinámicas, en función del ángulo de ataque.

Al poder comparar de forma directa la evolución de las cargas para ambas condiciones, no se observan cambios en la sustentación desarrollada por el ala para los distintos niveles de turbulencia, producto de la no estacionalidad. Es decir, además del cambio en las cargas aerodinámicas que fueron halladas en la condición estática, no se observan otras modificaciones producto de los efectos dinámicos, dado que se observa como en la zona de ángulo de ataque mayor al de entrada en pérdida estática la tendencia sigue a las cargas estacionarias, aunque con la histéresis esperada producto del fenómeno dinámico. En los gráficos, la leyenda grilla 0 indica el caso con mínima intensidad de turbulencia, mientras que el caso Grilla 15 indica la condición de flujo con máxima intensidad de turbulencia.
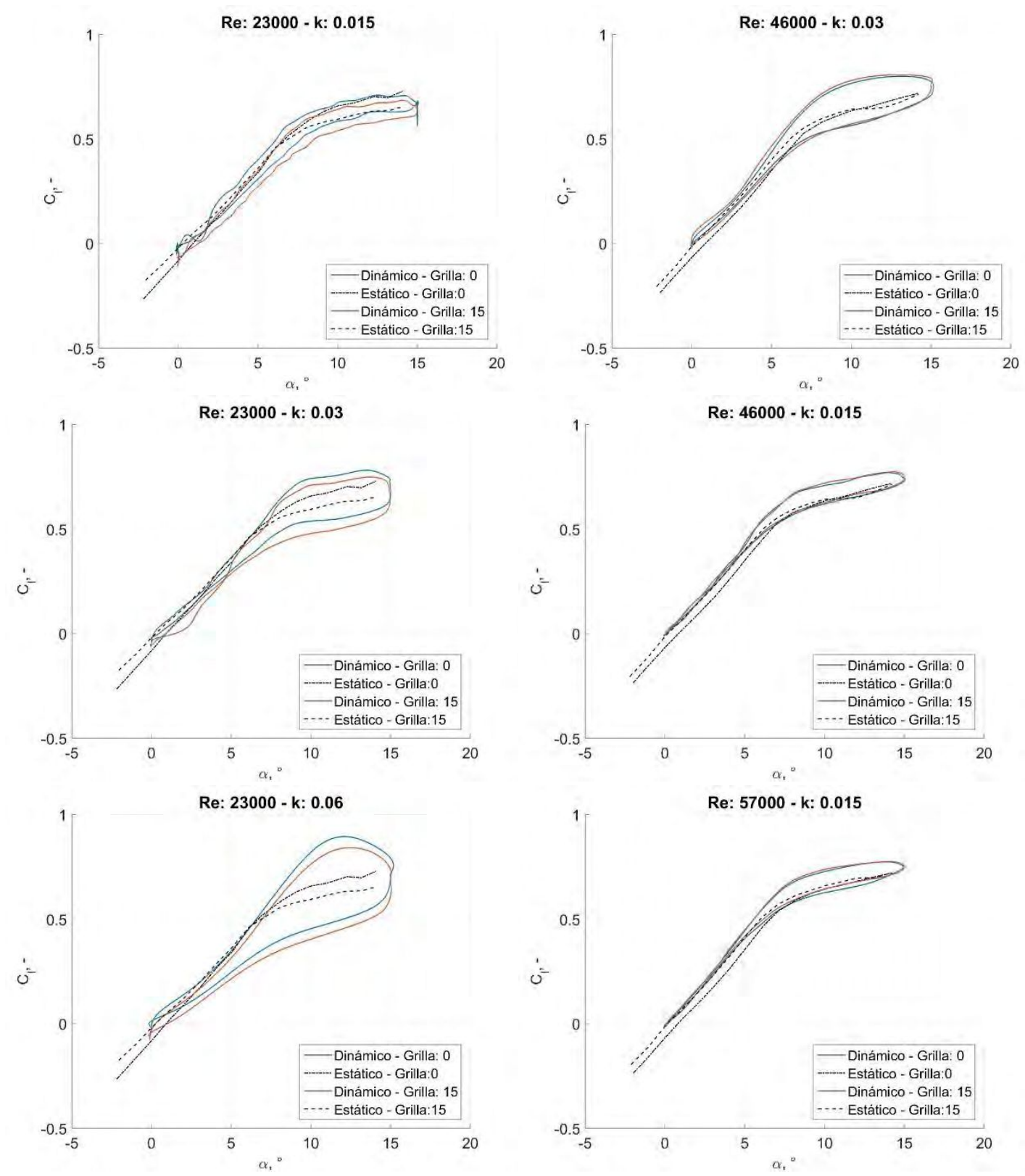

Figura 3.36. Comparación de las cargas aerodinámicas para distintos niveles de intensidad de turbulencia. 
Tabla 3.4. Valores de sustentación para Reynolds 23.000, estático y dinámico para placa plana.

\begin{tabular}{l|c|cc|cc|cc|}
\multicolumn{3}{c}{ Estático } & \multicolumn{2}{c}{$k=0,015$} & \multicolumn{2}{c}{$k=0,03$} & \multicolumn{2}{c}{$k=06$} \\
\hline & $\mathrm{C}_{1}$ máximo & $\mathrm{C}_{1}$ máximo & $\Delta \mathrm{C}_{1}$ & $\mathrm{C}_{1}$ máximo & $\Delta \mathrm{C}_{1}$ & $\mathrm{C}_{1}$ máximo & $\Delta \mathrm{C}_{1}$ \\
Grilla 0 & 0,73 & 0,72 & 0,1 & 0,77 & 0,18 & 0,87 & 0,4 \\
Grilla 15 & 0,65 & 0,69 & 0,1 & 0,74 & 0,17 & 0,85 & 0,35 \\
\cline { 2 - 8 }
\end{tabular}

Tabla 3.5. Cociente entre histéresis $\left(\Delta \mathrm{C}_{\mathrm{l}}\right)$ y el coeficiente máximo de sustentación.

\begin{tabular}{l|c|c|c}
\multicolumn{1}{c}{$k=0,015$} & $k=0,03$ & $k=0,06$ \\
\hline Grilla 0 & 0,139 & 0,234 & 0,460 \\
Grilla 15 & 0,145 & 0,230 & 0,412 \\
\hline
\end{tabular}

En la Tabla 3.4 se pueden observar los valores más importantes para poder comparar el efecto de la turbulencia, tanto para condiciones estáticas como dinámicas. En dicha tabla se presentan los resultados para la condición donde se pueden observar mayores diferencias en la evolución de las cargas, en función de los resultados de la Figura 3.36, es decir, para el número de Reynolds 23.000. El efecto de la turbulencia genera poca influencia en la histéresis de las cargas dinámicas, aunque para la condición de máxima frecuencia reducida, la histéresis se redujo un $12 \%$, mientras que para los demás casos es despreciable su efecto. Sin embargo, si se considera el cociente entre la histéresis $\left(\Delta \mathrm{C}_{1}\right)$ con respecto al máximo coeficiente de sustentación, ver Tabla 3.5, esta relación se mantiene aproximadamente constante para todos los casos evaluados. A su vez, queda en evidencia el efecto que produce la turbulencia en el coeficiente de sustentación máximo, reduciendo en todos los casos el máximo coeficiente de sustentación, al igual que para la condición estática, aunque a medida que se incrementa la frecuencia reducida, este efecto se minimiza.

\subsubsection{Mediciones en Perfil SD8020}

En esta sección se presentan los resultados obtenidos en las mediciones de carga aerodinámica para el modelo alar con el perfil Selig Donovan 8020, en las mismas condiciones de ensayo que la placa plana. En primera instancia se presentan los resultados para la condición de ángulo de ataque estático, para luego ahondar en los resultados para los ensayos dinámicos. Nuevamente, para la condición dinámica se utiliza el mismo método de tarado de la balanza, así como los filtros aplicados a las señales para mitigar los efectos de vibración del ala.

\subsubsection{Ensayos estáticos}

Se realizaron las mediciones a los distintos números de Reynolds especificados anteriormente, repitiendo 3 veces cada medición. A continuación, se muestran los resultados medios obtenidos de dichos ensayos, así como sus barras de desvío estándar. En la Figura 3.41 se puede observar la comparación de los resultados obtenidos con los resultados de la simulación realizada mediante el software XFoil, considerando efectos viscosos. A su vez, se representan los resultados para los distintos números de Reynolds e intensidades de turbulencia analizadas.

Como se puede observar, para todos los casos el coeficiente de sustentación obtenido en el ensayo experimental es menor al obtenido mediante la simulación numérica, lo mismo sucede con la pendiente de sustentación. Otro punto a denotar es que, para todos los números de Reynolds analizados, tanto para el ensayo como la simulación, en la región cercana al ángulo de sustentación nulo, el coeficiente de sustentación presenta un comportamiento tipo "s", que según lo que muestra la distribución de presiones en la cuerda obtenidos mediante XFoil, se debe a una zona de recirculación localizada en la zona media del extradós del perfil, ver Figura 3.37 y Figura 3.38. En las mediciones realizadas, este efecto se puede observar claramente en la pendiente de la curva de sustentación, la cual tiene un decaimiento en la zona de sustentación nula. Por otro lado, 
comparando los resultados para los dos niveles de turbulencia analizados, se puede observar que, al incrementar la intensidad, el tamaño de la burbuja se disminuye considerablemente, así como también se localiza en una zona más próxima al borde de ataque. Si bien en el nivel mayor de turbulencia se puede ver una zona de recirculación, aunque de menor tamaño lo cual trae aparejado un desvío menor respecto a la linealidad de la curva $\mathrm{Cl}$ vs $\alpha$, como se puede observar en la Figura 3.41. Para las mediciones experimentales, ese desvío se ve principalmente para el Reynolds 23000, pero no es tan importante en los Reynolds mayores. Esto se puede evidenciar al observar la Figura 3.39, donde se representa el valor de la pendiente de la curva $\mathrm{Cl}$ adimensionalizado por el valor pi versus el ángulo de ataque. Si se realiza una comparación del coeficiente de sustentación en función de la intensidad de turbulencia, según el software XFoil, los cambios más importantes son en la zona del ángulo de sustentación nulo, producto de la modificación de la burbuja de recirculación, pero no así en los valores máximos del coeficiente de sustentación. Sin embargo, en las mediciones realizadas en túnel de viento, se puede observar un incremento del coeficiente de sustentación máximo, sin importantes cambios en la zona de bajos ángulos de ataque. Dichos resultados se pueden hallar en la Figura 3.42. Estos resultados condicen con resultados de otros autores, expuestos en los antecedentes, al analizar los efectos de la turbulencia en perfiles aerodinámicos.

Cabe destacar que para las simulaciones realizadas con el software Xfoil, para modificar el nivel de turbulencia de la corriente libre se modificó el valor del $\mathrm{N}_{\mathrm{cr}}$, quién está relacionado con el factor de amplificación (N) que determina en que posición ocurre la transición laminar-turbulenta a lo largo del perfil. Este método desarrollado por van Ingen 1956, tiene ciertas limitaciones (van Ingen 2008, Drela 1989, Drela y Giles 1987) e hipótesis las cuales pueden traer desvíos con lo que ocurre físicamente. Principalmente, que se produzca la transición laminar-turbulenta, produciendo una recirculación sobre el perfil, dependerá de distintos factores, no solo de la intensidad de turbulencia, más allá de la validez del método de linealizar las perturbaciones dentro de la capa límite. Esto produce que puede ser errática la predicción de la localización y tamaño de la burbuja de recirculación, para los niveles de turbulencia en los que se realizaron los ensayos experimentales de este trabajo, hasta puede ser el caso de que dicha burbuja no se produzca. Con lo cual, los resultados expuestos con el software Xfoil, sólo se consideran como una guía general de lo que ocurre con el flujo y distribución de presiones, para poder validar los resultados encontrar experimentalmente. Evidentemente la burbuja de recirculación está presente para el valor de mínima turbulencia (verificado por la no linealidad de la curva $\mathrm{Cl}$ vs $\alpha$ ), pero no podemos asegurar que el tamaño y localización sea el calculado numéricamente. Por otro lado, es conocido y verificado con el programa, que la burbuja se achicará hasta puede que no esté presente, producto de la turbulencia de la corriente libre, dado que por las elevadas fluctuaciones se puede producir lo que se conoce como "by-pass" de la transición laminar turbulenta. Este efecto puede ser el que está ocurriendo a mayores números de Reynolds, donde esa no-linealidad desaparece, lo cual indicaría que la burbuja a desaparecido y toda la capa límite tiene características turbulentas. 
"Estudio aerodinámico experimental en flujo turbulento de bajo Reynolds sobre alas con movimiento de cabeceo"

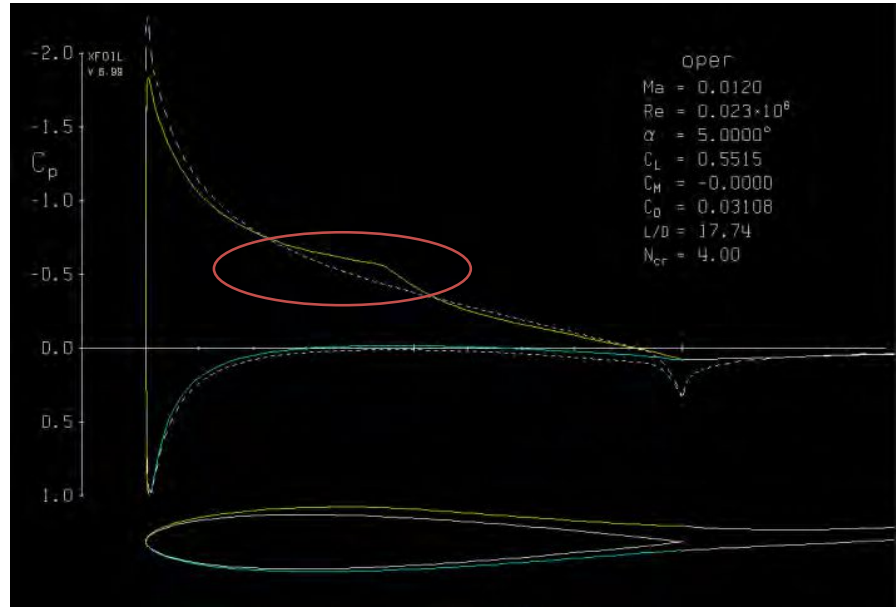

Figura 3.37. Distribución de presiones en perfil SD8020 para Reynolds $23000, \alpha=5^{\circ} \mathrm{e} \mathrm{I}=0.5 \%$.

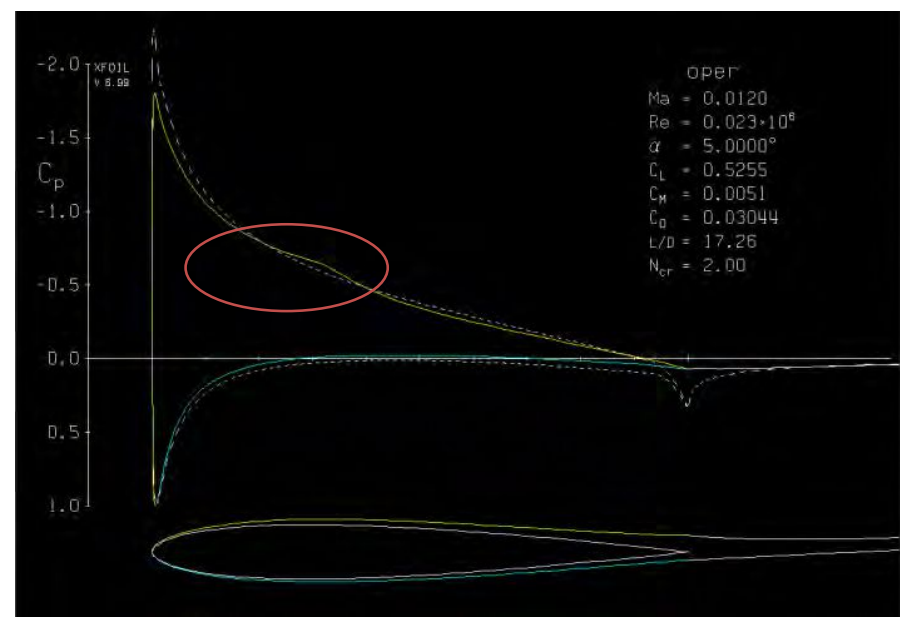

Figura 3.38. Distribución de presiones en perfil SD8020 para Reynolds $23000, \alpha=5^{\circ}$ e $I=1 \%$.
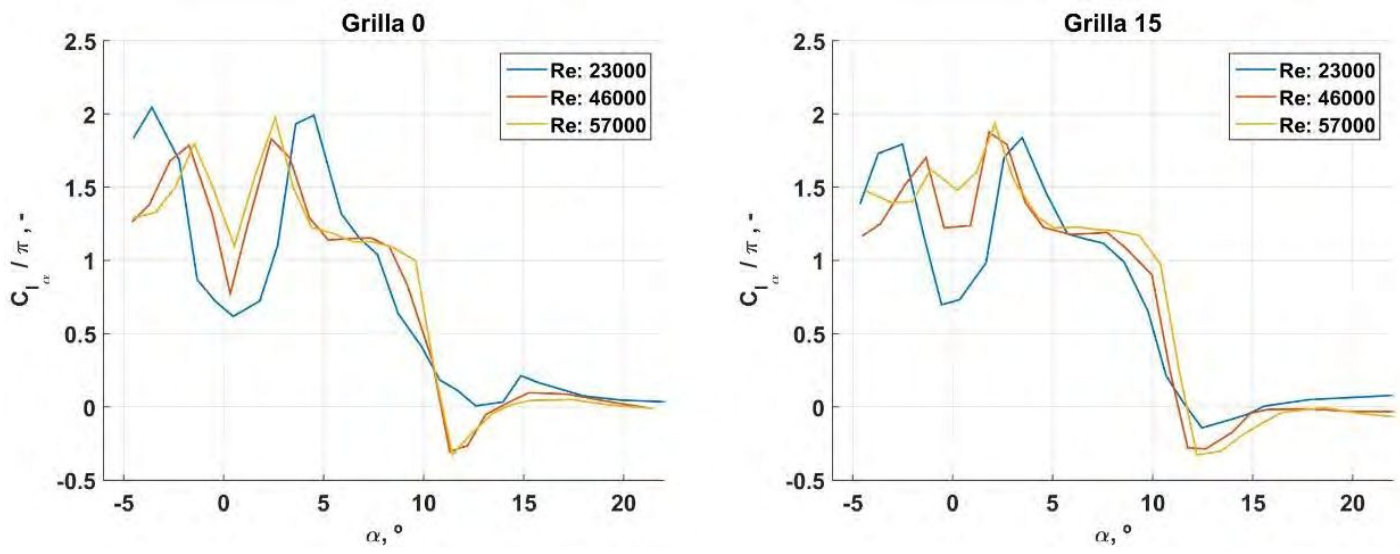

Figura 3.39. Pendiente de la curva sustentación $\alpha$ para cada ángulo de ataque. 

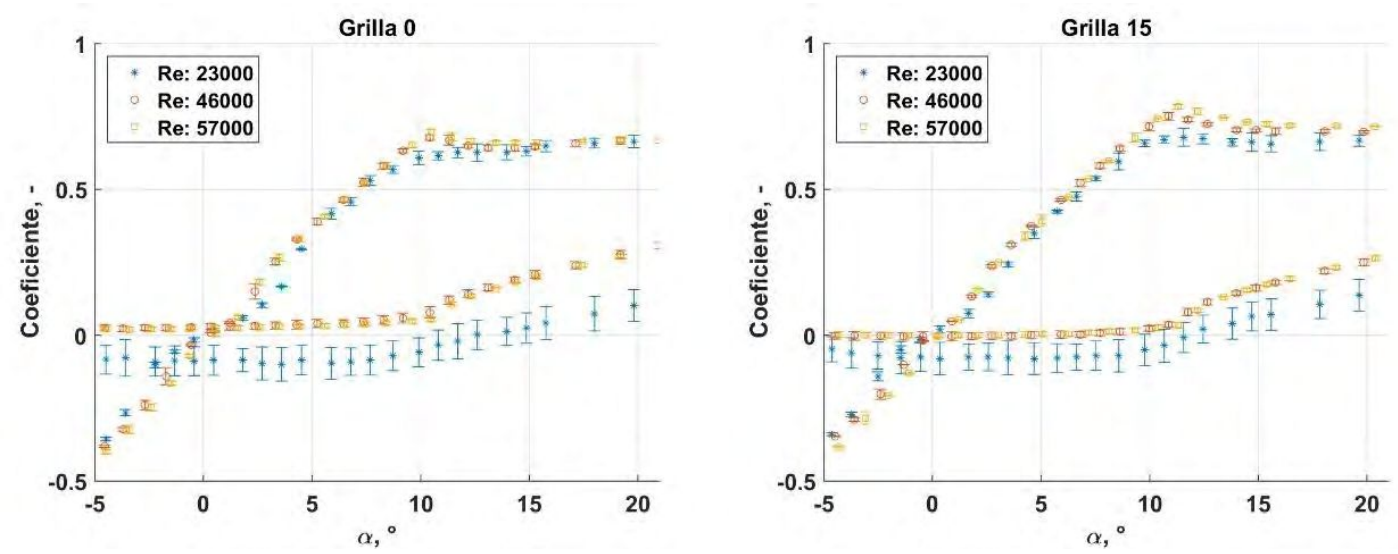

Figura 3.40. Comparación de influencia del Reynolds para cada turbulencia incidente.
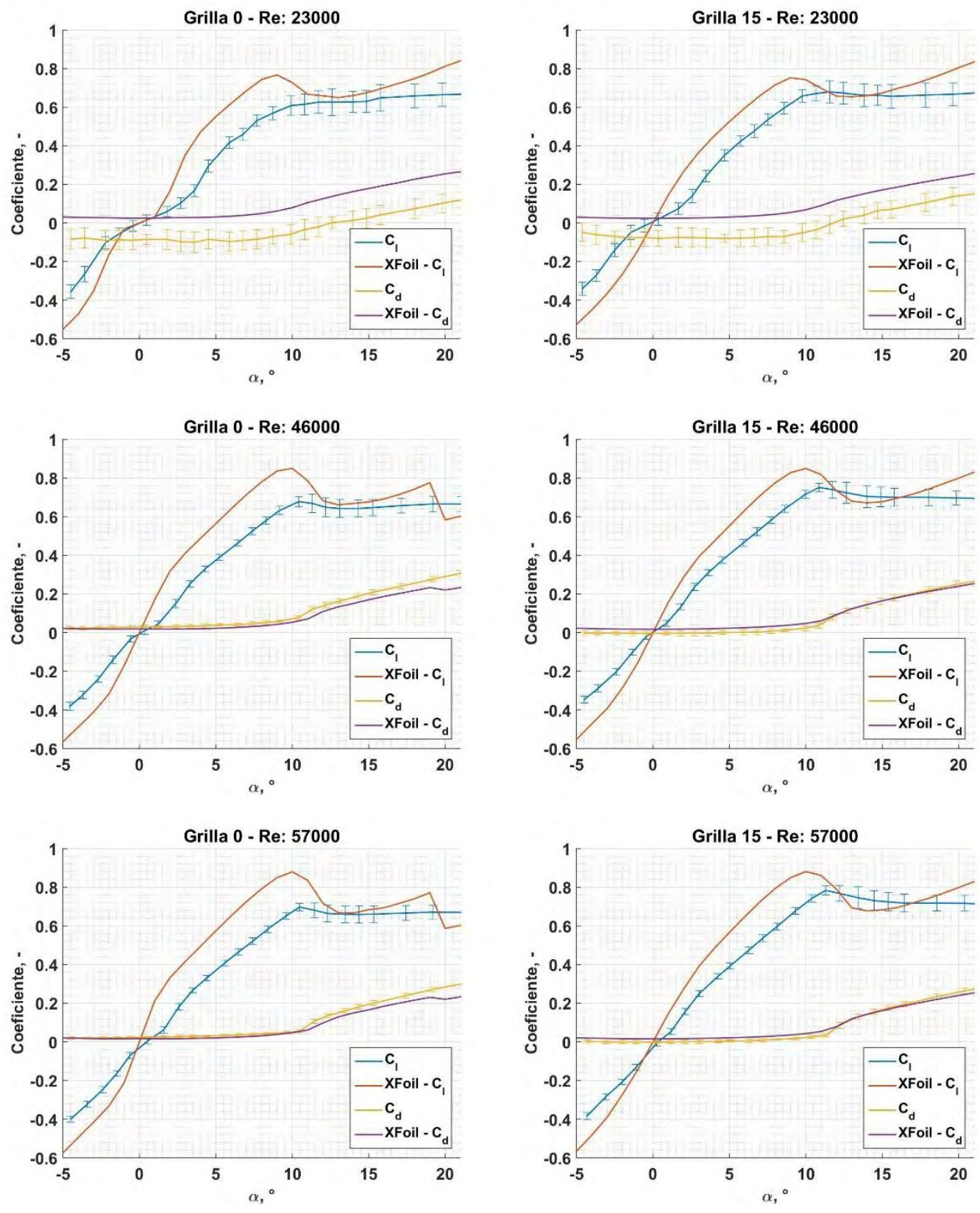

Figura 3.41. Comparación de resultados experimentales con simulaciones en XFoil, para los tres números de Reynolds ensayados y las dos condiciones de turbulencia incidente. 

de cabeceo"
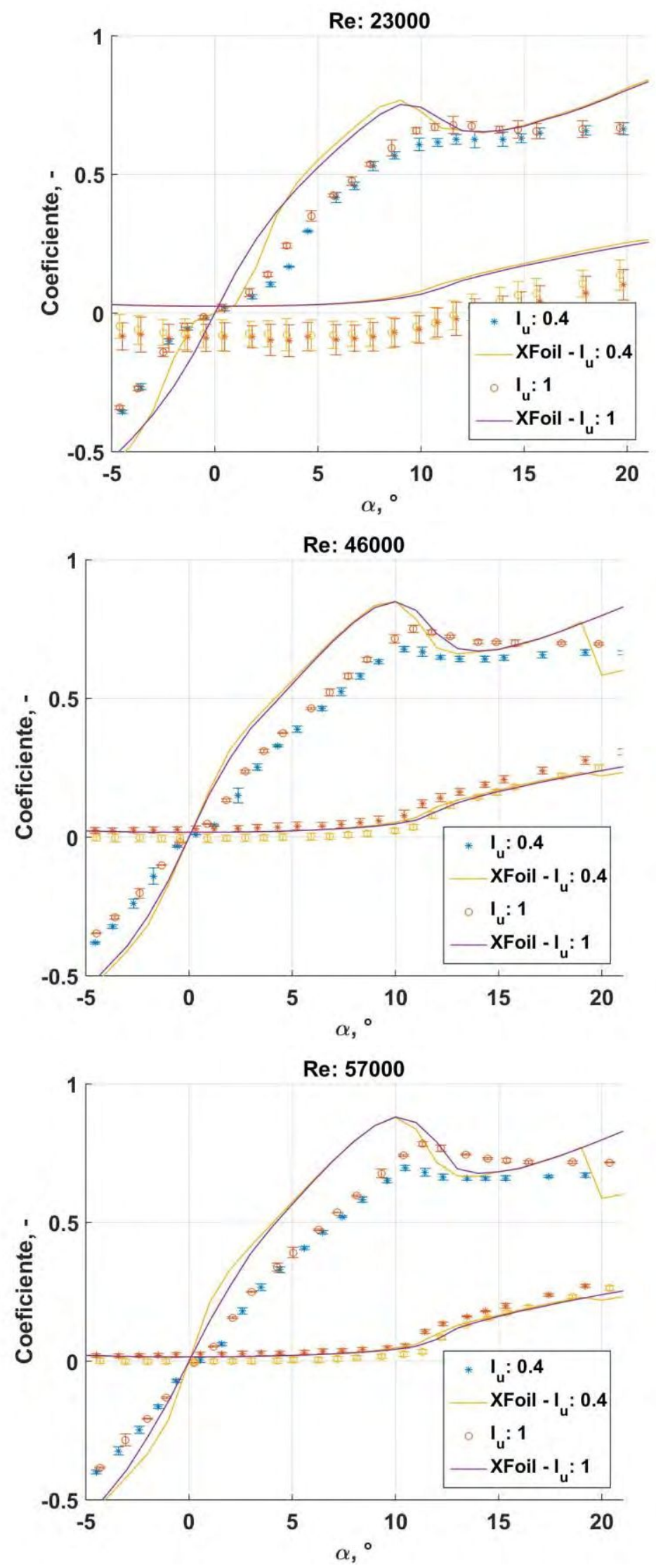

Figura 3.42. Comparación del coeficiente de sustentación a distintas intensidades de turbulencia para los tres números de Reynolds analizados. 
En la Tabla 3.6 se muestran los valores máximos de coeficiente de sustentación para cada nivel de turbulencia analizado, en los diferentes números de Reynolds. En todos los casos, al incrementarse la intensidad de turbulencia, se puede encontrar un valor mayor en el coeficiente de sustentación, que llega a incrementos del orden del 10\%.

Tabla 3.6. Coeficiente de sustentación máximo en función del Reynolds y la intensidad de turbulencia.

\begin{tabular}{l|rrr} 
Re & 23.000 & 46.000 & 57.000 \\
\hline Grilla 0 & 0,63 & 0,67 & 0,70 \\
Grilla 15 & 0,68 & 0,75 & 0,78
\end{tabular}

\subsubsection{Ensayos dinámicos: Movimiento continuo}

En esta sección nos concentraremos en la evolución de la fuerza de sustentación con movimiento continuo para el perfil alar ensayado. Nuevamente se analizará la evolución del coeficiente de sustentación a lo largo del tiempo, así como también su relación con el ángulo de ataque, para visualizar los efectos dinámicos respecto a la aerodinámica estacionaria mostrada en la sección anterior.

En primera instancia, se verifica la repetitividad de los ensayos, como se puede ver en la Figura 3.43 para el caso de mínima intensidad de turbulencia, Reynolds 23000 y una frecuencia reducida de 0,06 . En dicha figura se pueden observar cómo el valor medio de los 11 ciclos de cada iteración se comporta muy similarmente, sin presentar desvíos importantes. Además, si analizamos el comportamiento de cada ciclo, se puede ver una información similar, las cargas desarrolladas por cada ciclo se repite en los ciclos siguientes, Figura 3.44. Para verificar la repetitividad en los demás casos se puede consultar el Análisis de repetitividad de cargas aerodinámicas.

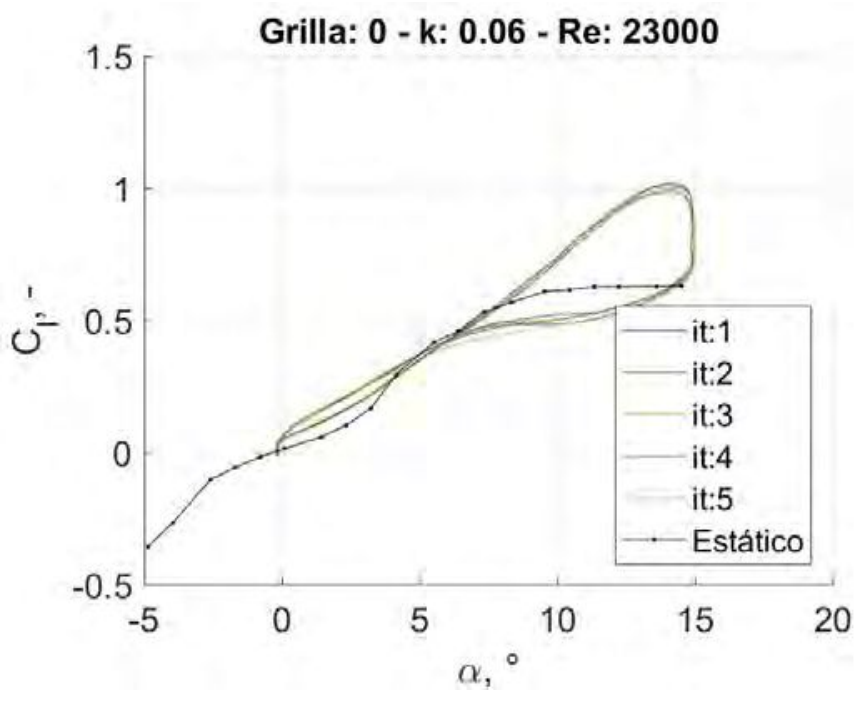

Figura 3.43. Cargas en función del ángulo de ataque para las distintas iteraciones realizadas. Caso de mínima turbulencia, Reynolds 23000 y máxima frecuencia reducida. 


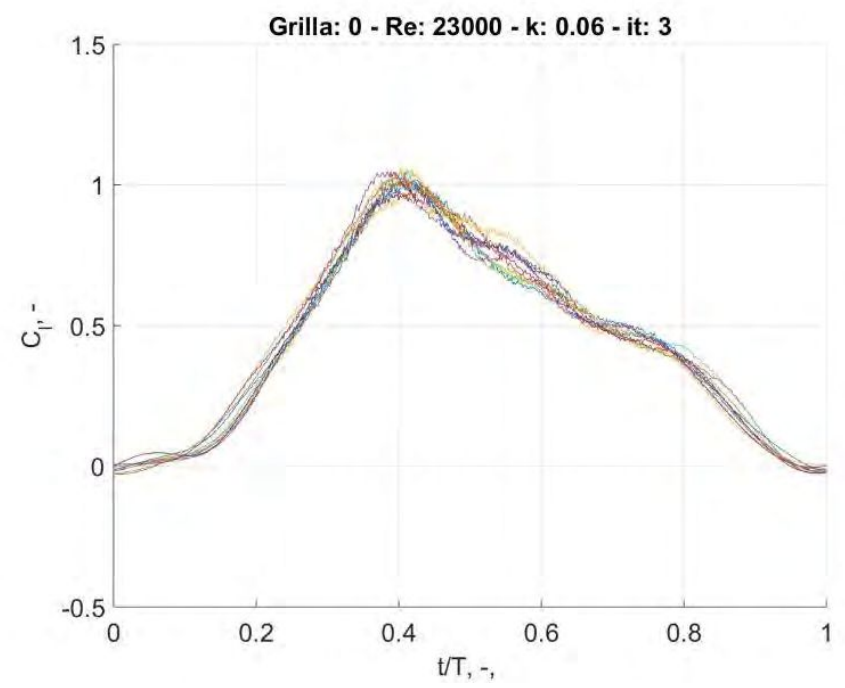

Figura 3.44. Comparación de las cargas en tiempo adimensional para los 11 ciclos en una iteración particular. Reynolds 23000 $\mathbf{y} \mathbf{k}=\mathbf{0 , 0 6}$.

El análisis de las cargas aerodinámicas se realizó en función de comparar la evolución respecto a dos variables: el tiempo adimensionalizado con la frecuencia de movimiento, y respecto al ángulo de ataque. En el caso del tiempo adimensionalizado, sirve para comparar respecto al movimiento el comportamiento de la carga, mientras que si lo analizamos respecto al ángulo de ataque, nos muestra claramente el desvío de la evolución de las cargas respecto al comportamiento estático, así como el efecto de histéresis.

$\mathrm{Al}$ analizar las gráficas en función del tiempo adimensionalizado, ver Figura 3.45, podemos observar como en el comienzo del movimiento ascendente las cargas están superpuestas, para ambas condiciones de turbulencia, pero para las distintas frecuencias de movimiento, se puede observar un retraso en la entrada en pérdida, así como también un incremento en el valor máximo de sustentación. Si comparamos el efecto de la intensidad de turbulencia (Grilla 0 vs. Grilla 15), podemos ver que al incrementar la intensidad de turbulencia obtenemos mayores valores en el coeficiente de sustentación, para todas las frecuencias reducidas, de igual manera que lo hallado en el caso estático. Por otro lado, al observar lo que sucede con las cargas en la carrera descendente $(\mathrm{t} / \mathrm{T}>0,5)$, se observa un comportamiento diferente para las frecuencias reducidas, perdiendo la superposición que se encontraba en el movimiento ascendente. Al incrementar la frecuencia reducida se puede observar una reducción en el coeficiente de sustentación durante la carrera descendente.

Si realizamos la visualización de los gráficos en función del ángulo de ataque, ver Figura 3.46, nuevamente se puede ver el incremento del coeficiente de sustentación máximo, así como el retraso de la entrada en pérdida. Además, se puede visualizar como decae el coeficiente de sustentación durante la corrida descendente, incluso pudiendo ser menores a los valores hallados en el ensayo estático. Si se comparan los resultados con la curva del ensayo estático, el efecto dinámico se puede representar como una "histéresis no simétrica". 

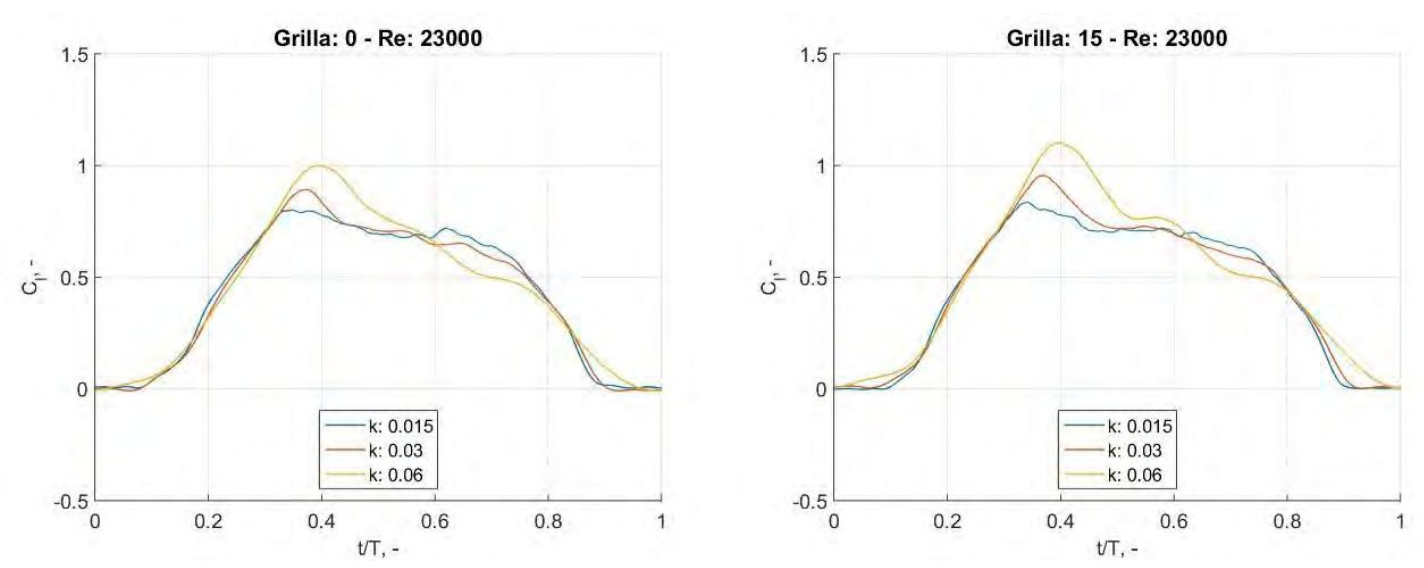

Figura 3.45. Comparación de las distintas frecuencias reducidas en tiempo adimensional para ambos niveles de turbulencia. Re $=\mathbf{2 3 0 0 0}$
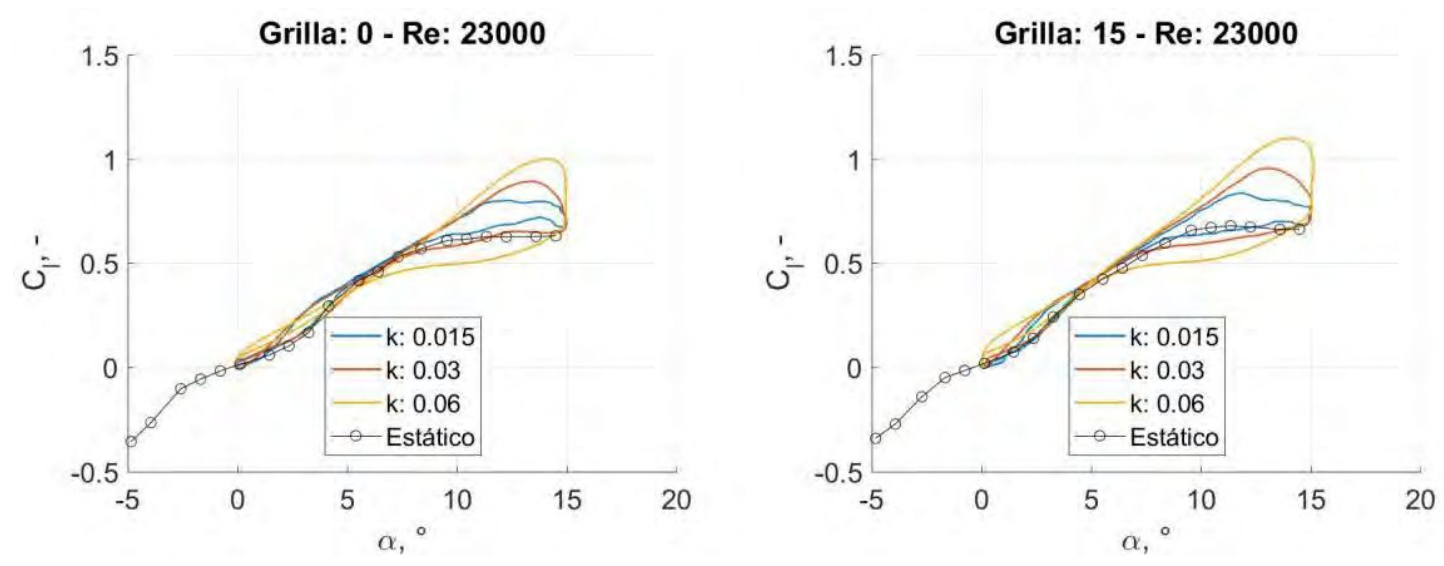

Figura 3.46. Comparación entre distintas frecuencias reducidas en A para ambas intensidades de turbulencia.

\subsection{Efecto de la turbulencia}

Para evidenciar el efecto de la turbulencia en la carga de sustentación, en la Figura 3.47 se graficaron los resultados medios de los diferentes ensayos, tanto en estático como dinámico para el Reynolds 23000 para las tres frecuencias reducidas analizadas. Allí se puede ver como con el incremento de la intensidad de turbulencia se generan mayores valores de sustentación, tanto para los casos dinámicos como estáticos. Para los ensayos dinámicos, dicha diferencia es más notoria en la carrera ascendente, pero no tan marcada en la zona descendente del movimiento. Un punto a denotar es que para el perfil aerodinámico, el número de Reynolds no generó un cambio en la influencia de turbulencia como si lo hizo en la placa plana. Es decir, para este perfil ensayado, el efecto de la turbulencia es notorio en todos los números de Reynolds, y no cambia su influencia con la velocidad de la corriente libre.

En la Tabla 3.7 se puede ver un resumen comparativo entre los valores más destacados del coeficiente de sustentación, para la condición estática y dinámica. A partir de la comparación, se puede observar cómo, al incrementar la frecuencia reducida se incrementa el valor máximo del coeficiente de sustentación. A su vez, se puede observar como la histéresis, salvo para el caso de menor frecuencia reducida, que presenta oscilaciones en la evolución de las cargas, no es tan afectada por el cambio de la turbulencia. Si se realiza el cociente entre la histéresis $\left(\Delta \mathrm{C}_{1}\right)$ y el coeficiente máximo de sustentación, se puede observar como la relación se mantiene aproximadamente constante con el cambio de la turbulencia, mientras que esta relación incrementa con el aumento de la frecuencia reducida. 
"Estudio aerodinámico experimental en flujo turbulento de bajo Reynolds sobre alas con movimiento de cabeceo"
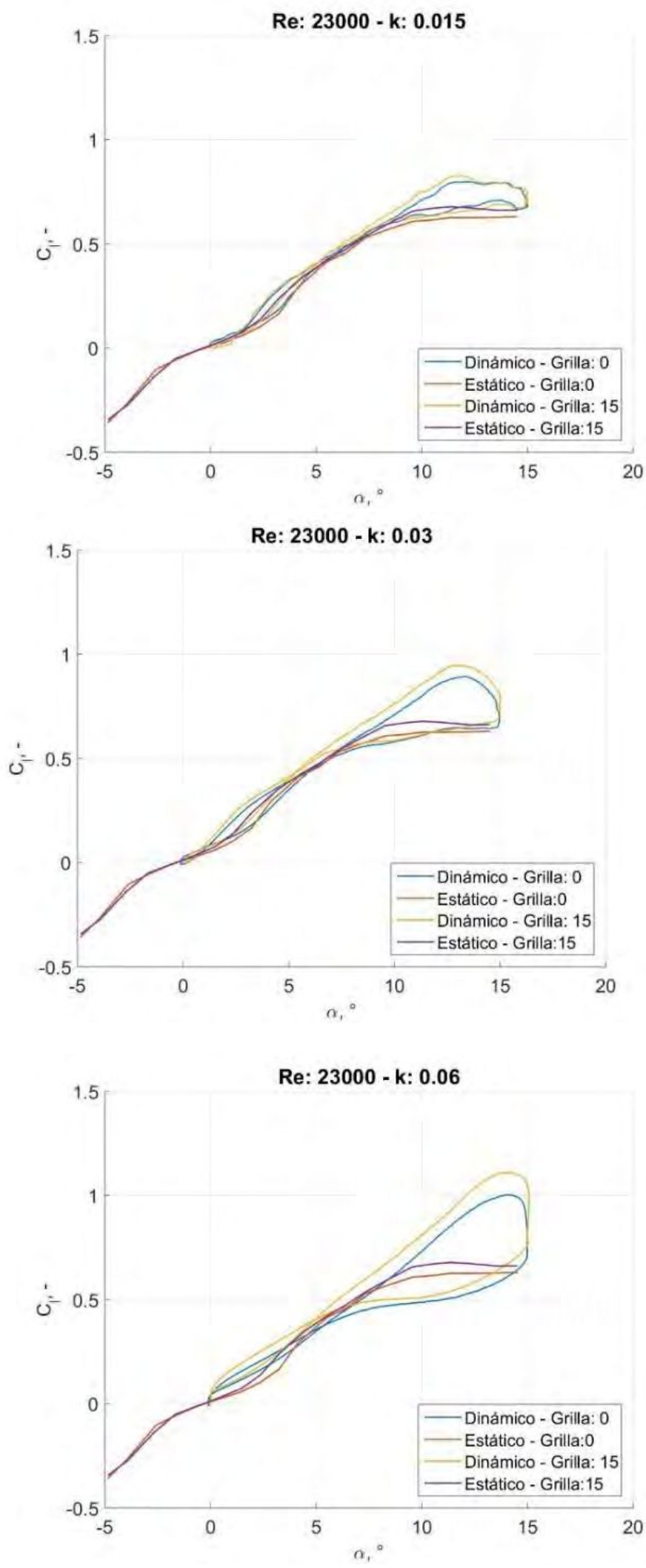

Figura 3.47. Comparación entre cargas en caso dinámica y estático versus $\alpha$ para ambas intensidades de turbulencia. Re: 23000 


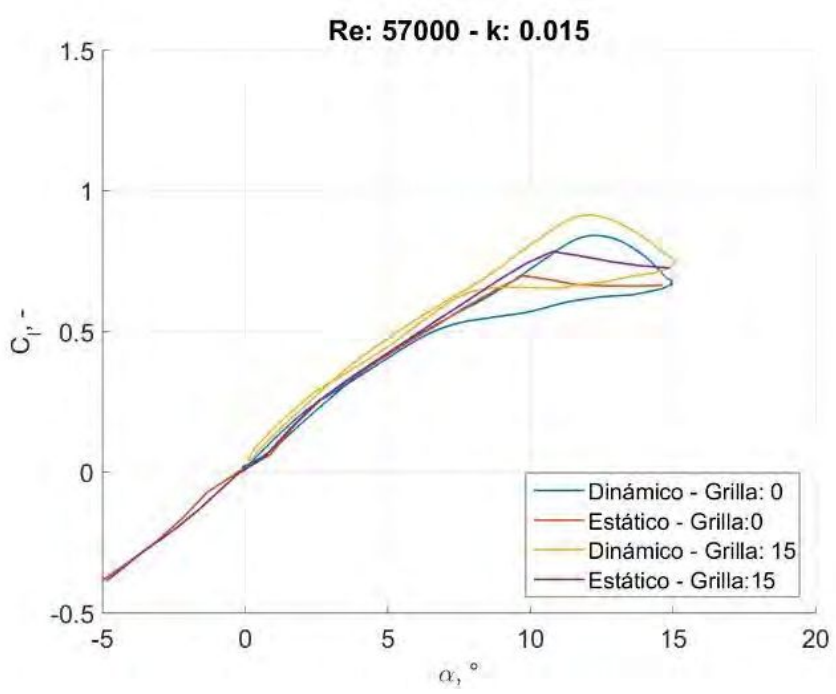

Figura 3.48. Comparación entre cargas en caso dinámica y estático versus $\alpha$ para ambas intensidades de turbulencia. Re: $\mathbf{5 7 0 0 0}$

Tabla 3.7. Valores de sustentación para Reynolds 23.000, estático y dinámico para perfil SD8020.

\begin{tabular}{l|c|cc|cc|cc|}
\multicolumn{1}{c}{ Estático } & \multicolumn{2}{c}{$k=0,015$} & \multicolumn{2}{c}{$k=0,03$} & \multicolumn{2}{c}{$k=0,06$} \\
\hline \multirow{3}{*}{ Grilla 0 } & $\mathrm{C}_{1}$ máximo & $\mathrm{C}_{1}$ máximo & $\Delta \mathrm{C}_{1}$ & $\mathrm{C}_{1}$ máximo & $\Delta \mathrm{C}_{1}$ & $\mathrm{C}_{1}$ máximo & $\Delta \mathrm{C}_{1}$ \\
Grilla 15 & 0,63 & 0,80 & 0,11 & 0,89 & 0,24 & 1,00 & 0,38 \\
\cline { 2 - 8 } & 0,66 & 0,83 & 0,17 & 0,95 & 0,3 & 1,10 & 0,43 \\
\hline
\end{tabular}

Tabla 3.8. Cociente entre histéresis $\left(\Delta \mathrm{C}_{l}\right)$ y el coeficiente máximo de sustentación.

\begin{tabular}{l|c|c|c}
\multicolumn{1}{c}{$k=0,015$} & $k=0,03$ & $k=0,06$ \\
\hline Grilla 0 & 0,137 & 0,269 & 0,380 \\
Grilla 15 & 0,204 & 0,315 & 0,391 \\
\cline { 2 - 4 }
\end{tabular}

\subsection{Presiones en la estela del modelo alar}

\subsubsection{Modelo de Placa Plana}

\subsubsection{Valores medios}

En esta sección se mostrarán los resultados hallados a partir de las mediciones realizadas con el rack de presiones sobre la estela. En particular es de interés el estudio de la pérdida de cantidad de movimiento, para la determinación del coeficiente de resistencia, así como determinación, mediante métodos estadísticos, de frecuencias predominantes en los desprendimientos de vórtices, y correlaciones entre mediciones realizadas a distintas distancias desde el borde de fuga.

En la Figura 3.49 se muestra, a modo de ejemplo, el perfil de velocidades hallado para una condición estática de ángulo de ataque. En dicha gráfica se compara los resultados hallados para las dos configuraciones de turbulencia de análisis en este trabajo. De igual manera que para las mediciones realizadas con cargas aerodinámicas, se puede observar que el incrementar la intensidad de turbulencia, produce una mayor pérdida de cantidad de movimiento, lo cual está directamente relacionado con el aumento del coeficiente de resistencia. En todos los casos analizados se encontraron los mimos resultados. En el eje de las abscisas se encuentra el valor del coeficiente de presiones $\left(\mathrm{C}_{\mathrm{P}}\right)$ que representa al valor de la resta entre la presión total y la presión 


\section{"Estudio aerodinámico experimental en flujo turbulento de bajo Reynolds sobre alas con movimiento de cabeceo"}

estática, dividido por la presión dinámica de referencia. En el eje de ordenadas se encuentra la distancia adimensionalizada medida desde el eje de rotación del ala, siendo valores positivos los valores por encima del ala, mientras que los negativos los ubicados por debajo del eje de rotación.

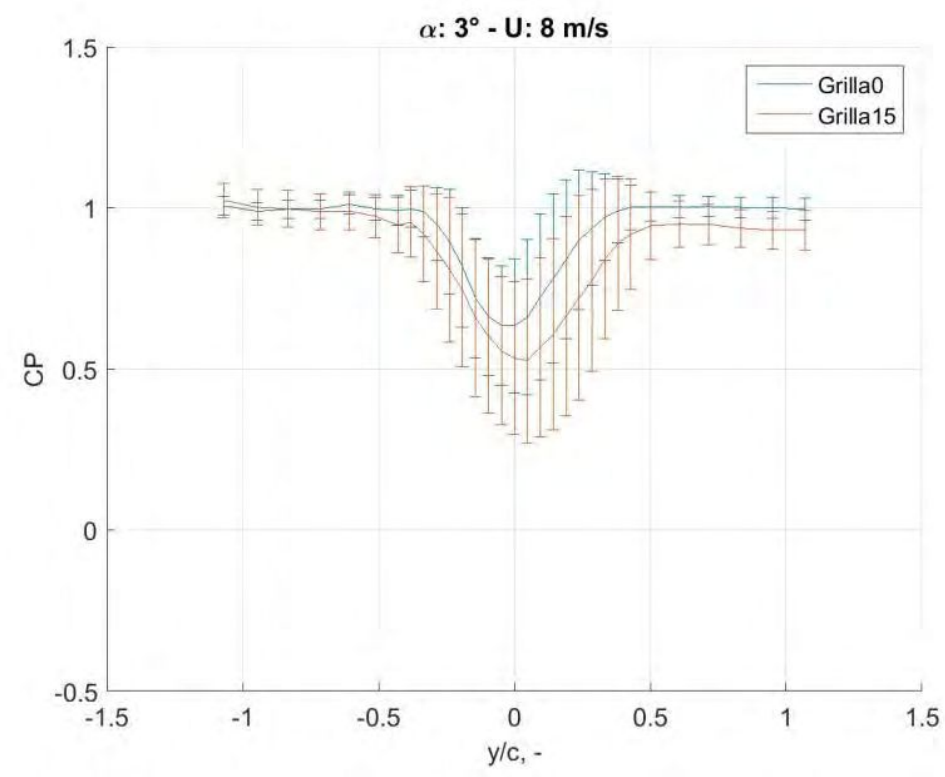

Figura 3.49. Coeficiente de Presiones en función de la distancia al eje de rotación. Ángulo de ataque fijo en $3^{\circ}$ y velocidad de corriente libre de $8 \mathrm{~m} / \mathrm{s}$. Las barras representan un desvío estándar.

A su vez, más allá de la utilidad de conocer la distribución de presiones medias, es de gran interés conocer el comportamiento temporal del campo de presiones, la presencia de vórtices, su espaciado temporal, la relación entre la frecuencia de movimiento del ala respecto a los vórtices en el fluido, su tamaño, entre otros. Para ello, se utilizaron técnicas de auto correlaciones y correlaciones cruzadas entre diferentes tomas, y correlaciones cruzadas para mediciones en las dos estaciones planteadas $(0,5 \mathrm{C}$ y $1 \mathrm{C})$. Además, se realizó un análisis en frecuencias y usando transformaciones de wavelet, para tener información en el espacio temporal y de frecuencias en simultáneo. Los resultados de dichos análisis se presentarán a continuación, para ambos niveles de turbulencia y los tres números de Reynolds ensayados, para cada frecuencia reducida.

\subsubsection{Correlaciones cruzadas en la estación 0,5C}

En primera instancia, se realizó la correlación cruzada entre las tomas del rack de presiones, con las mediciones correspondientes a una distancia de media cuerda aguas abajo. Para ello se considera como presión de referencia a la presión medida por el sensor localizado a la misma altura que el eje de rotación. En las figuras se puede observar en el eje de las ordenadas, el desplazamiento de las señales, respecto a la señal de referencia, en tiempo adimensionalizado con la frecuencia de movimiento; en el eje de las abscisas, la ubicación de las tomas de presión, adimensionalizado con la cuerda, y en colores, el valor del coeficiente de correlación, siendo su valor máximo de 1 correspondiente con la correlación de dos señales idénticas y -1 para señales idénticamente opuestas. Los contornos fueron coloreados con un paso de 0,2 en el coeficiente de correlación, y los valores entre - $-0,2$ y 0,2 fueron unificados a un único color. Como referencia, se muestra con una línea punteada negra el valor correspondiente a $\pm 0,25 \mathrm{C}$ de ubicación de los sensores, y en línea punteada roja la ubicación del eje de rotación, para la determinación del tamaño de los vórtices y su localización.

Desde la Figura 3.50 a la Figura 3.55 se puede observar los resultados hallados para las correlaciones cruzadas entre el sensor localizado a la altura del eje de rotación con los demás sensores. A partir de dichos resultados se puede apreciar el tamaño de la estela que genera el perfil oscilando a distintas frecuencias reducidas 
y distintos números de Reynolds. En las figuras, para todos los números de Reynolds, considerando el caso de mínima turbulencia, se puede observar cómo, considerando el valor de correlación de 0,5, los vórtices tienen un tamaño equivalente a media cuerda $(0,25 \mathrm{C}$ hacia arriba y lo mismo hacia abajo), mientras que, si consideramos cualquier valor positivo de correlación, dicho tamaño alcanza un valor cercano al valor de $0,8 \sim 0,9$ cuerdas. Por otro lado, al analizar la influencia del aumento de la turbulencia, se puede observar como la correlación disminuye rápidamente al desplazar la señal temporalmente, aunque se mantiene el tamaño de los vórtices. Este efecto se va mitigando a medida que se aumenta el número de Reynolds, observándose a un Reynolds de 57.000 un comportamiento muy similar al de mínima turbulencia. Si el análisis se concentra sobre el primer ciclo, el tamaño del vórtice posee un tamaño similar a los encontrados en condiciones de mínima turbulencia, pero, al analizar la correlación disminuye como es de esperar, producto de las propiedades de disipación viscosa de la turbulencia, mientras que para una condición de mínima intensidad de turbulencia es más homogéneo el tamaño de los desprendimientos a lo largo del tiempo.
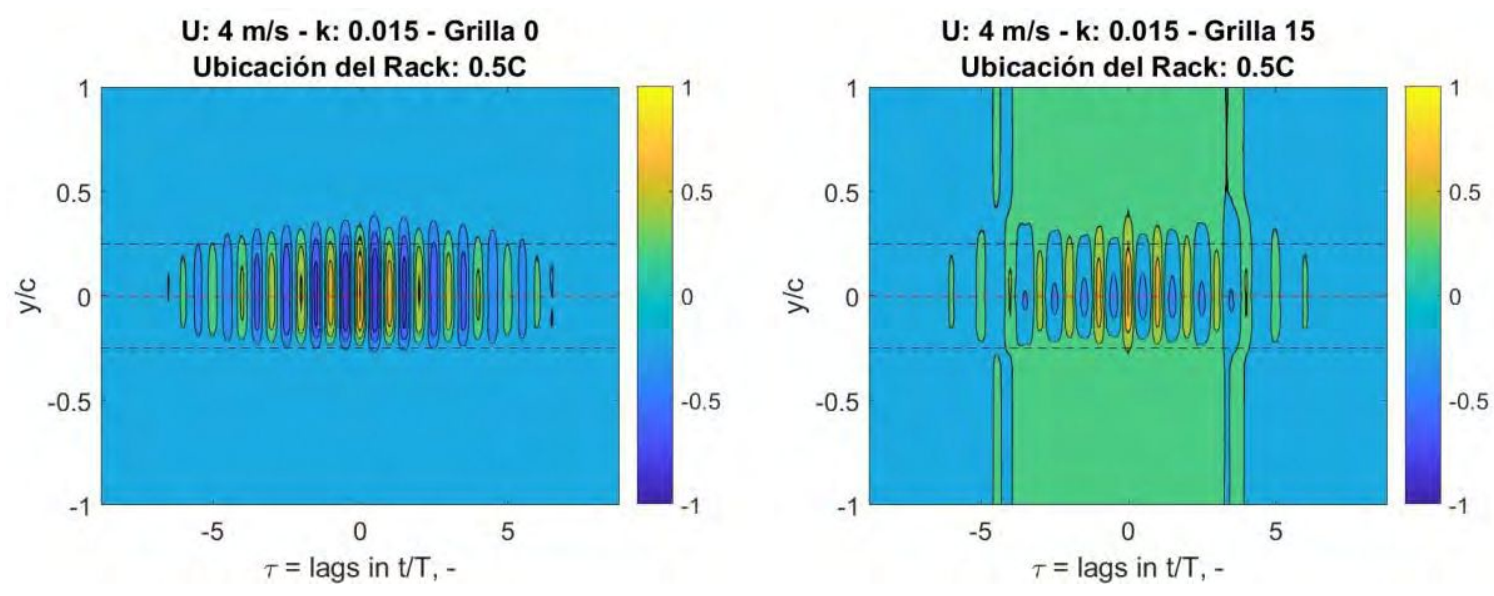

Figura 3.50. Correlación cruzada entre el sensor localizado a la altura del eje de rotación y los demás sensores, para el caso de Reynolds 23.000, $\mathrm{k}=\mathbf{0 , 0 1 5}$ y ambas intensidades de turbulencia. Estación 0,5C.
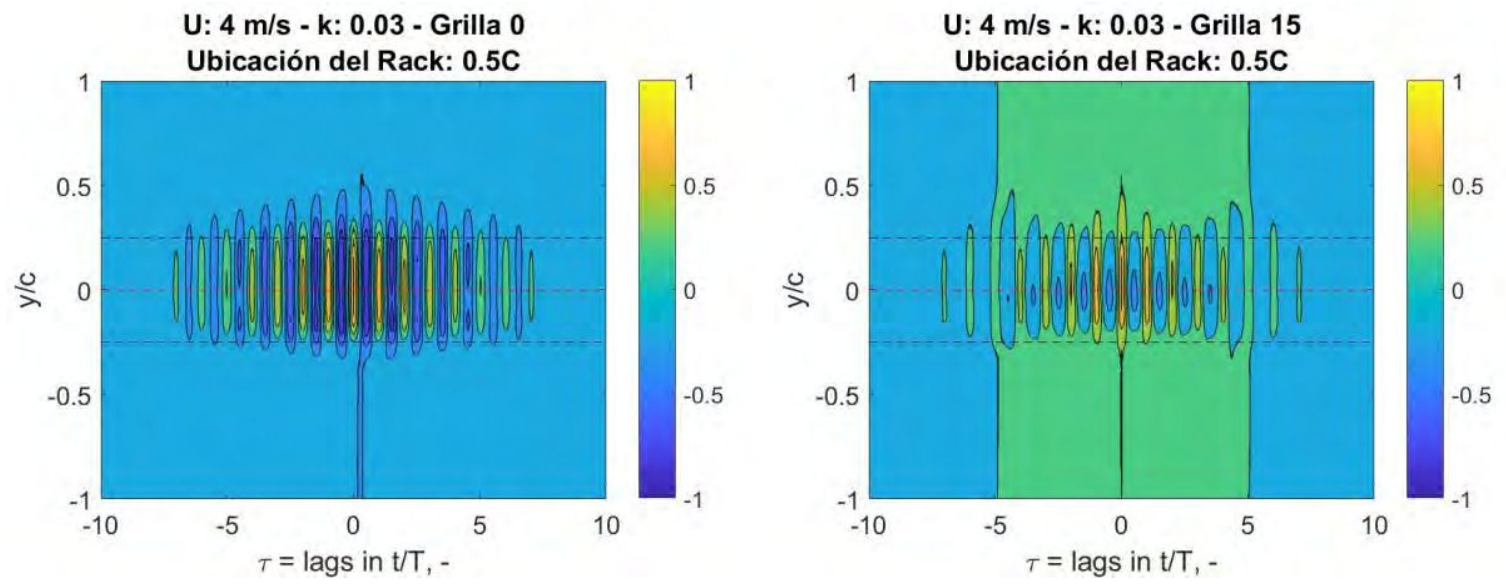

Figura 3.51. Correlación cruzada entre el sensor localizado a la altura del eje de rotación y los demás sensores, para el caso de Reynolds 23.000, $\mathrm{k}=0,03$ y ambas intensidades de turbulencia. Estación 0,5C. 

de cabeceo"
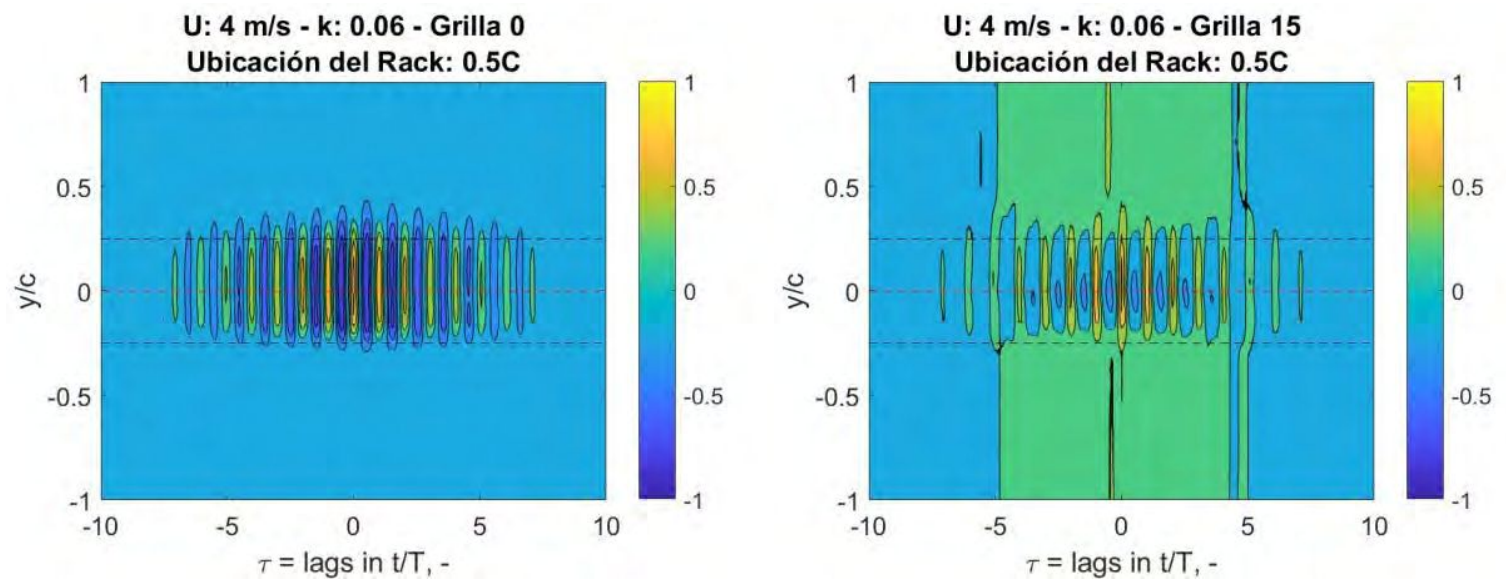

Figura 3.52. Correlación cruzada entre el sensor localizado a la altura del eje de rotación y los demás sensores, para el caso de Reynolds 23.000, $\mathrm{k}=0,06$ y ambas intensidades de turbulencia. Estación 0,5C.
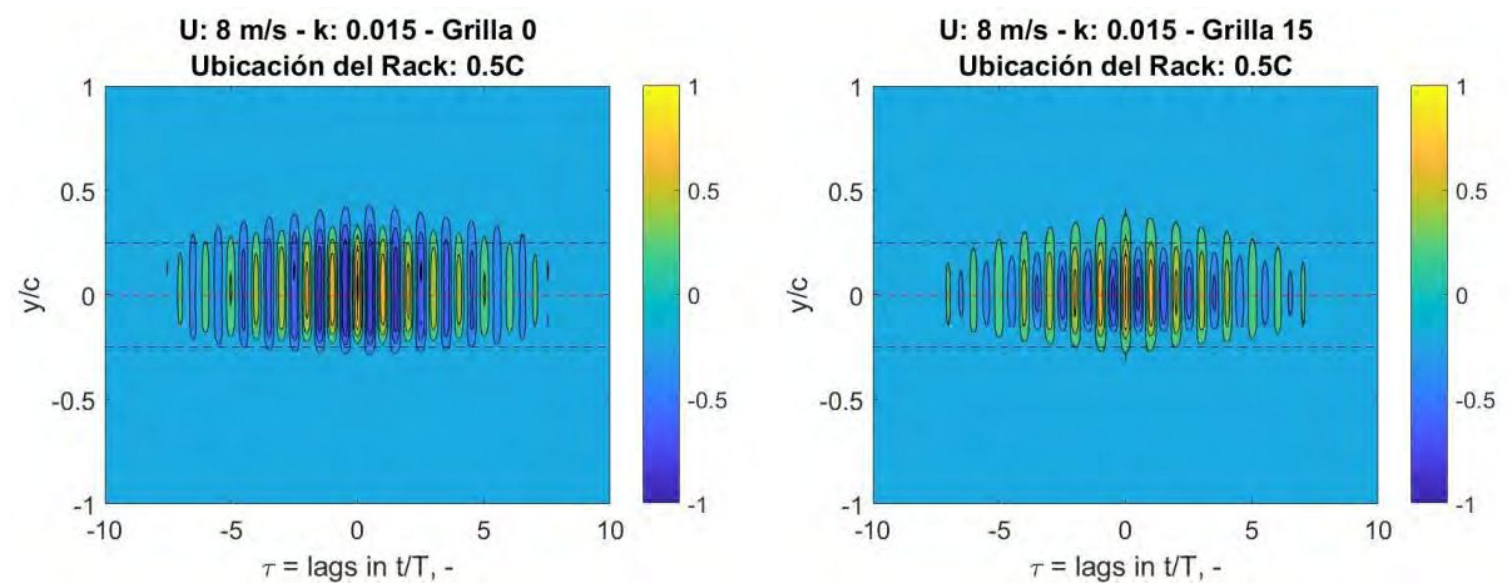

Figura 3.53. Correlación cruzada entre el sensor localizado a la altura del eje de rotación y los demás sensores, para el caso de Reynolds 46.000, $\mathrm{k}=0,015$ y ambas intensidades de turbulencia. Estación 0,5C.
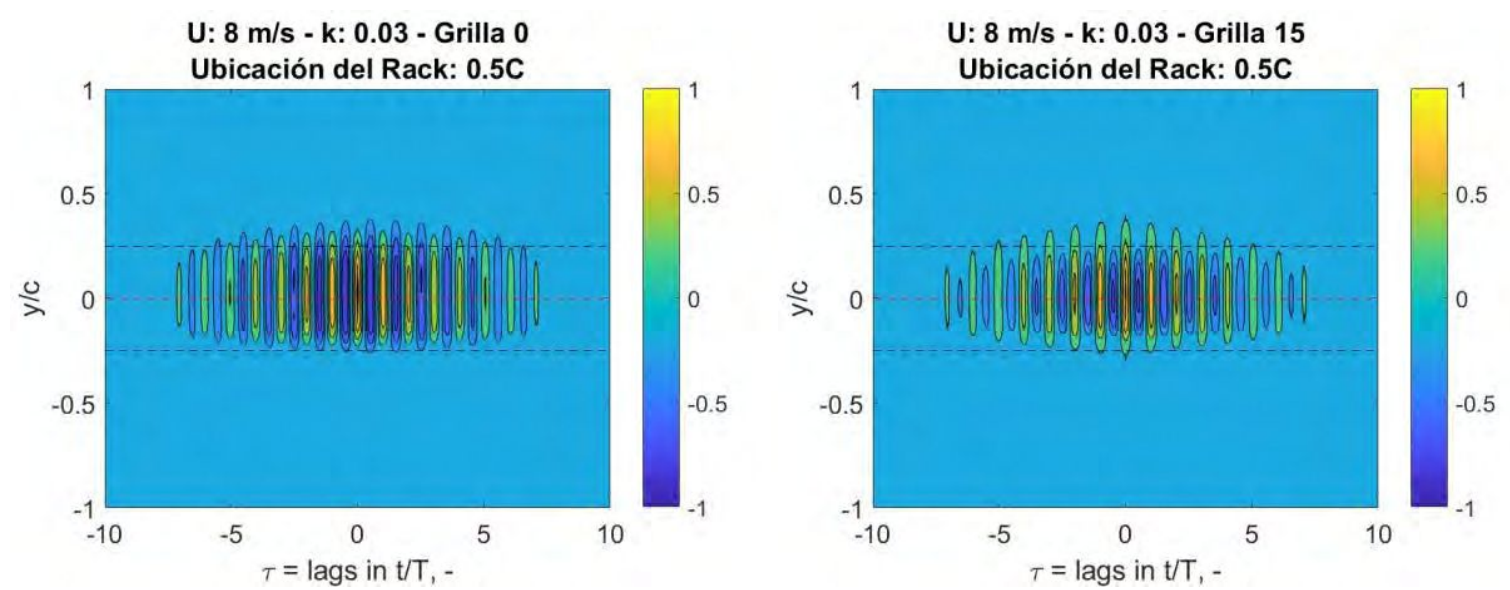

Figura 3.54. Correlación cruzada entre el sensor localizado a la altura del eje de rotación y los demás sensores, para el caso de Reynolds 46.000, $\mathrm{k}=0,03$ y ambas intensidades de turbulencia. Estación 0,5C. 

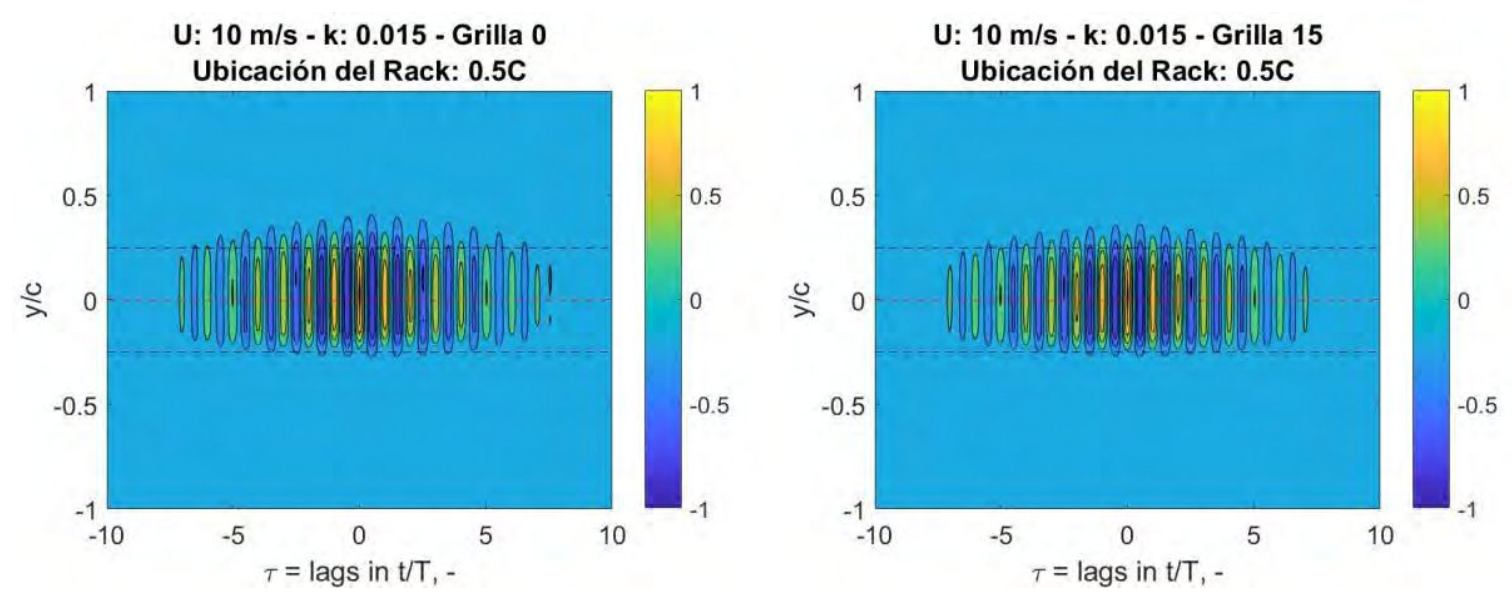

Figura 3.55. Correlación cruzada entre el sensor localizado a la altura del eje de rotación y los demás sensores, para el caso de Reynolds 57.000, $\mathrm{k}=$ 0,015 y ambas intensidades de turbulencia. Estación 0,5C.

\subsubsection{Correlaciones cruzadas en la estación 1C}

Al igual que para la estación ubicada a media cuerda de distancia, se realizó el mismo análisis de correlación cruzada, esperando encontrar menores valores de correlación dado que nos encontramos más alejados del perfil. Desde la Figura 3.56 hasta la Figura 3.61, se pueden observar los resultados. En dichas figuras se puede observar como el patrón de vórtices definidos y con la periodicidad esperada, dominada por la frecuencia del movimiento del ala, empieza a desaparecer y no está tan definida. Además, se empiezan a encontrar valores altos de correlación con las zonas por fuera de la estela, marcando que los vórtices han sido difuminados en el campo del flujo. Otro punto que destacar es que las zonas que indicarían presencia de vórtices se encuentran desplazadas del centro hacia arriba, en comparación con lo encontrado en los resultados a una distancia de media cuerda por detrás del perfil. Una vez más, al aumentar la intensidad de turbulencia, sobre todo a valores mayores de número de Reynolds, los efectos de correlación se encuentran difuminados y menos periódicos.
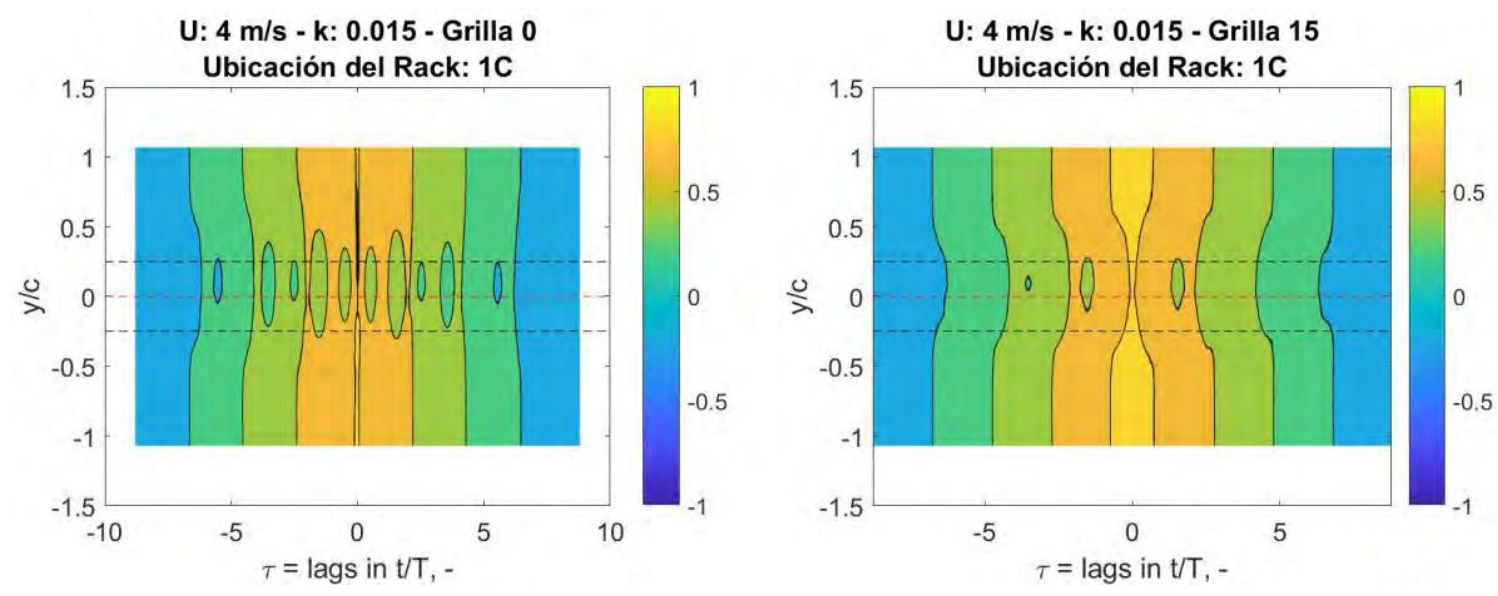

Figura 3.56. Correlación cruzada entre el sensor localizado a la altura del eje de rotación y los demás sensores, para el caso de Reynolds 23.000, $\mathrm{k}=0,015$ y ambas intensidades de turbulencia. Estación 1C. 

de cabeceo"
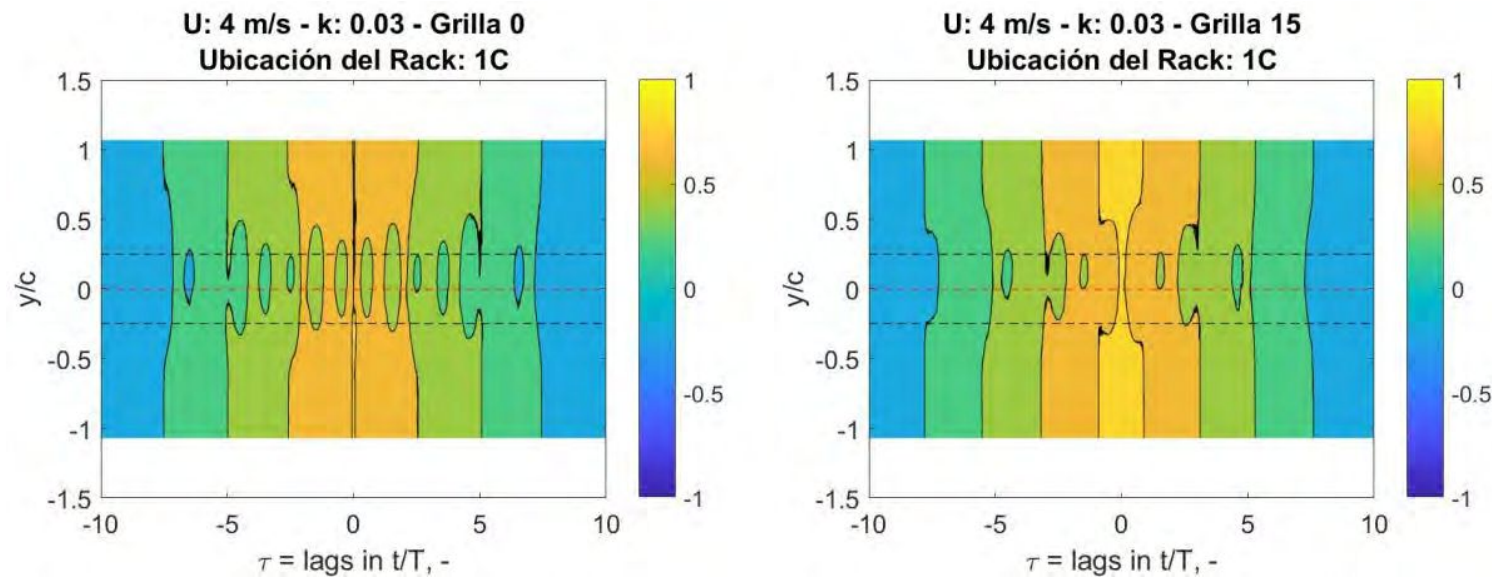

Figura 3.57. Correlación cruzada entre el sensor localizado a la altura del eje de rotación y los demás sensores, para el caso de Reynolds 23.000, $\mathrm{k}=0,03$ y ambas intensidades de turbulencia. Estación 1C.
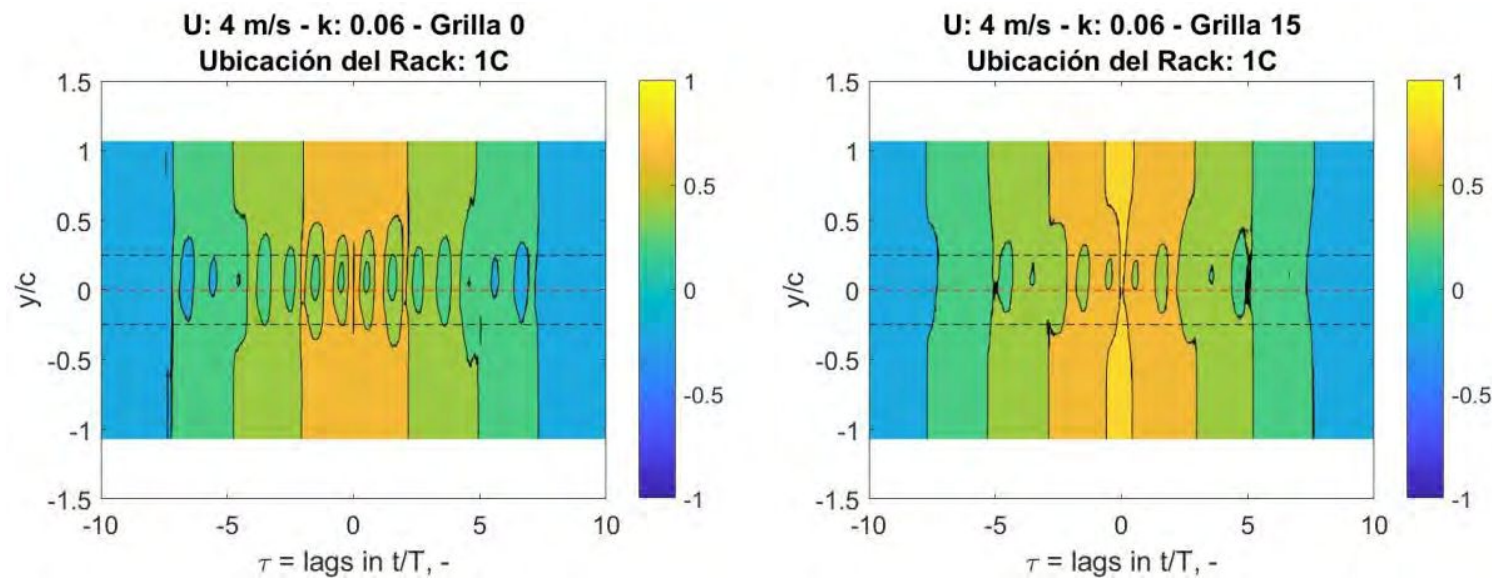

Figura 3.58. Correlación cruzada entre el sensor localizado a la altura del eje de rotación y los demás sensores, para el caso de Reynolds 23.000, $\mathrm{k}=0,06 \mathrm{y}$ ambas intensidades de turbulencia. Estación $1 \mathrm{C}$.
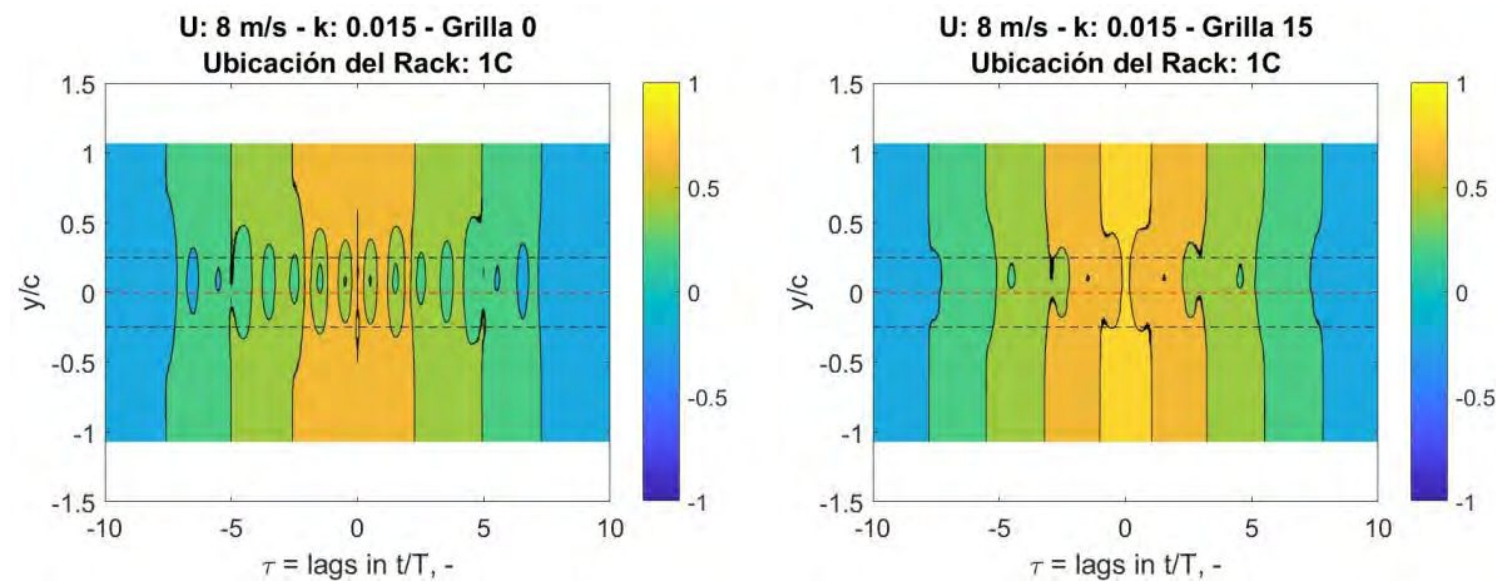

Figura 3.59. Correlación cruzada entre el sensor localizado a la altura del eje de rotación y los demás sensores, para el caso de Reynolds 46.000, $\mathrm{k}=0,015$ y ambas intensidades de turbulencia. Estación 1C. 

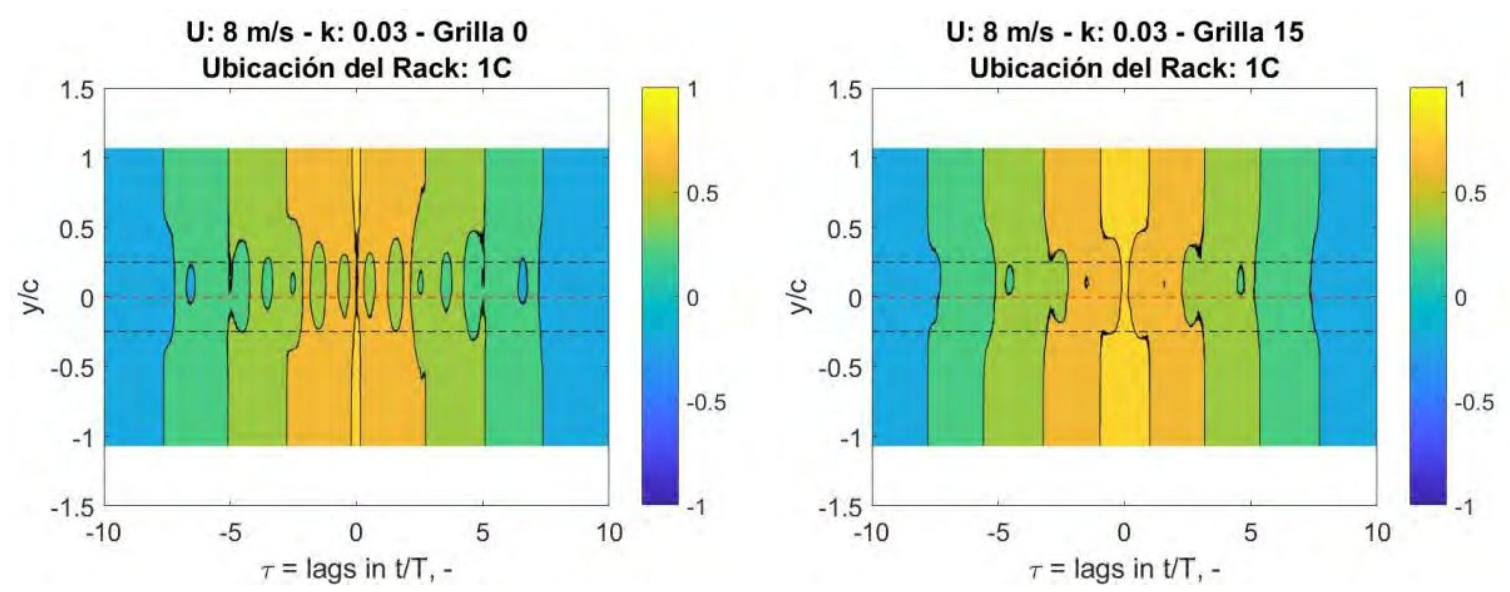

Figura 3.60. Correlación cruzada entre el sensor localizado a la altura del eje de rotación y los demás sensores, para el caso de Reynolds 46.000, $\mathrm{k}=0,03$ y ambas intensidades de turbulencia. Estación $1 \mathrm{C}$.
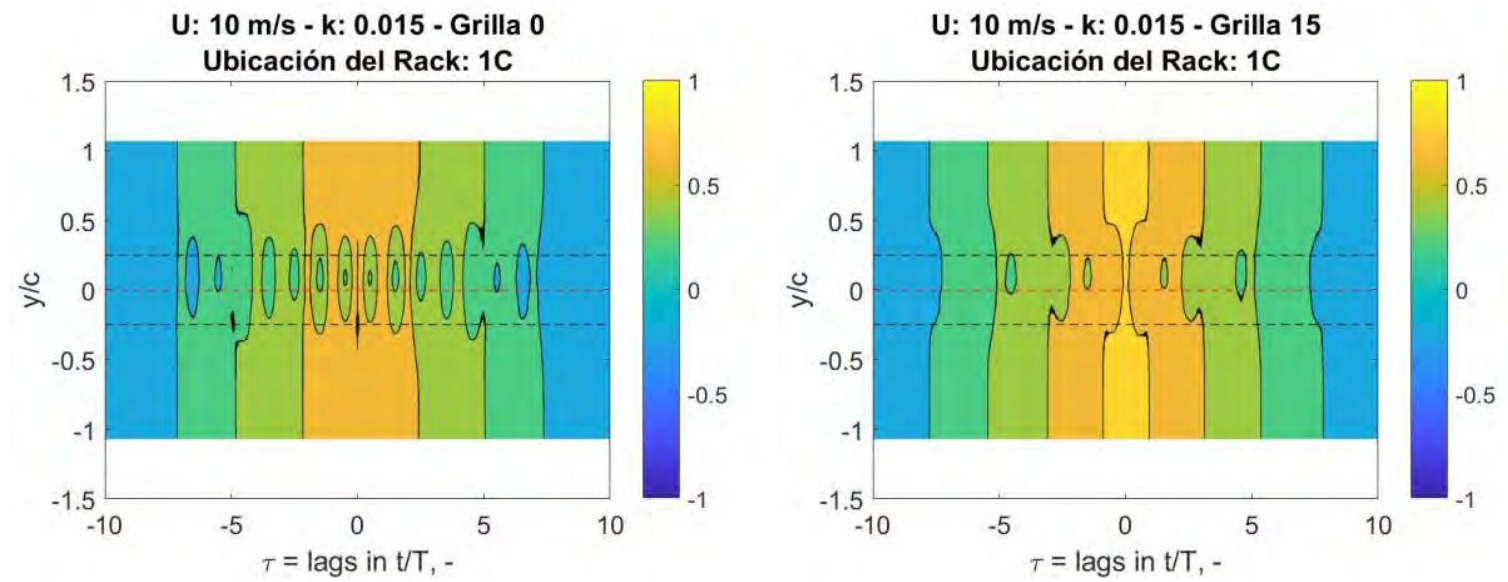

Figura 3.61. Correlación cruzada entre el sensor localizado a la altura del eje de rotación y los demás sensores, para el caso de Reynolds 57.000, $\mathrm{k}=0,015$ y ambas intensidades de turbulencia. Estación 1C.

\subsubsection{Correlación cruzada entre estación 0,5C y $1 C$}

Para analizar las correlaciones cruzadas, pero realizadas entre las dos diferentes estaciones de medición, se tomó como señal de referencia la localizada en la altura del eje de rotación de la estación ubicada en la estación alejada media cuerda del ala, y se la comparó con las presiones en la estación localizada a una cuerda de distancia. De esta forma, podemos analizar el desplazamiento de los vórtices, si hay desfasaje temporal y si los mismos se desplazan verticalmente. Para mayor claridad, solo se muestran los desfasajes temporales positivos, y se colocaron líneas punteadas, como referencia, en los valores de y/c de 0 (altura del eje de rotación, en color rojo) y 0,1 (en color negro), para marcar el desplazamiento de los vórtices.

Desde la Figura 3.62 hasta la Figura 3.67, en la columna izquierda se representan los resultados para mínima intensidad de turbulencia, mientras que en la columna derecha, los valores para máxima intensidad de turbulencia ensayada. En los gráficos se puede observar como para todos los casos el centro de los vórtices, denotado por el valor de máxima correlación, se ha desplazado un valor de 0,1 cuerdas, hacia arriba (lado de succión del ala) al trasladarse aguas abajo media cuerda. A su vez, la periodicidad sigue marcada por la frecuencia del movimiento del ala, sin encontrarse un desfasaje en dicho sentido. Nuevamente, al analizar el efecto de la turbulencia, encontramos menores valores de correlación cuando se aumenta la turbulencia, como se esperaba. 

de cabeceo"
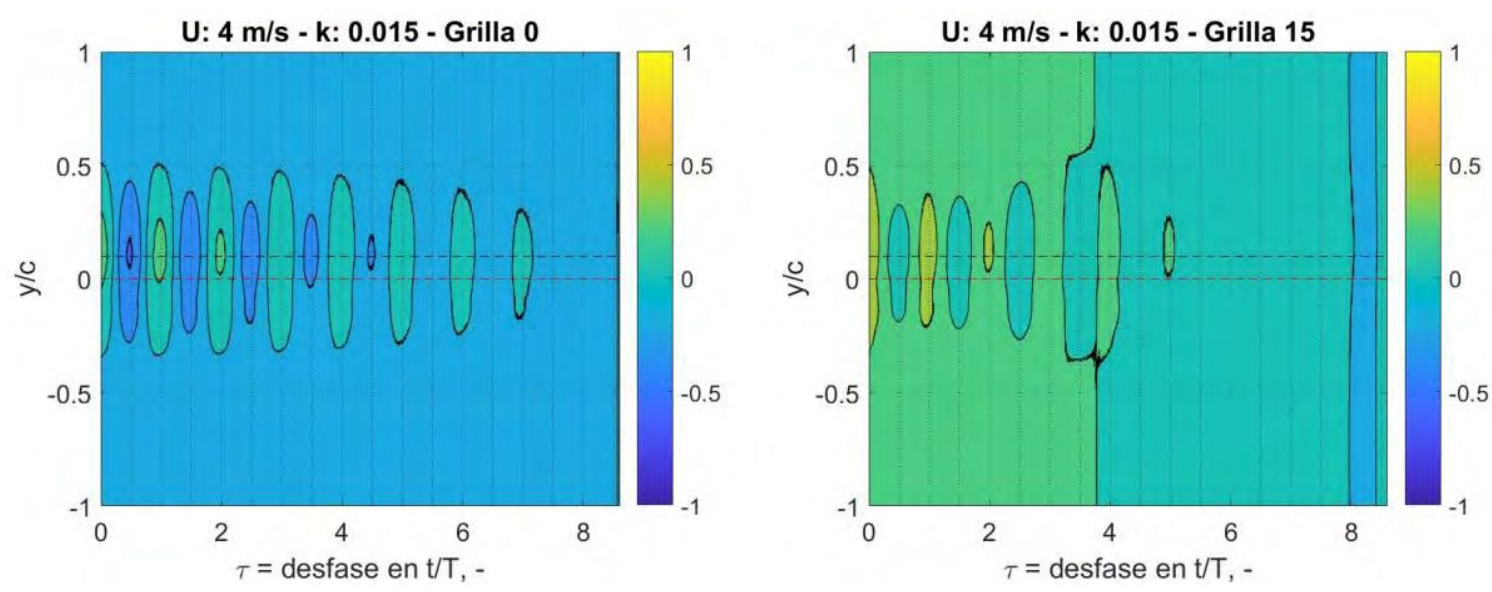

Figura 3.62. Correlación cruzada entre el sensor localizado a la altura del eje de rotación de la estación $0,5 \mathrm{C}$ y los sensores de la estación 1C, para el caso de Reynolds $23.000, \mathrm{k}=0,015 \mathrm{y}$ ambas intensidades de turbulencia.
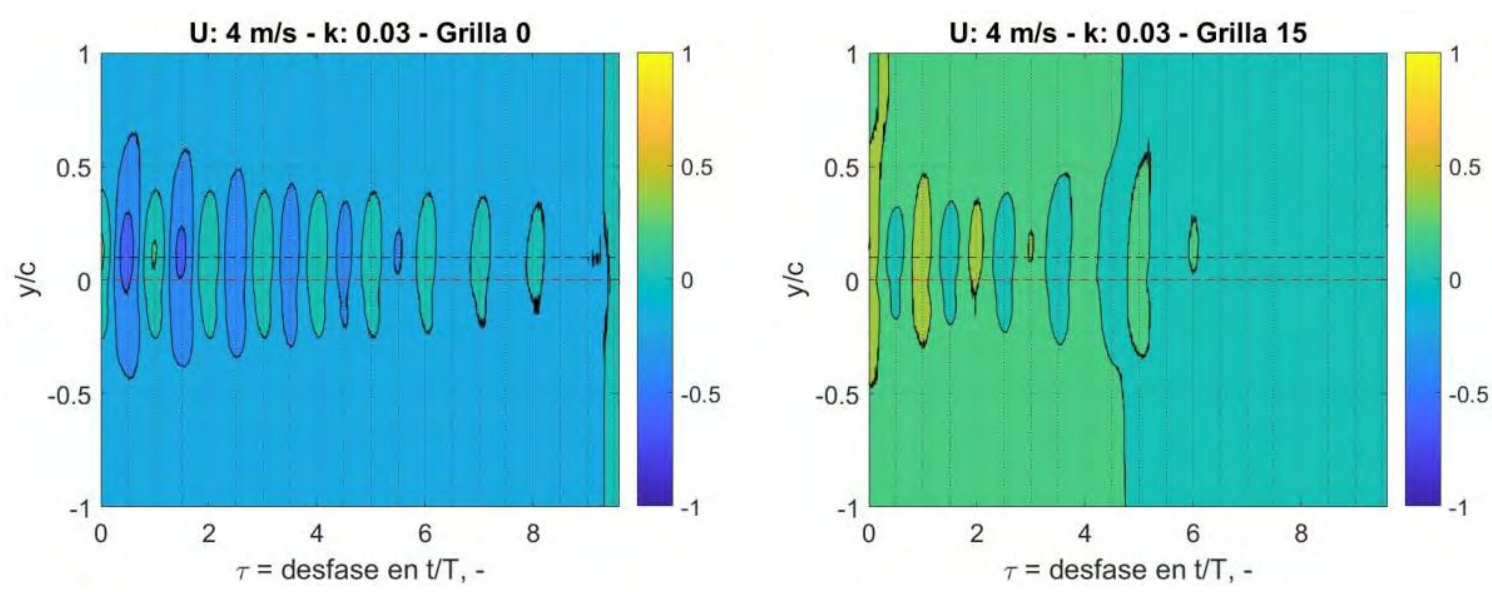

Figura 3.63. Correlación cruzada entre el sensor localizado a la altura del eje de rotación de la estación $0,5 \mathrm{C}$ y los sensores de la estación $1 \mathrm{C}$, para el caso de Reynolds $23.000, \mathrm{k}=0,03$ y ambas intensidades de turbulencia.
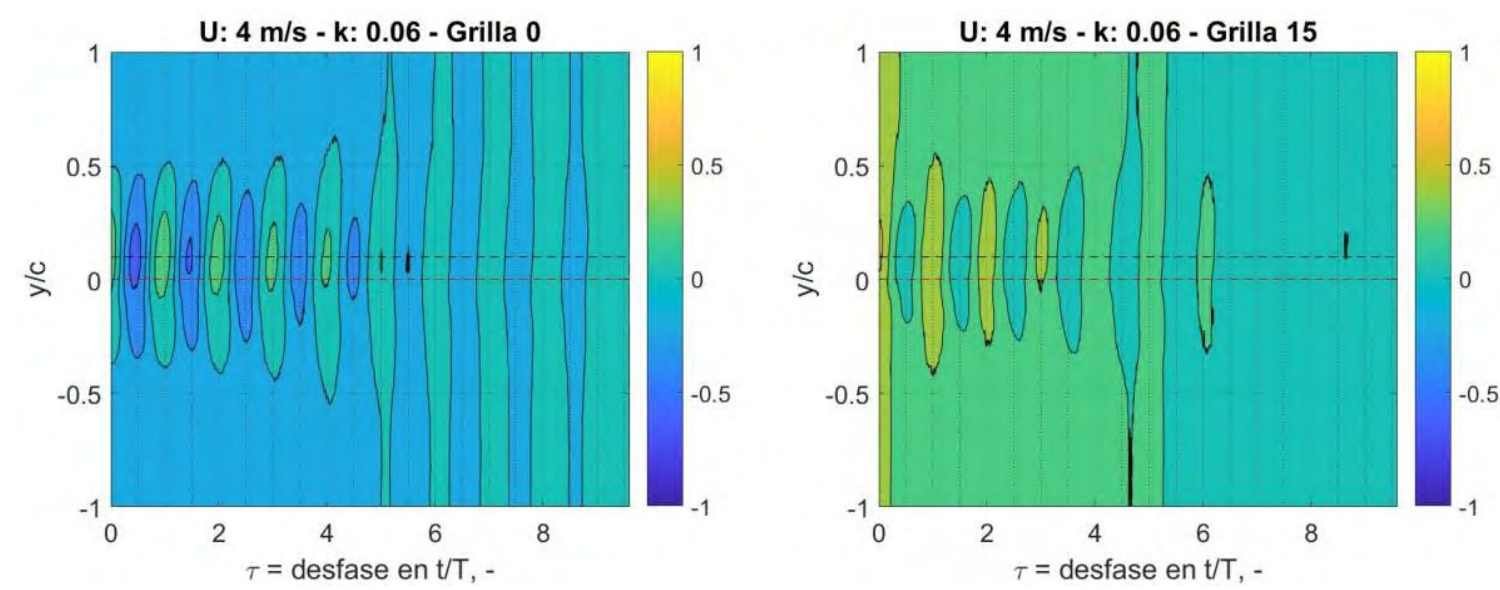

Figura 3.64. Correlación cruzada entre el sensor localizado a la altura del eje de rotación de la estación 0,5C y los sensores de la estación 1C, para el caso de Reynolds $23.000, k=0,06$ y ambas intensidades de turbulencia. 


\section{“Resultados y Análisis”}
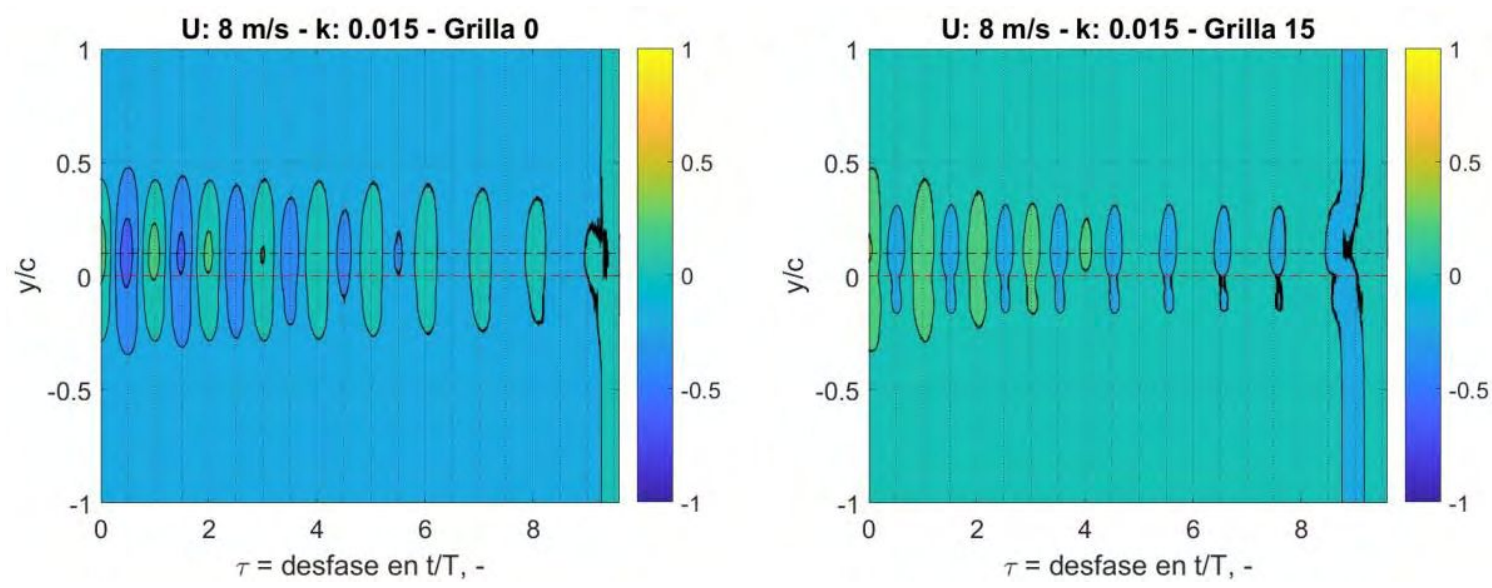

Figura 3.65. Correlación cruzada entre el sensor localizado a la altura del eje de rotación de la estación 0,5C y los sensores de la estación $1 \mathrm{C}$, para el caso de Reynolds $46.000, \mathrm{k}=0,015 \mathrm{y}$ ambas intensidades de turbulencia.
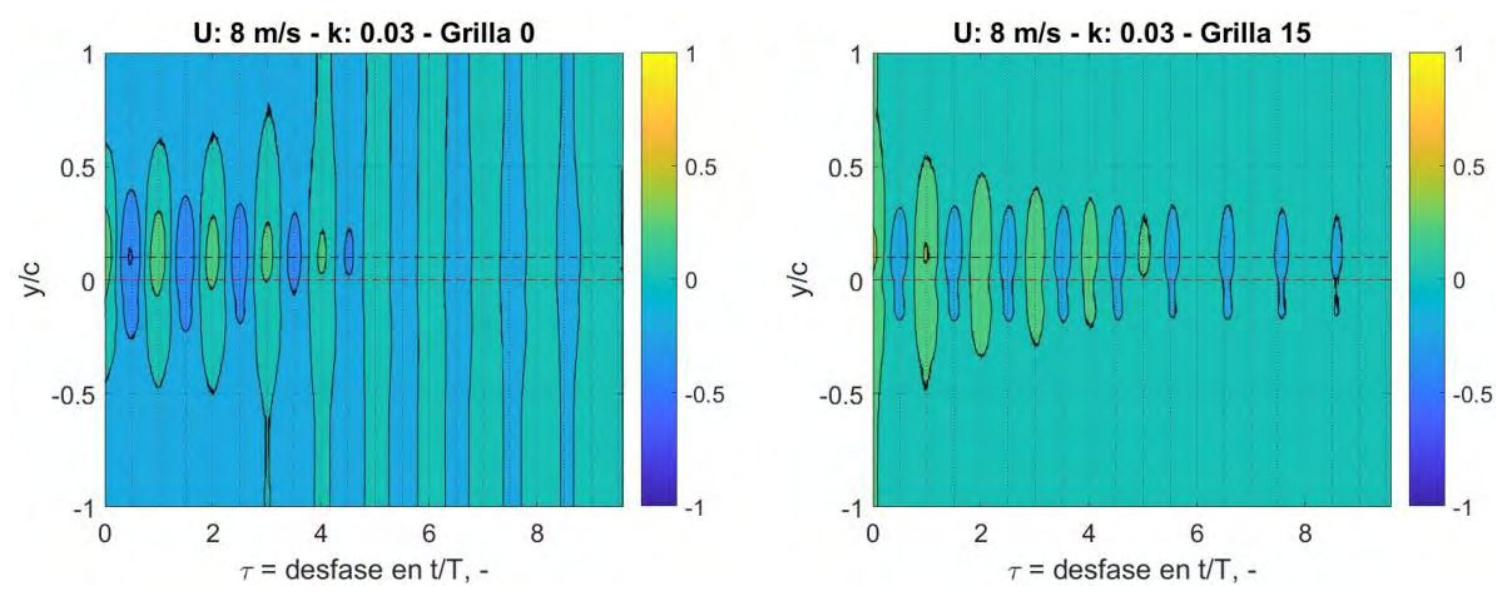

Figura 3.66. Correlación cruzada entre el sensor localizado a la altura del eje de rotación de la estación $0,5 \mathrm{C}$ y los sensores de la estación $1 \mathrm{C}$, para el caso de Reynolds $46.000, \mathrm{k}=0,03$ y ambas intensidades de turbulencia.
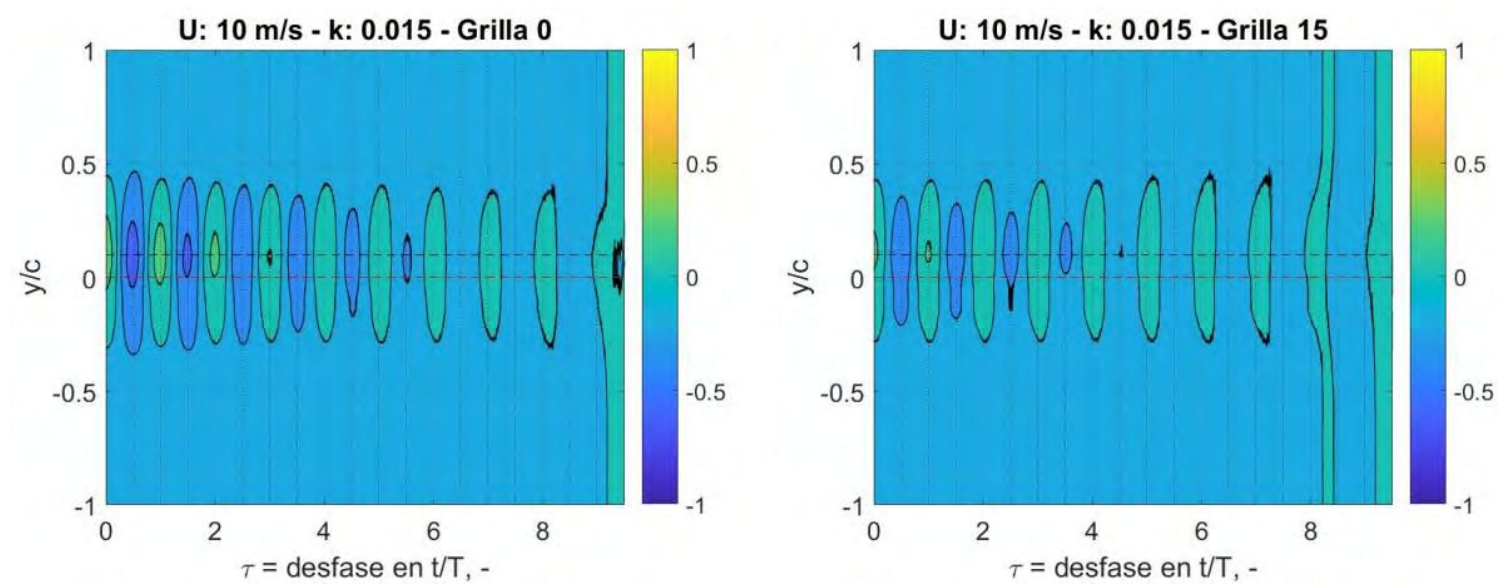

Figura 3.67. Correlación cruzada entre el sensor localizado a la altura del eje de rotación de la estación $0,5 \mathrm{C}$ y los sensores de la estación 1C, para el caso de Reynolds $57.000, k=0,015$ y ambas intensidades de turbulencia.

\subsubsection{Análisis en frecuencias}

Para completar el análisis del campo de presiones en la estela, se procedió a realizar un análisis en el espacio de frecuencias, utilizando las técnicas de transformación de Fourier y Wavelet. El detalle del procedimiento y 


\section{"Estudio aerodinámico experimental en flujo turbulento de bajo Reynolds sobre alas con movimiento de cabeceo"}

significado de estos mecanismos de análisis, para ambas transformaciones, puede ser consultado en el apartado de Transformada de Fourier y Transformada de Wavelet de la sección 2.4 Mediciones de presión en estela.

Dado que ambos análisis están relacionados, por su interés en conocer las componentes en frecuencias de las señales, se mostrarán los resultados en el mismo gráfico. En la sección izquierda se podrá observar el análisis mediante la transformación de wavelet, utilizando como onda de referencia la segunda derivada de la distribución gaussiana, conocida como sombrero mexicano, por su forma. En la derecha, se graficará la densidad de potencia en función de la frecuencia utilizando la transformada rápida de Fourier. En ambas gráficas se agrega como referencia una línea punteada roja con el valor de la frecuencia del movimiento del ala. Cabe destacar, que mientras la transformada de Fourier es exacta en frecuencias, pero no se puede obtener información de la locación temporal en la señal, la transformada Wavelet, es capaz de resolver tanto en frecuencias como en tiempo. Sin embargo, al tener información temporal, pierde exactitud en el campo de las frecuencias la transformada Wavelet, es por eso que en el eje se puede observar la pseudo-frecuencia, que queda determinada en función de la escala de la onda, principalmente.

Desde la Figura 3.69 a la Figura 3.71 se muestran los resultados para el caso de Reynolds 23.000, de ambas intensidades de turbulencia y las tres frecuencias reducidas, para las señales adquiridas media cuerda aguas abajo del borde de fuga con el sensor ubicado a la misma altura que el eje de rotación. Desde la Figura 3.72 hasta la Figura 3.74 se muestran los mismos resultados analizados para la estación ubicada a una cuerda de distancia aguas abajo del borde de fuga. Los resultados para los demás valores de número de Reynolds no se muestran dado que se hallaron resultados muy similares. Desde la Figura 3.75 y Figura 3.76 se puede observar el mapa de Wavelets para la condición de Reynolds 23.000 en la máxima frecuencia reducida y ambas turbulencias, pero comparando las señales de distintos sensores, ubicados hacia arriba y abajo del eje de rotación.

Como primer resultado se puede observar como el pico de frecuencias hallado en la densidad de potencia coincida perfectamente con la frecuencia del movimiento del ala, como era esperable, para todos los casos analizados. Esto nos indica que los desprendimientos vorticosos encontrados en la estela, están dominados por la cinemática del ala. A su vez, como se viene demostrando a lo largo de los análisis anteriores, al aumentar la intensidad de turbulencia, la densidad de potencia disminuye, indicando que la energía se encuentra disipada, producto del intercambio de energía entre las capas de flujo externo. Otro punto por denotar, observando los resultados del mapa de Wavelets, es que para la estación ubicada media cuerda aguas abajo, el primer vórtice ocurre en el valor del 70\% del ciclo, es decir 0,2 periodos después que el ala alcanzó el máximo valor de ángulo de ataque. Luego de ellos, los vórtices aparecen espaciados cada 0,5 periodos, lo que indica el fin de cada rampa (ascendente o descendente), como se puede observar en la Figura 3.68.

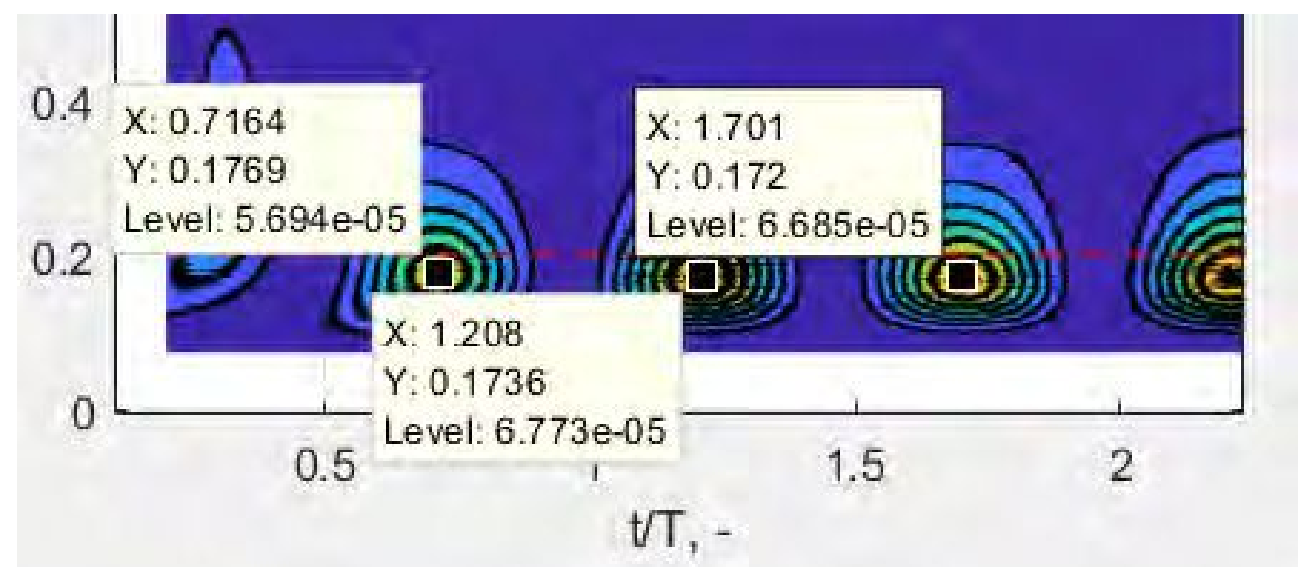

Figura 3.68. Análisis de la aparición temporal de los vórtices para la condición de Reynolds 23.000 con mínima turbulencia en la estación 0,5C y k=0,015. 
$\mathrm{Al}$ analizar las mediciones realizadas una cuerda aguas abajo del borde de fuga del perfil (Figura 3.72 a Figura 3.74), podemos observar en el espectro, como la energía en el flujo decreció un orden de magnitud, con lo cual, en el mapa de Wavelet, se utilizó otra escala para poder dibujar los contornos y poder visualizar las zonas periódicas. Este fenómeno era de esperarse dado que al estar más alejada la estación de medición, la energía de estos vórtices decrece, y empiezan a generar una estela turbulenta sin tantas estructuras coherentes.

Otro punto para destacar es que, al aumentar la frecuencia reducida del movimiento, la banda de frecuencias en la señal de presiones empieza a expandirse, demostrando que en el fluido hay vórtices con mayor variedad de frecuencias, aunque siempre concentradas en torno a la frecuencia del movimiento. Este efecto se ve destacado en el mapa de Wavelets, donde las zonas coloreadas se ven más "estiradas". El mismo efecto se observa en la densidad de potencia, donde el pico deja de ser tan estrecho.

\subsubsection{Modelo con perfil SD8020}

A continuación, se muestran los resultados de las correlaciones cruzadas y mapas de wavelets, junto con los espectros de densidad de potencia, para los casos dinámicos, en ambas condiciones de turbulencia para el modelo alar con perfil aerodinámico SD8020.

\subsubsection{Correlaciones Cruzadas en la estación 0,5C}

Las correlaciones cruzadas, para esta sección, se realizaron dejando el rack de presiones fijo en una estación localizada media cuerda aguas abajo del borde de fuga, y realizando la correlación cruzada entre la toma ubicada a la altura del eje de rotación, respecto al resto de las tomas. De esta forma, podemos analizar qué tan parecidas son las señales, y, por ende, como sabemos que la toma de referencia está inmersa dentro de la estela, conocer el tamaño de la misma. Estos resultados se encuentran en la Figura 3.77. Como referencia se localizan líneas punteadas a la altura del eje de rotación y un cuarto de cuerda hacia arriba y hacia abajo. En la columna izquierda se muestran los resultados para el caso de mínima intensidad de turbulencia, y en la derecha para turbulencia del $1 \%$. Solo se muestran los casos para Reynolds 23.000 dado que se hallaron resultados similares en las otras condiciones de número de Reynolds. Los resultados son los correspondientes a la estación localizada media cuerda aguas abajo del borde de fuga, producto de que una cuerda aguas abajo, la correlación es muy baja y no se encuentran definidos los vórtices desprendidos por el ala.

Para las distintas frecuencias reducidas analizadas, y distintos niveles de turbulencia, el centro de la estela se encuentra desplazado hacia arriba en un $10 \%$ de la cuerda, aproximadamente. Considerando dicho corrimiento, el tamaño de la estela, nuevamente para todos los casos, tiene un tamaño aproximado de media cuerda, cuarto de cuerda hacia arriba y cuarto de cuerda hacia abajo. El espaciado temporal de los picos de correlación corresponde con la frecuencia del movimiento, por lo que los vórtices desprendidos por el ala están en sincronismo con el movimiento realizado, como era de esperarse. Al compararse los resultados entre los dos niveles de turbulencia ensayados, no se presentan modificaciones en los valores de correlación, como si fueron hallados en el modelo de placa plana. 

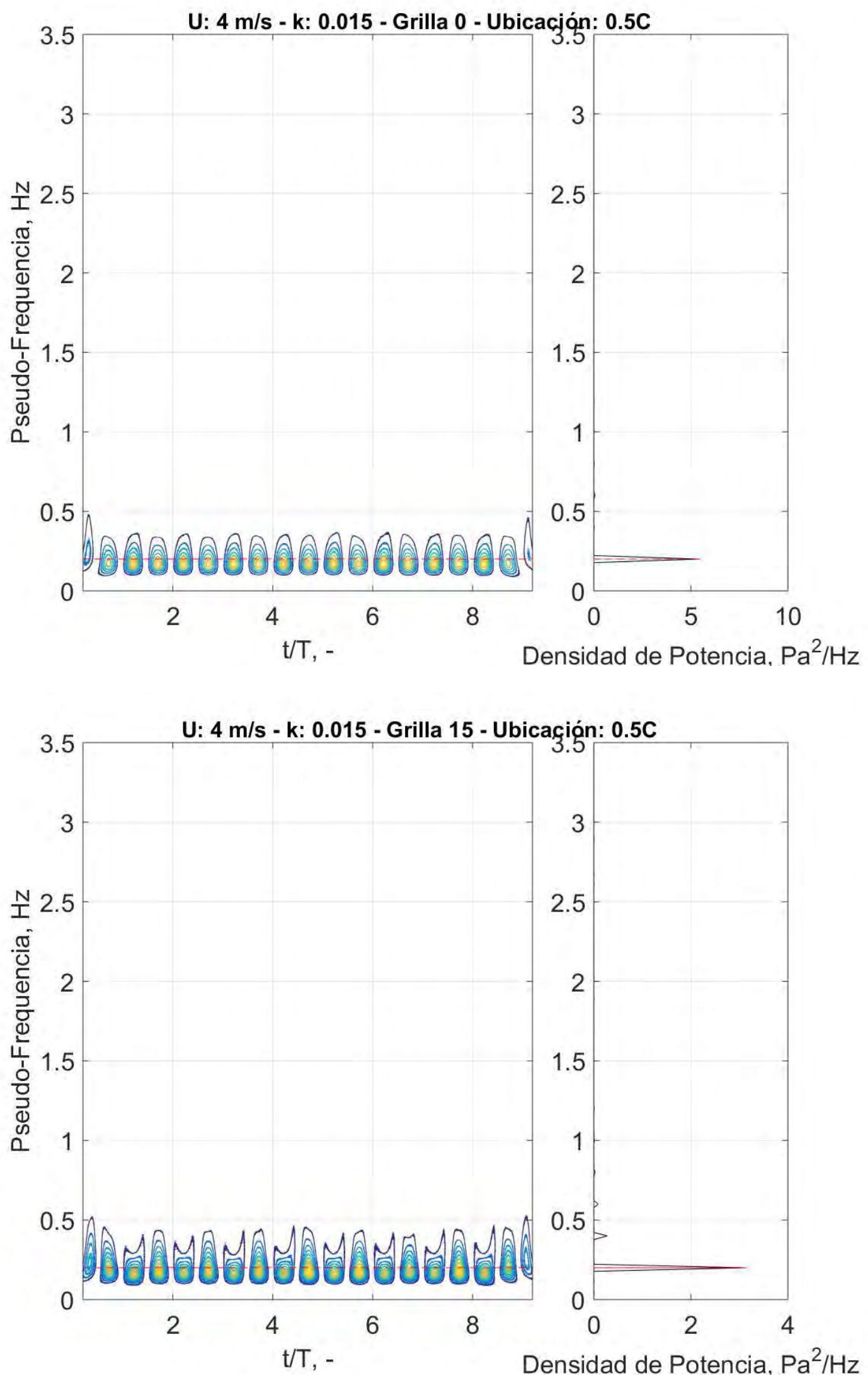

Figura 3.69. Mapa de Wavelets y Densidad de potencia. Reynolds $23.000, \mathbf{k}=\mathbf{0 , 0 1 5}$ para ubicación media cuerda aguas abajo del borde de fuga y ambas intensidades de turbulencia. 

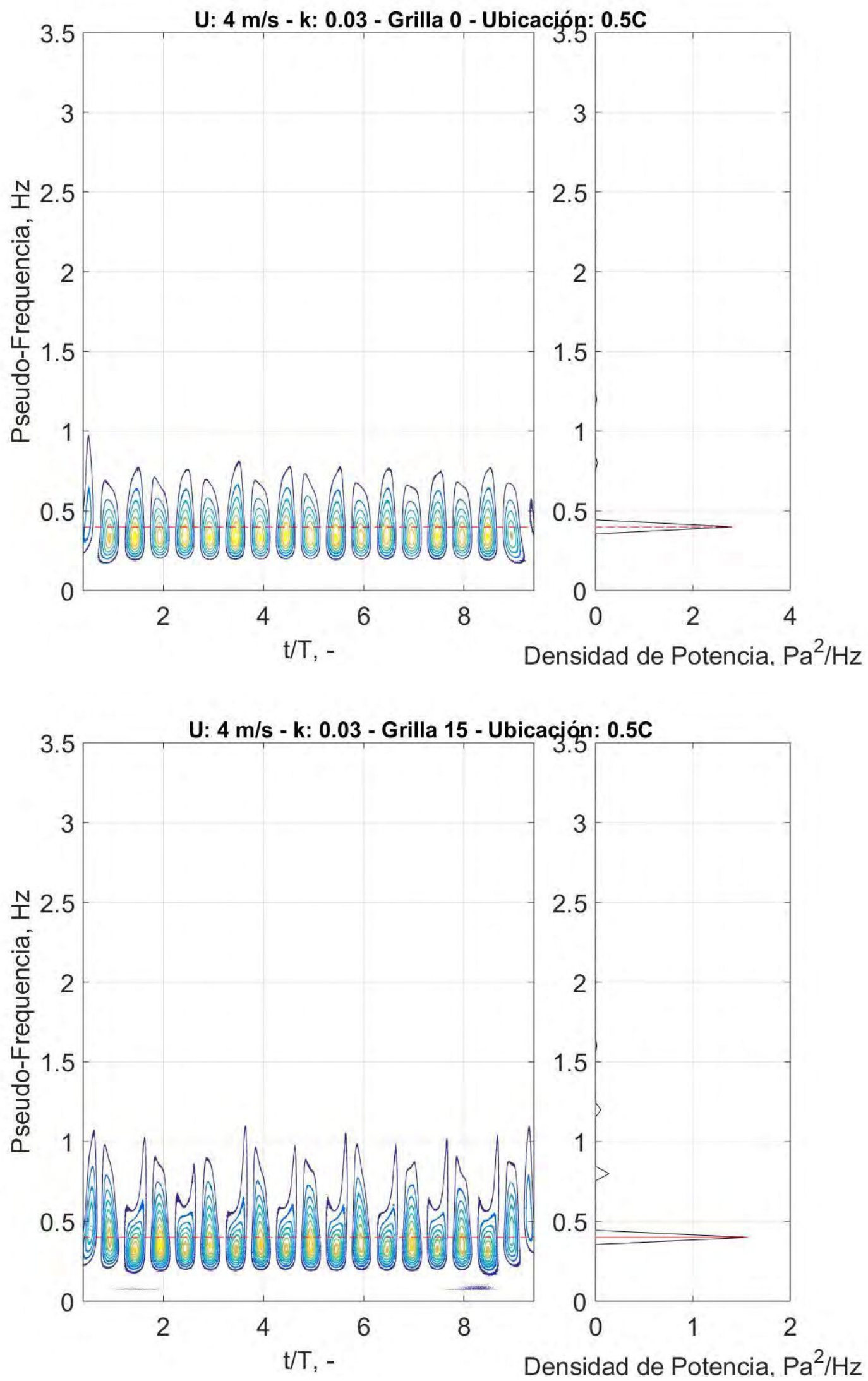

Figura 3.70. Mapa de Wavelets y Densidad de potencia. Reynolds $23.000, k=0,03$ para ubicación media cuerda aguas abajo del borde de fuga y ambas intensidades de turbulencia. 

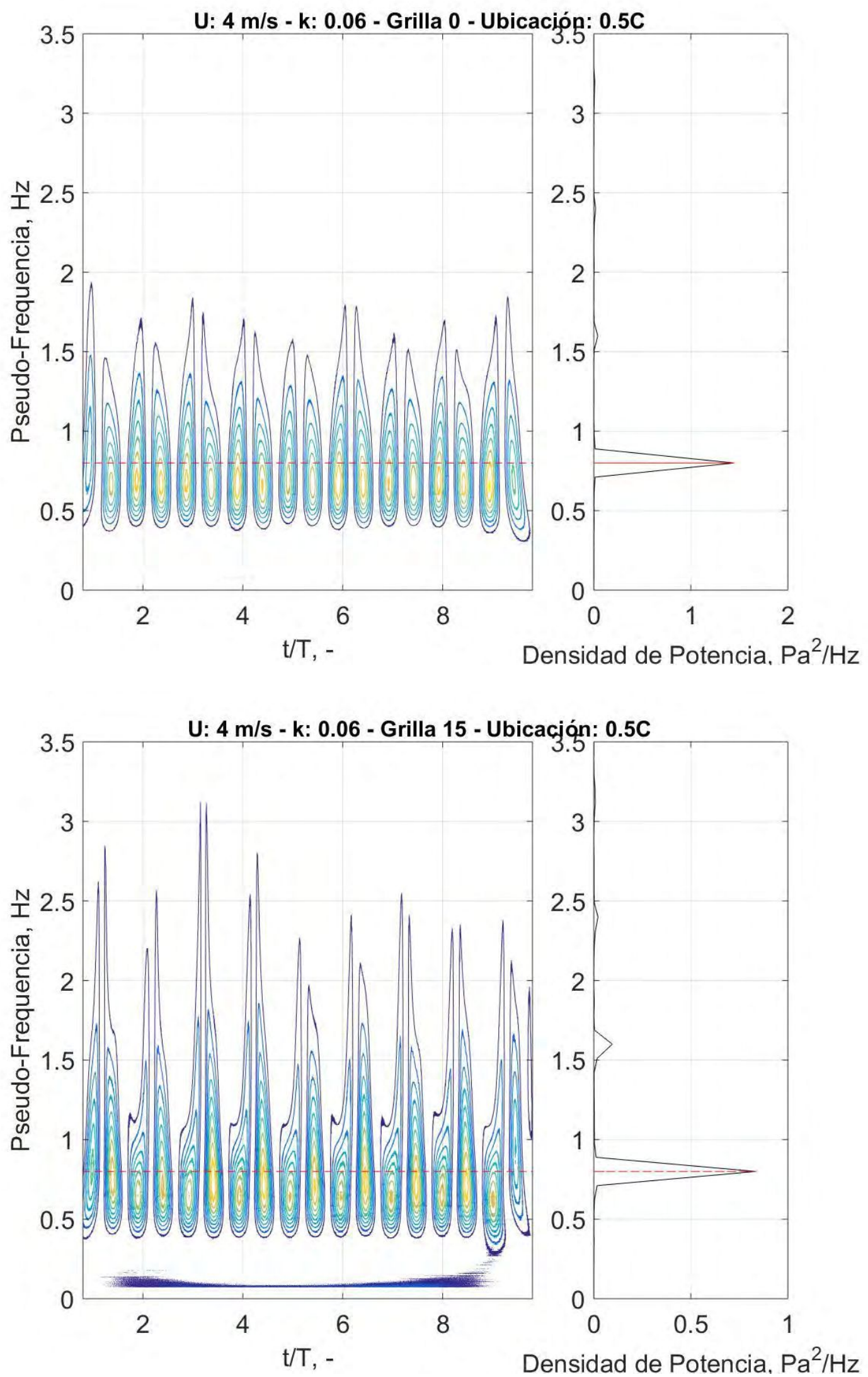

Figura 3.71. Mapa de Wavelets y Densidad de potencia. Reynolds $23.000, k=0,06$ para ubicación media cuerda aguas abajo del borde de fuga y ambas intensidades de turbulencia. 

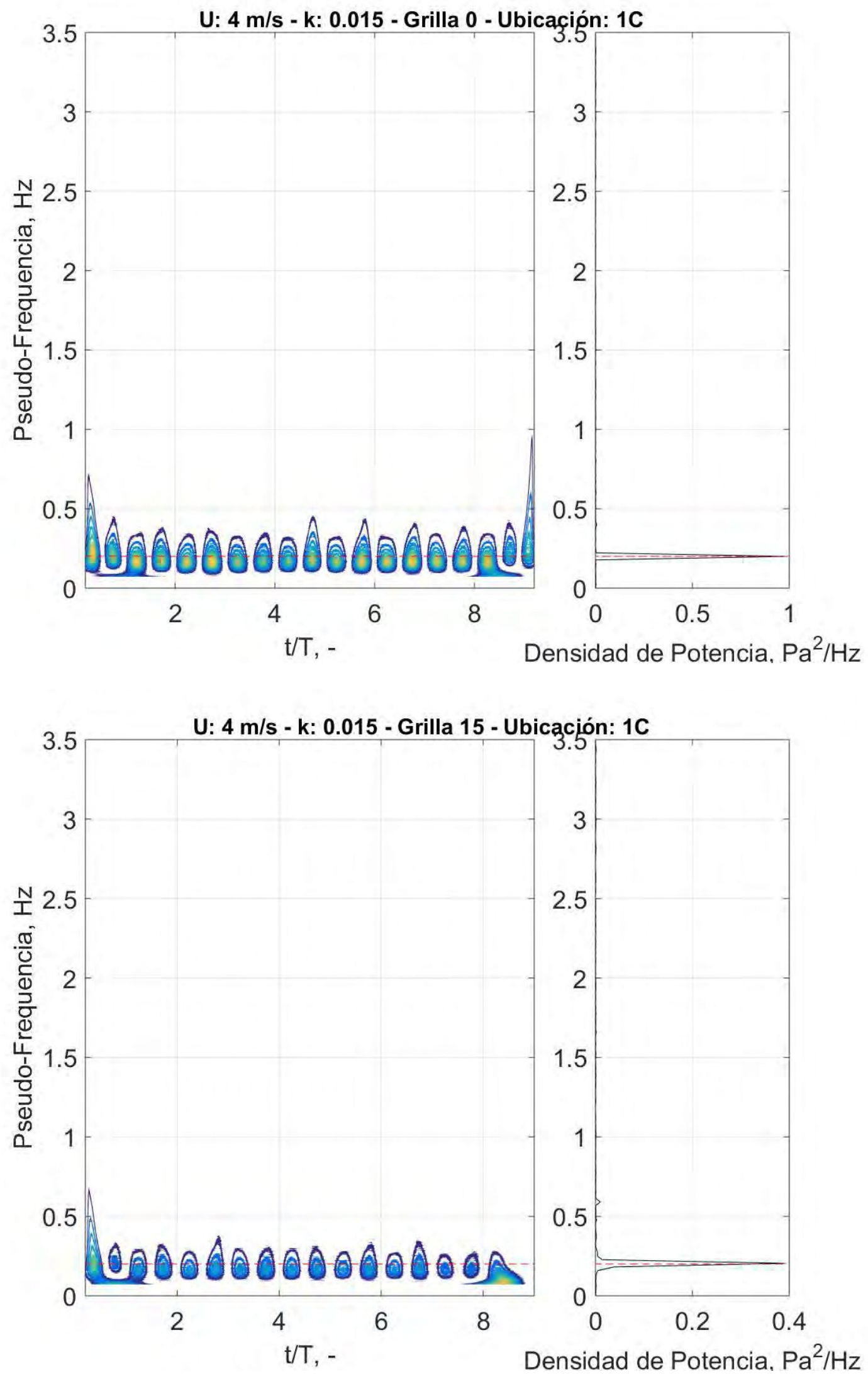

Figura 3.72. Mapa de Wavelets y Densidad de potencia. Reynolds 23.000, $\mathrm{k}=\mathbf{0 , 0 1 5}$ para ubicación una cuerda aguas abajo del borde de fuga y ambas intensidades de turbulencia. 

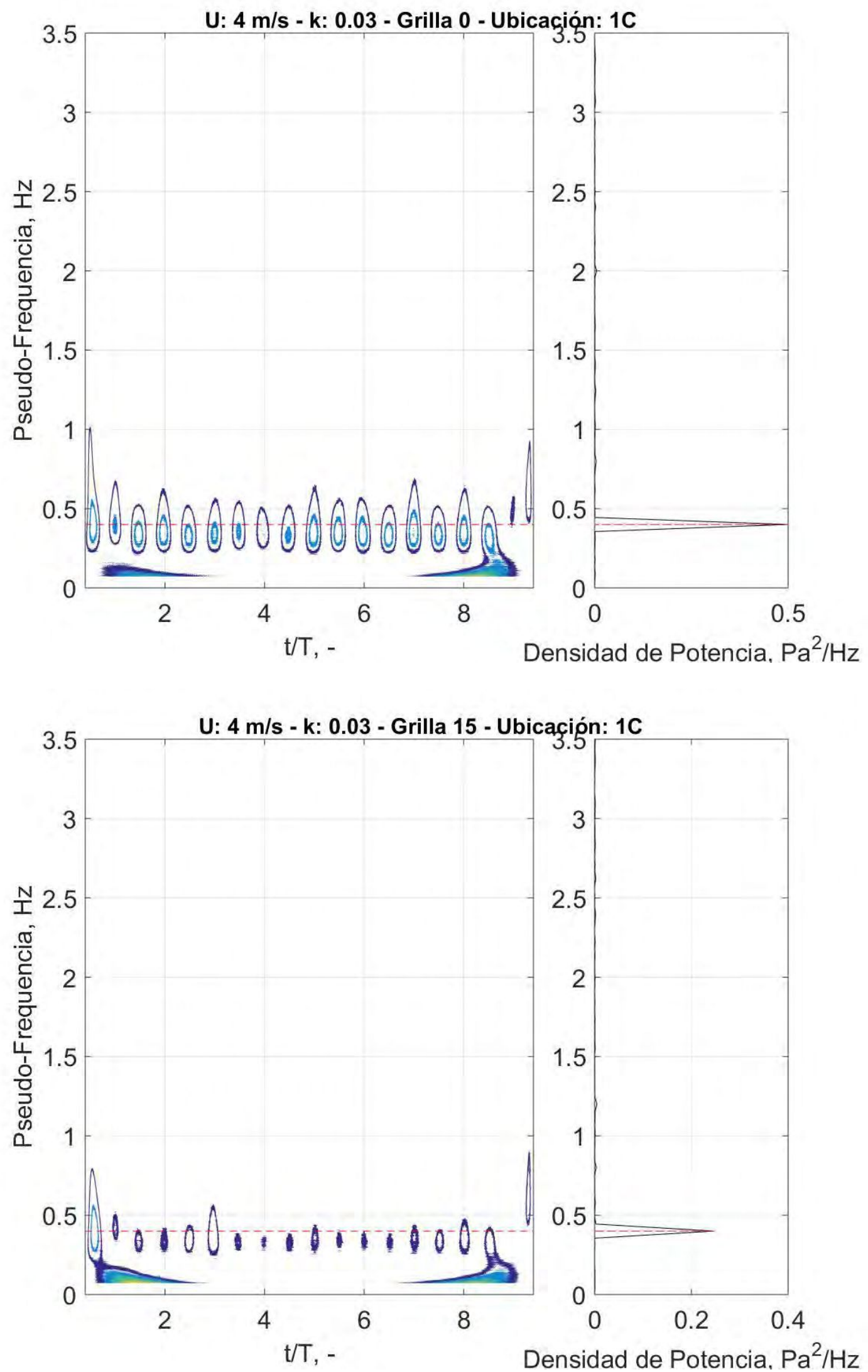

Figura 3.73. Mapa de Wavelets y Densidad de potencia. Reynolds $23.000, \mathrm{k}=\mathbf{0 , 0 3}$ para ubicación una cuerda aguas abajo del borde de fuga $y$ ambas intensidades de turbulencia. 

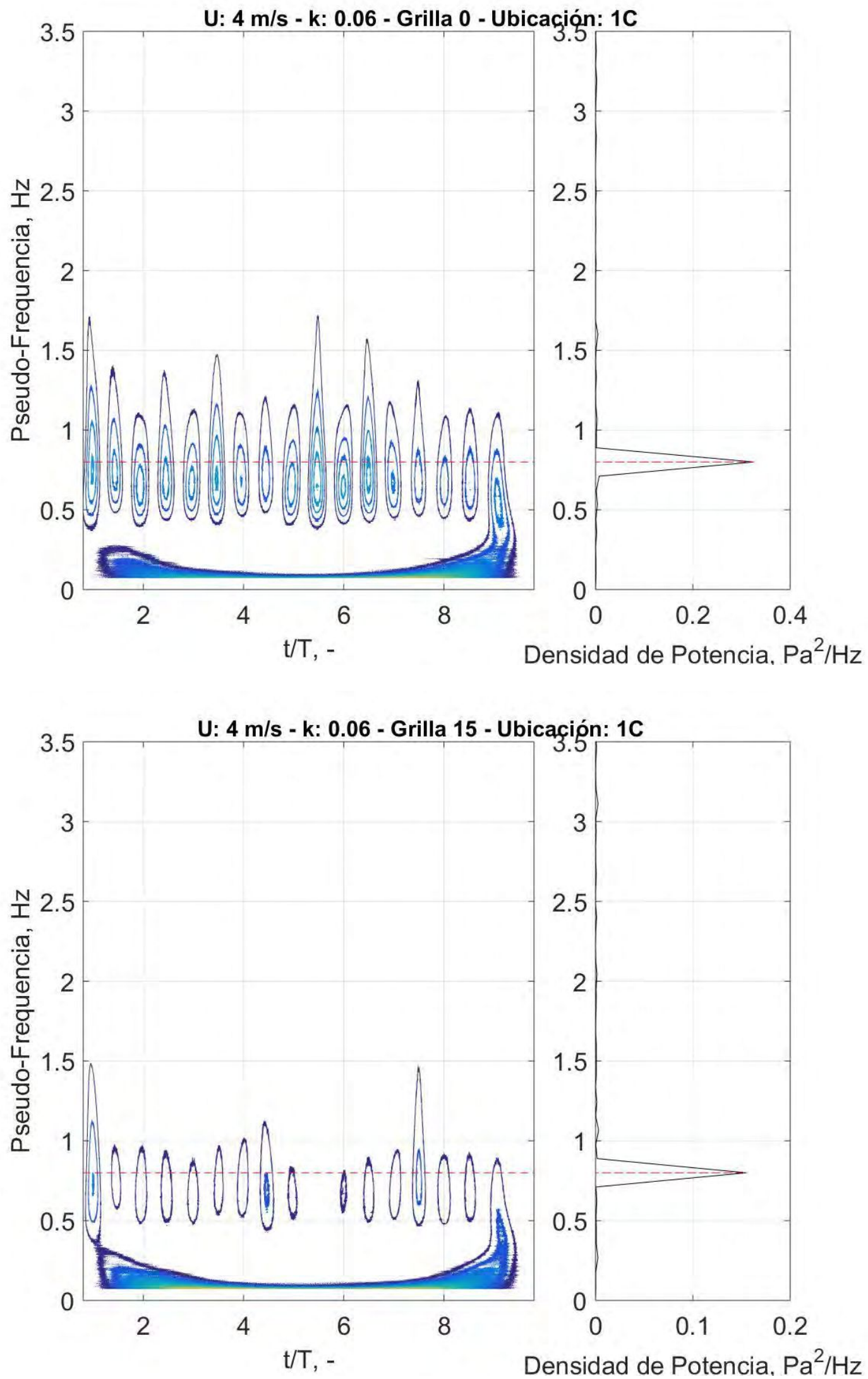

Figura 3.74. Mapa de Wavelets y Densidad de potencia. Reynolds 23.000, $\mathrm{k}=\mathbf{0 , 0 6}$ para ubicación una cuerda aguas abajo del borde de fuga y ambas intensidades de turbulencia. 


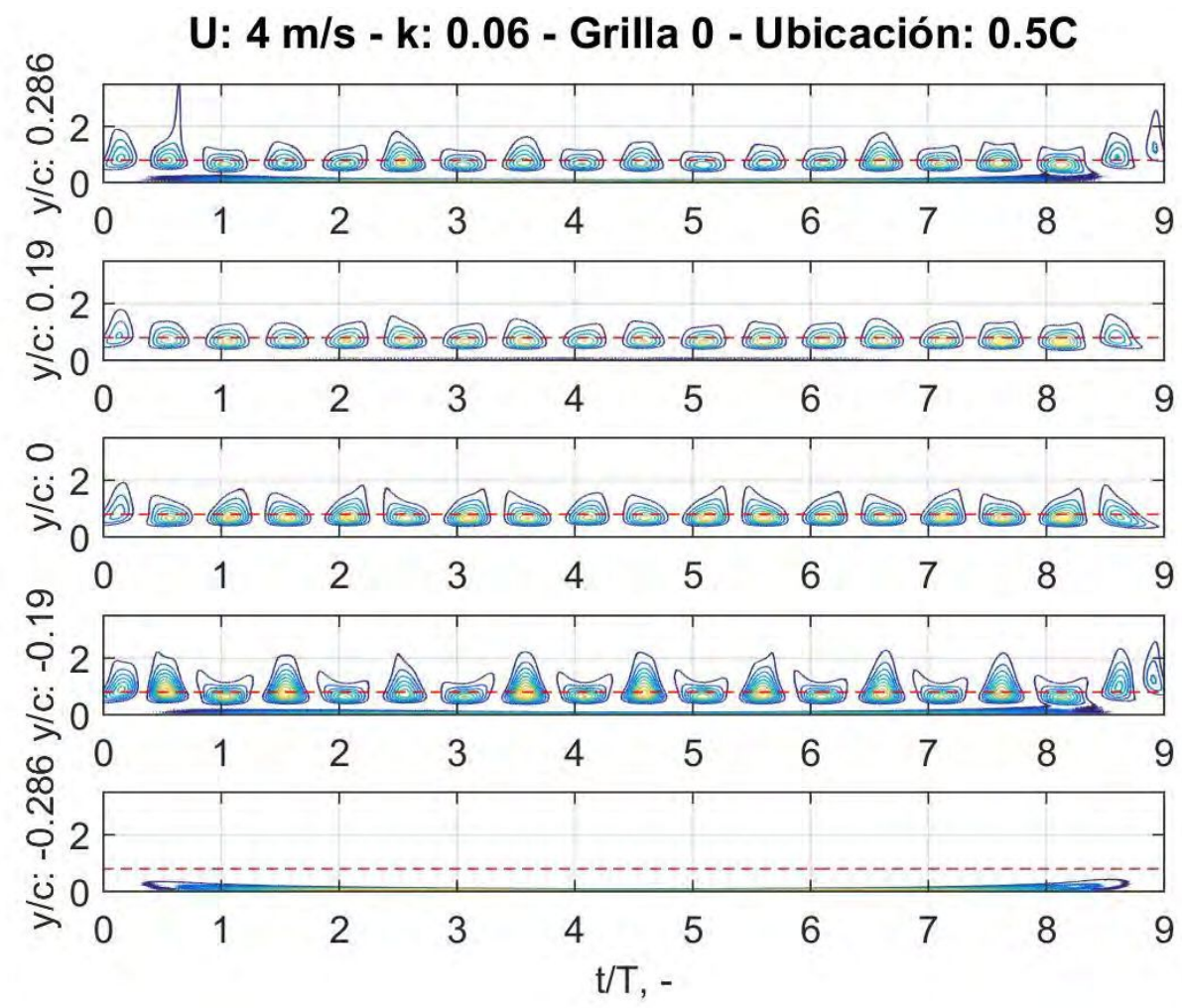

Figura 3.75. Mapa de Wavelets de distintos sensores, para el caso de Reynolds 23.000, k=0,06, mínima turbulencia y el rack alejado media cuerda de distancia.

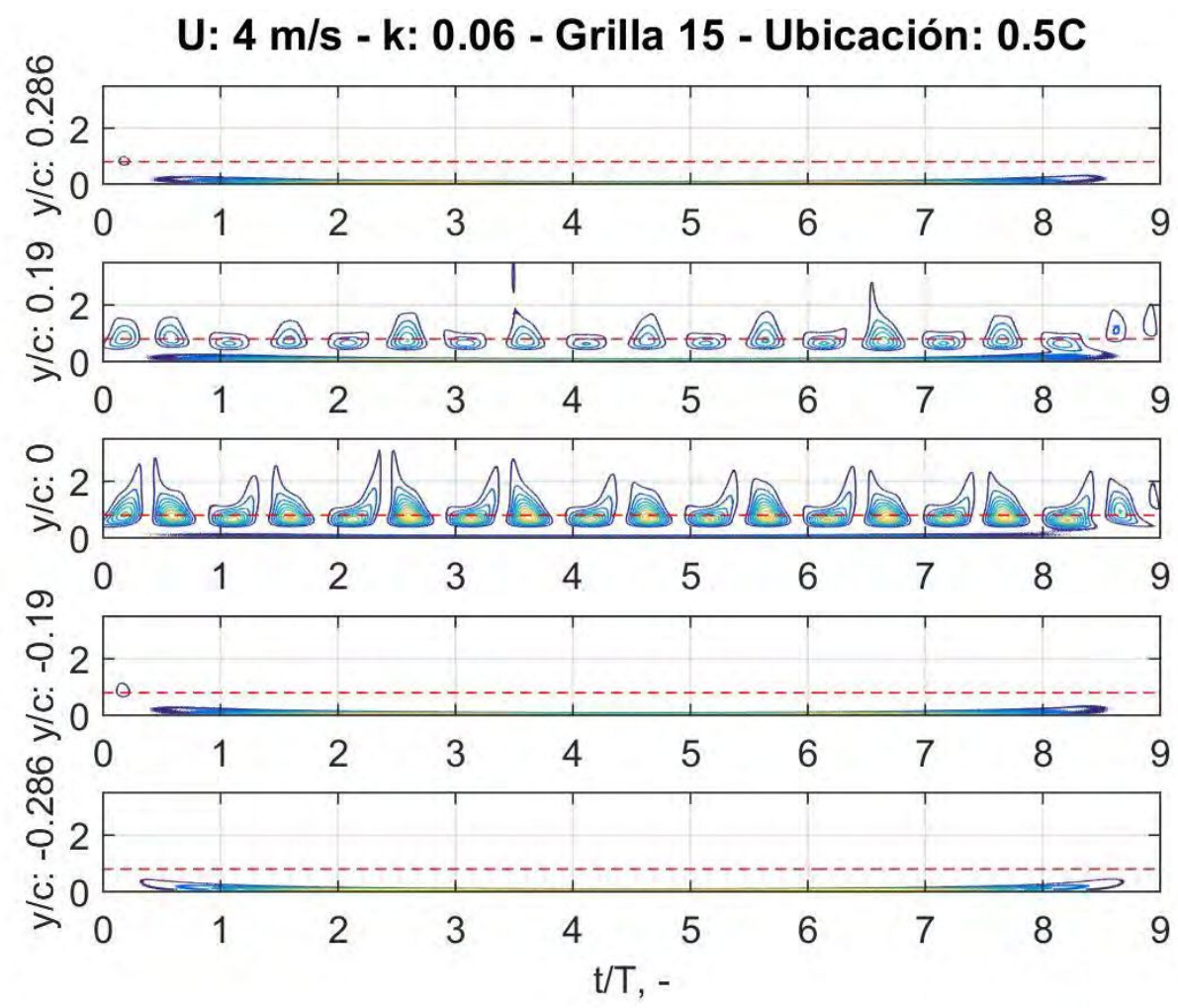

Figura 3.76. Mapa de Wavelets de distintos sensores, para el caso de Reynolds 23.000, $\mathrm{k}=0,06$, máxima intensidad de turbulencia y el rack alejado media cuerda de distancia. 

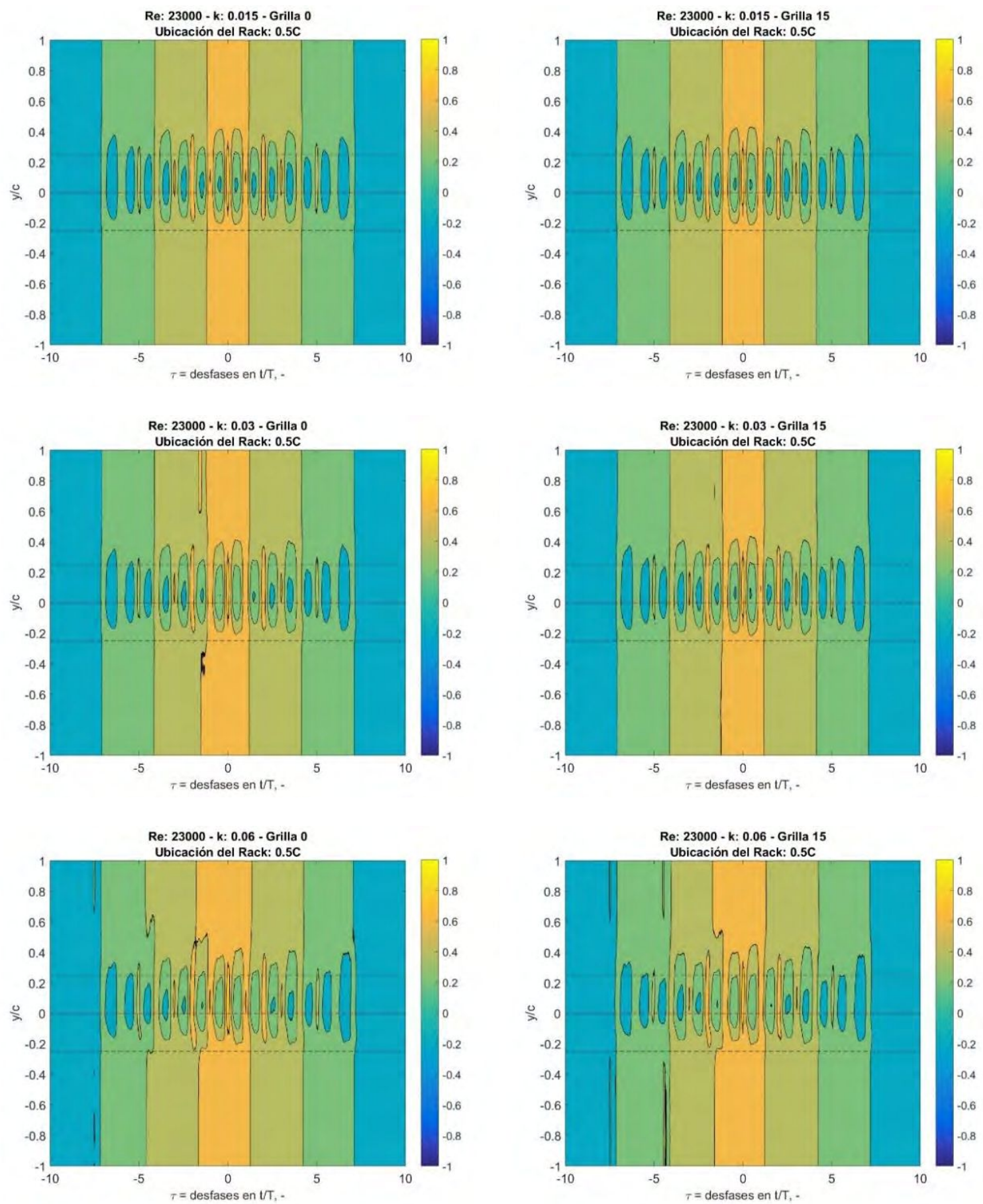

Figura 3.77. Correlación cruzada entre tomas de una estación, respecto al sensor localizado en el eje de rotación para distintas frecuencias reducidas e intensidad de turbulencia. Re: 23000 , estación a media cuerda aguas abajo.

\subsubsection{Correlaciones Cruzadas en la estación 1C}

En esta sub-sección se muestran el mismo análisis realizado previamente, pero ahora para la estación localizada una cuerda aguas abajo del borde de fuga del modelo alar. Cómo fue mencionado previamente, para esta estación, producto de la lejanía del perfil, y de su perfil aerodinámica, los vórtices que pudimos observar para la placa plana ya no están presentes. Nuevamente, la columna izquierda representa los resultados a mínima intensidad de turbulencia, mientras que la columna derecha con la intensidad mayor. Los resultados son los mostrados en la Figura 3.78. 

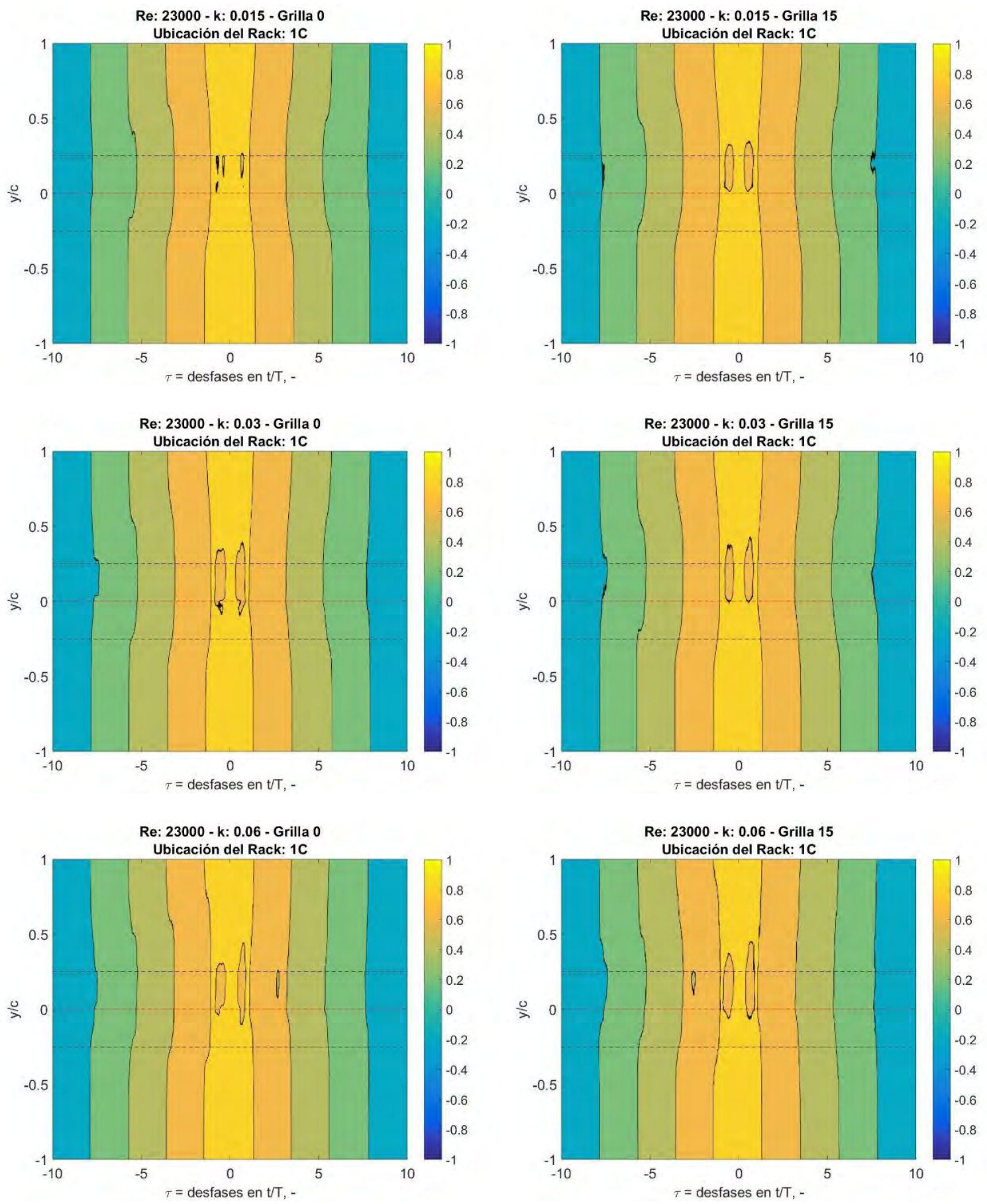

Figura 3.78. Correlación cruzada entre tomas de una estación, respecto al sensor localizado en el eje de rotación para distintas frecuencias reducidas e intensidad de turbulencia. Re: 23000 , estación a una cuerda aguas abajo.

\subsubsection{Correlación cruzada entre estación 0,5C y $1 C$}

Siguiendo con el mismo formato de gráficos, de dos columnas, con la mínima intensidad de turbulencia en la columna izquierda, y la máxima en la derecha, en la Figura 3.79 se presentan los resultados de la correlación cruzada entre el sensor localizado a la altura del eje de rotación media cuerda aguas abajo, respecto a todos los sensores en la estación una cuerda aguas abajo. En los gráficos se puede observar como para todos los casos el centro de los vórtices, denotado por el valor de máxima correlación, se ha desplazado hacia arriba, respecto al valor inicial de 0,1 cuerdas encontrado en la estación más cercana al borde de fuga, al trasladarse aguas abajo media cuerda. A su vez, la periodicidad sigue marcada por la frecuencia del movimiento del ala, sin encontrarse 
un desfasaje en dicho sentido. Nuevamente, para este perfil, no se observan diferencias en los valores de correlación cuando se modifica la intensidad de turbulencia.
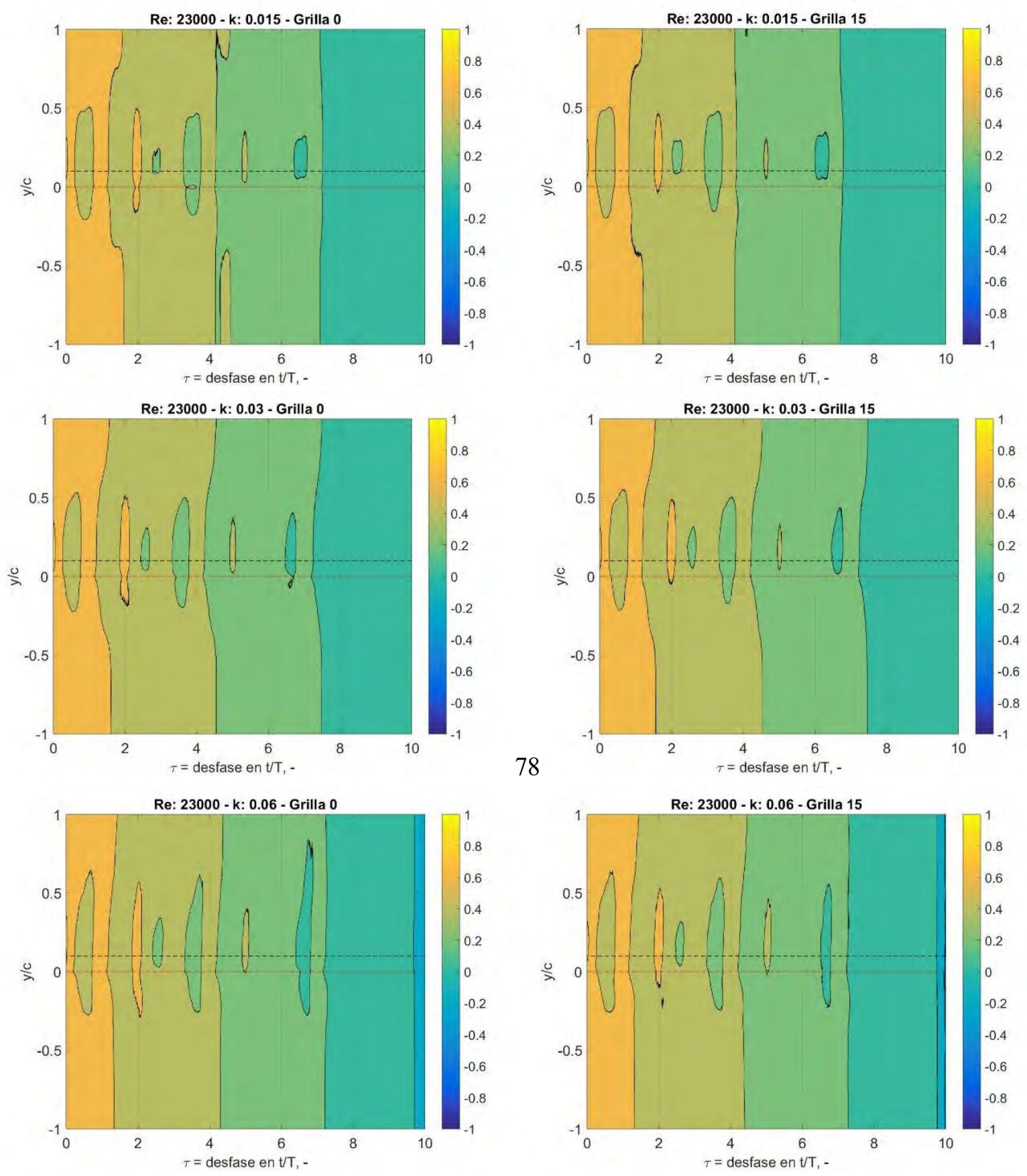

Figura 3.79. Correlación cruzada entre la toma ubicada en el eje de rotación de la estación 0,5C con todas las tomas de presión de la estación 1C. Reynolds 23000 y distintas frecuencias reducidas e intensidades de turbulencia.

\subsubsection{Análisis en frecuencias}

Se realizó un mapa de wavelets utilizando la segunda derivada de la función gaussiana (sombrero mexicano), para localizar los máximos y mínimos, al igual que como fue realizado con el perfil de placa plana. A continuación, desde la Figura 3.80 hasta la Figura 3.85, se muestran los resultados encontrados denotando con una línea el valor de la frecuencia del movimiento, para analizar, tanto en la frecuencia de los espectros de 


\section{"Estudio aerodinámico experimental en flujo turbulento de bajo Reynolds sobre alas con movimiento de cabeceo"}

densidad de potencia, como en el mapa de wavelets, la frecuencia de las presiones en comparación con la del movimiento.

$\mathrm{Al}$ analizar los resultados de las Figura 3.80 a Figura 3.82, salvo para la condición de máxima frecuencia reducida, no se observan mayores diferencias entre los mapas de Wavelets, o en los espectros. Al analizar este caso, se puede observar como los máximos y mínimos es encuentran más concentrados para la mayor intensidad de turbulencia (grilla 15), sin tanta dispersión en el espectro de frecuencias. Este fenómeno no se puede apreciar en los espectros de densidad de potencia, utilizando el método de Fourier.

Cuando se incrementa el número de Reynolds, Figura 3.83 a Figura 3.85, los resultados hallados son muy similares, encontrando en el espectro de potencia un gran pico correspondiente con la frecuencia del movimiento, pero también apareciendo un segundo pico de menor intensidad. Este segundo pico, al comparar los resultados con el mapa de wavelets, se puede observar que se condicen con una deformación en los círculos, donde aparecen estas distorsiones en frecuencias mayores.

\subsection{Visualizaciones}

En esta sección, se mostrarán los resultados hallados al implementar la técnica de visualización de inyección de humo en la corriente libre, e iluminación de un plano mediante un láser, de color verde. Las filmaciones fueron realizadas con una cámara semi-profesional Nikon Coolpix L830, desde una vista lateral, aunque no perpendicular al plano iluminado. A partir de las filmaciones y fotografías se obtuvieron las capturas de los cuadros para el perfil SD8020, tanto en condiciones estáticas, como en dinámicas a distintas frecuencias. Es importante mencionar que las visualizaciones fueron realizadas únicamente para la condición de mínima turbulencia, es decir, sin la grilla generadora de turbulencia. No se realizaron las visualizaciones con mayor intensidad de turbulencia, dado que las diferencias esperadas a encontrar en el patrón de flujo sobre el perfil no pueden ser representadas por el método de visualización implementado Para poder apreciar las diferencias, deberíamos poder realizar visualizaciones sobre la capa límite del perfil, pero esto no es posible en las instalaciones utilizadas.

\subsubsection{Condiciones estáticas en perfil SD8020}

En la Figura 3.86 se pueden ver capturas de las filmaciones realizadas para condiciones de ángulo estático. Los ángulos elegidos fueron los que se consideraron más relevantes en función de los resultados obtenidos en la curva de sustentación en función del ángulo de ataque. Cómo se puede observar, para el ángulo de ataque nulo se encuentra la presencia de una separación de flujo en el borde de fuga, la cual desaparece al incrementar el ángulo de ataque hasta $5^{\circ}$. Esta diferencia puede ser la generadora de una no linealidad en la curva de sustentación en las inmediaciones del ángulo de ataque nulo. Luego al incrementar el ángulo de ataque se puede observar como hasta $10^{\circ}$ el flujo se mantiene adherido a la superficie alar, mientras que para un ángulo de $12^{\circ}$ se observa un desprendimiento masivo del flujo desde el borde de ataque (demarcado con línea naranja), lo que genera una pérdida en la sustentación, así como un incremento notorio de la resistencia. Al llegar hasta los $15^{\circ}$ se pueden observar desprendimientos vorticosos, desde el borde de fuga que, en función de los videos grabados en dicha condición, a priori, son aleatorios, y no siguen un patrón periódico. Estos desprendimientos se encuentran denotados con una línea naranja. 

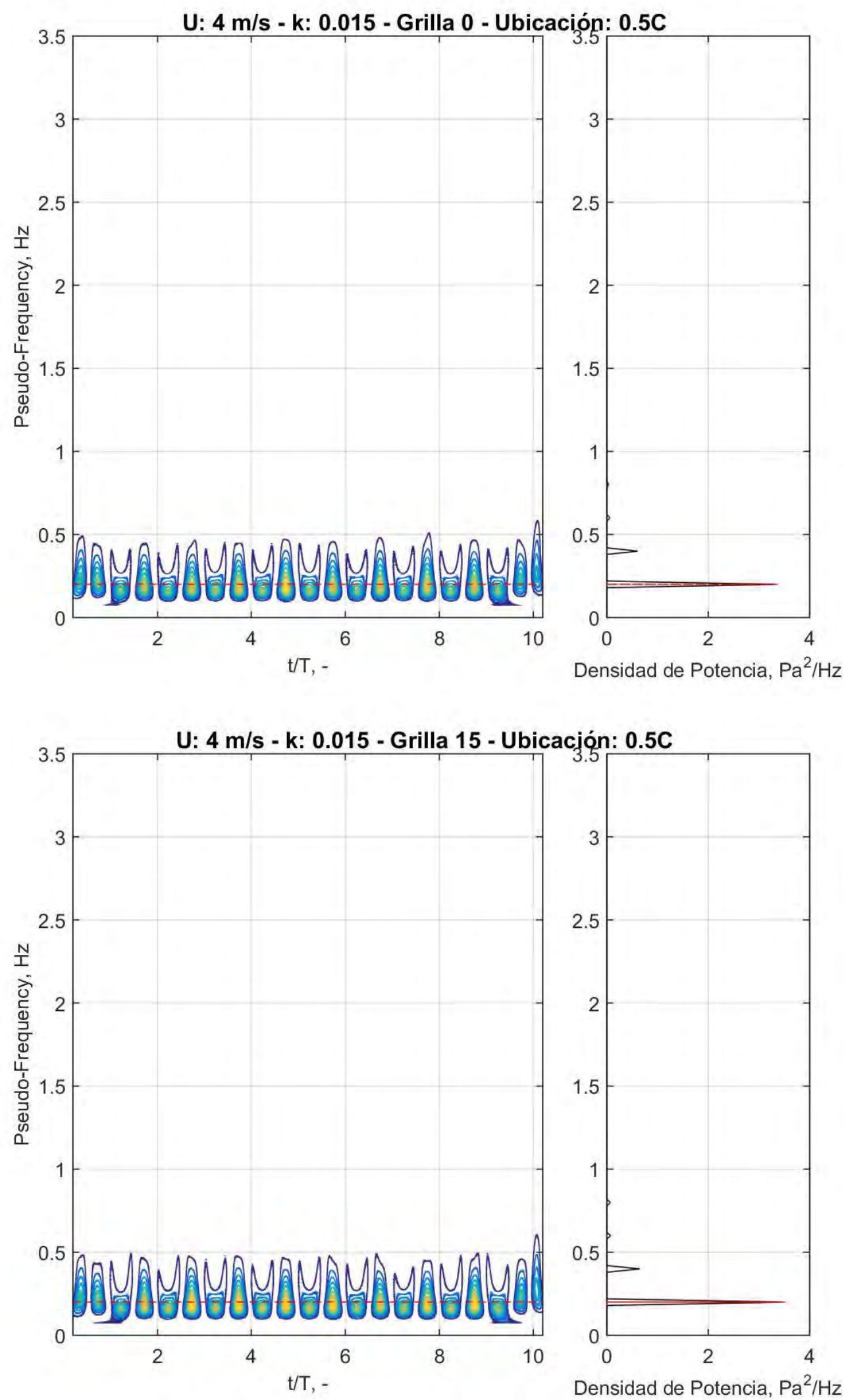

Figura 3.80. Mapa de Wavelets y Densidad de potencia. Reynolds 23.000, $\mathrm{k}=\mathbf{0 , 0 1 5}$ para ubicación media cuerda aguas abajo del borde de fuga y ambas intensidades de turbulencia. Modelo Alar: Perfil SD8020 

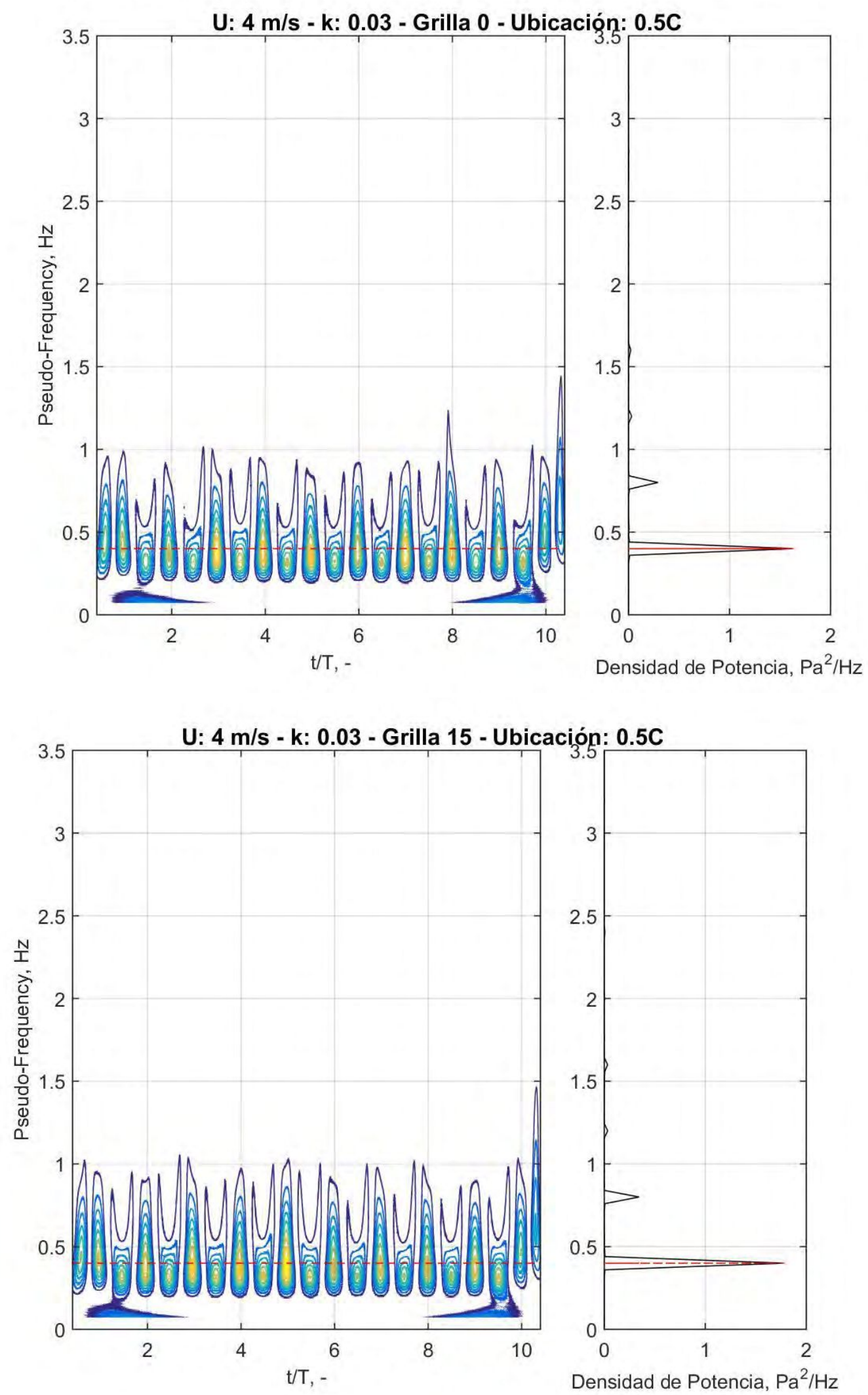

Figura 3.81. Mapa de Wavelets y Densidad de potencia. Reynolds 23.000, $\mathrm{k}=\mathbf{0 , 0 3}$ para ubicación media cuerda aguas abajo del borde de fuga y ambas intensidades de turbulencia. Modelo Alar: Perfil SD8020 

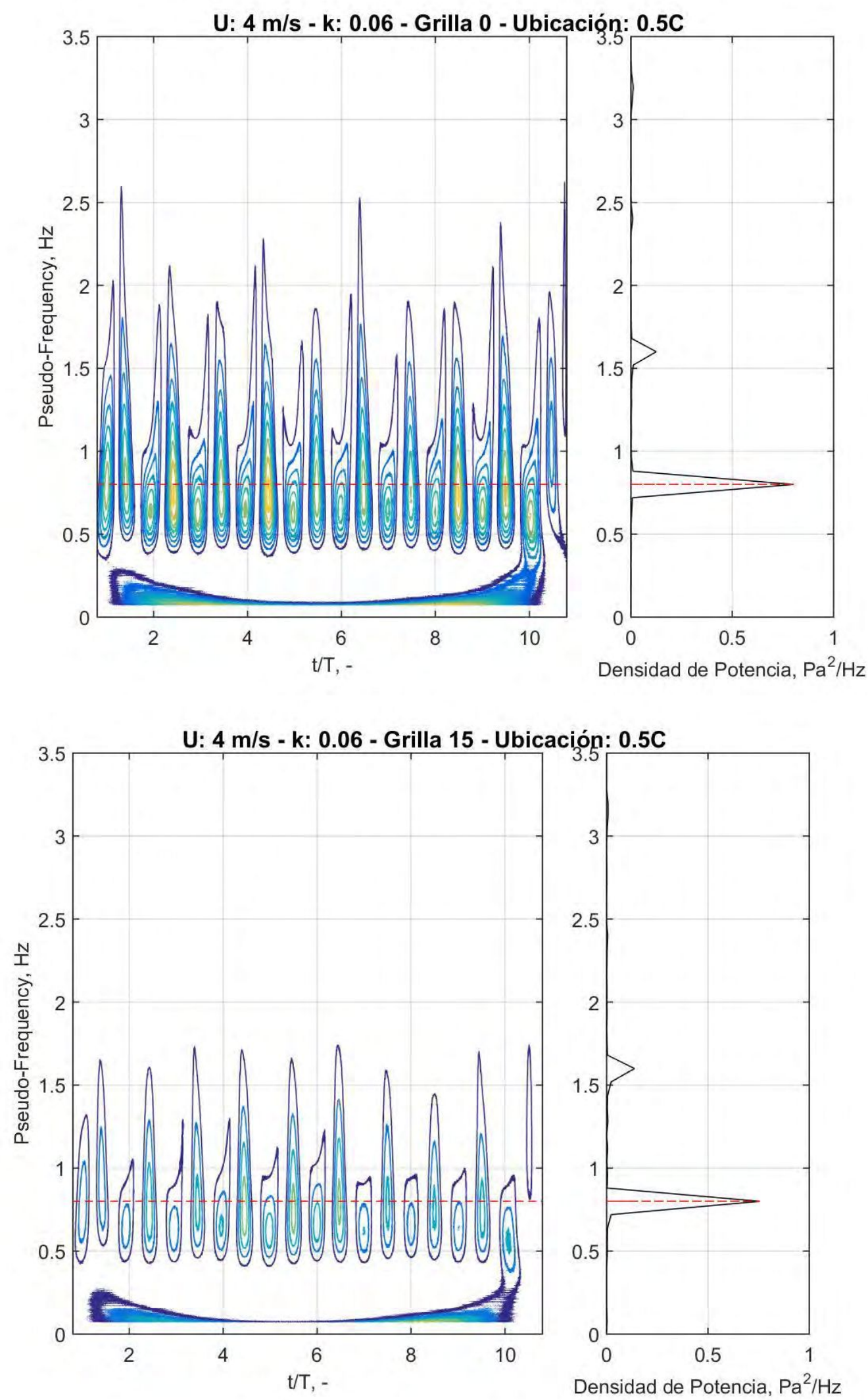

Figura 3.82. Mapa de Wavelets y Densidad de potencia. Reynolds 23.000, $\mathrm{k}=\mathbf{0 , 0 6}$ para ubicación media cuerda aguas abajo del borde de fuga y ambas intensidades de turbulencia. Modelo Alar: Perfil SD8020 
U: 8 m/s - k: 0.015 - Grilla 0 - Ubicación: 0.5C
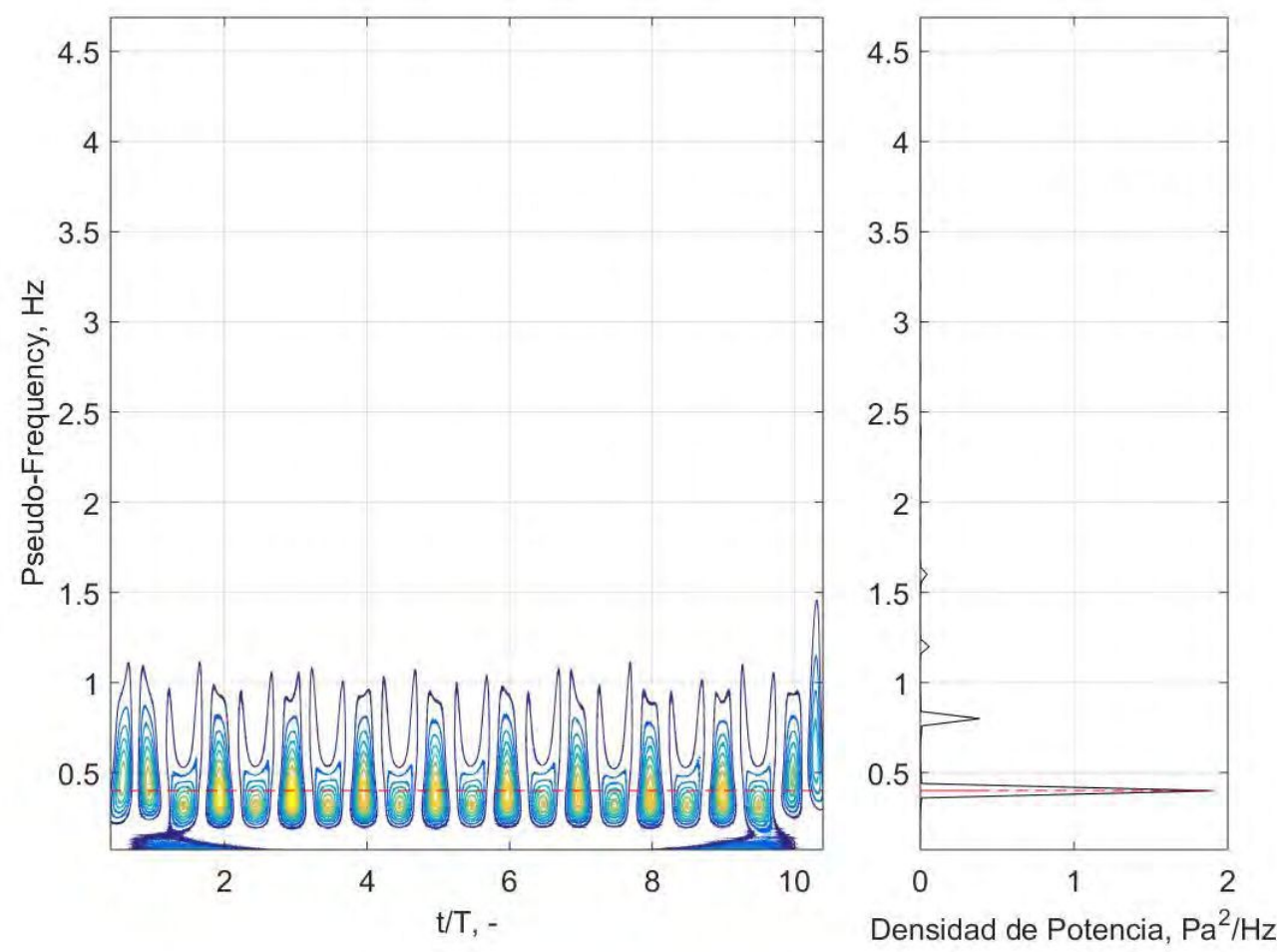

U: 8 m/s - k: 0.015 - Grilla 15 - Ubicación: 0.5C
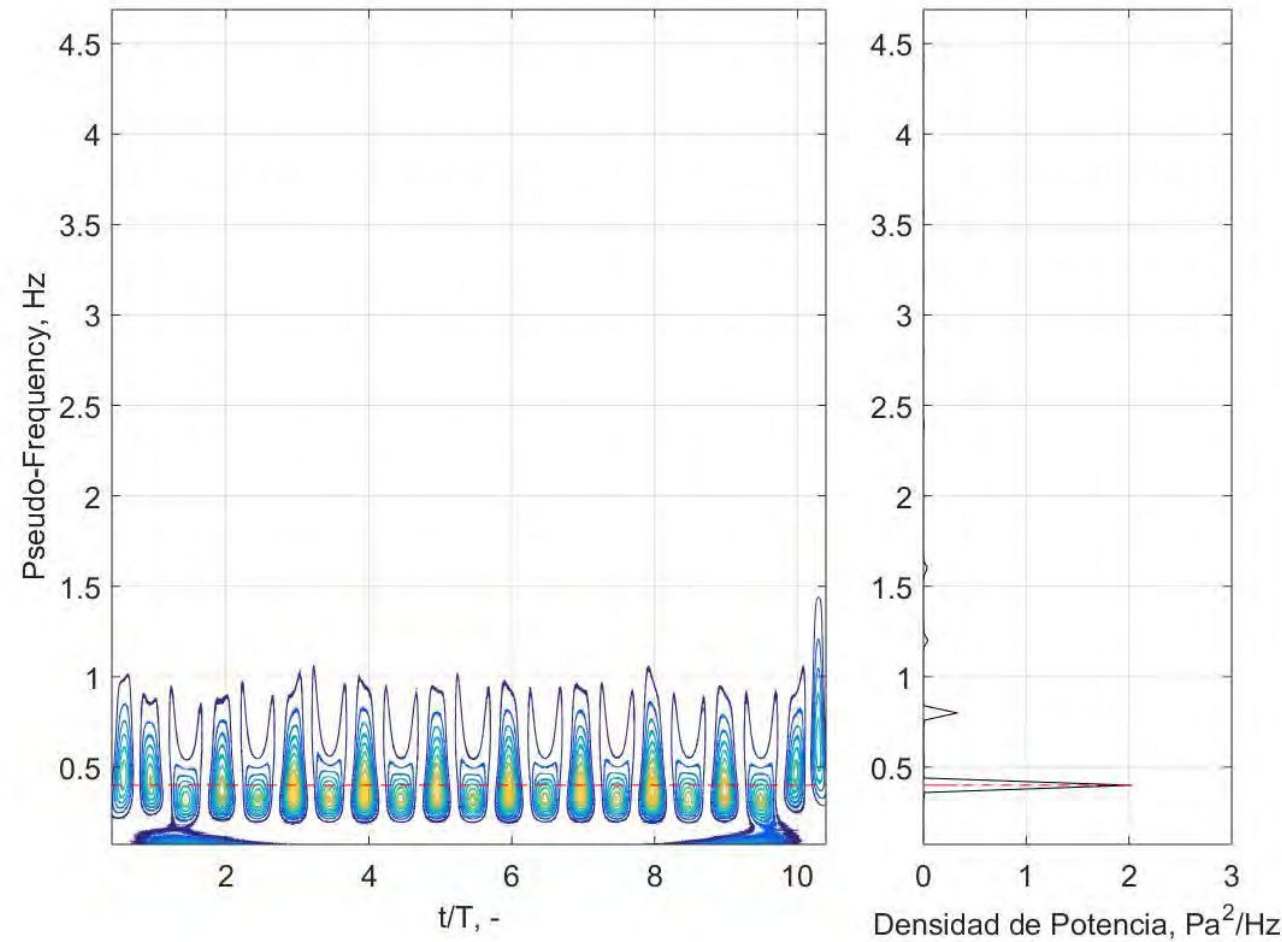

Figura 3.83. Mapa de Wavelets y Densidad de potencia. Reynolds 46.000, $\mathrm{k}=\mathbf{0 , 0 1 5}$ para ubicación media cuerda aguas abajo del borde de fuga y ambas intensidades de turbulencia. Modelo Alar: Perfil SD8020 
U: $8 \mathrm{~m} / \mathrm{s}$ - k: 0.03 - Grilla 0 - Ubicación: 0.5C
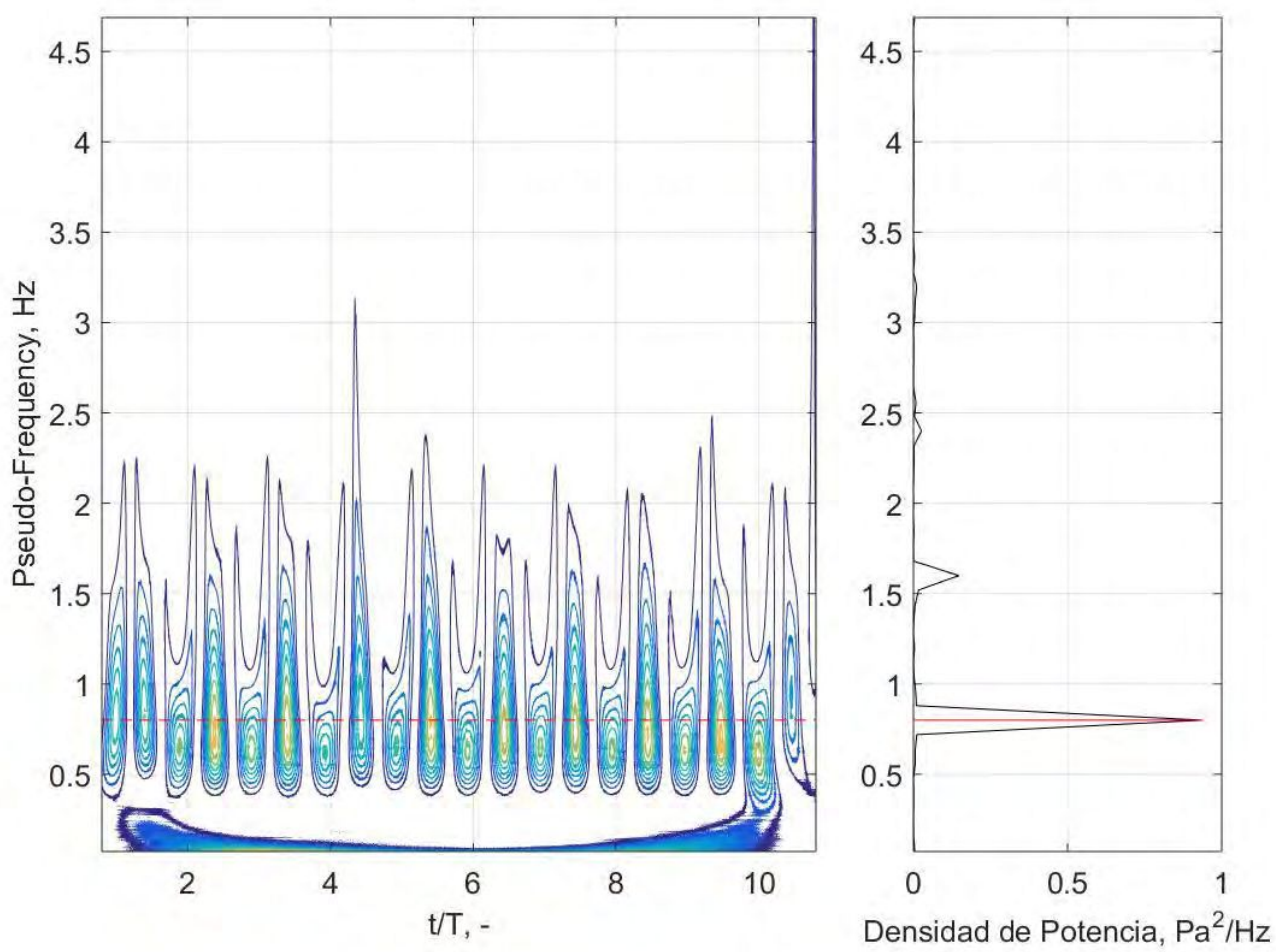

U: 8 m/s - k: 0.03 - Grilla 15 - Ubicación: 0.5C
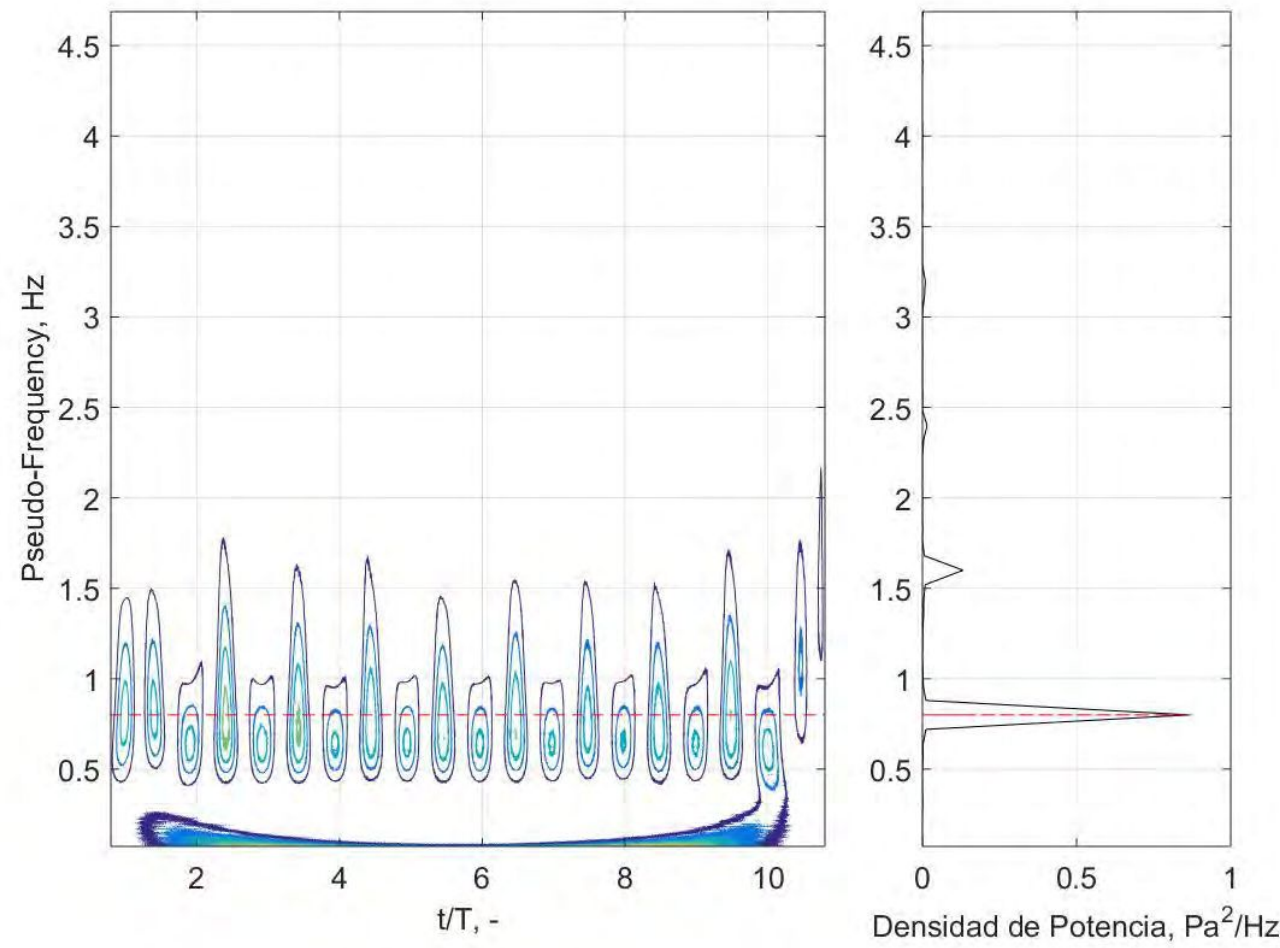

Figura 3.84. Mapa de Wavelets y Densidad de potencia. Reynolds 46.000, $\mathrm{k}=\mathbf{0 , 0 3}$ para ubicación media cuerda aguas abajo del borde de fuga y ambas intensidades de turbulencia. Modelo Alar: Perfil SD8020 
U: $10 \mathrm{~m} / \mathrm{s}$ - k: 0.015 - Grilla 0 - Ubicación: 0.5C
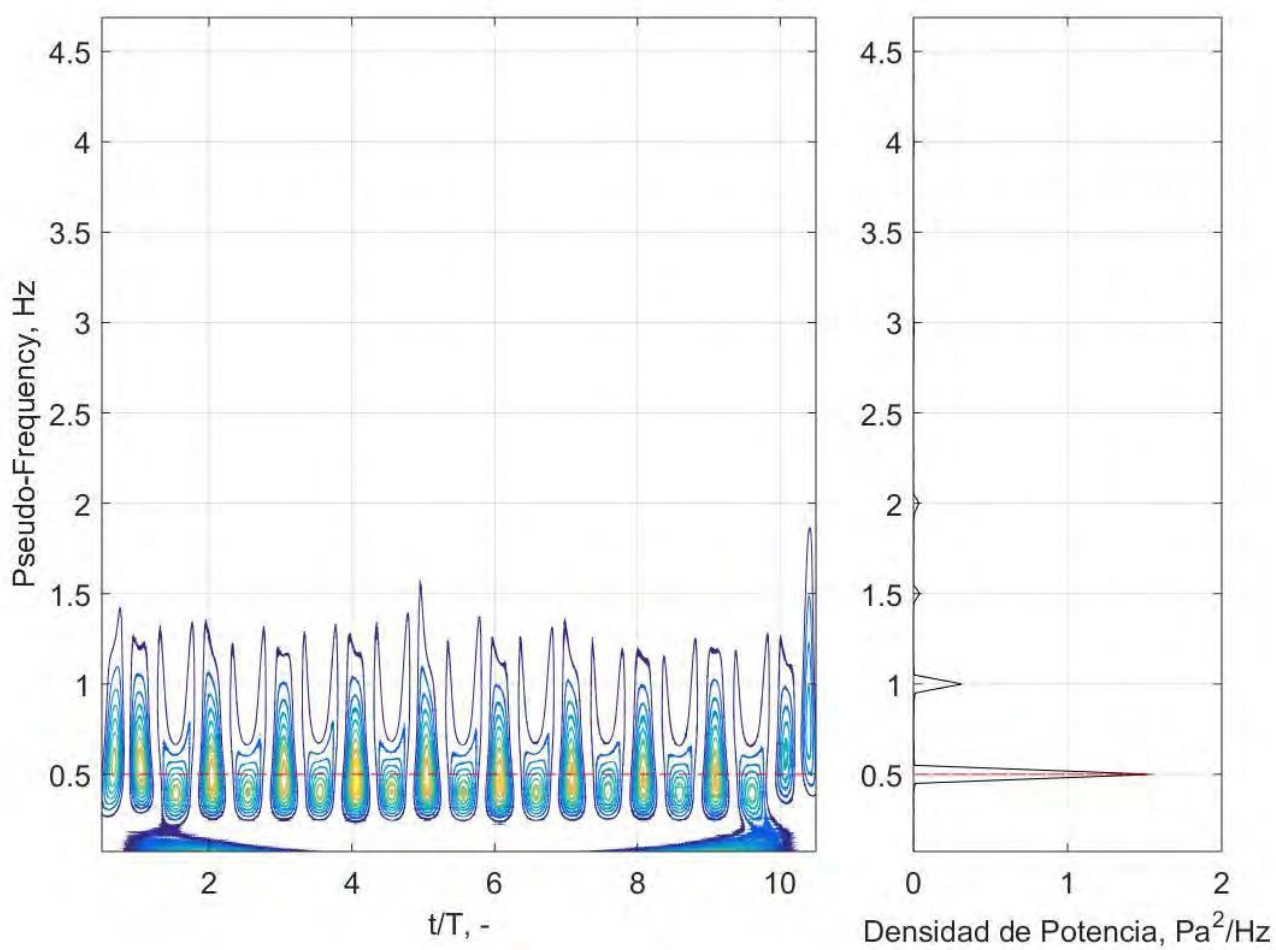

U: 10 m/s - k: 0.015 - Grilla 15 - Ubicación: 0.5C
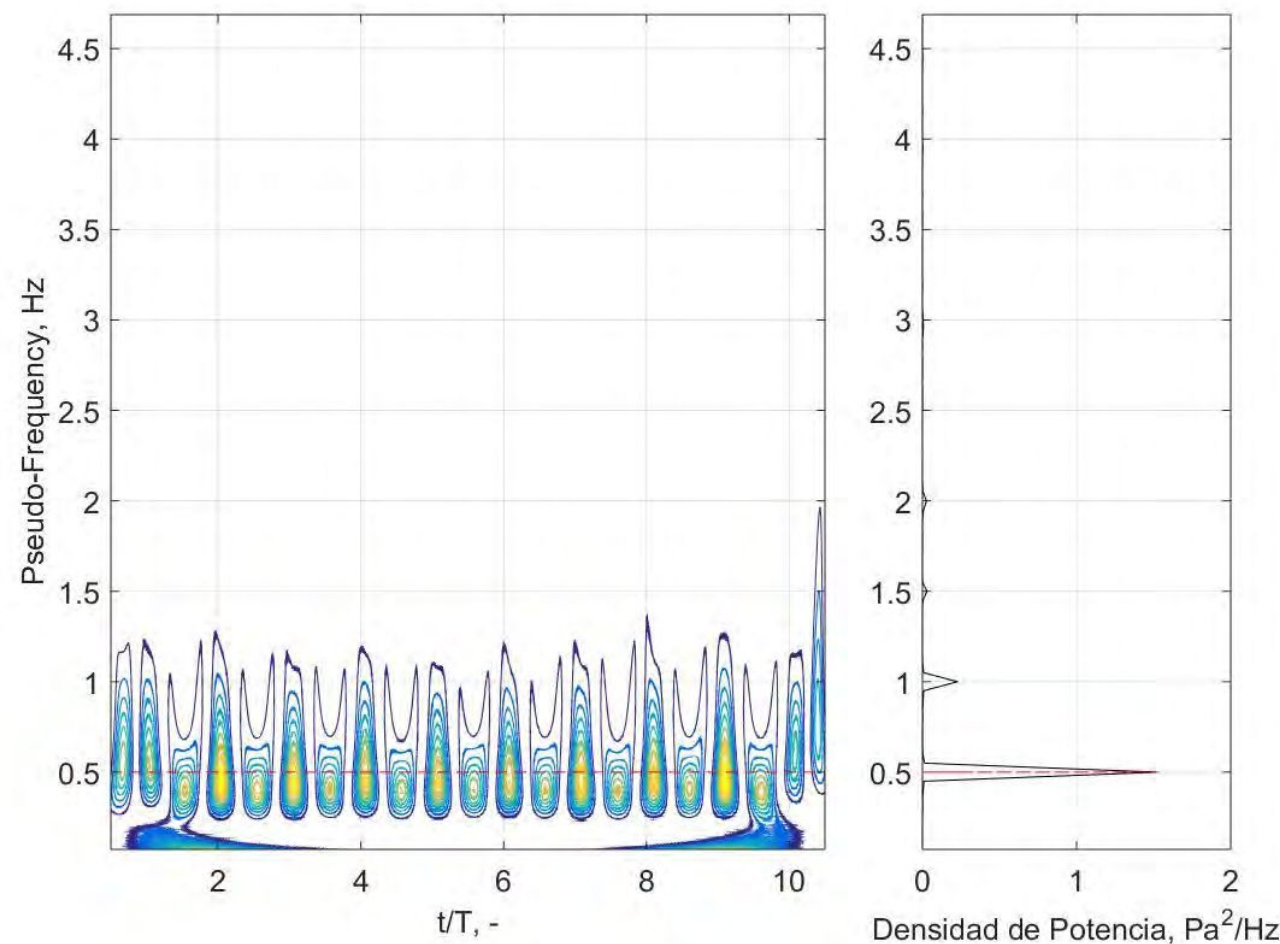

Figura 3.85. Mapa de Wavelets y Densidad de potencia. Reynolds 57.000, $\mathrm{k}=\mathbf{0 , 0 1 5}$ para ubicación media cuerda aguas abajo del borde de fuga y ambas intensidades de turbulencia. Modelo Alar: Perfil SD8020 


\subsubsection{Condiciones dinámicas en perfil SD8020}

En la Figura 3.87 se puede observar una secuencia de imágenes extraídas de la filmación de la visualización mediante la técnica de inyección de humo para el caso de rampa continua con frecuencia reducida $\mathrm{k}=0,025$. En la misma, se dividió la secuencia en dos columnas, siendo la columna de la izquierda la carrera ascendente (incremento en el ángulo de ataque), mientras que la columna de la derecha es la carrera descendente.

En dicha secuencia de imágenes se puede observar cómo, en la carrera ascendente, el flujo se mantiene pegado a la superficie del perfil hasta alcanzar el ángulo máximo de $15^{\circ}$, donde se desprende un vórtice desde el borde de ataque y el mismo comienza a viajar aguas abajo. Si se realiza un seguimiento del vórtice, se puede encontrar que el mismo se desplaza a una velocidad aproximada de $1,6 \mathrm{~m} / \mathrm{s}$, lo que corresponde con una velocidad equivalente al $40 \%$ de la velocidad de la corriente libre. Esta velocidad fue obtenida de forma aproximada, considerando el desplazamiento del centro del vórtice cuadro a cuadro, y conociendo el tiempo relativo entre cada captura. De todas formas, este resultado se condice con la teoría de Wagner, que demuestra que los vórtices desprendidos en borde de ataque se mueven a una velocidad del $50 \%$ de la corriente libre. Este resultado también demostrado mediante técnicas de PIV en las referencias TR-AVT-202, aunque con una dispersión entre los valores obtenidos. Al observar el mapa de Wavelets de dicha estación, Figura 3.82, se puede determinar que los vórtices se producen para tiempos adimensionales múltiplos de 0,5 y de $1 \mathrm{t} / \mathrm{T}$, coincidente con lo observado en la secuencia de imágenes con humo.

$\mathrm{Al}$ analizar la secuencia de imágenes en la carrera descendente, ocurre un proceso similar, donde durante el movimiento del ala el flujo se encuentra pegado, hasta casi el final de la carrera donde se desprende un vórtice en el borde de ataque y luego el flujo se vuelve a adherir, producto de que en esta condición el ángulo de ataque es cercano a $0^{\circ}$. 
"Estudio aerodinámico experimental en flujo turbulento de bajo Reynolds sobre alas con movimiento de cabeceo"

$0^{\circ}$

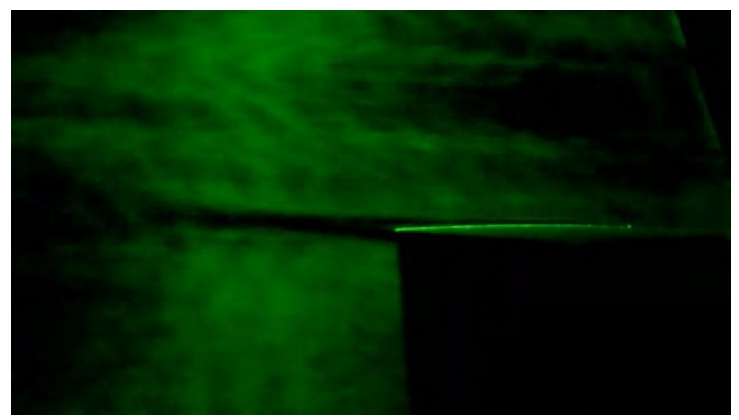

$5^{\circ}$

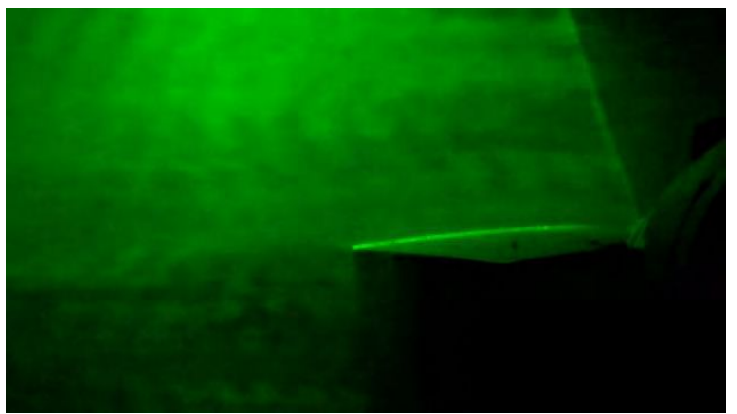

$10^{\circ}$

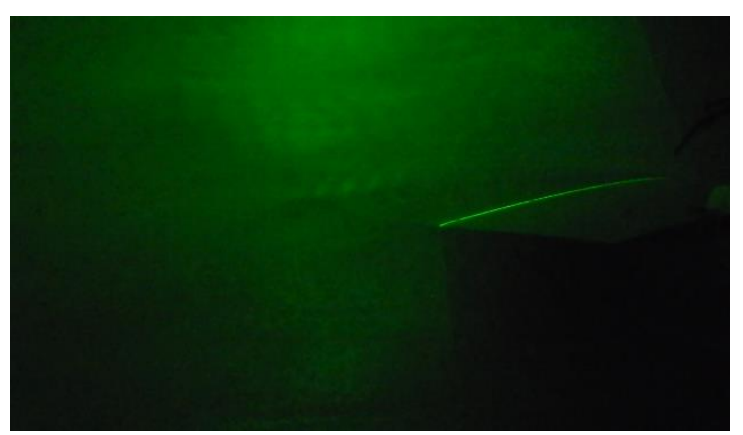

$12^{\circ}$

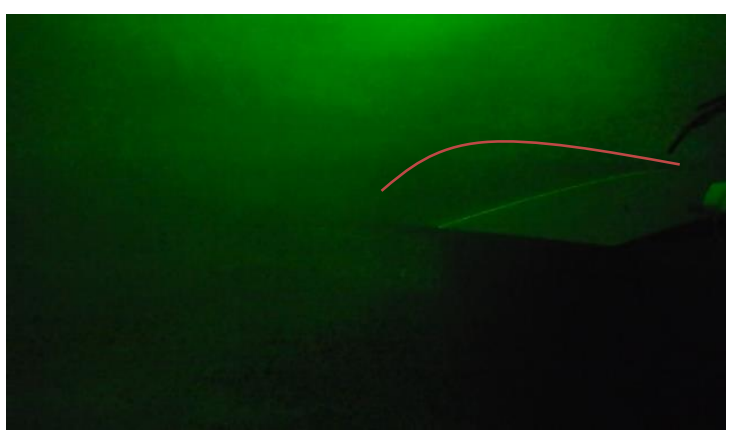

$15^{\circ}$

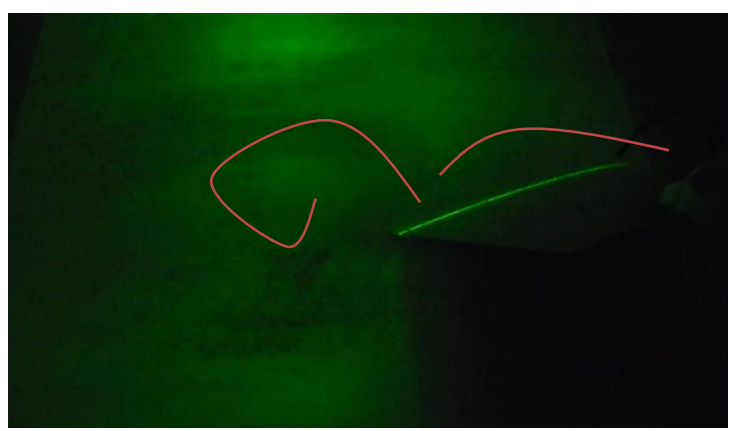

Figura 3.86. Visualización con humo para condición estática del ángulo de ataque 

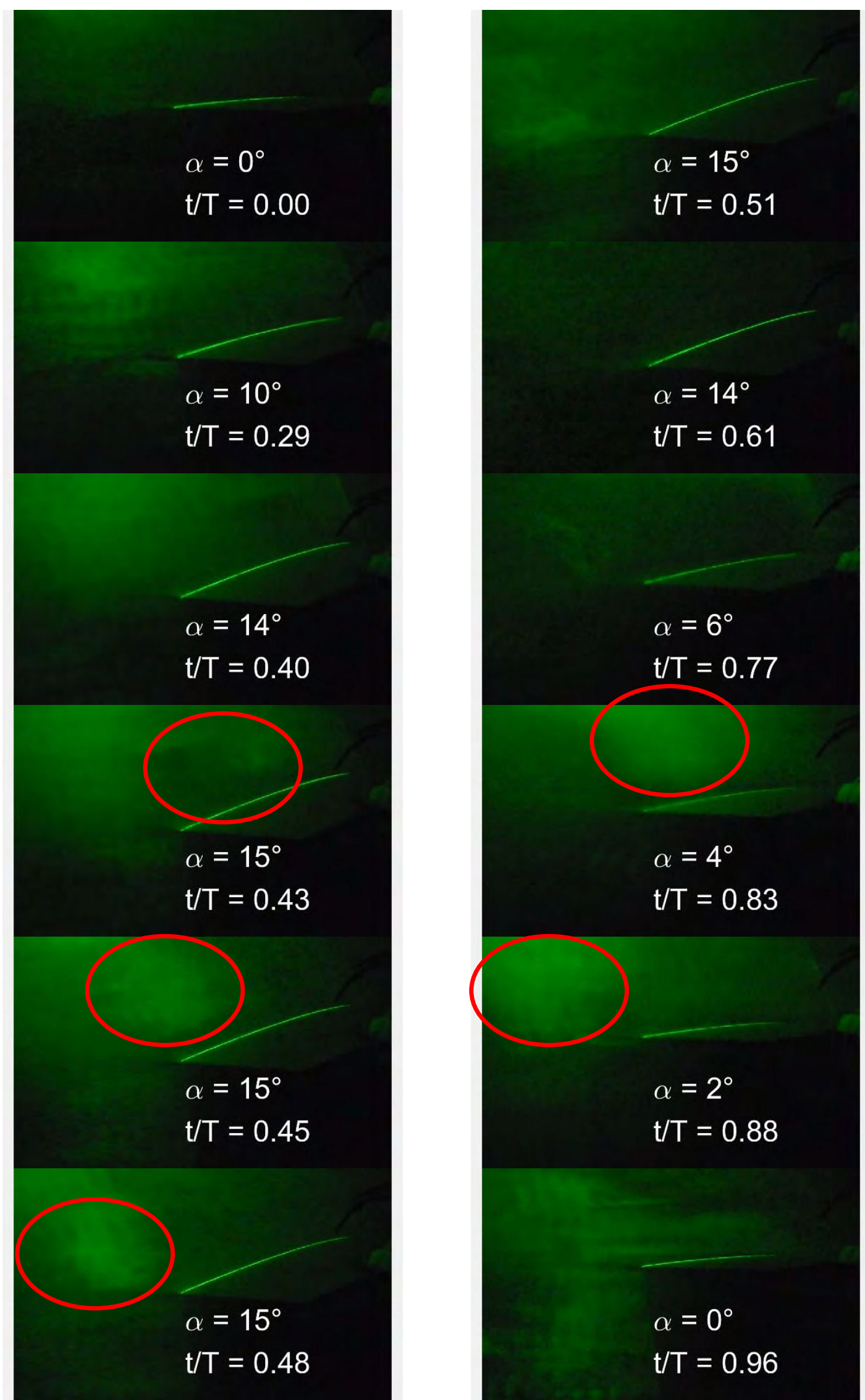

Figura 3.87. Secuencia de imágenes para caso dinámico con frecuencia reducida $\mathbf{k = 0 . 0 2 5}$. 


\section{"Estudio aerodinámico experimental en flujo turbulento de bajo Reynolds sobre alas con movimiento de cabeceo"}

\section{CAPÍTULO 4: Conclusiones y discusión}

Dada la problemática encontrada, y la falta de información sobre la influencia de la turbulencia en las cargas aerodinámicas y el campo de flujo en condiciones aerodinámicas no estacionarias de movimiento de cabeceo, se realizaron ensayos en túnel de viento para dos modelos alares, una placa plana y un perfil SeligDonovan 8020 , en dos condiciones de flujo incidente diferentes.

Para poder llevar a cabo dichos ensayos, se construyeron en primera instancia dos modelos alares, de cuerda $10 \mathrm{~cm}$ y envergadura 39,8 cm para que el mismo quede comprendido de pared a pared dentro del túnel de viento. El primer modelo fue realizado con una placa plana de aluminio de $3 \mathrm{~mm}$ de espesor $(\mathrm{t} / \mathrm{c}=3 \%)$, mientras que el segundo fue construido en fibra de vidrio con un perfil simétrico de 10\% de espesor Selig-Donovan 8020 . Ambos presentan la posibilidad de vincularse a una balanza aerodinámica de dos componentes, mediante un acople especialmente diseñado y construido. Ambos modelos permiten que el acople se vincule en tres posiciones diferentes, pudiendo así modificar el eje de rotación del modelo, aunque para este trabajo solo se utilizó con el eje de rotación localizado en la mitad de la cuerda. Además, se construyó un soporte exterior al túnel de viento, así como un mecanismo capaz de imprimirle movimiento de cabeceo puro al modelo alar que se extiende de pared a pared del túnel de viento. Para poder realizar dicho movimiento se desarrolló un código informático que, en función de un movimiento predefinido, y la posición actual del modelo alar, realiza el movimiento del ala. El movimiento elegido, fue un movimiento tipo rampa ida y vuelta, pero con una función de suavizado para eliminar los cambios bruscos en velocidad de rotación, por ende, minimizar las aceleraciones, las cuales generan cargas inerciales no deseadas. Este mecanismo fue ensayado, y validado en su repetitividad, así como la determinación de la máxima frecuencia de oscilación capaz de realizar.

Se realizaron los ensayos comenzando con la caracterización del flujo incidente utilizando anemometría de hilo caliente. A partir de una configuración base, o limpia, se implementó una grilla generadora de turbulencia para incrementar la turbulencia en la sección de prueba. En la condición base, o limpia, se determinó que la mínima intensidad de turbulencia que posee el flujo libre en la sección de prueba es de $0,3 \sim 0,4 \%$, mientras que al implementar la grilla generadora de turbulencia la intensidad aumenta a $0,6 \sim 0,7 \%$, para la componente vertical de velocidad. En el análisis de la componente longitudinal, se puede definir una intensidad de turbulencia de $0,4 \%$ (aunque no completamente uniforme en toda la sección de prueba) cuando no está presenta la grilla generadora de turbulencia, y al colocar esta, se incrementa la intensidad de turbulencia a un valor cercano a $0,8 \sim 0,9 \%$. Al analizar las escalas integrales turbulentas, utilizando el criterio de $1 / \mathrm{e}$ en la auto correlación, se determinó que para el caso sin grilla, las escalas no están definidas para la componente longitudinal, tomando valores entre 20 y $80 \mathrm{~mm}$ ( 20 a $80 \%$ de la cuerda), pero en la componente vertical estas escalas oscilan entre un valor de 25 a $60 \%$ de la cuerda del modelo alar. Para el caso donde se agregó una grilla generadora de turbulencia, las escalas se uniformizaron para las distintas velocidades en valores en torno a 15 20 mm, siendo un tamaño característico muy similar al tamaño de la grilla $(15 \mathrm{~mm})$. En comparación entonces, tenemos un aumento de la turbulencia en casi el doble entre el caso sin grilla a un caso con grilla, especialmente en la componente vertical, mientras que al analizar las escalas integrales de turbulencia, en la componente vertical se observa un decrecimiento de estas, aunque para la componente longitudinal de la velocidad, solo se observa una homogenización de estas escalas. Otro punto importante a denotar, es que al analizar la señal de velocidad en el campo de frecuencias, el colocar la grilla genera un aumento en la densidad de potencia espectral, para valores de frecuencia mayores a $10 \mathrm{~Hz}$ en la componente vertical para todas las velocidades, mientras que para la componente longitudinal, solo se observa un cambio en el caso de $4 \mathrm{~m} / \mathrm{s}$.

Conociendo las cualidades turbulentas del flujo incidente, se procedió a cuantificar las cargas aerodinámicas, concentrándonos en la sustentación generada por los modelos alares, tanto para condiciones estáticas, como al generar el movimiento planteado. En primera instancia se estudiaron las características 


\section{“Conclusiones y discusión”}

aerodinámicas del modelo alar con placa plana y borde de ataque y borde de fuga romo, considerando como caso de estudio de validación, el movimiento de rampa ascendente suavizada dado que se contaba con suficiente información para poder comparar resultados. Si bien las velocidades de rotación implementadas no eran idénticas a las de la referencia, se logró capturar la tendencia de crecimiento del coeficiente de sustentación según lo estipulado en los resultados de los autores de referencia, así como la comparación con el método teórico implementado. De esta forma, se validó la metodología de ensayo, principalmente la de tarado dinámico de las cargas, mediante el uso de un elemento másico equivalente que minimice los efectos aerodinámicos. En este movimiento, además se realizó la comparación del efecto del incremento de la turbulencia, encontrando que el efecto de la misma genera un cambio de tendencia en el coeficiente de sustentación, en la zona de pérdida del ala, de una forma similar al efecto en condiciones estáticas. Es decir, mientras el flujo se mantiene adherido al ala, no se observan diferencias en la carga aerodinámica, pero donde se presentan desprendimientos, la turbulencia genera un efecto sobre esta, de igual manera en condición estática que en condición dinámica. Lo interesante a destacar, en este modelo de placa plana es que el efecto generado por la turbulencia fue contrario al esperado, dado que contradice lo expuesto por otros autores para condiciones estáticas. En las mediciones realizadas, al incrementar la intensidad de turbulencia, se encontró un valor menor de máximo coeficiente de sustentación, cuando en la bibliografía sobre este fenómeno, otros autores encontraron que el incremento en la intensidad de turbulencia genera un incremento en la sustentación máxima. La mayor diferencia entre los resultados expuestos por otros autores y el modelo ensayado en este trabajo radica en la geometría del borde de ataque y borde de fuga. Para la condición ensayada los bordes no fueron redondeados ni se realizó ningún tratamiento de suavizado; es conocido que, principalmente para condiciones de bajo número de Reynolds como las ensayadas, la geometría del radio de borde de ataque determina la distribución de presiones sobre la cara a succión del ala, así como también el ángulo de salida del borde de fuga, aunque en mucho menor medida. En este modelo, y estos números de Reynolds, por lo tanto, los desprendimientos serán abruptos y desde el borde de ataque, mientras que en perfiles aerodinámicos el punto de transición dependerá del radio de borde de ataque, por lo que la forma de la capa límite sobre el perfil es altamente influenciada por la turbulencia, no así en la placa plana ensayada.

Con la metodología de ensayo validada, y ya con un entendimiento del fenómeno dinámico que ocurre con los desprendimientos de borde de ataque y borde de fuga cuando un ala tiene un movimiento de cabeceo, así como el efecto de la turbulencia incidente, se procedió a ensayar la placa plana en condiciones de movimiento oscilante continuo. Nuevamente se escogió un movimiento de rampa, para poder tener tramos de velocidad constante, por lo tanto aceleración nula durante esa fase del movimiento. Los resultados encontrados muestran un comportamiento similar al encontrado para el caso de rampa ascendente únicamente. Al analizar los resultados, y comparar la carga desarrollada de forma dinámica, respecto al caso estático, se puede observar como claramente se retrasa la entrada en pérdida del ala hasta que el movimiento culmina, para la máxima frecuencia reducida ensayada $(\mathrm{k}=0,06)$, mientras que para las frecuencias reducidas menores, la entrada en pérdida ocurre para un valor de ángulo de ataque similar al de entrada en pérdida para condiciones estáticas. Estos resultados concuerdan con los resultados expuestos por otros autores, los cuales afirman que el incremento en la frecuencia reducida produce efectos que retrasan la entrada en pérdida del ala, pero cuando las velocidades de rotación no son lo suficientemente elevadas, el efecto es despreciable. A su vez, al realizar los ensayos para tres números de Reynolds diferentes, se verificó la posible influencia de este último, sin encontrar efecto alguno, por lo menos para el rango analizado. Estos resultados también se condicen con los expuestos por otros autores, que afirman que el Reynolds no genera influencia, para el rango de bajos números de Reynolds, salvo cuando este es menor a 100, donde los efectos de la viscosidad del fluido determinan la no formación de vórtice de borde de ataque y de fuga. Se repitieron los ensayos para la condición de flujo turbulento incidente, con intensidad cercana al $1 \%$, encontrando resultados muy similares a los hallados para el movimiento de tipo rampa. El efecto principal radica en la disminución del coeficiente máximo de sustentación, resultado encontrado para la condición estática también. No se observan modificaciones en el patrón de histéresis de la 


\section{"Estudio aerodinámico experimental en flujo turbulento de bajo Reynolds sobre alas con movimiento de cabeceo"}

carga desarrollada producto de la turbulencia, aunque los resultados muestran que el efecto de la turbulencia solo es apreciable para la condición de Reynolds 23.000, mientras que para valores mayores de número de Reynolds, el efecto de la turbulencia es despreciable. Aquí radican diferentes puntos de discusión sobre el efecto de la turbulencia, y la importancia de la componente inercial del fluido, respecto a su componente viscosa, disipativa. Otro punto discutido, radica en la energía en frecuencias del fluido para las distintas velocidades ensayadas, donde para la mínima velocidad, por ende mínimo valor de número de Reynolds, se encontró mayor energía en el flujo turbulento en el rango de frecuencias bajo (cercanas a $10 \mathrm{~Hz}$ ) en la componente longitudinal de la velocidad, mientras que para las demás velocidades, si bien la turbulencia es modificada, no se ve un cambio en la densidad de energía espectral, para esta componente. Esta diferencia de energía puede estar generando modificaciones en el patrón vorticoso del ala, mientras que cuando el flujo tiene menor energía no es capaz de modificarlo, lo cual justifica el efecto de variación en las cargas aerodinámicas únicamente para el menor número de Reynolds.

Luego de las mediciones y análisis realizado en placa plana, se realizó el ensayo en un modelo alar construido en fibra de vidrio utilizando un perfil aerodinámico de bajo número de Reynolds, SD8020. Este perfil cuenta con la particularidad de ser un perfil simétrico, con 10\% máximo de espesor, es decir, se lo puede considerar un perfil delgado, pero que a bajos ángulos de ataque presenta una burbuja de recirculación lo cual genera una curva de sustentación que no es lineal con el ángulo de ataque. En las mediciones realizadas, para una condición estática del ángulo de ataque, la curva de sustentación en función del ángulo de ataque muestra la no linealidad encontrada por Selig en sus ensayos en flujo laminar, aunque en los casos ensayados en este trabajo, producto de la turbulencia incidente, la forma de "s" en la curva no se encuentra tan marcada. Esto coincide con resultados obtenidos mediante simulación numérica utilizando el software XFoil. Al realizar la comparación entre una turbulencia incidente del 0,4\% y del 0,7\%, para este perfil se observa un retraso en la entrada en pérdida, así como un aumento en el coeficiente de sustentación máximo, apoyando la hipótesis de importancia del borde de ataque del perfil en la influencia de la turbulencia sobre la sustentación que fue expresada anteriormente. Es decir, para el ensayo realizado con el perfil aerodinámico, se confirma los resultados expresados por otros autores, aunque dados los resultados encontrados en placa plana, se enfatiza en la importancia del radio de borde de ataque para poder realizar afirmaciones sobre la influencia de la turbulencia, por lo menos en condiciones de bajo número de Reynolds. Al proceder a realizar los ensayos en condiciones no estacionarias, con el mismo movimiento de rampa ascendente y descendente, se puede observar como nuevamente el patrón de influencia de la turbulencia es muy similar al encontrado en placa plana. Es decir, la influencia de la turbulencia sigue la misma tendencia que para el caso estacionario, en este caso, incrementando el coeficiente máximo de sustentación encontrado, lo que se condice con un retraso en la entrada en pérdida del ala. Un punto importante a denotar es que los resultados hallados para el perfil aerodinámico, en función de la intensidad de la turbulencia, no se modifican en función del número de Reynolds ensayado, como ocurrió para la placa plana. Esto refuta la hipótesis de la diferencia en la energía del flujo presente a bajas frecuencias para bajas velocidades, aunque puede suceder que la energía necesaria para modificar la capa límite en el perfil aerodinámico es menor a la necesaria en la placa plana, por lo que para toda la energía en el flujo, produce modificaciones en la carga aerodinámica. Otro punto observado en las comparaciones de cargas aerodinámicas con menor y mayor intensidad de turbulencia es que las diferencias encontradas son principalmente para el coeficiente de sustentación máximo, asociado a la carrera ascendente, mientras que en la zona de carrera descendente no se observan modificaciones importantes en la fuerza desarrollada. A su vez, tras realizar un análisis en la histéresis de la curva sustentación en función del ángulo de ataque, se encontró que esta está directamente relacionada con el máximo coeficiente de sustentación, manteniéndose aproximadamente constante la relación entre el valor máximo del coeficiente, con la histéresis del movimiento, cuando se modifica la turbulencia. No así, con el incremento de la frecuencia reducida, se encuentra que esta relación aumenta también, como era de esperar, producto de los fenómenos no estacionarios que son intensificados. 
Luego del análisis realizado con las cargas aerodinámicas, se procedió a realizar un análisis de la estela del ala. Para ello se construyó un rack de presiones, compuesto de 31 tomas de presión, las cuales fueron espaciadas de forma tal de tener mayor discretización en la zona próxima al ala, donde se espera tener mayor gradiente en la distribución de presiones. Luego de realizar la validación de las mediciones por el instrumento desarrollado, se procedió a realizar un análisis utilizando diferentes técnicas estadísticas sobre la estela, principalmente para la condición de ensayo dinámico. En primera instancia se realizó una correlación cruzada entre el sensor localizado a la altura del eje de rotación del ala, con respecto a los sensores ubicados en diferentes alturas para identificar el tamaño general de la estela. En función de los resultados, se determinó que el tamaño de la estela es de aproximadamente el valor de media cuerda, y para el caso de placa plana esta se encuentra centrada con respecto al eje de rotación, mientras que para el perfil SD8020, la estela se encuentra levemente desplazada en la vertical hacia el lado de succión del ala (para la carrera ascendente). Este resultado fue independiente del número de Reynolds ensayado, así como de la frecuencia reducida. Al cambiar la intensidad de turbulencia, se encontró, para la placa plana, que nuevamente la influencia de la turbulencia se puede observar para valores bajos de número de Reynolds, mientras que para los valores mayores, la influencia de la turbulencia no se puede apreciar. En el caso de mínimo valor de Reynolds, el incremento de turbulencia produce una mayor difusión en la estela, observado en los valores de correlación de las señales, que se ve disminuido al incrementar la turbulencia para este caso. Al realizar el mismo análisis a una distancia mayor (una cuerda aguas abajo, en vez de media cuerda), se observa un decaimiento en los valores de correlación encontrados, esperado dado que al encontrarnos en una estación más alejada, la estela es difuminada por el efecto de disipación de la turbulencia. Otro análisis realizado fue el de correlación cruzada para dos estaciones de medición diferentes, encontrando así un desplazamiento vertical del vórtice de borde de ataque, en un valor del 10\% de la cuerda, hacia arriba, correspondiente al lado de succión del ala en la carrear ascendente, tanto para la placa plana como para el perfil aerodinámico. Finalmente, se realizó un estudio en frecuencias utilizando espectro de Fourier y Wavelets. Con los mapas de wavelets, para el sensor localizado a la misma altura que el eje de rotación, se puede observar los fenómenos periódicos concentrados en torno a la frecuencia de movimiento del ala. Además, se puede observar como al incrementar la frecuencia reducida del movimiento, las componentes en frecuencias en la estela dejan de ser tan cercanas al movimiento involucrando un rango mayor de frecuencias, en los vórtices asociados. Al incrementar la intensidad de turbulencia se puede observar un comportamiento diferente entre la placa plana y el perfil aerodinámico nuevamente. Para la placa plana se observa una reducción en la densidad de potencia al incrementar la intensidad de turbulencia, mientras que para el perfil aerodinámico, estas diferencias no son apreciables.

Por último, se realizaron visualizaciones de flujo mediante inyección de humo en la corriente libre y la ayuda de un plano láser. En las visualizaciones realizadas para ángulos de ataque estáticos, el comportamiento observado de flujo se condice con la curva de sustentación para el perfil aerodinámico SD8020, observándose un desprendimiento de flujo en $12^{\circ}$ de ángulo de ataque, mientras que para $0^{\circ}$ se aprecia una separación de flujo en el borde de fuga que puede estar relacionado con el comportamiento no lineal de la curva de sustentación. Al realizar las visualizaciones en condiciones dinámicas, para la máxima velocidad de rotación se pudo capturar el vórtice desprendido en el borde de ataque y seguirlo conforme aumenta el tiempo de filmación, aunque producto de la imposibilidad de realizar una filmación perpendicular al flujo, la determinación de la distancia recorrida no pudo ser determinada con la precisión suficiente, con lo cual se decidió no realizar dichas estimaciones en estas visualizaciones.

En líneas generales, y a modo de resumen, se realizaron mediciones de dos modelos alares, provistos de diferencias geométricas en su distribución de espesor y principalmente, la geometría del borde de ataque y fuga, para condiciones no estacionarias en dos configuraciones de flujo turbulento. Las mediciones muestran cambios en el comportamiento de las cargas aerodinámicas cuando el flujo incidente es modificado, siguiendo un patrón muy similar al encontrado para ensayos en condiciones estáticas, notándose el efecto 
principal de la turbulencia en la zona de ángulos de ataque mayores al ángulo de entrada en pérdida estático. Esta situación permite poder realizar una extrapolación de los resultados encontrados para condición estática, en la condición no estacionaria, teniendo en cuenta que igualmente se generará el ciclo de histéresis, siendo el cociente entre la histéresis y el coeficiente de sustentación máximo independiente de la turbulencia incidente. Además, se encontró que el efecto de la turbulencia en las cargas, así como en la estela, es altamente dependiente de la geometría del ala, principalmente del radio de borde de ataque, por lo que no se pueden generalizar los resultados encontrados para cualquier perfil utilizado. Este resultado se condice con mediciones de perfiles aerodinámicos en condiciones de bajo número de Reynolds. Es importante destacar entonces, que si se está pensando en realizar la determinación de cargas aerodinámicas para el diseño de un vehículo aéreo no tripulado de pequeña escala, con la capacidad de mover sus alas, por lo menos para el movimiento de cabeceo, que está altamente relacionado con la generación del vórtice de borde de ataque, el efecto de la influencia sigue una tendencia similar al encontrado en condiciones estáticas, con lo cual se puede aproximar el incremento (o decremento) del coeficiente máximo de sustentación en función de lo hallado para condiciones estáticas. A su vez, se encontraron, diferencias importantes en las estructuras de la estela cuando la intensidad de turbulencia fue modificada, para una placa plana, pero no así para el caso del perfil SD8020, lo que reafirma la dependencia del comportamiento aerodinámica en la geometría del perfil utilizado. 


\section{Anexo I: Conceptos de Aerodinámica General}

Desde el punto de vista de la mecánica de los fluidos, la materia puede tener dos estados: sólido o fluido. La diferencia entre ambos, es su respuesta ante un esfuerzo de corte o tangencial. Un sólido puede absorber y resistir dicho esfuerzo mediante una deformación estática, mientras que el fluido no. Por esta definición, un fluido, que puede ser líquido o gaseoso, ante la acción de una tensión, no puede hacer otra cosa más que deformarse continuamente.

La aerodinámica, es la rama de la mecánica de los fluidos que estudia las leyes físicas y aplicaciones que gobiernan el desplazamiento de un cuerpo en el seno de un fluido. En particular, para este trabajo, es de interés el entendimiento del comportamiento del fluido cuando atraviesa un ala, cuando esta posee un movimiento característico, y las fuerzas que se desarrollan en dichas condiciones.

Para calcular la fuerza aerodinámica que actúa sobre el ala, básicamente se debe evaluar la variación en el tiempo de la cantidad de movimiento del fluido al atravesar el objeto, en este caso, el ala. Es decir, para poder generar una fuerza de sustentación, por ejemplo, el objeto debe poder generar un cambio en la cantidad de movimiento en el fluido, de forma tal que la reacción sobre el cuerpo sea una fuerza normal a la velocidad del fluido, en el sentido deseado. Cualquier cuerpo de cualquier forma que se mueva en el seno de un fluido por lo menos tendrá que apartar al fluido para poder pasar, generando variaciones de velocidad en el fluido que rodea a ese cuerpo. Cuando por un cierto lugar del espacio ocupado por un fluido pasa un cuerpo, las velocidades y las presiones de ese lugar serán obviamente diferentes de lo que eran antes de la llegada del cuerpo. Observando diversas visualizaciones de flujo apreciaremos conformaciones fluidodinámicas rectilíneas, curvas, permanentes, oscilantes de tipo periódico o aleatorio, remolinos de formas muy diversas, cilíndricos abiertos o cerrados, remolinos cónicos, elípticos, anillos vorticosos, etc. Las fuerzas y en particular la sustentación que aparecerán debido a la variación de cantidad de movimiento producida por estos flujos serán determinadas por la esencia de cada uno de estos movimientos. Muchos de los movimientos mencionados, en particular aquellos que pueden ser identificados (aunque sea aproximadamente) en el seno de un fluido, como los remolinos mencionados, son también conocidos bajo el nombre de estructuras fluidodinámicas. El tipo de estructura fluidodinámica más conocido es la estructura vorticosa; es decir una distribución de velocidades que tiene aspectos de vórtice o remolino.

Entonces, en aerodinámica resulta importante analizar diferentes formas de objetos que sean capaces de modificar la cantidad de movimiento del fluido, para poder generar las fuerzas necesarias y en el sentido deseado. Al pensar en aeronaves, el sistema alar es el responsable de dicha misión, siendo su sección transversal (perfil aerodinámico), uno de los principales factores para la generación de fuerzas, junto con la forma del ala. Al concentrarnos en el perfil, quién es uno de los principales actores a la hora de generar un cambio en la cantidad de movimiento del ala, de forma tal de generar fuerza sustentación, podemos encontrar distintos parámetros geométricos que lo definen: radio de borde de ataque, espesor máximo, ubicación del máximo espesor, curvatura, ángulo del borde de fuga. La elección del perfil aerodinámico, y por ende sus características geométricas, dependerá de la condición de vuelo a la que esté inmerso. Esta condición queda definida principalmente, por dos números adimensionales de la fluidodinámica: el número de Reynolds y el número de Mach. El número de Reynolds, el cuál es una relación entre las fuerzas inerciales y las fuerzas viscosas, mientras que el número de Mach representa la relación entre la velocidad del fluido y la velocidad del sonido de ese fluido, es decir, demuestra si el fluido se comporta de forma compresible o incompresible. En el rango de bajos números de Mach, el fluido se comporta de forma incompresible, mientras que para valores mayores a 0,2, estos efectos dejan de ser despreciables. El número de Reynolds, determinará el efecto de los esfuerzos viscosos, lo cual está directamente relacionado con la capa límite del perfil. Se entiende como capa límite a la zona cercana a la pared de un objeto, donde el gradiente de velocidades, y por ende de presiones, en la dirección perpendicular 


\section{"Estudio aerodinámico experimental en flujo turbulento de bajo Reynolds sobre alas con movimiento de cabeceo"}

a la superficie es importante. Es decir, es la zona donde se concentran los efectos viscosos, mientras que, por fuera de esta zona, el flujo se puede considerar inercial completamente, despreciándose los efectos viscosos. En la literatura convencional, se considera como bajo número de Reynolds al rango entre 50.000 y 500.000, mientras que para Reynolds mayores a un millón, nos encontramos con características de altos números de Reynolds. En el régimen de bajos números de Reynolds, es decir, bajas velocidades y/o pequeños valores de cuerda, la capa límite del perfil aerodinámico es muy susceptible a los gradientes de presiones, lo cual produce efectos que en otro régimen de operación no ocurre. Omera y Mueller, 1987, demostraron que para condiciones de bajos números de Reynolds se forma una estructura vorticosa, denominada burbuja de separación laminar turbulenta, dentro de la capa límite, donde el flujo se separa, transiciona de laminar a turbulento y se re adhiere a la pared del perfil, modificando la performance del perfil, respecto a si no ocurriese dicha burbuja. Esto produce además, apartamientos del comportamiento lineal que posee el coeficiente de sustentación con respecto al ángulo de ataque, para valores de ángulo de ataque menores al de pérdida, lo cual está relacionado con la separación del flujo de la pared del perfil.

En los diseños convencionales de aeronaves, se utiliza el sistema de ala fija, donde los movimientos y cambios en el ángulo de ataque (ángulo entre el vector velocidad y la cuerda del ala), son de forma cuasiestacionarios. Sin embargo, con los últimos desarrollos, y la miniaturización de los objetos, este tipo de sistemas no son adecuados, producto de las bajas velocidades de vuelo, o las gigantescas superficies alares que se necesitarían para poder volar a baja velocidad. Es allí donde se empiezan a estudiar nuevos sistemas de generación de fuerza de sustentación, mediante sistemas de alas móviles. Ejemplo de ello, son los cuadricópteros o multicópteros que, utilizando el principio de hélice, es decir, un ala rotante, generan la fuerza de sustentación necesaria para que la aeronave pueda volar. Uno de los grandes problemas de este tipo de sistemas, es que para poder modificar su actitud (orientación en el espacio), deben inclinar todo el plano de hélices, para inclinar la fuerza aerodinámica, lo que trae aparejados problemas para el control del vehículo. En estas condiciones es que, imitando al vuelo de las aves, se intenta desarrollar vehículos aéreos con capacidad de aleteo, para poder lograr así la fuerza necesaria de sustentación y empuje, sin la problemática de orientación en actitud de los sistemas de alas rotantes.

\section{Anexo I.I: $\underline{\text { Turbulencia }}$}

Una de las características del viento es que sus parámetros principales varían aleatoriamente a medida que transcurre el tiempo. Los valores instantáneos de las variables como la velocidad, presión, temperatura, densidad, etc., cambian constantemente; este fenómeno se denomina turbulencia. Mediante herramientas estadísticas se pueden obtener valores medios de estas fluctuaciones para caracterizar la turbulencia.

Podemos definir entonces a la velocidad de una partícula para cada intervalo de tiempo como la suma de una velocidad media y una fluctuación:

$$
U(t)=\bar{u}+u^{\prime}
$$

El viento se concibe, pues, como resultado de una corriente con una dada velocidad media que arrastra remolinos de diferente tamaño e intensidad. Los conocimientos modernos sobre flujos turbulentos permiten afirmar que, inmersas en el flujo aleatorio, es posible hallar estructuras coherentes oscilatorias no-aleatorias, es decir, no-turbulentas en el sentido usual de la palabra. Una de las disciplinas experimentales modernas tiene como objetivo la identificación de dichas estructuras en un problema dado.

El conocimiento de las escalas de remolinos presentes, junto con la amplitud y distribución de la energía en el rango de frecuencias altas, medias y bajas, en una dada zona del flujo, contribuye a lo que se denomina caracterización de la estructura fina de la turbulencia. 


\section{Anexo II: Corrección por mediciones en túnel de viento}

Las condiciones del flujo dentro del túnel de viento, claramente, difieren de las condiciones al "aire libre", debido a las restricciones de contorno que se le imponen al flujo. El efecto de los contornos sólidos, producto de las paredes del túnel, influirán en mayor o menor medida en función de la distancia entre el objeto de estudio y estas. Sin embargo, esta no es la única fuente de diferencias que se pueden encontrar entre un ensayo en túnel de viento, respecto a un ensayo en vuelo, si hablamos de una aeronave, por ejemplo. Otra diferencia puede estar dada por las perturbaciones del flujo, dado que pequeñas variaciones pueden generar efectos a nivel de tensiones superficiales y fuerzas totales. Un ejemplo de un flujo no uniforme es la presencia de un gradiente de presión estática longitudinal.

En el caso de ensayos que se pretende tener un flujo bidimensional, es común utilizar la técnica de que el cuerpo ocupe todo el ancho o alto de la sección de prueba. Por ejemplo, si se desea realizar los ensayos para tener las características de un perfil aerodinámico, se construye un ala que tenga una envergadura igual al ancho o alto del túnel, de esta forma, se asemeja a un flujo bidimensional, minimizando los efectos de las puntas del modelo (con las consideraciones extras que hay que tener). Para este caso particular, coincidente con la técnica utilizada en este trabajo, se deben realizar ciertas correcciones en las mediciones, las cuales se pueden clasificar en cuatro tipos: "bouyancy" (flotabilidad), bloqueo sólido, bloqueo por estela y curvatura de las líneas de corriente.

Esta clasificación en cuatro formas de bloqueo mencionadas, son las expuestas en el capítulo "Boundary Conditions I" de Barlow, et al. 1999, pero es una metodología de corrección de ensayos para túnel de viento en condiciones estáticas del modelo emplazado en el túnel de viento. Producto de que no hay desarrollada una metodología para corrección de cargas aerodinámicas en condiciones no estacionarias, se procedió a realizar una verificación de las presiones en la estela en la zona cercana a las paredes superiores e inferiores del túnel de viento, para verificar si se evidencian efectos dinámicos producto del movimiento del modelo alar dentro de la sección de ensayo.

En las condiciones de ensayo utilizadas, hay presente dos configuraciones diferentes. En primera instancia, para el caso de medición de cargas para validación de la metodología de ensayo, el ángulo final del movimiento es de $45^{\circ}$, lo que produce un bloqueo (área ocupada por el modelo respecto al área de la sección del túnel), del $18 \%$. Este valor es mayor al recomendado en la bibliografía $(10 \%)$, pero era necesario realizar dichas mediciones para poder validar la metodología, sabiendo que podía llegar a haber diferencias en los valores de cargas máximas en el ensayo, producto de estos efectos de bloqueo. Dada esta problemática, se decidió para el movimiento de rampa ida y vuelta, el de mayor interés de en este trabajo, limitar el ángulo máximo del movimiento a un valor menor al $10 \%$, pero que sea mayor al ángulo de pérdida estático del perfil, para poder maximizar los efectos de desprendimientos vorticosos. Para estos ensayos, el ángulo máximo fue de $15^{\circ}$, lo que genera un bloqueo de $6,5 \%$, el cuál es menor al $10 \%$ que la bibliografía presenta como límite, y muy cercano al $5 \%$ de bloqueo, que es un valor óptimo para los ensayos en túnel de viento.

Retomando la metodología de correcciones para modelos alares que ocupan todo el ancho (o alto) del túnel de viento, en condiciones estáticas, se desarrollará dicha metodología para cada clasificación de bloqueo.

\section{Anexo II.I: Bouyancy}

En la mayoría de los túneles de viento con sección cerrada, hay presente una variación de la presión a lo largo del eje longitudinal de la sección de prueba, lo que produce un engrosamiento de la capa límite en las paredes del túnel lo que conlleva a una disminución del área efectiva de la sección de prueba. Glauert encontró que la magnitud del gradiente se puede expresar de una forma no dimensional a través de la expresión: 


$$
\frac{d p}{d l}=-\frac{k\left(\frac{\rho}{2}\right) V^{2}}{B}
$$

Donde 1 es la longitud del túnel (ft), p la presión ( $\left(\mathrm{b} / \mathrm{ft}^{2}\right)$ y $\mathrm{B}$ el ancho del túnel (lb). $\mathrm{K}$ es un factor que normalmente ronda entre 0.016 y 0.04 para secciones cuadradas.

Si se considera un ala, normalmente este efecto es despreciable (por su corta dimensión), pero al realizar ensayos en fuselajes o nacelas, el efecto toma cada vez más importancia. La corrección se calcula con la siguiente expresión:

$$
D_{B}=\int_{0}^{l} S(x) p^{\prime}(x) d x
$$

Donde S es el área transversal de la sección, y p' es la derivada del gradiente de presiones, Ecuación ( 2 ). Si el gradiente de presiones es constante a lo largo de la longitud del modelo, la expresión anterior se puede evaluar directamente como el gradiente por el volumen del modelo.

La existencia de una caída en el gradiente de presión estática, implica que la sección de prueba efectiva se está contrayendo, por ende, las líneas de corriente se encuentran cada vez más "juntas". Para adicionar este efecto, Allen y Vincenti demostraron que se puede estimar la diferencia total en la resistencia por este efecto calculándolo de la siguiente manera:

$$
\begin{gathered}
D_{B}=\frac{6 h^{2}}{\pi} \Lambda \sigma \mathrm{p}^{\prime} \\
\Lambda=\frac{16}{\pi} \int_{0}^{l} \frac{y}{c}\left\{\left[1-P\left(\frac{x}{c}\right)\right]\left(1+\frac{d y}{d x}\right)\right\}^{\frac{1}{2}} d\left(\frac{x}{c}\right) \\
\sigma=\frac{\pi^{2}}{48}\left(\frac{c}{h}\right)^{2}
\end{gathered}
$$

Donde, h es la altura del túnel, c es la cuerda del modelo y $\mathrm{P}(\mathrm{x} / \mathrm{c})$ es la distribución simétrica sin curvatura del perfil.

\section{Anexo II.II: Bloque Solido}

La presencia de las paredes del túnel confinando el flujo alrededor del modelo, reduce el área por la cual debe fluir el aire, comparado con la condición de "aire libre", lo que, por el principio de Bernoulli, incrementa la velocidad del aire. Este incremento de velocidad, se lo llama bloqueo sólido, que es función del espesor del modelo, la distribución de espesores y del tamaño del modelo y es independiente de la curvatura.

En la literatura se pueden encontrar distintas formas de calcular este bloqueo $(\varepsilon)$, por ejemplo, Glauert lo estima mediante la expresión:

$$
\epsilon_{s b}=0.822 \lambda_{2} t^{2} / h^{2}
$$

Donde le valor de $\lambda_{2}$ se puede obtener de la Figura II.1 que ha sido extraída de Barlow et al 1999 y la relación $(\mathrm{t} / \mathrm{h})^{2}$ representa el área frontal ocupada por el modelo respecto al área de la sección de pruebas.

Allen y Vincenti, reescribieron la ecuación anterior e introduciendo el valor de $\sigma, y=4 \lambda_{2} \mathrm{t}^{2} / \mathrm{h}^{2}$ obtienen:

$$
\epsilon_{s b}=\lambda \sigma
$$




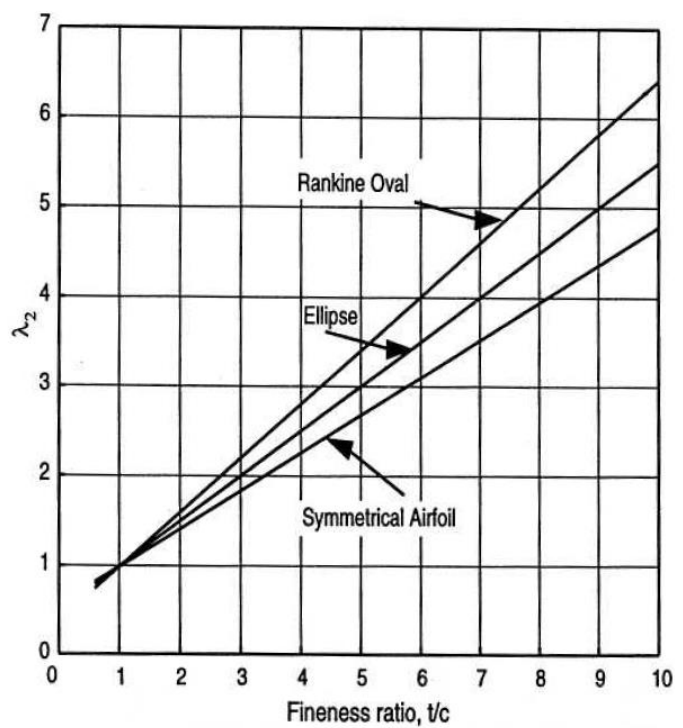

FIGURE 9.16 Shape factors for selected forms.

Figura II.1. Factor de forma para algunas formas seleccionadas. Figura 9.16 extraída de Barlow et al. 1999

\section{Anexo II.III: Bloque por estela}

Todo cuerpo sumergido en un flujo, si no posee algún tipo de control de capa límite por succión generará una estela que tiene menor velocidad media que la de la corriente libre. De acuerdo con la ley de continuidad, la velocidad por fuera de la estela deberá ser mayor a la de la corriente libre para que un volumen constante de fluido pase por la sección transversal. La mayor velocidad en la corriente tendrá por Bernoulli, menor presión, y esto se incrementa a medida que crece la capa límite del modelo, imprimiéndole al modelo un gradiente de presiones que resulta en un incremento de la velocidad local.

Para calcular este efecto, se debe considerar el cambio de velocidades producto de la estela, lo que, aplicando teoría potencial, nos conduce a la siguiente expresión:

$$
\epsilon_{w b}=\frac{\Delta V}{V_{u}}=\tau C_{d u}=\frac{\frac{c}{h}}{4} C_{d u}
$$

En contrapartida, Maskell estudió el mismo efecto, y encontró que la corrección debe realizarse es

$$
\epsilon_{w b}=\frac{\left(\frac{c}{h}\right)}{2} C_{d u}
$$

Para ambos casos, se introduce en el cálculo del bloqueo por estela $\left(\varepsilon_{\mathrm{wb}}\right)$ el uso de una "variable medida", como es el coeficiente de resistencia $\left(\mathrm{C}_{\mathrm{du}}\right)$. El efecto del gradiente por estela, de Allen y Vincenti, que usualmente es muy pequeño, se puede calcular como:

$$
\Delta C_{d, w b}=\lambda \sigma
$$

\section{Anexo II.IV: Curvatura de las líneas de corriente}

La presencia del piso y techo del túnel de viento limita la curvatura normal que adquiriría el flujo libre que ocurre en cualquier cuerpo que genera sustentación. De acuerdo a esto, un perfil en un túnel de sección cerrada genera más sustentación y momento respecto al cuarto de cuerda para un ángulo de ataque que en condiciones de flujo libre.

Para corregir este efecto, se debe considerar correcciones sobre el ángulo de ataque utilizando la siguiente expresión de Allen y Vincenti: 


$$
\Delta \alpha_{s c}=\frac{57.3 \sigma}{2 \pi}\left(C_{l u}+4 C_{m\left(\frac{c}{4}\right) u}\right)
$$

Donde nuevamente se utilizan variables medidas para las condiciones de ensayo, en este caso, la sustentación $\left(\mathrm{C}_{\mathrm{lu}}\right)$ y el momento de cabeceo respecto al cuarto de cuerda del modelo $\left(\mathrm{C}_{\mathrm{mc} / 4 \mathrm{u}}\right)$.

\section{Anexo II.V: Efectos dinámicos en las cercanías de la pared}

Como fue mencionado anteriormente, no hay una metodología probada y aceptada globalmente para poder realizar correcciones de las mediciones en túnel cuando hay presente efectos dinámicos involucrados, como grandes desprendimientos vorticosos, grandes estelas no estacionarias, elementos rotantes a alta velocidad, etc. Lo más cercano a ello es una tesis de maestría de Ross del 2010, donde muestra un procedimiento de correcciones para medición de aerogeneradores de eje vertical en túnel de viento para distintas relaciones de bloqueo. En dicho trabajo se presentan mediciones de la presión estática en las paredes del túnel de viento para distintas relaciones de bloqueo, mostrando una gran influencia de este parámetro, incluso para valores de bloqueo menores al $5 \%$. Producto de dichos resultados, se procedió a analizar, mediante el rack de presiones mencionado en el desarrollo del trabajo, analizando los sensores que se encuentran en la zona más cercana a las paredes del túnel de viento, y analizar si los efectos dinámicos están presentes. Si los efectos dinámicos son despreciables, indica que el flujo en las cercanías de la pared no se ve afectado por los desprendimientos de flujo, indicando que tiene un comportamiento similar al de las condiciones estacionarias de ensayo. En este caso, la presión analizada, no es la presión estática, sino la presión dinámica, dado que no se tiene información sobre la evolución de la presión estática en dichas condiciones.

En los gráficos a continuación, las señales se detallan en función de la altura adimensional con la cuerda medido desde el eje de rotación del ala, que se encuentra en la zona central de la sección de prueba. Recordando que la sección del túnel posee $40 \mathrm{~cm}$ de alto, y el ala una cuerda de $10 \mathrm{~cm}$, una distancia adimensional (y/c) de 1, representa una distancia de $10 \mathrm{~cm}$. En particular, la toma de presión más alejada se encuentra a una distancia de 1,07 cuerdas hacia arriba del modelo, lo cual representa 10,7 cm, desde el eje de rotación, representando así una distancia a la pared de $9,3 \mathrm{~cm}$. Si bien esta distancia es relativamente grande, si en esta zona no se observan efectos dinámicos en las presiones, podemos asumir que los efectos dinámicos en la pared son despreciables, con lo cual el método de corrección para condiciones estáticas es adecuado.

Desde la Figura II.2 a la Figura II.4 se puede observar la evolución temporal del coeficiente de presión, adimensionalizado con la presión dinámica de referencia, para dos velocidades de corriente libre (4 y $10 \mathrm{~m} / \mathrm{s}$ ), a una frecuencia reducida de 0,015 , para comparar el efecto de la velocidad incidente, y además, se visualizan las señales para la mayor frecuencia reducida $(0,06)$ para analizar si se observa un cambio en el desarrollo de las presiones con el aumento de la frecuencia reducida. Todas las figuras muestran los resultados para las dos condiciones turbulentas analizadas en el trabajo. A partir del análisis de las señales, se puede observar como el comportamiento es muy similar al modificar la velocidad incidente, y la frecuencia reducida del movimiento, sin observar grandes modificaciones en el comportamiento (columna izquierda), lo único que se puede apreciar, es un aumento del apartamiento de las señales, al comparar entre la menor velocidad y la mayor velocidad de ensayo, pero esto puede estar inducido por la menor presión dinámica en la adimensionalización. Un efecto notorio, es un aumento en el desvío respecto de la media, cuando se modifica la turbulencia incidente, lo cual es esperable, producto del aumento de la turbulencia del flujo libre. 

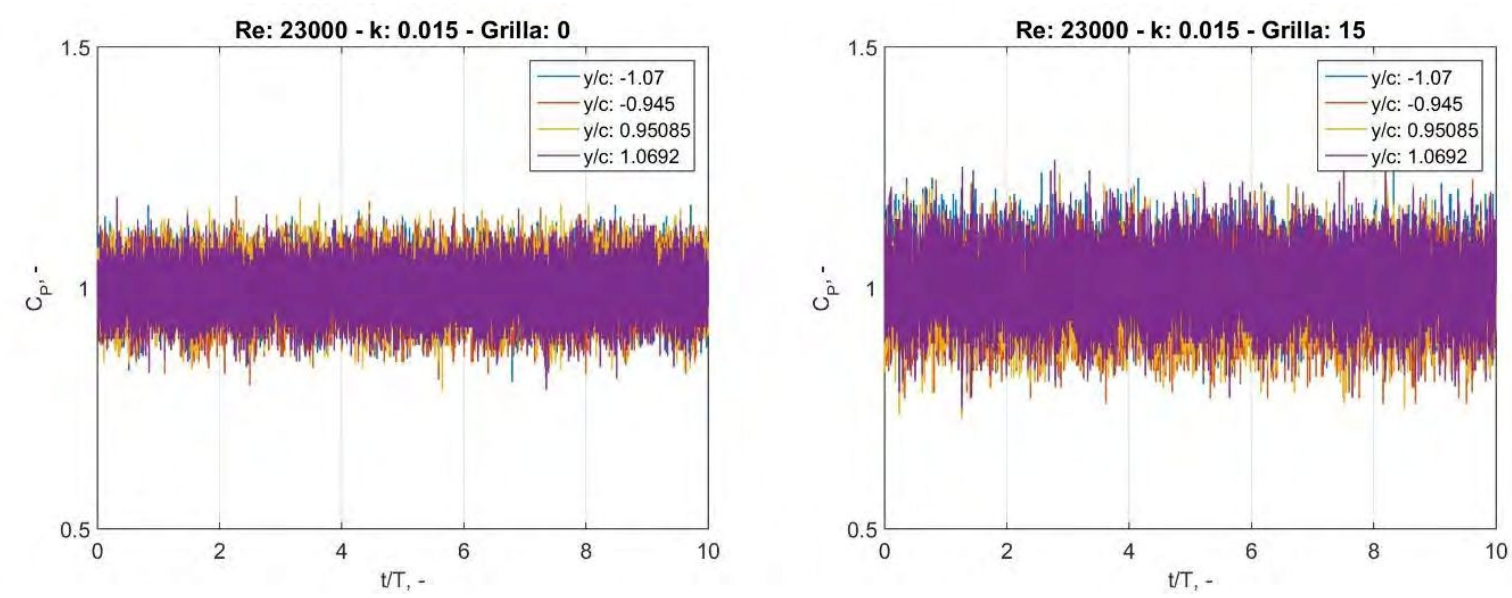

Figura II.2. Evolución temporal del coeficiente de presión para Reynolds 23000, frecuencia reducida 0,015 y ambas grillas turbulentas.
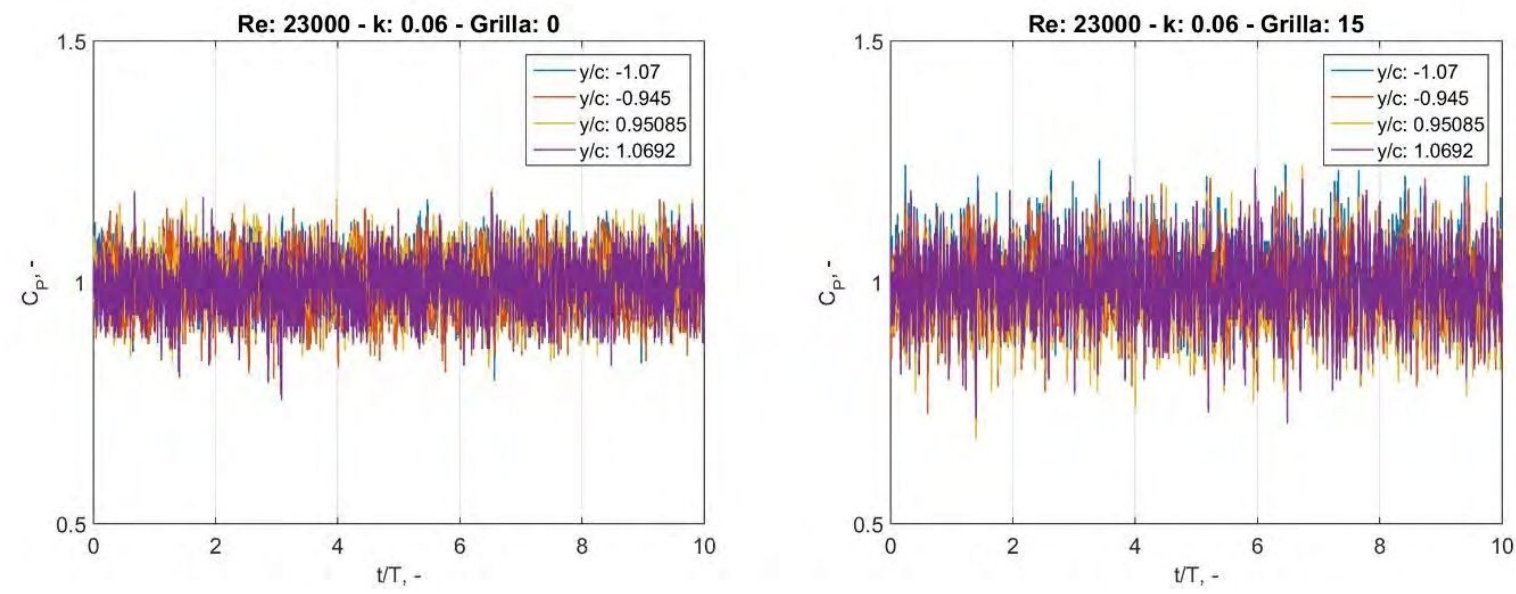

Figura II.3. Evolución temporal del coeficiente de presión para Reynolds 23000, frecuencia reducida 0,06 y ambas grillas turbulentas.
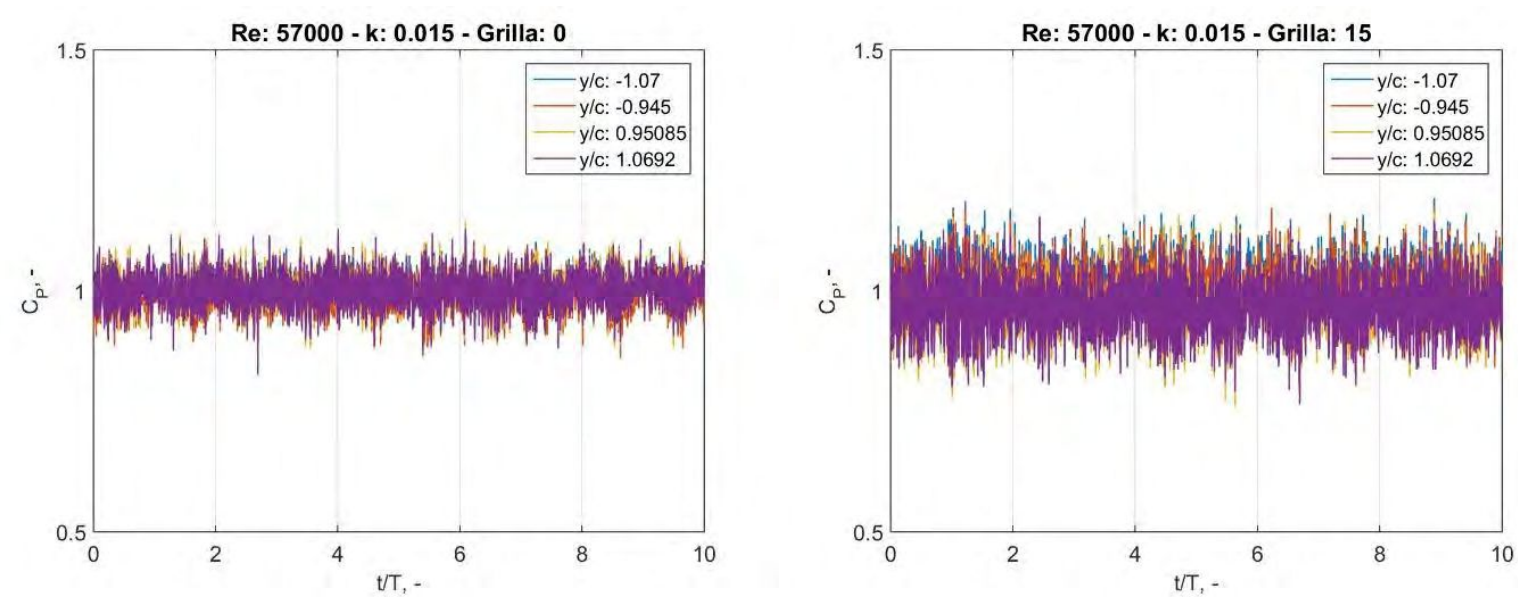

Figura II.4. Evolución temporal del coeficiente de presión para Reynolds 57000, frecuencia reducida 0,015 y ambas grillas turbulentas

Para poder analizar en mayor detalle los desvíos sobre la presión media, se analizó el valor del desvío medio cuadrático (rms, por sus siglas en inglés), para analizar si hay un efecto dominado por la frecuencia del movimiento. Para poder realizar una comparación directa, se graficó la presión media con su valor rms, en función de la frecuencia reducida, para el caso de estudio de menor velocidad de corriente libre (Reynolds 
23000), para ambas condiciones de flujo turbulento. Ver Figura II.5. En ambas figuras se puede observar como el patrón de desvío cuadrático medio, así como la presión media siguen una tendencia muy similar, para ambas condiciones de flujo incidente, en función de la frecuencia reducida, lo cual indica que la velocidad de rotación del modelo no afecta las presiones de forma dinámica en las cercanías de la pared, determinando que es posible realizar las correcciones de las cargas aerodinámicas utilizando el método de corrección en condiciones estáticas.
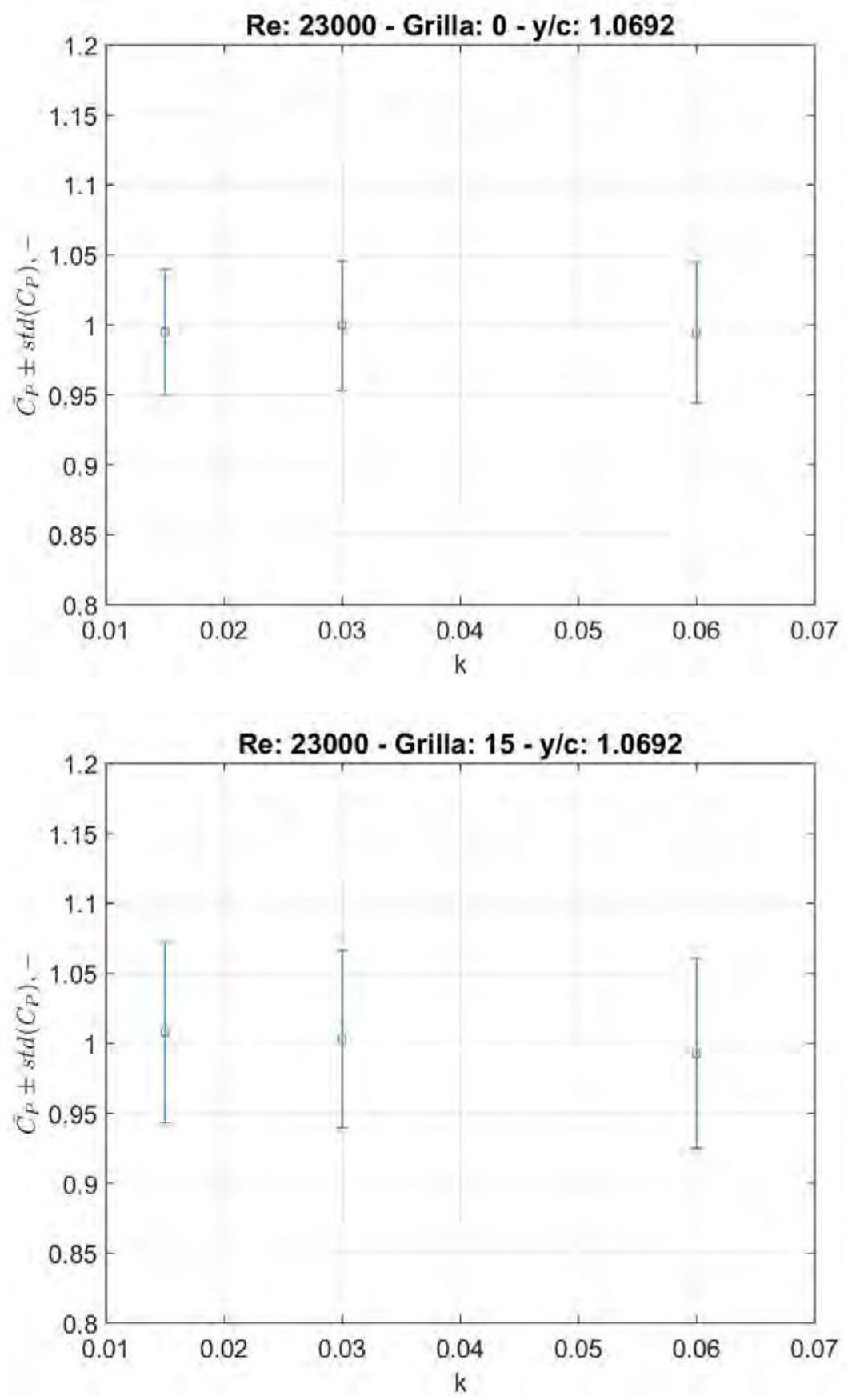

Figura II.5. Valor medio de presión y desvío cuadrático medio en función de la frecuencia reducida, para Reynolds 23000 y ambas condiciones de turbulencia. 


\section{Anexo III: Validación del método de tarado dinámico}

Dado que, al tener un objeto acelerado se producen esfuerzos inerciales que se van a medir en forma simultánea con la fuerza aerodinámica, y para este trabajo es de interés únicamente la fuerza aerodinámica, se probaron distintas formas para medir realizar el tarado de la fuerza inercial en función del movimiento realizado por el ala.

Primeramente, se realizaron mediciones a las distintas velocidades de rotación, pero sin viento incidente. Dicho ensayo se realizó con el objetivo de caracterizar las cargas dinámicas que genera el ala al rotar, más allá de la componente aerodinámica. Como es un ala rotante, esta generará un movimiento de aire conocido como masa aparente. En los cálculos teóricos, es determinada mediante el cálculo de la inercia de un cuerpo circular de diámetro equivalente a una cuerda, por lo que es conocido como fuerza de masa aparente o masa virtual.

$$
F=\frac{\rho c^{2} \pi}{4} \dot{V}
$$

Sin embargo, la velocidad es la velocidad normal al centro del ala, con lo cual dependerá de la ubicación del punto de rotación. En nuestro caso, este punto se encuentra localizado en el centro del ala, siendo nulo el efecto de masa aparente según se demostró en el reporte AVT202.

La Figura III.1 y Figura III.2 muestran la componente axial y normal medidas por la balanza durante las rotaciones a distintas velocidades (se muestran 2 velocidades, correspondientes con frecuencia 0,51 y $1,27 \mathrm{~Hz}$ respectivamente) junto con la misma señal cuando se le aplica el tarado de la balanza obtenido por cargas estáticas sin viento. Es decir, se interpola el valor de carga para cada ángulo instantáneo a partir del peso tarado por la balanza para la condición estática. Al observar estas señales, se puede observar como aparece una carga adicional durante el movimiento del ala, la cual al llegar a la condición estacionaria desaparece, muy similar al comportamiento de la masa aparente. Sin embargo, si en vez de analizar lo que pasa con la fuerza normal y axial, analizamos la componente de sustentación y de resistencia (Figura III.3), podemos observar que es un comportamiento meramente de vibración del ala, dado que la media siempre se encuentra en torno al cero, lo cual se condice con lo planteado en el trabajo AVT202.
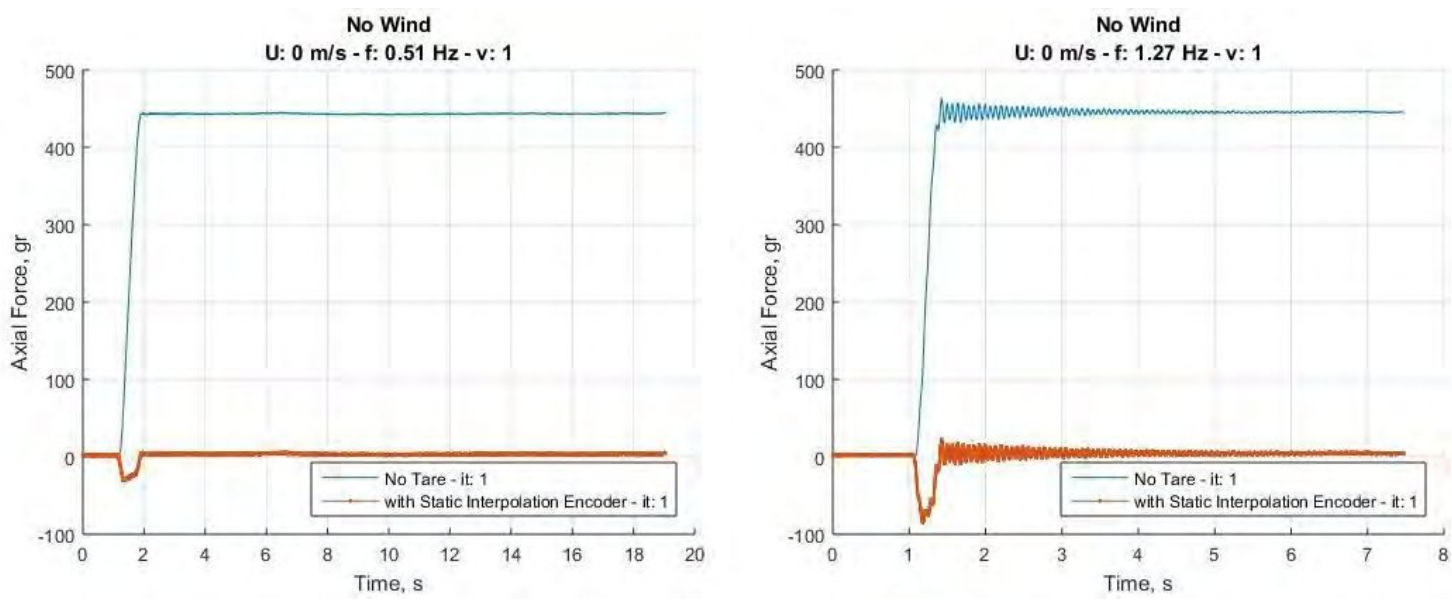

Figura III.1. Componente Axial y Normal de las fuerzas medidas en el caso dinámico sin viento para frecuencia 0,51 Hz. 
“Estudio aerodinámico experimental en flujo turbulento de bajo Reynolds sobre alas con movimiento de cabeceo"
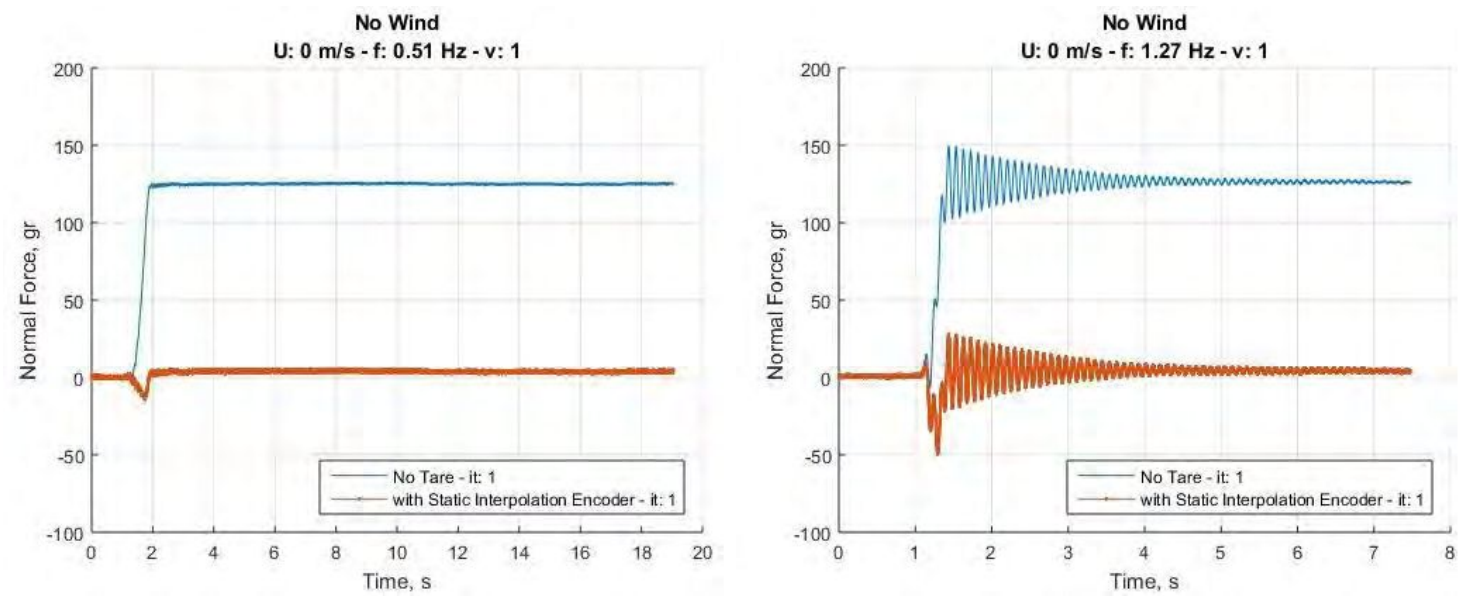

Figura III.2. Componente Axial y Normal de las fuerzas medidas en el caso dinámico sin viento para frecuencia $1,27 \mathrm{~Hz}$.
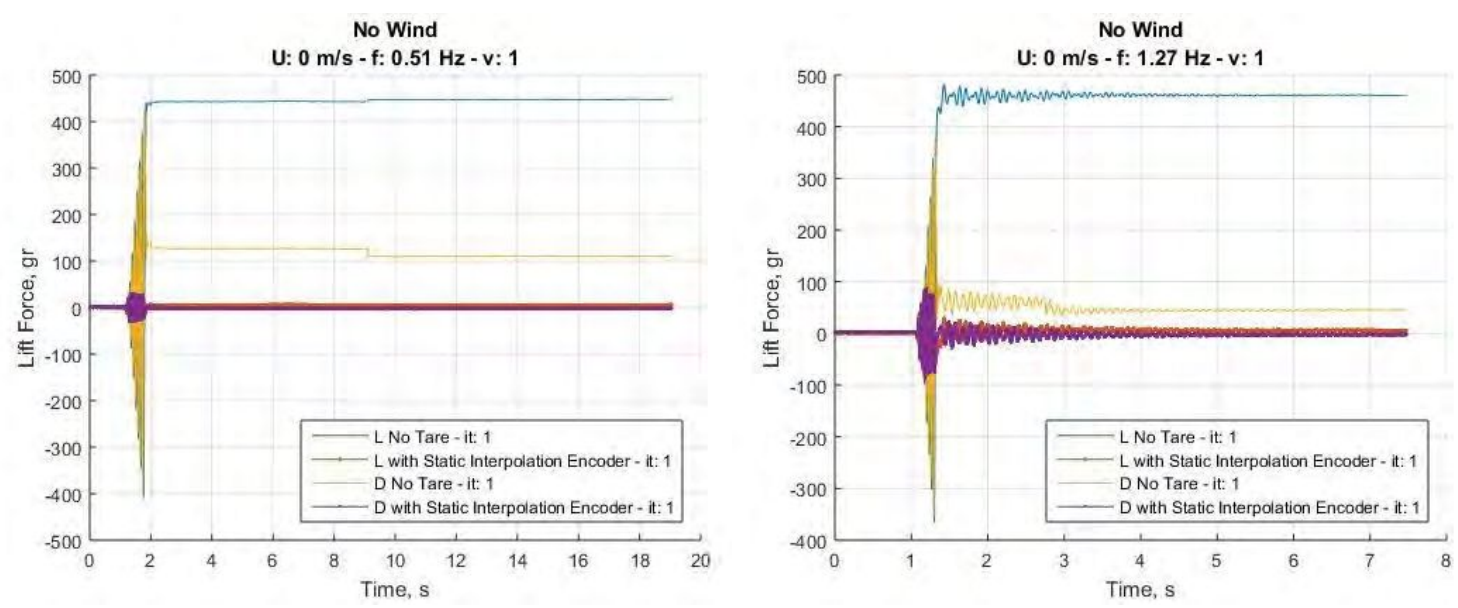

Figura III.3. Comparación entre fuerzas sin tarado y con un tarado de cargas estáticas

A su vez, se realizaron visualizaciones mediante la misma técnica de humo mencionada anteriormente, para verificar si se observa el movimiento de flujo de aire, a pesar de que la velocidad de la corriente libre sea nula. En la Figura III.4 se puede observar una fotografía del campo de flujo, para una frecuencia de oscilación de $0,8 \mathrm{~Hz}$. Con un círculo se demarca la zona de la estela donde se pueden observar claramente la formación de distintas estructuras coherentes, las cuales interactúan entre ellas, formando una calle de Von Kármán invertida, producto de que los vórtices desprendidos desde el borde de fuga se mueven hasta la parte superior de la calle y viceversa.

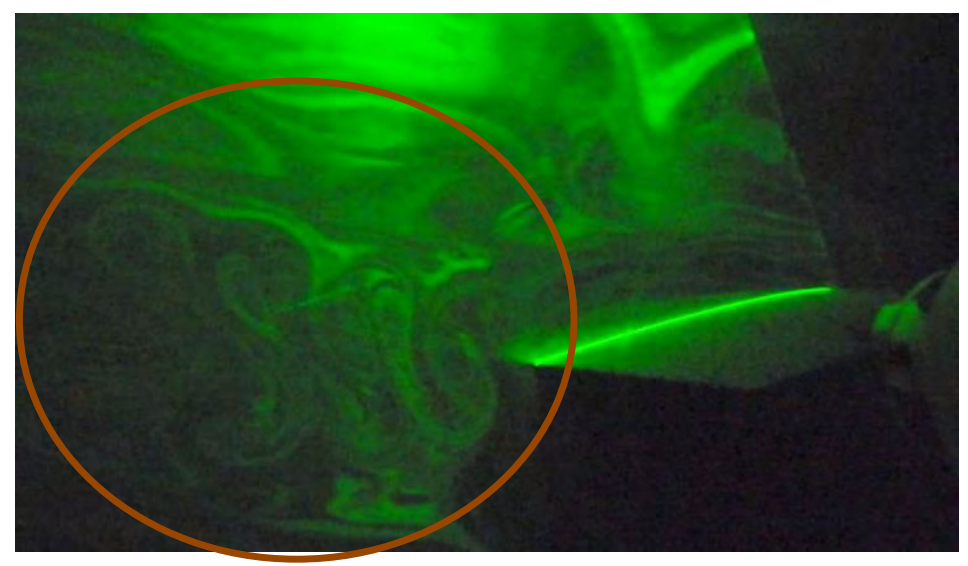

Figura III.4.Visualización en perfil SD8020 sin flujo incidente, frecuencia de oscilación 0,8 Hz. 
Para poder restar el efecto de fuerza inercial durante la rotación del ala, y la generación de los vórtices mostrados en la figura anterior, se realizaron mediciones con un elemento masa equivalente, el cual tiene una masa, posición de cg y momento de inercia respecto al eje de rotación con menos de $0,5 \%$ de diferencia respecto al ala. Este elemento equivalente está compuesto por un conjunto de cilindros, lo cual minimiza la cantidad de aire a mover, donde se destaca un contrapeso principal, alojado en el extremo del ala, el cual permite modificar la ubicación del centro de gravedad (L1) a lo largo del eje de rotación, y dos contrapesos menores, que, en función de su ubicación respecto al eje de rotación (R1), permiten modificar el momento de inercia respecto al eje de rotación. Ver Figura III.5.El diseño de este sistema equivalente se realizó en primera medida mediante una metodología teórica, determinando el peso de cada elemento y su ubicación para poder obtener, mediante una serie de iteraciones, las ubicaciones finales deseadas. Con dichos cálculos, se realizó, mediante el uso de software de dibujo asistido por computadora (Catia v5), una verificación de los valores obtenidos. Con el elemento final, se procedió a realizar los ensayos a las distintas velocidades de rotación utilizadas, en todos los casos sin viento, repitiendo todos los casos cinco veces.

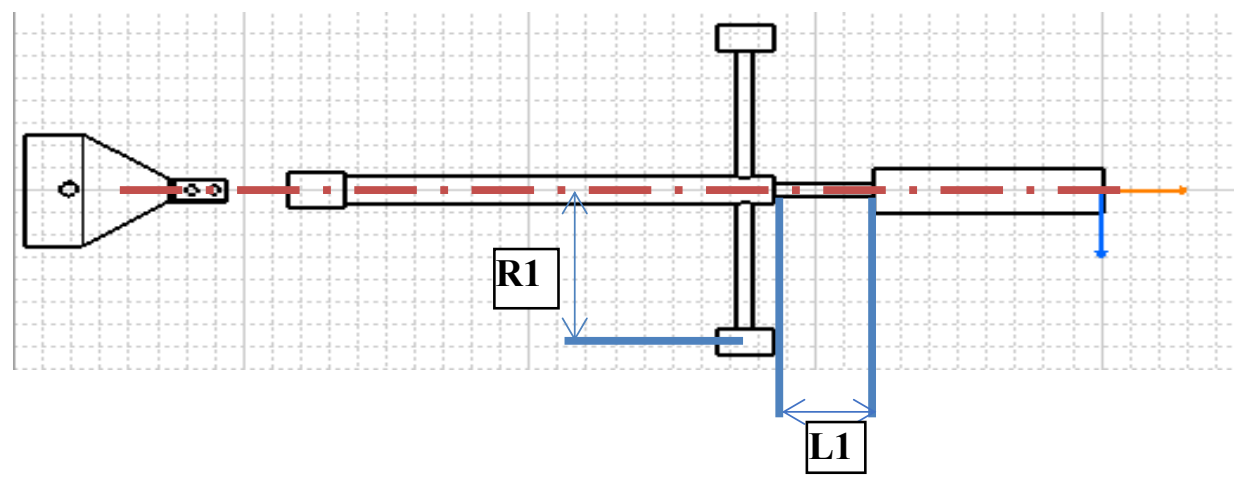

Figura III.5. Esquema de Elemento Equivalente

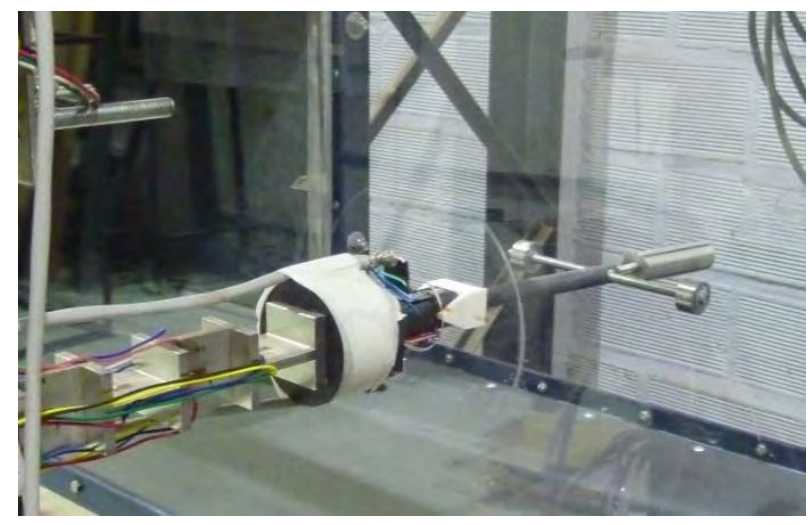

Figura III.6. Montaje del elemento equivalente en el túnel de viento

De esta forma, se verifica si las mediciones obtenidas sin viento son producto del movimiento de una masa de aire (masa aparente) o si es solo un problema de tarado dinámico. En primera instancia se verificó que la cinemática coincida con respecto a la realizada por el ala, lo cual se puede observar en la Figura III.7.

Con la cinemática verificada, se procedió a analizar las fuerzas sensadas para el elemento equivalente, así como para el modelo alar sin flujo incidente. Para ello en primera instancia se graficaron ambas señales sensadas (Figura III.8), con el ala sin flujo ("Wing no tare") y el ala con el método de tarado estático, mencionado anteriormente ("Wing with Static tare"), y las mismas condiciones para el elemento equivalente, primero sin tarado alguno ("Eq No tare") y el elemento equivalente con su tarado estático ("Eq with Static tare"). Allí se puede observar como, a pesar de realizar un tarado con el valor del peso de cada elemento en función del ángulo del modelo en cada instante, durante el movimiento se producen esfuerzos inerciales. A su vez, comparando la 


\section{“Estudio aerodinámico experimental en flujo turbulento de bajo Reynolds sobre alas con movimiento de cabeceo"}

amplitud de oscilación entre el modelo alar y el elemento equivalente, se puede observar como para el modelo alar la amplitud es mayor, lo que indica que hay otras fuerzas involucradas (fuerzas aerodinámicas). Producto de estos resultados, se decidió para todo el trabajo de la tesis, implementar el método de tarado dinámico mediante el elemento equivalente, para poder obtener la medición de fuerza aerodinámica, descontando los esfuerzos inerciales. Cabe aclarar que el elemento equivalente fue reconfigurado para cada modelo alar, producto que al ser de materiales distintos, aluminio la placa plana y fibra de vidrio el perfil SD8020, estos poseían ligeras diferencias en los pesos y momentos de inercia, con lo cual el elemento equivalente tiene una configuración diferente para cada modelo alar.
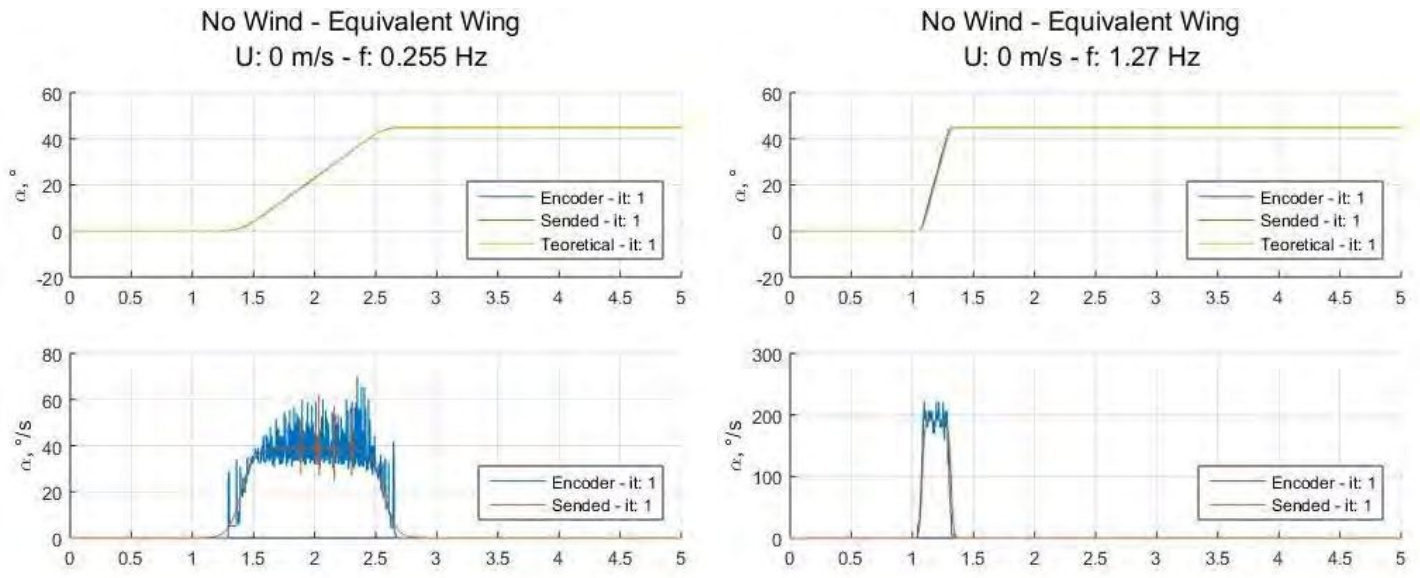

Figura III.7. Comparación de movimiento realizado por el elemento equivalente y el enviado por el software para dos
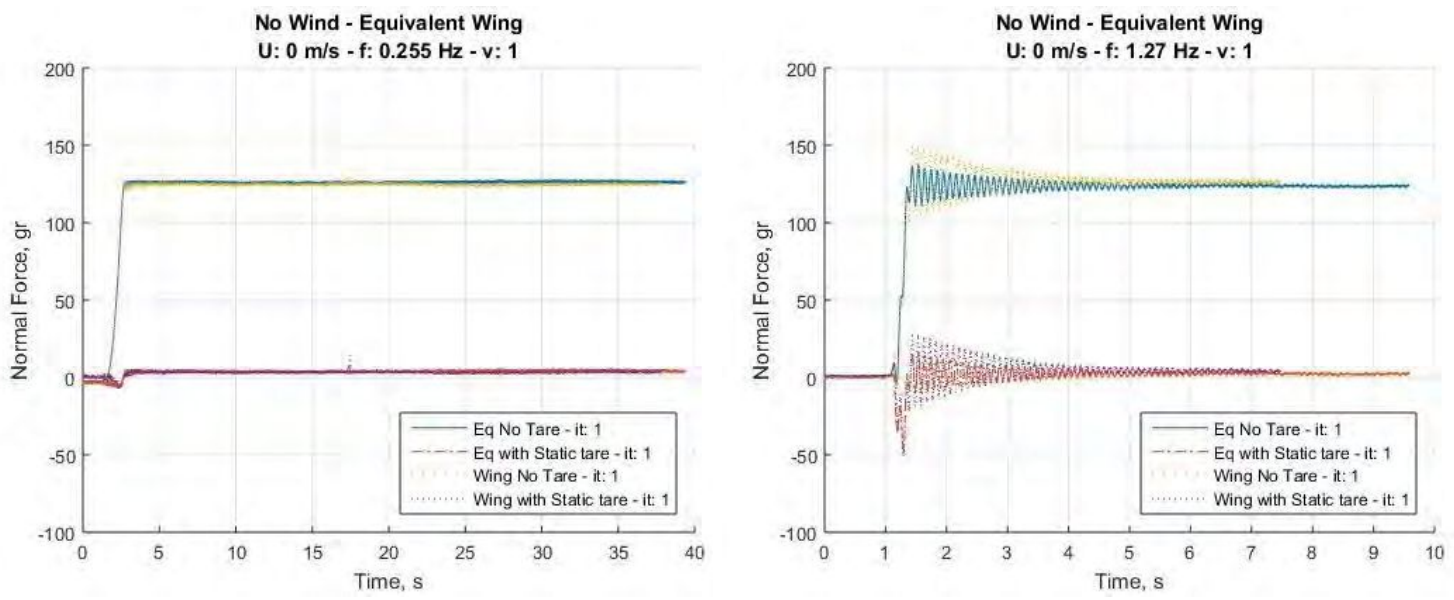

Figura III.8. Comparación directa de las fuerzas con ala y elemento equivalente sin viento. 


\section{Anexo IV: Análisis de repetitividad de cargas aerodinámicas}

Uno de los puntos más importantes a la hora de realizar mediciones experimentales, según las buenas prácticas, es asegurar la repetitividad de los ensayos, así como la determinación de los errores de medición. En este anexo abordaremos dicha temática. En primera instancia, producto de la metodología propuesta, así como las características del movimiento utilizado, era de suma importancia asegurar que todas las iteraciones realizadas del ensayo, aseguraran el mismo movimiento, o con un error acotado, y, por sobre todas las cosas, que el movimiento sea el mismo cuando se realizaron las mediciones con el elemento equivalente. Si bien este análisis se realizó para todas las condiciones de ensayo, por practicidad y facilidad de lectura solo se mostrarán algunos de los resultados generados, que sean los más representativos.

En la Figura IV.1 se puede observar la evolución temporal del ángulo de ataque de cinco iteraciones del modelo de placa plana, junto con su elemento equivalente, para la condición de Reynolds 23.000, aunque resultados muy similares han sido encontrados en el resto de los ensayos. Allí se puede observar diferencias en el ángulo final del ángulo de ataque, que no superan el valor de $0,5^{\circ}$ (3\% del ángulo máximo), mientras que para la carrera ascendente y descendente se observa la superposición de las curvas, indicando una muy buena repetitividad en dicha zona. Luego, se realizó el mismo análisis, comparando los distintos ciclos, dentro de cada iteración, ver Figura IV.2. Allí se puede observar como el movimiento de cada ciclo, dentro de una medición es muy buena para todas las frecuencias reducidas ensayadas, presentándose un pequeño desfasaje para el caso de mayor frecuencia reducida, donde el desvío máximo en dicha condición es de $2 \%$ del periodo del movimiento.

Con el movimiento del modelo verificado, asegurando la repetitividad del mismo, se procedió a verificar que el valor medio de las cargas mensuradas sea un valor adecuado, sin presentarse grandes apartamientos del mismo. Para validar dicha situación, se procedió a graficar la evolución de las cargas en el tiempo adimensionalizado con el periodo del movimiento, para distintas iteraciones, y en distintas condiciones de ensayo. En la Figura IV.3 se puede observar la evolución del coeficiente de sustentación para el número de Reynolds 23.000, en ambas condiciones de flujo turbulento, y las tres frecuencias reducidas. Se muestran los resultados en estas condiciones dado que, al ser la condición de ensayo con menor velocidad incidente, por ende menor presión dinámica, las fuerzas son menores, siendo crítica la condición de medición mediante balanza aerodinámica. En esta figura se puede observar como el comportamiento medio de todas las señales se superpone, encontrando algunas variaciones, en la zona de ángulo constante $(0,45<\mathrm{t} / \mathrm{T}<0,55)$, lo cual puede estar asociado a las pequeñas diferencias en el ángulo final alcanzado por el movimiento. Para finalizar, se analizó la repetitividad de la evolución del coeficiente de sustentación, pero respecto al ángulo de ataque, observando resultados muy similares a los mencionados para el análisis respecto al tiempo adimensional con el periodo del movimiento. Ver Figura IV.4.

Con el análisis realizado, se aseguró que los resultados presentados en el desarrollo de este trabajo representan valores que pueden ser reproducibles a lo largo del tiempo, asegurando que las condiciones de ensayo sean las mismas que las utilizadas. 
"Estudio aerodinámico experimental en flujo turbulento de bajo Reynolds sobre alas con movimiento de cabeceo"
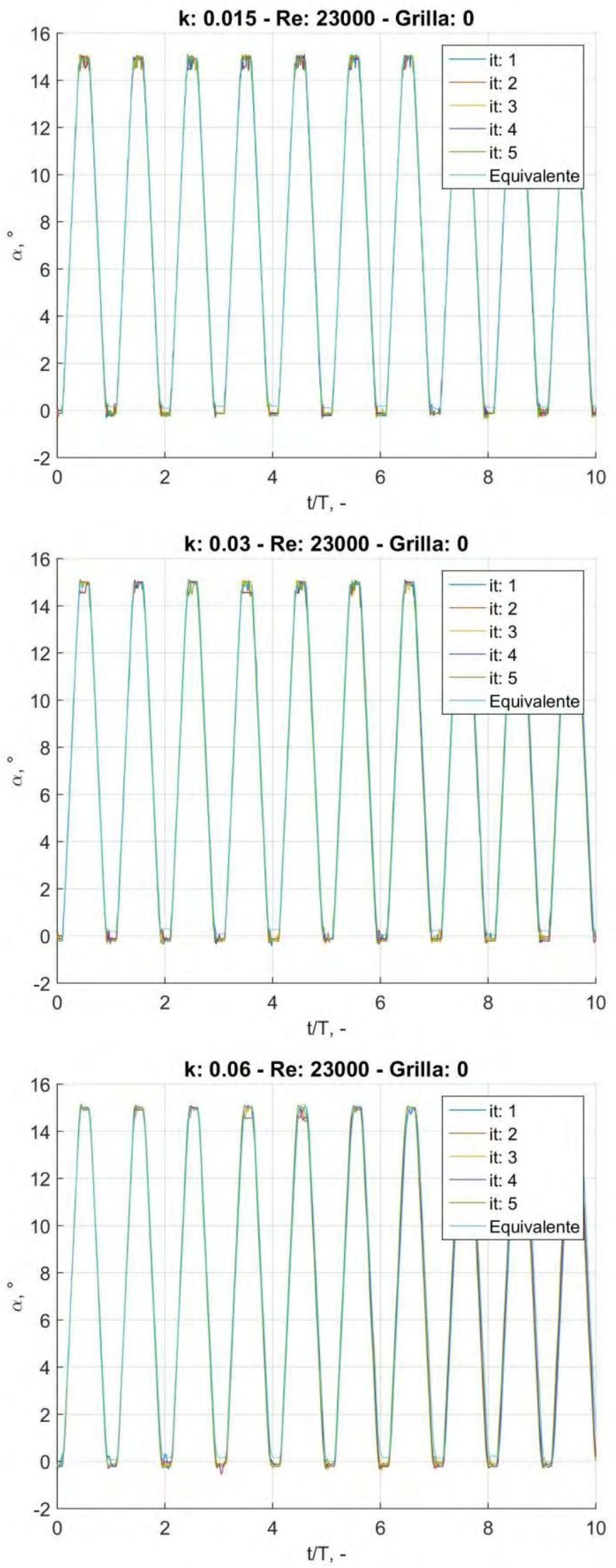

Figura IV.1. Verificación del movimiento para tres frecuencias reducidas distintas, para modelo alar y elemento equivalente. 

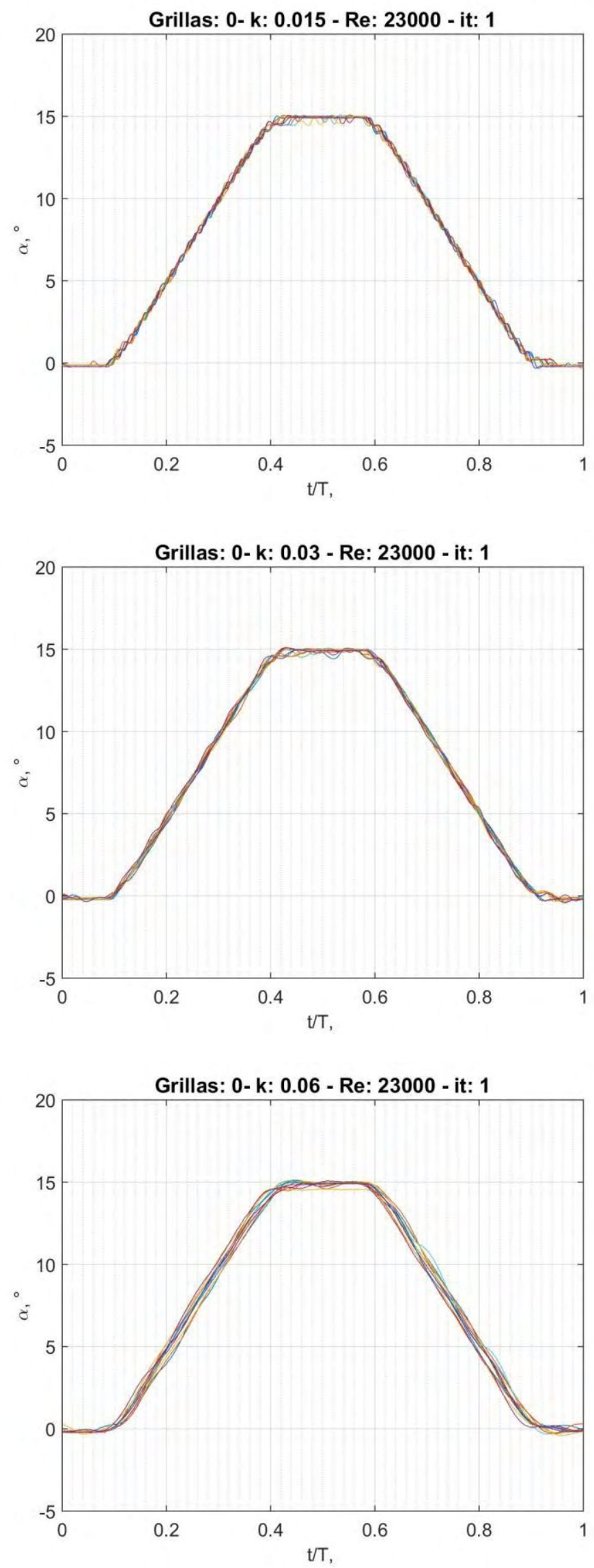

Figura IV.2. Comparación de la cinemática del modelo alar para los distintos ciclos en una iteración, para cada frecuencia reducida ensayada. 
“Estudio aerodinámico experimental en flujo turbulento de bajo Reynolds sobre alas con movimiento de cabeceo"
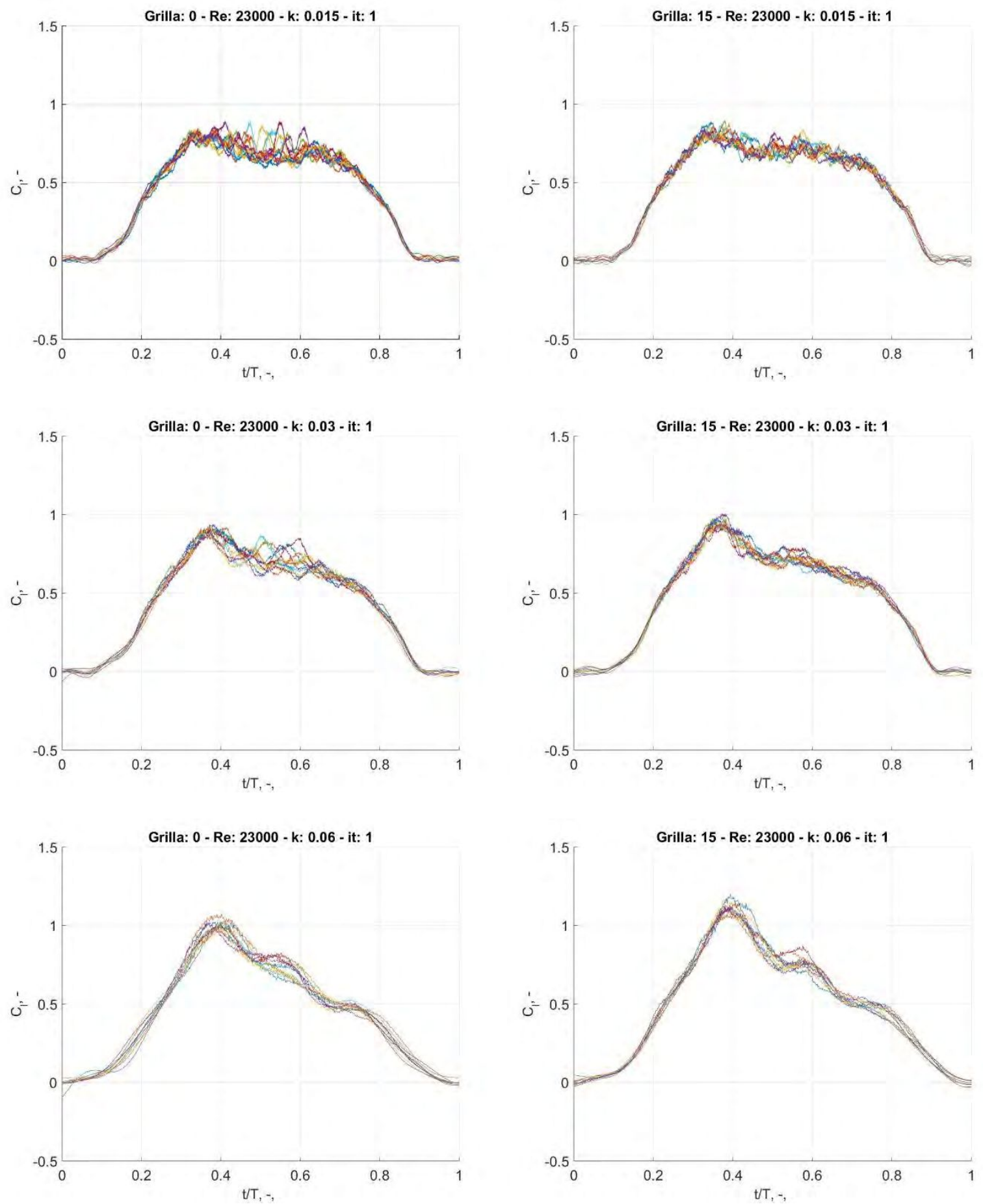

Figura IV.3. Coeficiente de sustentación de los 10 ciclos para tres frecuencias reducidas con número de Reynolds 23.000 y ambas condiciones de turbulencia. 

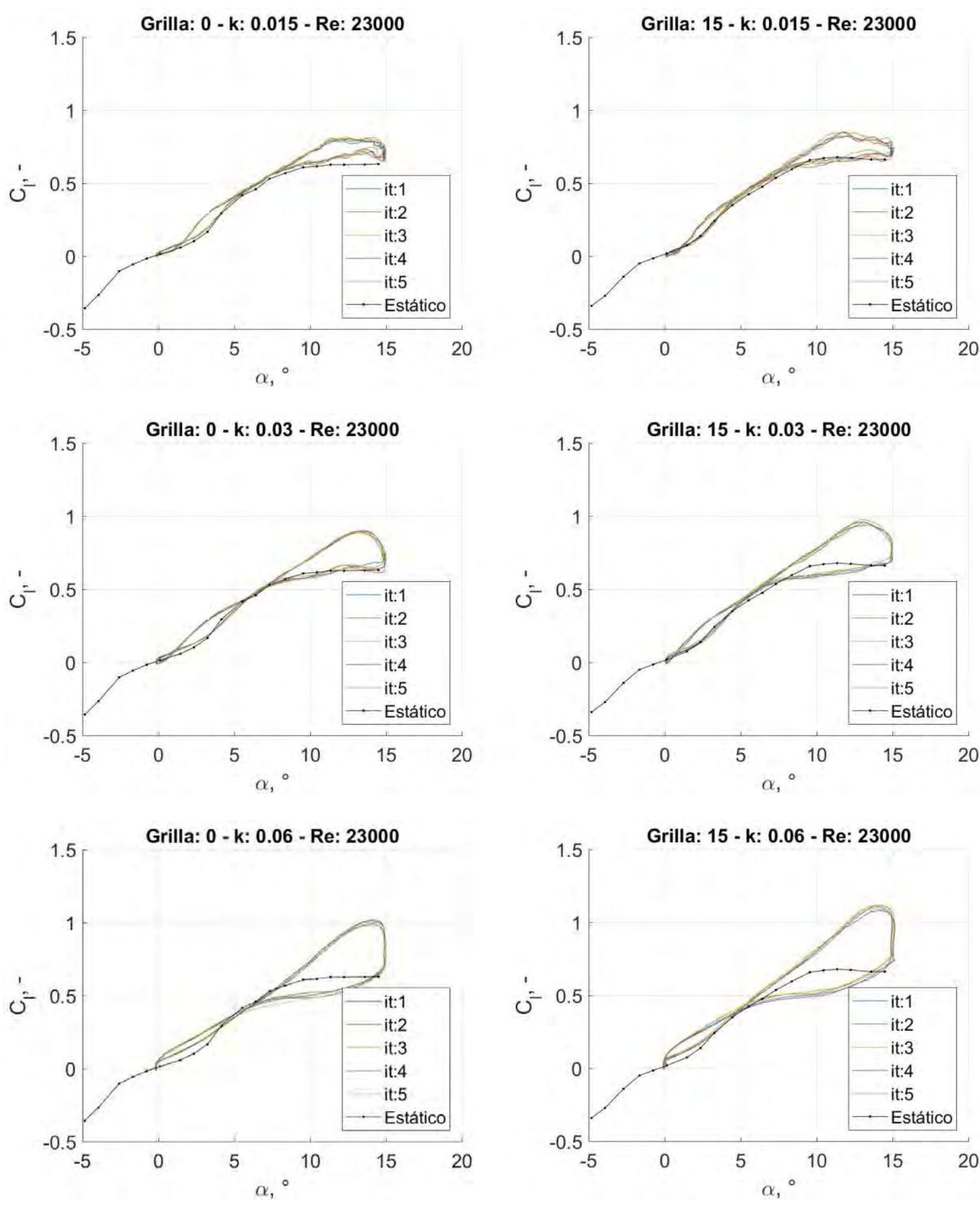

Figura IV.4. Comparación del coeficiente de sustentación para distintas iteraciones del ensayo. Reynolds 23.000, tres frecuencias reducidas y ambas condiciones de turbulencia 


\section{Anexo V: Validación de mediciones mediante Rack de Presiones}

En este apartado se resumen los resultados hallados al realizar las mediciones de presiones con rack de presiones a una distancia de una cuerda aguas abajo y comparado con resultados experimentales planteados por distintos autores para la validación del sistema de medición. Como referencia se utilizaron las mediciones experimentales de los coeficientes de sustentación y resistencia en Selig 1995, las cuales fueron realizadas para condiciones de flujo laminar (Figura V.1 y Figura V.2), así como valores obtenidos mediante simulación numérica de los coeficientes de sustentación y resistencia mediante el software XFoil, incorporando la intensidad de turbulencia de 0,5 en las simulaciones para obtener resultados más cercanos a las condiciones de ensayo.

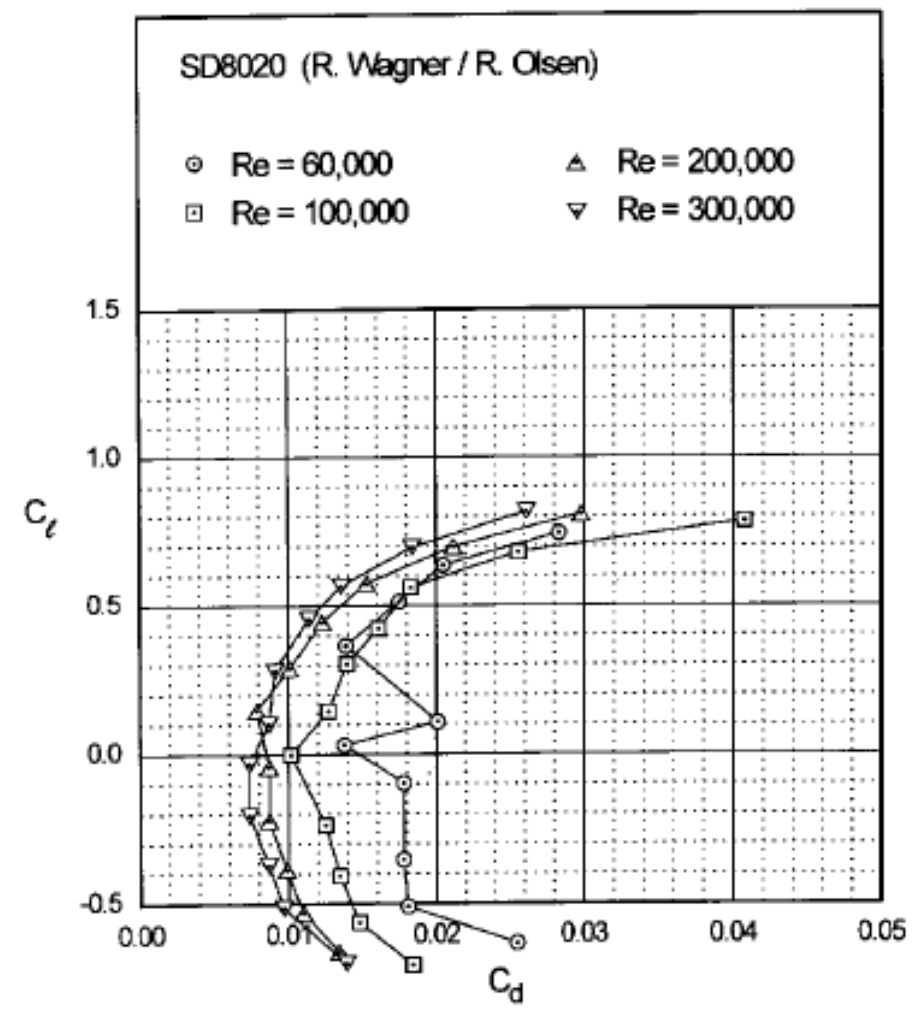

Figura V.1. Polar aerodinámica para distintos Reynolds. Ref.: Selig 1995 
"Validación de mediciones mediante Rack de Presiones"
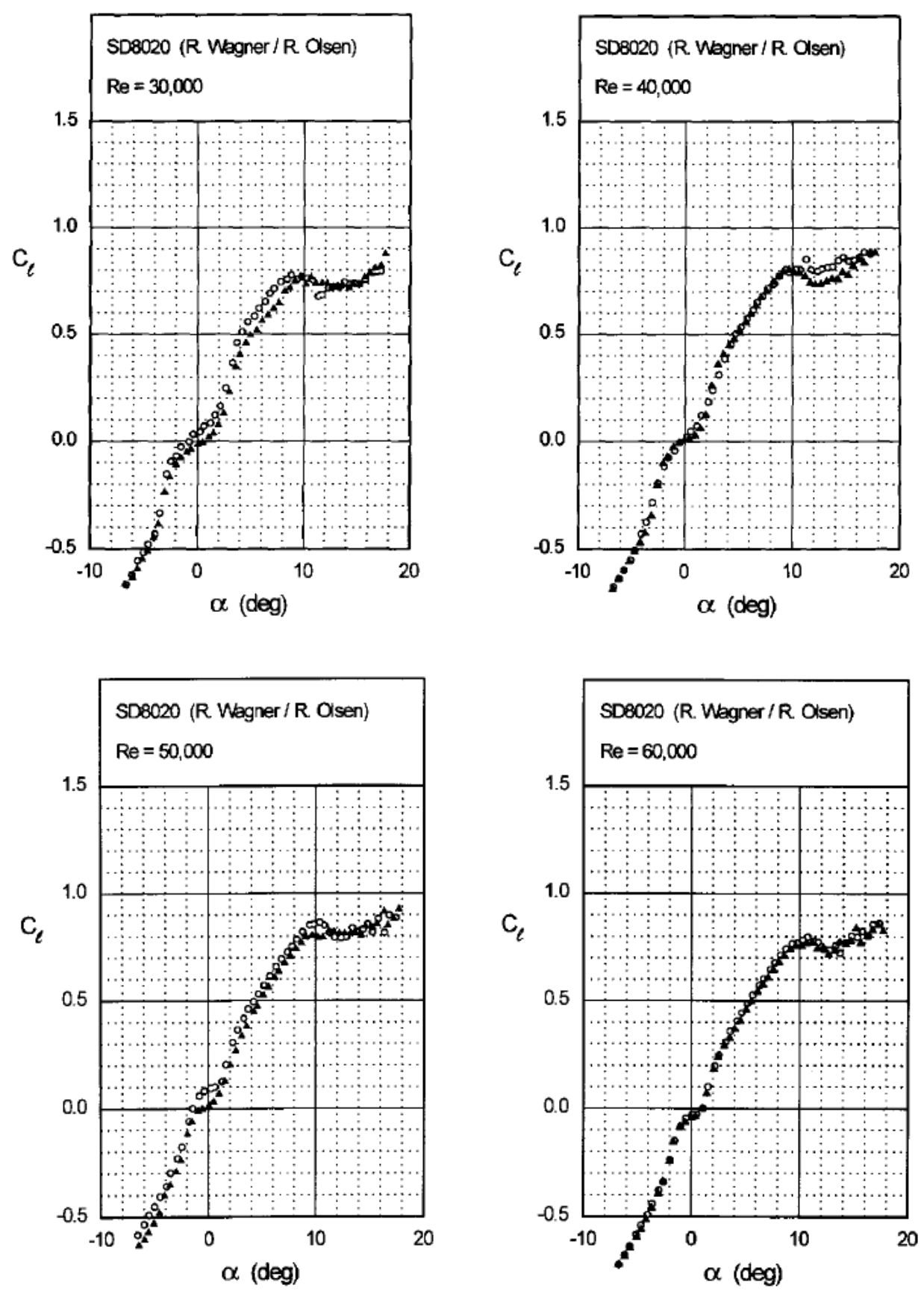

Figura V.2. Coeficiente de sustentación para distintos números de Reynolds. Ref.: Selig 1995 


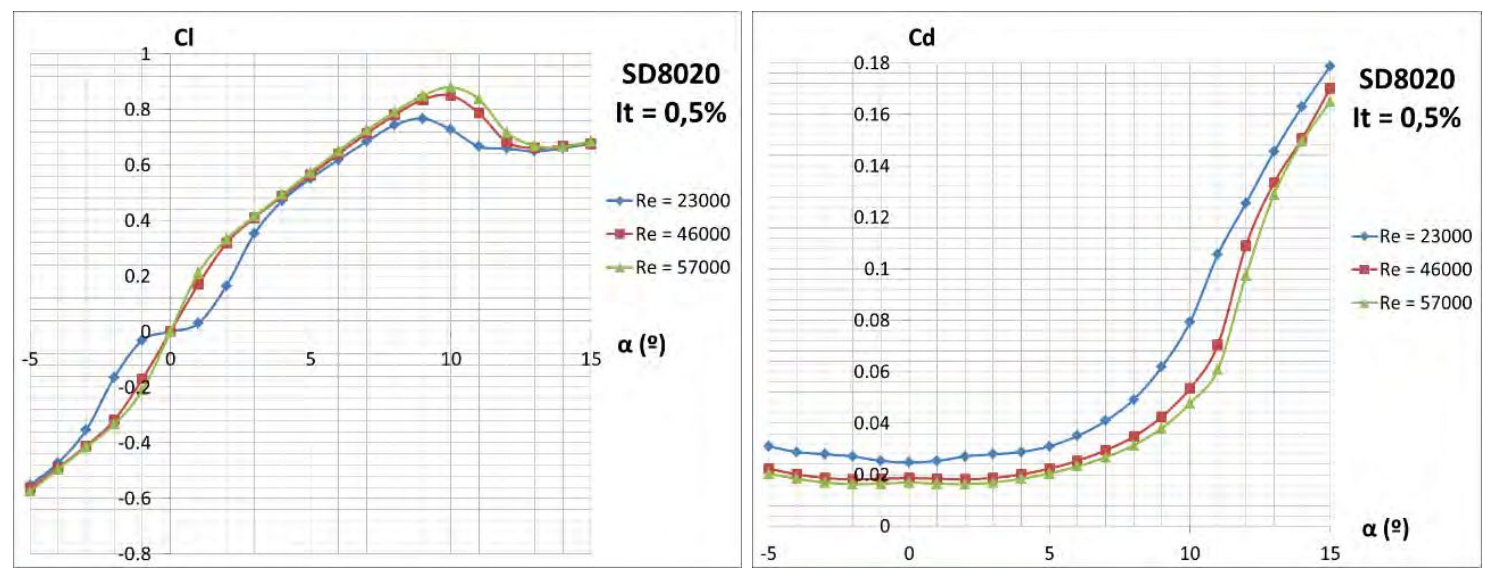

Figura V.3. Coeficientes aerodinámicos simulados con XFoil para la condición de $0,5 \%$ en intensidad de turbulencia.

\section{Anexo V.I: Datos destacados}

A partir de la información obtenida de ensayos experimentales de otros autores y de las simulaciones numéricas se puede observar como el ángulo de entrada en pérdida del perfil es cercano a $9^{\circ} \sim 11^{\circ}$, dependiendo del número de Reynolds, dado que este ángulo se incrementa a medida que aumenta el número de Reynolds, desde 30.000 hasta 60.000 . El valor de resistencia para sustentación nula $\left(0^{\circ}\right.$ de ángulo de ataque, dado que es un perfil simétrico), es de 0,018 en Reynolds 60.000 y 0,02 para Reynolds de 40.000. El coeficiente de máxima sustentación es 0,9 aproximadamente.

\section{Anexo V.II: Distribución de presiones}

En primera instancia se analizan los valores medios de la distribución de presiones en la estela para distintos ángulos de ataque. Los valores medios representan a la media de tres ensayos de 10 segundos cada uno. Se muestran los resultados para cada valor de turbulencia, para tener de referencia su influencia sobre la estela.

Los valores que se muestran en la Figura V.4 se encuentran adimensionalizados con la presión dinámica de la corriente libre, en la estación de medición. Es decir, se consideró el valor medio de las 5 tomas de presión más alejadas en la sección superior del rack de presiones. Se muestran los resultados para ángulo de ataque entre 0 y $15^{\circ}$, cada dos grados de diferencia. Se puede notar como no hay diferencias apreciables en la distribución de presiones, salvo para los ángulos de $7^{\circ}$ y $9^{\circ}$, lo cual puede ser producto de la cercanía al ángulo de ataque de entrada en pérdida, lo cual, la turbulencia genera modificaciones en el punto de transición en la capa límite, produciendo cambios en el campo de velocidades, y por consiguiente de presiones, en la estela. Comparando las gráficas entre $9^{\circ}$ y $11^{\circ}$ se puede observar un incremento en la pérdida de cantidad de movimiento, lo cual involucra una estela mayor, indicando la entrada en pérdida del perfil, como era esperable a partir de los resultados mostrados anteriormente. Otro punto a denotar, es que aparentemente para $9^{\circ}$ en el caso de mayor intensidad de turbulencia, el perfil no muestra signos de desprendimiento total. 

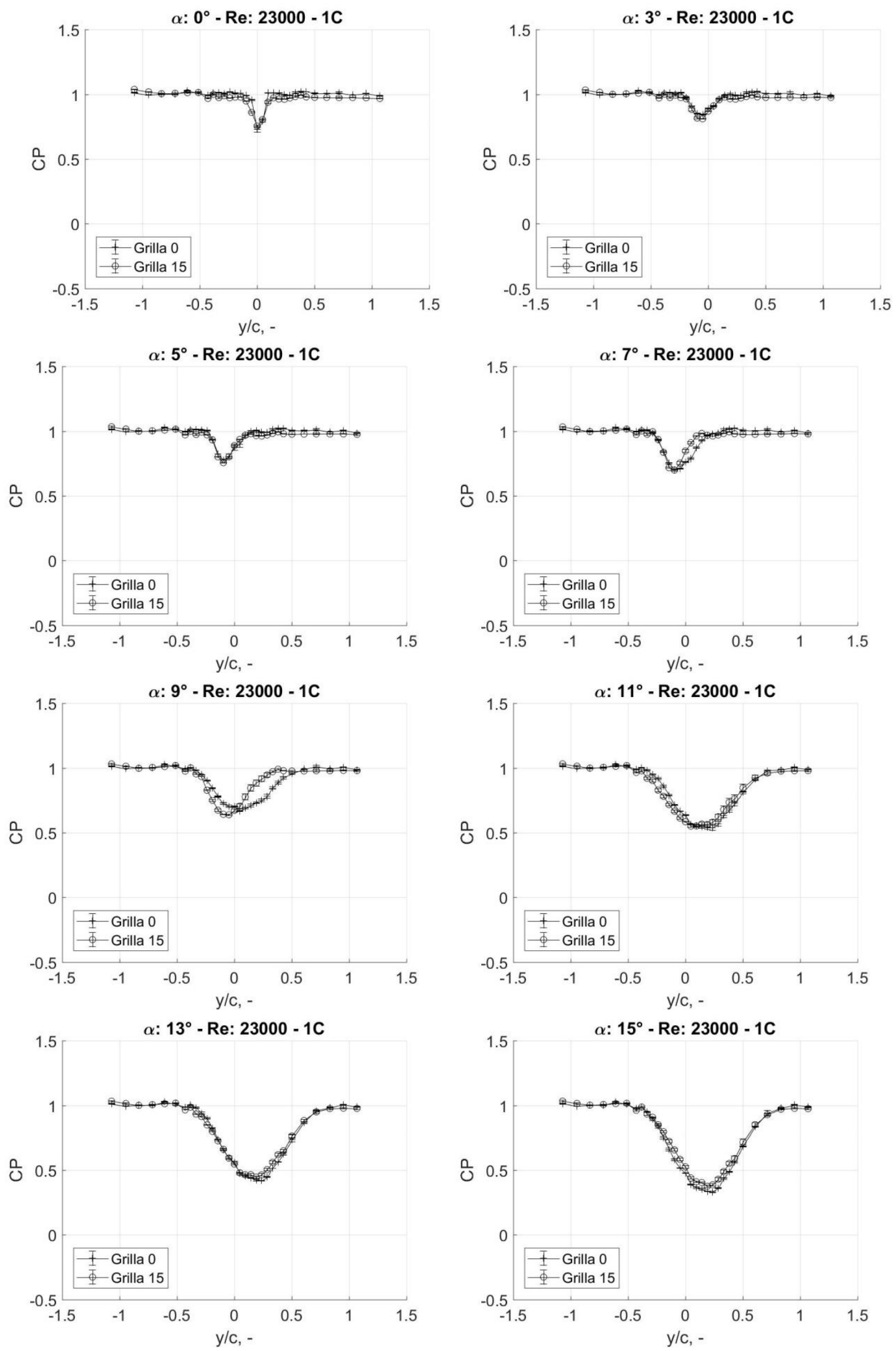

Figura V.4. Distribución de presiones adimensionalizado con la presión dinámica de la corriente libre. 


\section{"Estudio aerodinámico experimental en flujo turbulento de bajo Reynolds sobre alas con movimiento de cabeceo"}

\section{Anexo V.III: Coeficiente de resistencia}

A partir de la distribución de presiones se calcula la cantidad de movimiento y se lo integra para obtener el valor del coeficiente de resistencia. Además, se grafica en forma de referencia el resultado hallado mediante la simulación de XFoil. En la Figura V.5 se puede observar como a pequeños ángulos, el coeficiente de resistencia para el caso de grilla 0 (intensidad de turbulencia $0,5 \%$, coincide con la simulación, aunque dichos resultados no presentan una variación tan notoria respecto al cambio de turbulencia. Al incrementarse el ángulo de ataque los resultados experimentales muestran un valor mayor de resistencia. Dicha diferencia puede ser producto del método de corrección, que no está considerando grandes desprendimientos de flujo, con lo cual, puede que no sea del todo correcta la corrección realizada.
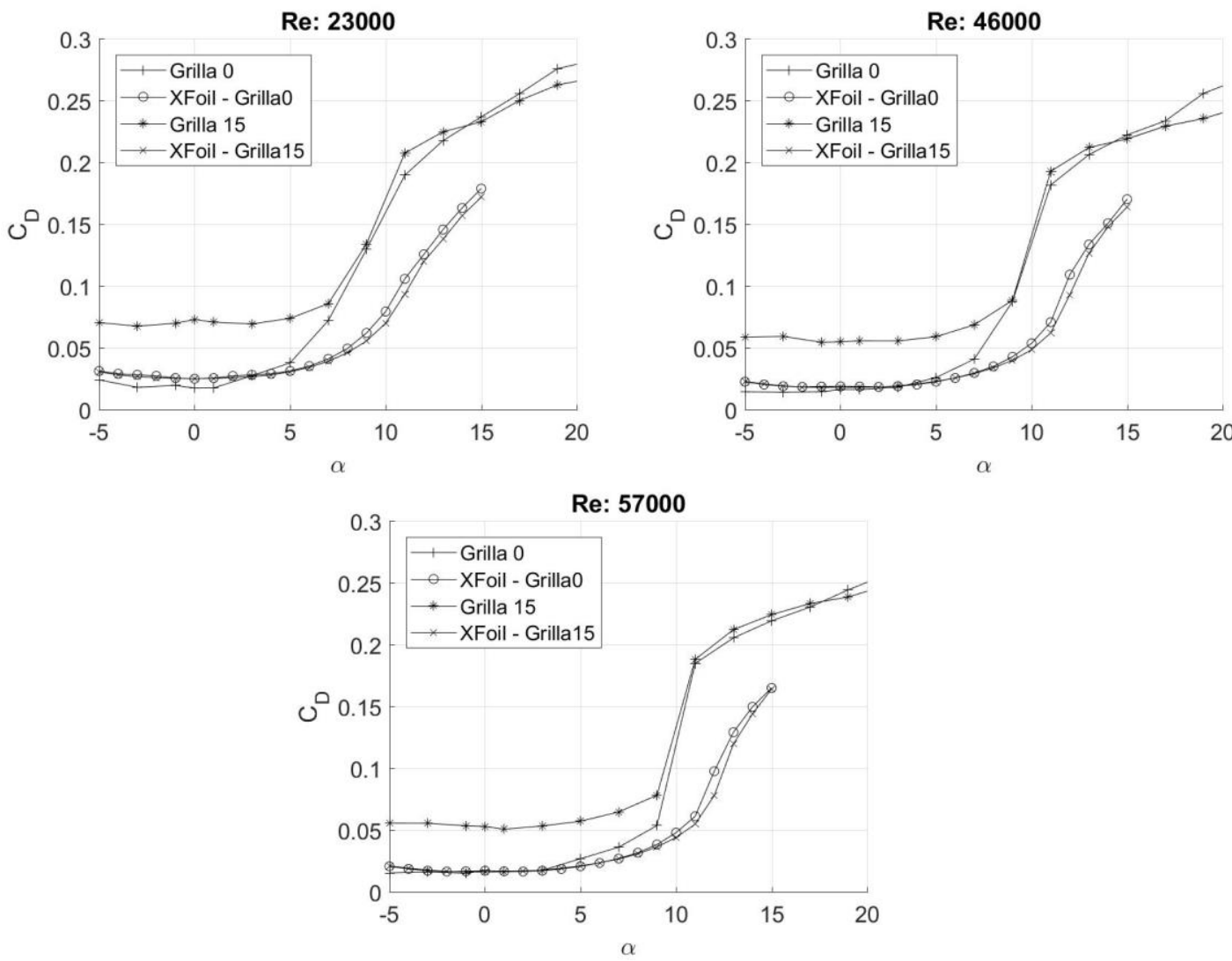

Figura V.5.. Coeficiente de resistencia para distintos números de Reynolds. 


\section{CAPÍTULO 5: Bibliografía}

Aramburu Orihuela, A., Morales, J., Algozino, S., Marañón Di Leo, J. “Influencia de la turbulencia en las características aerodinámicas de perfiles de bajo Reynolds". Cuarto Congreso de Ingeniería Aeronáutica, Córdoba, Argentina, 2016.

Babinsky, H., Stevens, P.R.R.J., Jones, A.R., Bernal, L.P., Ol, M.V. "Low Order Modelling of Lift Forces for Unsteady Pitching and Surging Wings". 54th AIAA Aerospace Sciences Meeting, AIAA SciTech Forum. AIAA 2016-0290

Barlow J., Rae W. and Pope, A. "Low Speed Wind Tunnel Testing”. $3^{\text {rd }}$ Edition. 1999. John Willey \& Son.

Brown, R. H. J., "The Flight of Birds-The Flapping Cycle of the Pigeon," Journal of Experimental Biology, Vol. 25, No. 4, 1948, pp. 322-333.

Carr. "Progress in Analysis and Prediction of Dynamic Stall". Journal of Aircraft. Vol 25. N 1. 1987

Drela. "XFOIL: An Analysis and Design System for Low Reynolds Number Airfoils". Low Reynolds Number Aerodynamics. Springer-Verlag Lec. Notes in Eng. 54. 1989

Drela and Giles "Viscous Inviscid Analysis of Transonic and Low Reynolds number Airfoils". AIAA Journal 1987

Eldredge, J.D., Wang, C.J., and Ol, M. "A Computational Study of a Canonical Pitch-Up", Pitch-down Wing Maneuver". AIAA 2009-3687.

Ellington C. P. 1997 "The aerodynamics of hovering insect flight. III. Kinematics" 305 Phil. Trans. R. Soc. Lond. B. 457

Farge M. 1992. Wavelet transforms and their application to turbulence. Annu. Rev. Fluid Mech. 24:395-

Farge M, Schneider K. 2006. Wavelets: application to turbulence. In Encyclopedia of Mathematical Physics, ed. JP Francoise, GL Naber, TS Tsun, pp. 408-20. Oxford: Academi

Grandlund, K., Ol, M. and Bernal, L. "Experiments on Pitching Plates: Force and Flowfield Measurements at Low Reynolds Numbers". 49 ${ }^{\text {th }}$ AIAA Aerospace Sciences Meetings. AIAA 2011.-672

Jadhav, G., Massey, K., 2007. The development of a miniature flexible flapping wing mechanism for use in a robotic air vehicle. AIAA Paper 2007-668.

Krause L. "Effects of pressure-rake design parameters on static-pressure measurement for rakes used in subsonic free jets". NACA TN 2520. 1951

Leishman JG. Principles of helicopter Aerodynamics. Cambridge University Press, London. 2010.

Lin S H., Hsiao F.Y., Chen C.L. and Shen J.F. "Altitude Control of Flapping-wing MAV Using VisionBased Navigation”. American Control Conference, June 2010, Baltimore, U.S.A.

Marino. "Unsteady Pressure Sensing on a MAV Wing for Control Inputs in Turbulence". PhD Thesis. RMIT University. 2013

McArthur J. "Aerodynamics of wings at low Reynolds Number". 2007. University of Southern California. $\mathrm{PhD}$ Thesis

McCroskey WJ. "The phenomenon of Dynamic Stall”. NASA TM 81264. 1981

Mueller, D., Bruck, H.A., Gupta, S.K., 2010. Measurement of thrust and lift forces associated with drag of compliant flapping wing for micro air vehicles using a new test stand design. Experimental Mechanics 50 (6), $725-735$. 


\section{"Bibliografía"}

Ol M., Bernal L, Kang C.y Shy W. "Shallow and deep dynamic stall for flapping low Reynolds number". Animal Locomotion, pp 321-329. Springer. 2010

Ol M. and Babinsky H. "Unsteady Flat Plates: a Cursory Review of AVT-202 Research". 54th AIAA Aerospace Sciences Meeting, AIAA SciTech Forum. AIAA 2016-285

O'Meara M.M. y Mueller T.J. "Laminar Separation Bubble Characteristics on an Airfoil at Low Reynolds Numbers". AIAA Journal. Vol 20. No 8. 1987.

Ross I. J. "Wind Tunnel Blockage corrections: An application to vertical axis wind turbines". Master Thesis. University of Dayton. 2010.

Schneider K, and Vasilyev O. "Wavelet methods in computational fluid dynamics". Annual Review of Fluid Mechanics, Annual Reviews, 2010, 42, pp.473-503

Selig, M. S. (Ed.). (1995). Summary of low speed airfoil data (Vol. 1). SoarTech.

Shyy, W., Berg, M., Ljungqvist, D., 1999. Flapping and flexible wings for biological and micro air vehicles. Progress in Aerospace Science 35 (5), 455-505.

Taylor, G. I. "The Spectrum of Turbulence". 164 Proceedings of the Royal Society of London. Series A Mathematical and Physical Sciences. 1938

TR-AVT-202. "Extensions of Fundamental Flow Physics to Practical MAV Aerodynamics". AVT-202 Final Report.

Tropea C., Yarin A., and Foss J. Handbook of Experimental Fluid Mechanics, Springer Handbook

Ingen, J. L. van. "The $\mathrm{e}^{\mathrm{N}}$ method for transition prediction. Historical review of work at TU Delft" $38^{\text {th }}$ Fluid Dynamics Conference and Exhibit. Seattle, Washington. AIAA 2008

Ingen, J. L. van, "A Suggested Semi-empirical Method for the Calculation of the Boundary Layer Transition Region.” Report, VTH-74, 1956

Wagner, H., "Über die Entstehung des dynamischen Auftriebes von Tragflügeln". Zeitschrift fürangewandte Mathematik und Mechanik 5(1925) pp. 17-35.

Weis-Fogh T. "Quick estimates of flight fitness in hovering animals, including novel mechanism for lift production”. J Exp. Biol. 1973; 59:169-230. 